NIST Technical Note 1951

\title{
Industrial Wireless Systems Radio Propagation Measurements
}

\author{
Richard Candell \\ Kate A. Remley \\ Jeanne T. Quimby \\ David Novotny \\ Alexandra Curtin \\ Peter B. Papazian \\ Mohamed Kashef \\ Joseph Diener
}

This publication is available free of charge from:

https://doi.org/10.6028/NIST.TN.1951

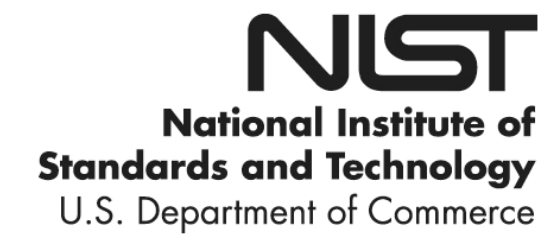


NIST Technical Note 1951

\title{
Industrial Wireless Systems Radio Propagation Measurements
}

\author{
Richard Candell \\ Intelligent Systems Division \\ Engineering Laboratory
}

Kate A. Remley

Jeanne T. Quimby

Peter B. Papazian

David Novotny

Joseph Diener

Alexandra Curtin

RF Technology Division Communications Technology Laboratory

Mohamed Kashef Advanced Network Technology Division Information Technology Laboratory

This publication is available free of charge from: https://doi.org/10.6028/NIST.TN.1951

January 2017

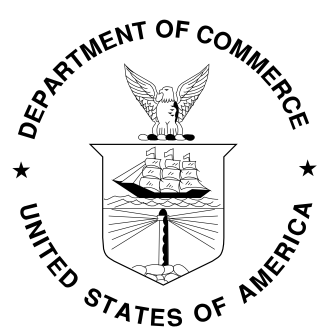

U.S. Department of Commerce Penny Pritzker, Secretary

National Institute of Standards and Technology Kent Rochford, Acting NIST Director and Under Secretary of Commerce for Standards and Technology 
Certain commercial entities, equipment, or materials may be identified in this document in order to describe an experimental procedure or concept adequately. Such identification is not intended to imply recommendation or endorsement by the National Institute of Standards and Technology, nor is it intended to imply that the entities, materials, or equipment are necessarily the best available for the purpose.

National Institute of Standards and Technology Technical Note 1951 Natl. Inst. Stand. Technol. Tech. Note 1951, 125 pages (January 2017) CODEN: NTNOEF

\section{This publication is available free of charge from: https://doi.org/10.6028/NIST.TN.1951}




\section{Contents}

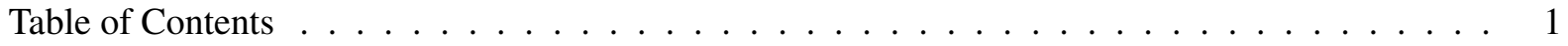

1 Introduction $\quad 1$

2 Site Descriptions $\quad 2$

2.1 Automotive Factory . . . . . . . . . . . . . . . . . . . . . 2

2.2 Steam Generation Plant . . . . . . . . . . . . . . . . . . . . . . 2

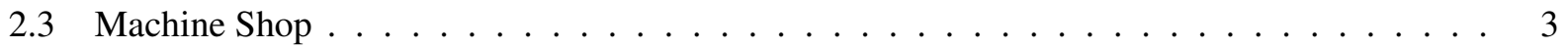

2.4 NIST Open Area Test Site $\ldots \ldots \ldots \ldots \ldots$

3 Measurement and Analysis Procedure $\quad 3$

3.1 Measurement Instrumentation $\ldots \ldots \ldots \ldots \ldots \ldots$

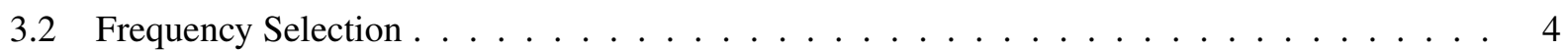

3.3 Measurement Acquisition . . . . . . . . . . . . . . . . . . . . 4

3.4 Storage and Post-processing $\ldots \ldots \ldots \ldots \ldots \ldots \ldots$

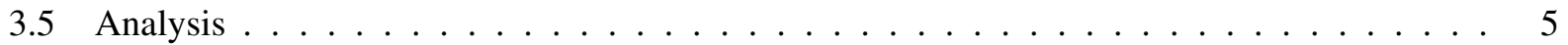

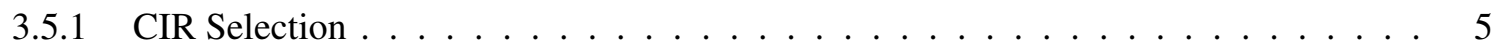

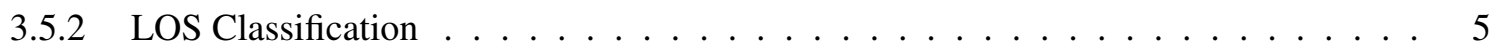

3.5 .3 Channel Gain . . . . . . . . . . . . . . . . . . . . . 6

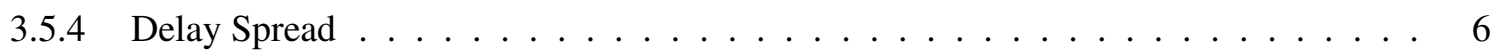

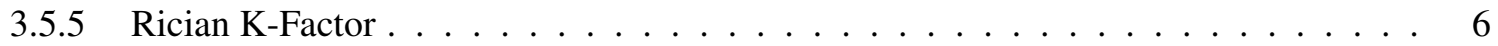

4 Summary of Results $\quad 6$

4.1 Removal of Outliers . . . . . . . . . . . . . . . . . . . . . . 6

4.2 Channel Gain . . . . . . . . . . . . . . . . . . . . . . . . 7

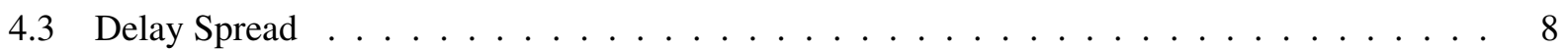

4.4 Rician K-factor . . . . . . . . . . . . . . . . . . . . . . 10

5 Conclusion $\quad 11$

$\begin{array}{lr}\text { A Appendix } & 13\end{array}$

A.1 Project Artifacts . . . . . . . . . . . . . . . . . . . 13

A.2 Site Layouts . . . . . . . . . . . . . . . . . . . . . . . 14

A.2.1 Automotive Assembly Factory . . . . . . . . . . . . . . . . . . . . 14

A.2.2 NIST Boulder Steam Plant . . . . . . . . . . . . . . . . . . . 15

A.2.3 NIST Machine Shop . . . . . . . . . . . . . . . . . . . 16

A.2.4 NIST Open Area Test Site . . . . . . . . . . . . . . . . . . . . . . . . . . . . . . . .

A.3 Run Summaries . . . . . . . . . . . . . . . . . . . . . . . . 18

A.4 Detailed Results . . . . . . . . . . . . . . . . . . . . . 20

A.4.1 AAPlantD1 2GHz TX1 hpol run4 pp.mat . . . . . . . . . . . . . . . 21

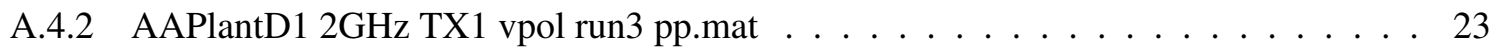

A.4.3 AAPlantD2 2GHz TX1 vpol internal runF pp.mat . . . . . . . . . . . . . 25

A.4.4 AAPlantD2 2GHz TX1 hpol internal runE pp.mat . . . . . . . . . . . . . . . 27

A.4.5 AAPlantD2 2GHz TX1 vpol internal runF pp.mat . . . . . . . . . . . . . . . . 29

A.4.6 AAPlantD2 5GHz TX1 hpol internal runB pp.mat . . . . . . . . . . . . 31

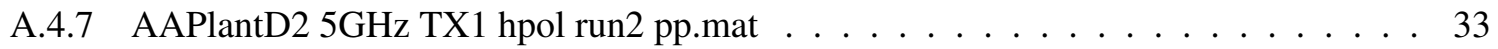

A.4.8 AAPlantD2 5GHz TX1 vpol internal runA pp.mat $\ldots \ldots \ldots \ldots$ 
A.4.9 AAPlantD2 5GHz TX1 vpol run1 pp.mat . . . . . . . . . . . . . . 37

A.4.10 AAPlantD3 2GHz TX2b hpol internal run36 pp.mat . . . . . . . . . . . . . 39

A.4.11 AAPlantD3 2GHz TX2b vpol internal run33 pp.mat . . . . . . . . . . . . . 41

A.4.12 AAPlantD3 2GHz TX2b vpol run32 pp.mat . . . . . . . . . . . . . . . . 43

A.4.13 AAPlantD3 2GHz Tx2b hpol run34 pp.mat . . . . . . . . . . . . . . . . . . . . . . . . . 45

A.4.14 AAPlantD3 5GHz TX2b hpol internal run39 pp.mat . . . . . . . . . . . . . 47

A.4.15 AAPlantD3 5GHz TX2b hpol run38 pp.mat . . . . . . . . . . . . . . . . . . 49

A.4.16 AAPlantD3 5GHz TX2b vpol internal run40 pp.mat . . . . . . . . . . . . 51

A.4.17 AAPlantD3 5GHz TX2b vpol run37 pp.mat . . . . . . . . . . . . . . . 53

A.4.18 GBurgD2 2GHz TX1 crossPol run3 pp.mat . . . . . . . . . . . . . . . . . 55

A.4.19 GBurgD2 2GHz TX1 longPol run2 pp.mat . . . . . . . . . . . . . . . 57

A.4.20 GBurgD2 2GHz TX1 vpol run1again pp.mat . . . . . . . . . . . . . . . . 59

A.4.21 GBurgD2 5GHz TX1 crossPol run3 pp.mat . . . . . . . . . . . . . . . . 61

A.4.22 GBurgD2 5GHz TX1 longPol run4 pp.mat . . . . . . . . . . . . . . . 63

A.4.23 GBurgD2 5GHz TX1 vpol run2 pp.mat . . . . . . . . . . . . . . . 65

A.4.24 GBurgD2 5GHz TX2high crossPol pp.mat . . . . . . . . . . . . . . 67

A.4.25 GBurgD2 5GHz TX2high longPol pp.mat . . . . . . . . . . . . . . . . . 69

A.4.26 GBurgD2 5GHz TX2low crossPol pp.mat . . . . . . . . . . . . . . 71

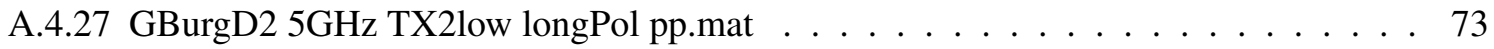

A.4.28 GBurgD2 5GHz TX2low vpol run1 pp.mat . . . . . . . . . . . . 75

A.4.29 GBurgD3 2GHz TX2high crossPol run2 pp.mat . . . . . . . . . . . . . . . . 77

A.4.30 GBurgD3 2GHz TX2high longPol run3 pp.mat . . . . . . . . . . . . . . . . 79

A.4.31 GBurgD3 2GHz TX2low crossPol run2 pp.mat . . . . . . . . . . . . . 81

A.4.32 GBurgD3 2GHz TX2low longPol run3 pp.mat . . . . . . . . . . . . . 83

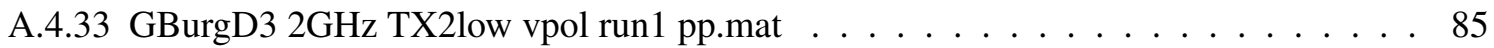

A.4.34 GburgD2 5GHz TX2high vpol pp.mat . . . . . . . . . . . . . . 87

A.4.35 GburgD3 2GHz TX2high vpol run1 pp.mat . . . . . . . . . . . . . . . . . 89

A.4.36 Oats $2 \mathrm{G}$ vpol 3115 run40 pp.mat . . . . . . . . . . . . . . . . . 91

A.4.37 Oats $2 \mathrm{G}$ vpol run39 pp.mat . . . . . . . . . . . . . . . . 93

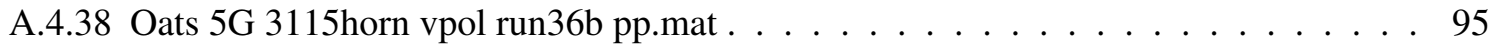

A.4.39 Tx2 2G Boiler 3115Vpol run1 pp.mat . . . . . . . . . . . . . . . . . 97

A.4.40 Tx2 2G Boiler 3115Vpol run2 pp.mat . . . . . . . . . . . . . . . . . . 99

A.4.41 Tx2 2G Boiler Vpol run2 pp.mat . . . . . . . . . . . . . . . . . . 101

A.4.42 Tx2 2G Boiler Vpol run3 pp.mat . . . . . . . . . . . . . . . . . . . . 103

A.4.43 Tx2 2G Boiler Vpol run4 pp.mat . . . . . . . . . . . . . . . 105

A.4.44 Tx2 5G Boiler 3115Vpol run1 pp.mat . . . . . . . . . . . . . 107

A.4.45 Tx2 5G Boiler 3115Xpol run1 pp.mat . . . . . . . . . . . . . . . . 109

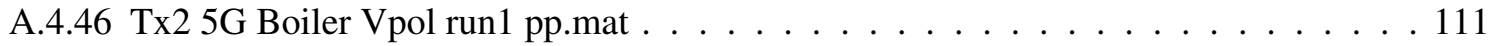

A.4.47 Tx2 5G Boiler Xpol run1 pp.mat . . . . . . . . . . . . . . . . . . 113

A.4.48 Tx2 5G Boiler 3115Vpol run1 pp.mat . . . . . . . . . . . . . . . . 115

A.4.49 Tx3 2G Boiler 3115Vpol run2 pp.mat . . . . . . . . . . . . . 117

A.4.50 Tx3 5G 3115Xpol run1 pp.mat . . . . . . . . . . . . . . . . . . . . . 119

A.4.51 Tx3 5G Boiler Vpol run1 pp.mat . . . . . . . . . . . . . . . . 121

A.4.52 Tx3 5G Boiler Xpol run1 pp.mat . . . . . . . . . . . . . . . . . . 123 


\begin{abstract}
Radio frequency (RF) propagation measurements were conducted at three facilities representing a crosssection of different classes of industrial environments. Selected sites included a multi-acre transmission assembly factory typical of the automotive industry; a small-sized machine shop primarily engaged in metalworking located on the NIST campus in Gaithersburg; and a steam generation plant located on the NIST campus in Boulder. A spread spectrum correlation sounder was used to take the measurements at a continuum of points throughout the facility by fixing the transmitter and moving the receiver at a constant rate throughout each facility. We analyzed the data collected from the RF propagation measurements of each site. Analysis is based on channel impulse response (CIR) measurements collected during the measurement using equipment developed by the National Institute of Standards and Technology. The results of our analysis include a tabulated summary and detailed exploration of various industry accepted channel metrics such as path loss, delay spread, and $\mathrm{K}$ factor. Our interpretation of the measurements contributes to an improved understanding of radio frequency propagation in factories and an additional perspective on deploying wireless communication devices within factories. The raw measurement data and analysis software are made freely available for public download.
\end{abstract}




\section{Introduction}

Industrial control systems are increasingly employing communications technologies to facilitate data sharing and analysis in integrated control operations. Within industrial control systems, wireless technologies are becoming increasingly prevalent as an enabling technology providing continuous awareness and control of the factory process. Wireless communication is often cited as a key enabling technology of the advanced manufacturing processes by eliminating the limitations and costs associated with conduit and cables but not without challenges $[1,2]$. Wireless communication helps to alleviate economic cost and agility constraints by being low-cost and mobile [3]. However, industrial wireless environments can be harsh as the surroundings are typically highly reflective, thereby creating significant multi-path effects and electromagnetic resonance. Industrial control systems are often designed to be intolerant of communication faults and often require very high transmission reliability. Error correction schemes are plausible to counteract communications problems; however, a rigorous understanding of radio frequency (RF) propagation within the factory is essential to achieving the communications requirements of an industrial control system.

New wireless protocols have been developed over the last decade such as ISA100.11a [4] and WirelessHART [5] which address the need for remote sensing and provide some mechanisms of actuation. Both of these protocols are based on the IEEE 802.15.4 standard. Other wireless systems employ the IEEE 802.11 standard and provide a mechanism for supplanting input-output (IO) wires with a wireless link. These types of links are used for both sensing and control. They often employ proprietary application layer protocols. IEEE 802.11 links appear to existing control systems as wires and theoretically require little or no modification of the underlying control algorithm. This offers enormous deployment flexibility as existing sensors, actuators, and process control devices need not be modified when a wired link fails. However, factory engineers hesitate to adopt wireless options due to a perceived lack of reliability, determinism, and security of wireless solutions compared to wired equivalents.

Our work addresses those concerns through a multi-phased approach, including active channel characterization measurements which is the focus of this report, simulation, and cyberphysical systems experimentation in the laboratory [6]. By studying RF propagation of radio waves in the bands of interest for industrial application, namely the $2.4 \mathrm{GHz}$ and $5 \mathrm{GHz}$ bands, using a measurement science approach, we can better understand and address the challenges of deploying wireless using existing standards. We can also use this understanding to develop better wireless solutions for advanced manufacturing systems that require ultra-low latency and high reliability. This report explores the measurements collected in May 2015 of a transmission assembly factory in Detroit and two other factory sites. Other factory radio propagation measures conducted by NIST of an automotive factory similar to the automotive factory examined by this work is described in [7].

Finally, we make our measurements and associated analysis openly available for download [8]. Our computer code used for our analysis is also freely available [9]. 


\section{Site Descriptions}

\subsection{Automotive Factory}

The automotive factory chosen for this study was a multi-acre indoor transmission assembly factory with large tracts of open areas similar to those described in [7]. The factory had floor dimensions of over $400 \mathrm{~m}$

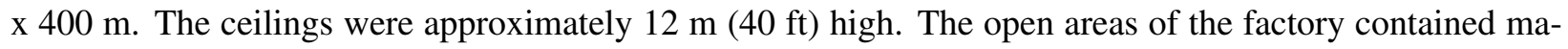
chining areas, inspection machines, assembly work cells, and stacked storage areas. Enclosed spaces were used to store factory inventory of small parts and tools. Storage areas were not included in the study. Two distinct routes were chosen for our measurements and are described in the following sections. To protect the intellectual property of the automotive factory owners, we do not show machines on the diagram; however, it was markedly dense with tall machines constructed of metal. Some interior concrete wall construction was present.

\section{Measurement Path: Outer Loop}

The first route, the outer loop Fig. A.2.1, was chosen to represent paths taken by autonomous ground vehicles (AGVs) and other mobile platforms deployed throughout the factory for transportation of parts and inventory. The outer loop also represented the path of future robotic platforms anticipated by the factory owner. Important to the outer loop route is the distance of the receive antenna from surrounding radio wave reflectors such as machine housings.

\section{Measurement Path: Inner Loop}

The second route, the inner loop Fig. A.2.2, was chosen to represent those areas of the factory exhibiting reflective metals, machine canyons, and overhead robot gantry systems. An important characteristic of the inner loop is the lack of a line-of-sight (LOS) path to the transmitter. Stationary measurements were conducted at points I2 and I6.1. While it is unlikely that wireless devices would be deployed directly at these locations, I2 and I6.1 were selected to represent worst case scenario locations within the factory. The inner loop measurements are also referred to throughout the data as internal. The inner loop measurements include a two minute pause within two metal canyon regions. These are locatable using a descriptive metadata associated with the measurements (refer to Section A.1 for project artifacts).

\subsection{Steam Generation Plant}

The steam generation plant was chosen as a class of indoor industrial site with large machinery and overhead obstructions. The steam plant factory layout is shown in A.2.3. A picture of the facility is provided and gives the reader a better understanding of the physical environment which includes several boilers and overhead piping. The outer dimensions of the building are approximately $50 \mathrm{~m}$ x $80 \mathrm{~m}(164 \mathrm{ft} \times 262 \mathrm{ft})$, with a ceiling height of approximately $7.6 \mathrm{~m}(25 \mathrm{ft})$. The building is divided into two sections, with boilers on one side and with refrigeration equipment on the other. A cinder block wall divides the two sides. Two large openings approximately $3 \mathrm{~m}(10 \mathrm{ft})$ wide join the sides at each end. The boiler section, where our measurements were conducted, was approximately $20 \mathrm{~m}$ x $80 \mathrm{~m}$ (66 ft x $262 \mathrm{ft}$ ). The walls are made of metal, concrete, and glass. Two transmit antenna locations were chosen to characterize the facility, TX2 and TX3. The TX2 position was situated on the ground floor in a location that would typically be chosen for a wireless access point. The TX3 position was situated on the second level overlooking the steam plant with large pipes and conduits that could serve as waveguides and reflectors. 


\subsection{Machine Shop}

The machine shop was selected as our first facility for our measurements. The machine shop is considered

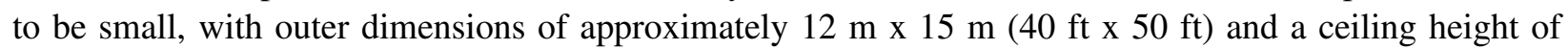

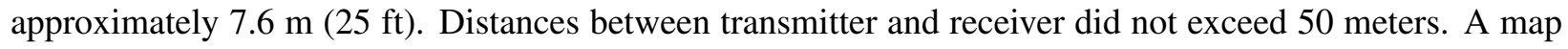
of the shop floor is shown in A.2.5.

\subsection{NIST Open Area Test Site}

The NIST Open Area test site (OATS) is an open range facility that is used for calibration and reference measurements of radio wave propagation. The OATS facility is located at the NIST campus in Boulder, Colorado. It is situated in an elevated open space with minimal interference from unexpected emitters and has controllable multi-path components. The instrumentation used for factory site measurements was compared to known ray-tracing models using OATS. Measurement data from the OATS facility are included in this report.

\section{Measurement and Analysis Procedure}

The following sections describe the process by which our analysis was performed. The descriptions are intended as a high-level summary of the process; however, a detailed examination is possible by inspecting the computer code used for the analysis as identified in Section 1.

\subsection{Measurement Instrumentation}

The NIST channel sounder system is a positive-negative $(\mathrm{PN})$ sequence correlation system with an operating frequency range of $65 \mathrm{MHz}$ to $6 \mathrm{GHz}$ [10]. The instrument supports a $250 \mathrm{MHz}$ instantaneous bandwidth. It consists of a single transmitter with power amplifier and a receiver coordinated with two synchronized rubidium clocks. The transmitter repetitively transmits a PN sequence of digital symbols modulated with a Binary Phased-Shift Keying (BPSK) signal and up-converts to the RF carrier frequency. After traveling through a power amplifier, the signal traverses the factory and is detected by the channel sounder receiver as shown in the Fig. 3.1.1. The receiver down-converts, digitizes, and stores the received signal locally. Removal of the hardware impairments occur using an equalization step performed during post-processing. Equalization is performed using the back-to-back measurements taken prior to measurement. 


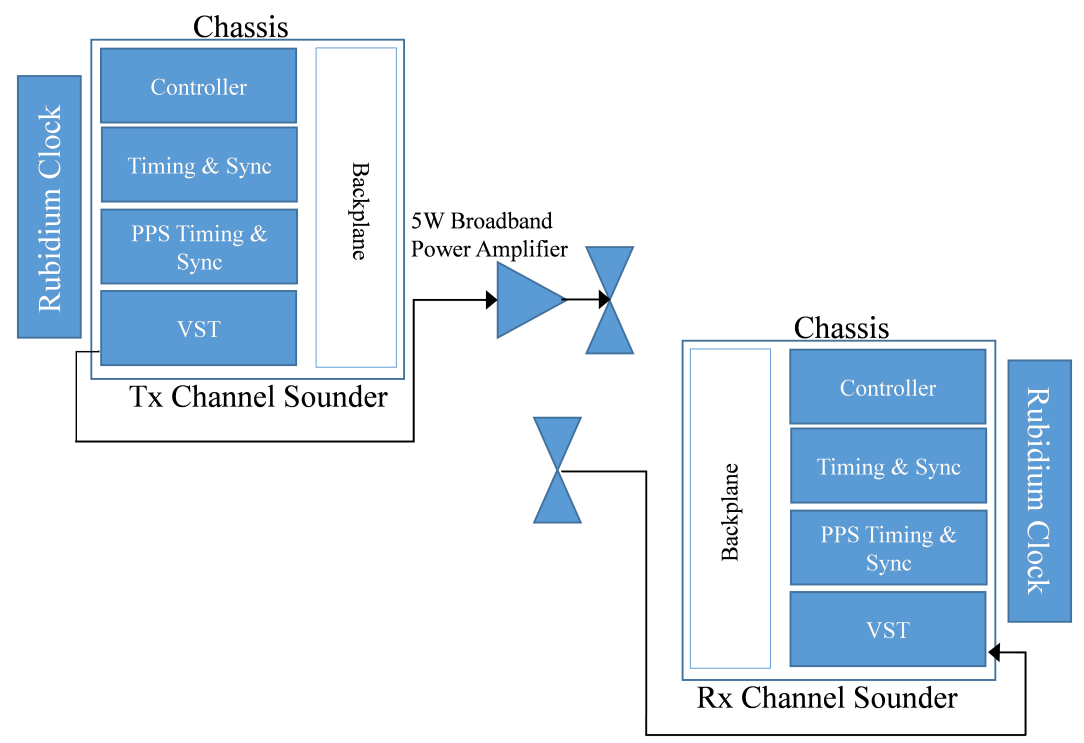

Figure 3.1.1: The NIST channel sounder apparatus is used for collecting synchronized radio propagation measurements. The instrument provides precision digitization and supports a large RF tuning range with a $250 \mathrm{MHz}$ bandwidth that can accommodate most bands of interest for industrial applications.

\subsection{Frequency Selection}

Transmit center frequencies $2.25 \mathrm{GHz}$ and $5.4 \mathrm{GHz}$ were chosen for two reasons. First, because they are licensed U.S. government bands, we could perform the measurements without generating interference to or encountering it from local wireless devices. Second, because the selected government bands are near to the unlicensed industrial, scientific, and medical (ISM) radio bands used within manufacturing facilities, our measurements represent what would be experienced by equipment in the ISM bands used in a typical manufacturing facility.

\subsection{Measurement Acquisition}

Measurements of the outer loop were conducted by moving the receiver cart along the planned route at a constant speed. The routes started and ended near to the transmitter, as synchronization was required prior to each measurement run. At some sites, the cart may have been paused for momentary obstacles such as machine traffic and human operated vehicles. The pause was noted in our technical notes, and cart motion was resumed once the obstacle passed. Each route followed a predetermined path throughout the facility, passing machines, circumnavigating storage areas, running behind walls, finally returning to the starting position. By taking different routes, it was possible to capture measurements representing many classes of regions within the sites except those that were prohibited for safety reasons at the automotive facility. At the end of each route, a back-to-back measurement was taken to determine the amount of change in the clock synchronization and RF characteristics of the measurement hardware.

\subsection{Storage and Post-processing}

Raw measurement data for each run was stored as complex baseband samples in National Instruments test data exchange stream (TDMS) file format. Runs were divided into acquisitions and then records. Each 
run resulted in multiple acquisitions, and each acquisition contained multiple records. Acquisitions were associated with way-points, and records were associated with actual measurements between the way-points. Each record contained the complex value noise signal requiring further processing using cross-correlation to produce the complex channel impulse response (CIR) for each record. This process is described in [11]. For each measurement run, channel impulse responses are stored in MATLAB (MAT) file format with associated Cartesian coordinates and time information. Meta-data describing the parameters of each run are included in each file making analysis convenient. It is important to note that the files are large, and we advise that researchers making use of these measurements have at least $16 \mathrm{~GB}$ of RAM available to load the files.

\subsection{Analysis}

Analysis of the measurement data was conducted in accordance with ITU Recommendation IT-P14075 [12]. The process for calculation of the channel metrics involves significant computing resources to manipulate the data into formats for data analysis. That process is not elaborated here as the code for our analysis is made publicly available and is referenced in A.1. The underlying equations used to produce channel metrics for each run underlying the tabulated metrics and per-run analyses are given here.

\subsubsection{CIR Selection}

We start the CIR processing for every record of the measured data by keeping the significant samples. The sample selection process is based on passing a threshold criteria which requires that we retain samples 10 $\mathrm{dB}$ above the computed noise floor and within $30 \mathrm{~dB}$ from the CIR peak power. We begin by calculating the noise floor which is denoted by $N$ and is calculated as follows

$$
N=\frac{1}{T} \sum_{t=0}^{T}|x(t)|^{2} .
$$

We then obtain a modified CIR which is denoted by $x_{\mathrm{m}}$ by retaining any sample which satisfies the threshold conditions. The peak of $x(t)$ is denoted by $\hat{x}(t)$. As a result, the modified CIR is calculated as follows

$$
x_{\mathrm{m}}= \begin{cases}x(t), & |x(t)|^{2} \geq 10 N \text { and }|x(t)|^{2} \geq 10^{-3}|\hat{x}(t)|^{2}, \\ 0, & \text { otherwise }\end{cases}
$$

We then estimate various corresponding parameters to the measured CIRs. We start by classifying the CIRs according to channel visibility, either line-of-sight (LOS) channel or non-line-of-sight(NLOS). Visibility is computed numerically from the CIR. We then explain our techniques for evaluating various CIR characteristics such as path loss, delay spread, and K-factor. Average channel response requires spatial correlation of CIR records and is outside of the scope of this report.

\subsubsection{LOS Classification}

We build this classification based on the value of the first peak of the CIR. If the magnitude of the first peak is also the maximum of all peaks, then the CIR is classified to be LOS; otherwise; it is classified as NLOS. We define an indicator $I_{\text {LOS }}$ which equals 1 for LOS CIRs and - 1 for NLOS. We also denote the value of the first peak of $x(t)$ by $\hat{x}_{1}(t)$. The LOS indicator is calculated as follows

$$
I_{\mathrm{LOS}}= \begin{cases}1, & \max (|\hat{x}(t)|)=\left|\hat{x}_{1}(t)\right| \\ -1, & \text { otherwise }\end{cases}
$$




\subsubsection{Channel Gain}

At each point through the receiver path, we calculate the Euclidean distance between between the transmitter and receiver antennas. Then, we evaluate the path loss at each distance. The channel path gain is denoted by $G$ and is calculated in $\mathrm{dB}$ as follows

$$
G(\mathrm{~dB})=10 \log \sum_{t=0}^{T}\left|x_{\mathrm{m}}\right|^{2}-G_{\mathrm{tx}}(\mathrm{dB})-G_{\mathrm{rx}}(\mathrm{dB}),
$$

where $G_{\mathrm{tx}}$ and $G_{\mathrm{rx}}$ are the transmitter and receiver gains in $\mathrm{dB}$, respectively. Often, we consider path loss rather than gain. Path loss is merely the negative of path gain.

\subsubsection{Delay Spread}

The delay spread calculation is an important estimate of measuring the diffuseness of a signal. It can also be considered as a measure of the multi-path richness. Delay spread is calculated in accordance with ITU-R P.1407-5 [12].

We first calculate the mean delay which is denoted by $\bar{\tau}$ as follows

$$
\bar{\tau}=\frac{\sum_{t=0}^{T} t\left|x_{\mathrm{m}}(t)\right|^{2}}{\sum_{t=0}^{T}\left|x_{\mathrm{m}}(t)\right|^{2}} .
$$

The RMS delay spread is the second moment of the CIR power delay which describes the weighted multi-path variance from the mean delay and is evaluated as follows

$$
\tau_{\mathrm{rms}}=\sqrt{\frac{\sum_{t=0}^{T}(t-\bar{\tau})^{2}\left|x_{\mathrm{m}}(t)\right|^{2}}{\sum_{t=0}^{T}\left|x_{\mathrm{m}}(t)\right|^{2}}} .
$$

\subsubsection{Rician K-Factor}

Rician K-factor ("K") is generally defined to be the ratio between the power of the LOS component to the mean power of diffuse components. As we have defined the first peak to be $\hat{x}_{1}(t)$, we denote the $i$ th peak by $\hat{x}_{i}(t)$ where the last peak index is denoted by $I$. As a result, the $\mathrm{K}$-factor of a CIR is calculated as follows

$$
K(\mathrm{~dB})=10 \log \frac{\hat{x}_{1}^{2}(t)}{\frac{1}{I-1} \sum_{i=2}^{I} \hat{x}_{i}^{2}(t)} .
$$

\section{Summary of Results}

The following sections provide a summary exploration of the results of our analysis. A deeper study requires an examination of the detailed analysis presented in Section A.4 or more investigation using the open measurement data. Within each section, we also present results from the open area test site (OATS) for validation of our methods and comparison to the channel characteristics of the industrial environment.

\subsection{Removal of Outliers}

Outliers present in this data provide little value in predicting communications systems behavior because of how seldom they occur in the datasets. By removing statistical outliers, we provide a more meaningful estimate of delay spread and K-factor statistics by removing measurements that would otherwise distort 
mean and variance calculations of those measurements. Delay spread and K-factor estimates were, therefore, preprocessed to remove outliers before statistical metrics were calculated. An iterative Grubbs test was used to detect single sample outliers and remove them using code provided in [13].

\subsection{Channel Gain}

A summary of channel gain calculations is provided in Table 4.1. The table lists factory site, frequency, route, slope of the first-order least squares fit of the gain (using the second fit region), and the intercept. The intercept is defined as the theoretical gain at $d=0$ meters. Detailed plots of the gain curves are given in A.4. For each run, the gain plot is shown with a two-segment least-squares fit. The second segment slope is given as the path loss exponent, $\gamma_{2}$, where loss in $\mathrm{dB}$ is $L=10 \log _{10}\left(d^{\gamma}\right)+20 \log _{10}(f)-147.55$. A comparison of gain to OATS shows that the industrial environments tend to lose power more rapidly as a function of distance. The automotive factory in particular loses power presumably to the higher density of machines as compared to the machine shop and the larger dimensions of the facility.

Table 4.1: Channel Gain Summary for All Sites

\begin{tabular}{|c|c|c|c|c|}
\hline Site & $f(\mathbf{G H z})$ & Route & $\gamma_{2}$ & Intercept (dB) \\
\hline \hline oats & 2.2 & external & 1.8 & -40.4 \\
\hline oats & 5.4 & external & 1.9 & -47.2 \\
\hline aaplant & 2.2 & external & 4.3 & -6.2 \\
\hline aaplant & 2.2 & internal & 5.9 & 20.8 \\
\hline aaplant & 5.4 & external & 3.9 & -19.0 \\
\hline aaplant & 5.4 & internal & 5.8 & 15.4 \\
\hline shop & 2.2 & external & 1.9 & -42.3 \\
\hline shop & 5.4 & external & 1.6 & -55.1 \\
\hline steam & 2.2 & external & 2.7 & -31.0 \\
\hline steam & 5.4 & external & 2.8 & -40.5 \\
\hline
\end{tabular}

Gain curves for $2.245 \mathrm{GHz}$ and $5.4 \mathrm{GHz}$ are shown in Figures 4.2.1a and 4.2.1b. As is customary, we have approximated the gain curves using a two segment linear regression. The polynomial fit approximation and an estimate of the root mean squared error in predicting a future observations are shown in the legend. The polynomial fit and error estimations were approximated using a two-segment least squares fit in which the slopes, Y intercepts, and inflection points are varied to minimize the mean squared error. The model for the least squares fit is given as

$$
F(x)=(x<Q) *(a 1 * x+b 1)+(x>Q) *(a 2 * x+b 2)
$$

where $\mathrm{Q}$ is the inflection point.

Gain curves for each dataset provided in Section A.4 were calculated with inflection points of 30, 10, 5, and 5 meters for the automotive plant, machine shop, steam plant, and OATS facilities, respectively. Further error reduction is possible by selecting an improved path gain model. 


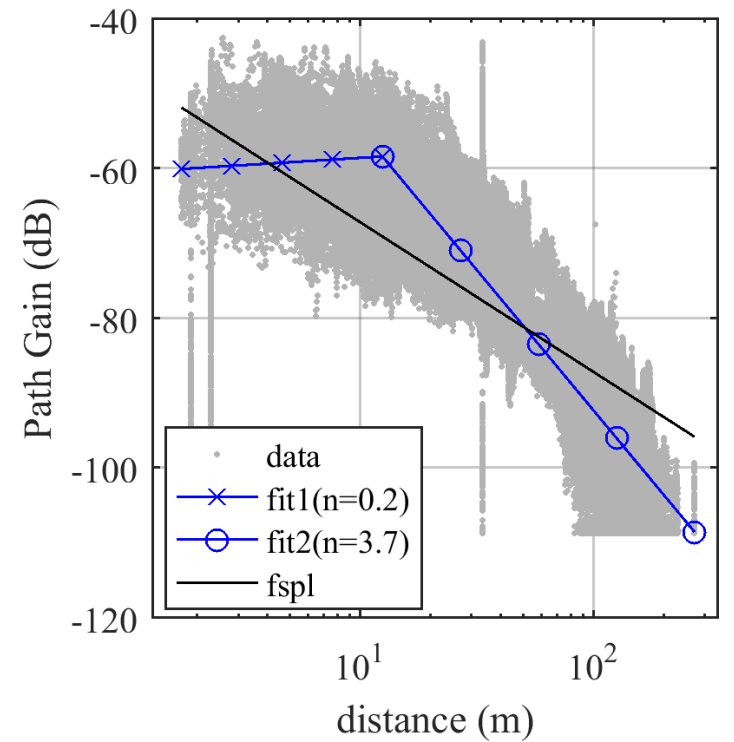

(a)

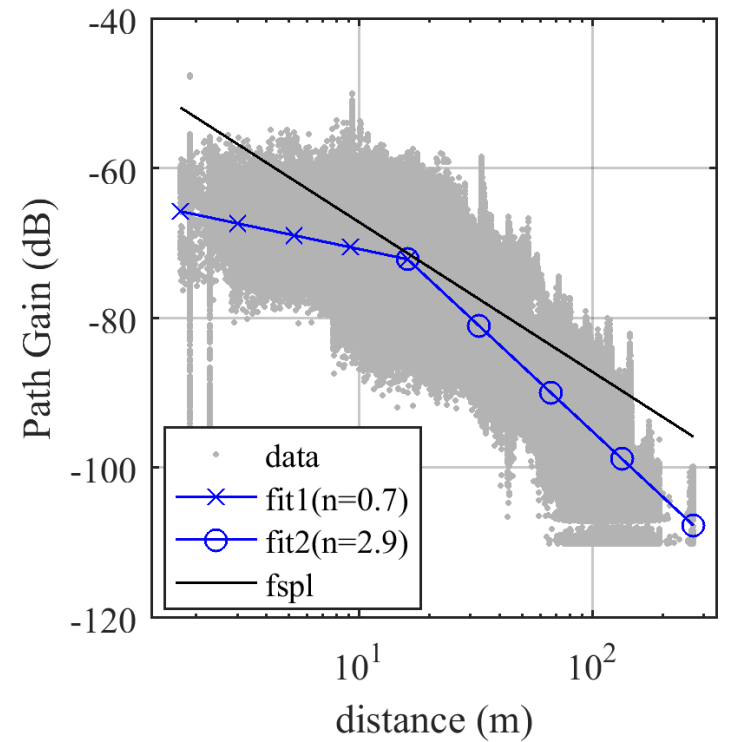

(b)

Figure 4.2.1: Gain data for measurements at (a) $2.245 \mathrm{GHz}$ and (b) $5.4 \mathrm{GHz}$.

\subsection{Delay Spread}

A summary of power delay and delay spread are given in Table 4.2. This section is useful for determining impact of multi-path to receiver performance. The impact of the power delay spread of a signal depends on the underlying transmitted symbol rate, modulation, and receiver architecture used by the communication system. Mean Delay, $\bar{\tau}$, is a first order estimate of the power-weighted tap delay of the impulse response. Delay spread, $\bar{S}$, provides a measure of the diffuseness of the impulse response. Delay spread is typically considered the more useful metric of channel behavior as it gives an indication of how much a signal is diffused over time. It is helpful in digital receiver design and the prediction of transmission reliability. The expected value, $\mathbf{E}(\cdot)$, denotes the mean.

An analysis of the table shows that the automotive plant has a high delay spread than the machine shop and steam plant. This may be due to the physical dimensions and layout of the automotive plant compared to the steam plant and the machine shop. As expected, the OATS facility has a very low delay spread as the multi-path is controlled. 
Table 4.2: Expected values for Power Delay Spread Summary for All Sites

\begin{tabular}{|c|c|c|c|c|c|}
\hline Site & $f(\mathbf{G H z})$ & Route & $\mathbf{E}(\min )$ & $\mathbf{E}($ mean $)$ & $\mathbf{E}(\max )$ \\
\hline \hline oats & 2.2 & external & 5.5 & 5.8 & 6.2 \\
\hline oats & 5.4 & external & 6.1 & 6.1 & 6.1 \\
\hline aaplant & 2.2 & external & 106.6 & 115.5 & 121.7 \\
\hline aaplant & 2.2 & internal & 111.9 & 131.8 & 141.1 \\
\hline aaplant & 5.4 & external & 138.6 & 162.1 & 179.1 \\
\hline aaplant & 5.4 & internal & 146.0 & 154.4 & 162.6 \\
\hline shop & 2.2 & external & 63.6 & 74.3 & 81.3 \\
\hline shop & 5.4 & external & 54.7 & 70.1 & 78.3 \\
\hline steam & 2.2 & external & 32.1 & 36.1 & 42.2 \\
\hline steam & 5.4 & external & 30.9 & 45.4 & 54.9 \\
\hline
\end{tabular}

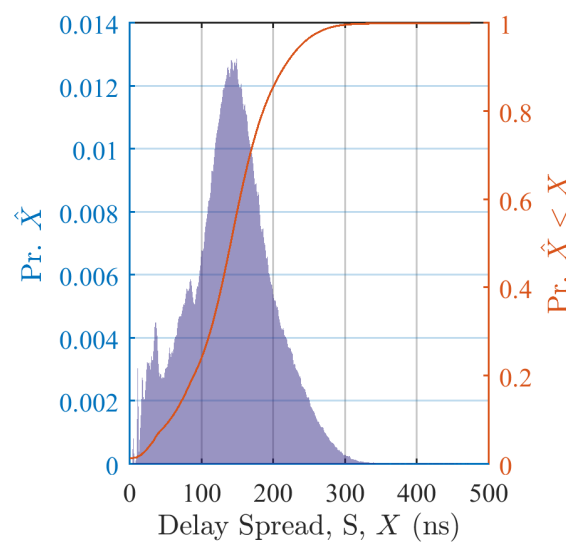

(a)

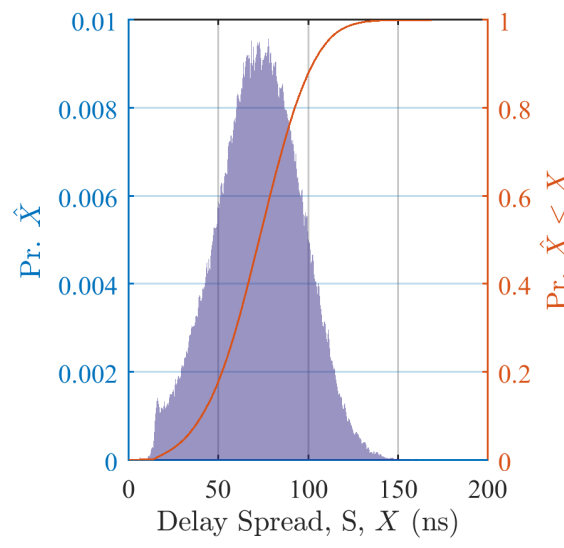

(c)

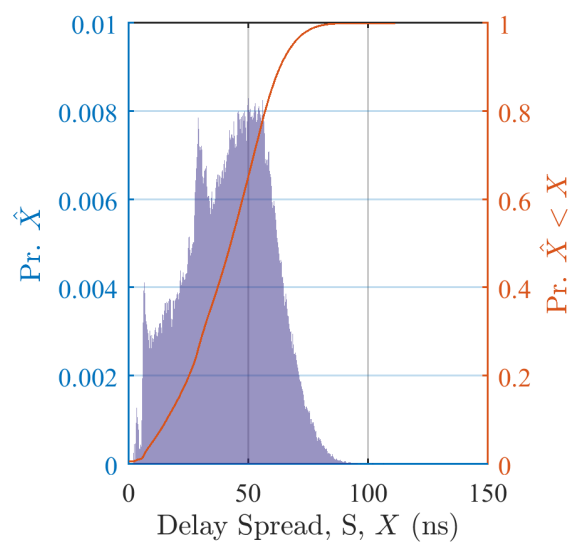

(b)

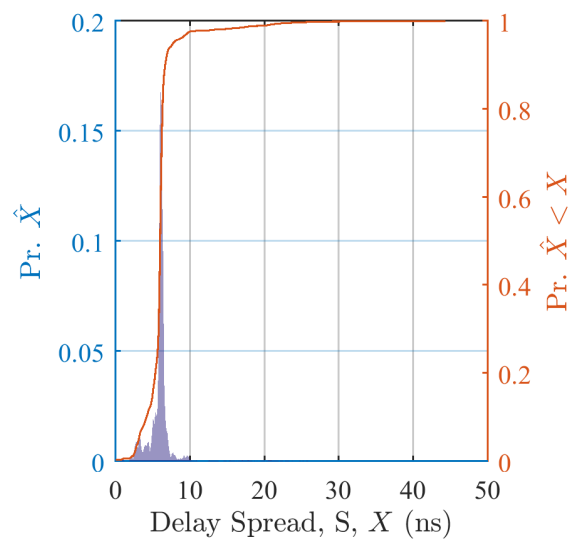

(d)

Figure 4.3.1: Delay spread estimates for (a) automotive factory, (b) steam plant, (c) machine shop, and (d) the OATS facility. Measurements shown include all frequencies. 


\subsection{Rician K-factor}

Rician K-factor is summarized in Table 4.3. Typical outdoor K-factors range between $6 \mathrm{~dB}$ and $30 \mathrm{~dB}$. A typical figure of merit for a satellite channel is $10 \mathrm{~dB}$ which indicates that the LOS component is 10 times more powerful than the sum of its multi-path components. The average K-factor for our facilities ranged from $-5 \mathrm{~dB}$ to $6 \mathrm{~dB}$. This indicates a strong multi-path channel if LOS and NLOS channels are considered.

It is important to note that $\mathrm{K}$-factor is sometimes calculated for channels with a known LOS status where the LOS status is assured through ray tracing or direct observation; however, this can be misleading as the definition of $\mathrm{K}$-factor allows for values of $\mathrm{K}$ that can approach $-\infty$. For industrial settings, a LOS situation is seldom the case especially for mobile platforms; therefore, in our analysis, $\mathrm{K}$ is calculated without regard for the presence of a LOS component. For the steam plant sites, measurements were sometimes taken with the transmitter situated above piping, demonstrating how factory environment can be quite harsh for wireless communications if propagation effects are not considered. Refer to the TX3 location detailed measurements of the steam plant site for examples of such a situation.

A comparison of the K-factor for the measured sites is shown in Fig. 4.4.1. The OATS facility measurements show a considerably higher $\mathrm{K}$ factor as expected due to the controlled multi-path environment. It is interesting to note that while the OATS measurements show a $\mathrm{K}$ factor range similar to the other sites, examination of OATS cumulative probability distributions in the detailed section reveals that $75 \%$ of the values are well above $15 \mathrm{~dB}$ and $50 \%$ of the are above $25 \mathrm{~dB}$. For the automotive factory, $75 \%$ of the $\mathrm{K}$ factors are above $4 \mathrm{~dB}$ and $50 \%$ of the Rician $\mathrm{K}$ factors are above $6 \mathrm{~dB}$. Less than $1 \%$ of the values rise above $20 \mathrm{~dB}$. Also of interest is the double lobe distribution associated with the steam plant measurements.

Table 4.3: Expected value for K-Factor Summary for All Sites

\begin{tabular}{|c|c|c|c|c|c|}
\hline Site & $f(\mathbf{G H z})$ & Route & $\mathbf{E}($ mean $)$ & $\mathbf{E}(\max )$ & $\mathbf{E}(\min )$ \\
\hline \hline oats & 2.2 & external & 21.9 & 30.0 & -9.8 \\
\hline oats & 5.4 & external & 12.2 & 30.0 & -17.3 \\
\hline aaplant & 2.2 & external & 2.6 & 23.5 & -21.2 \\
\hline aaplant & 2.2 & internal & 3.3 & 23.7 & -22.4 \\
\hline aaplant & 5.4 & external & 3.9 & 23.9 & -23.4 \\
\hline aaplant & 5.4 & internal & 3.2 & 24.7 & -23.1 \\
\hline shop & 2.2 & external & 5.8 & 25.4 & -24.4 \\
\hline shop & 5.4 & external & 5.5 & 24.3 & -22.9 \\
\hline steam & 2.2 & external & 5.9 & 24.9 & -23.8 \\
\hline steam & 5.4 & external & -4.3 & 22.3 & -25.2 \\
\hline
\end{tabular}




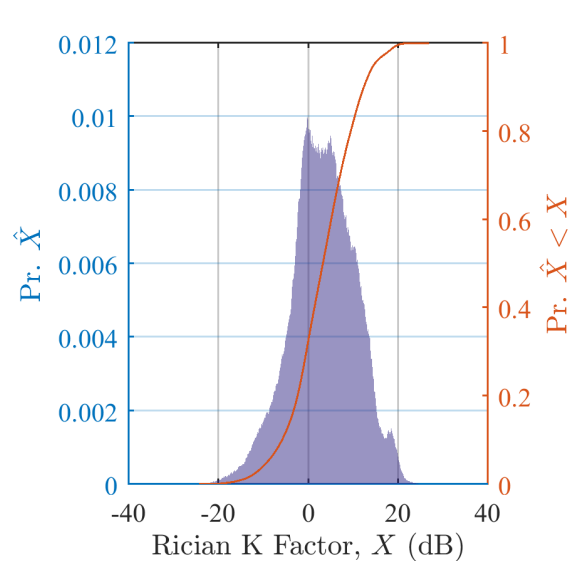

(a)

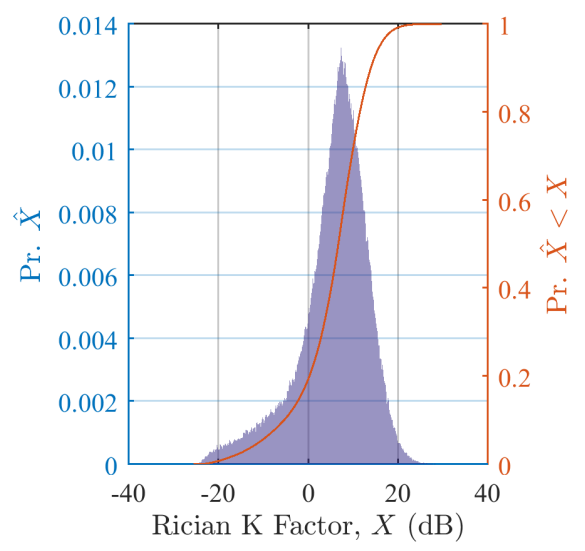

(c)

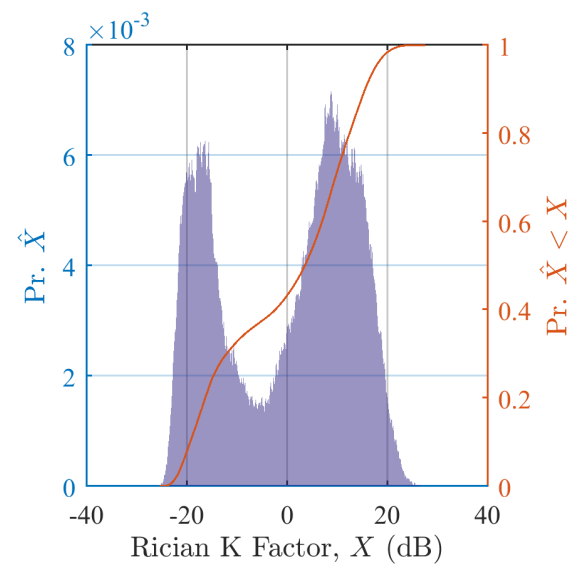

(b)

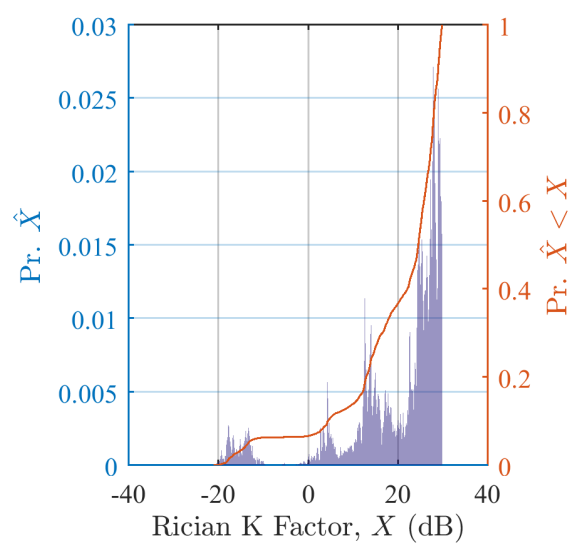

(d)

Figure 4.4.1: K factor for estimates for (a) automotive factory, (b) steam plant, (c) machine shop, and (d) the OATS facility. Measurements shown include all frequencies.

\section{Conclusion}

Understanding the channel characteristics of environments where wireless technologies will be deployed is the first step in designing and deploying reliable wireless networks. In particular, industrial environments such as those found in oil refineries and automotive facilities are considered harsh as compared to other environments due in part to the density of high reflective materials found at those locations. In this report, we present a numerical analysis of radio frequency propagation measurements taken at three different industrial facilities. We also collect similar data at the NIST OATS facility for validation and comparison.

Using our analysis we demonstrate how the physical environment impacts the impulse response (i.e., the channel) of wireless signals during flight. We present estimates of channel behavior using widely accepted methods such as those presented in [12]. Using this analysis and results in other works, it is possible to develop more rigorous solutions to difficult wireless communications problems such as selection of appropriate technologies and center frequencies, or placement of access points or nodes within an industrial network such that information reliability is maximized and impacts to the operation are minimized.

We should stress that although the industrial environment does exhibit strong multi-path characteristics as indicated by our delay spread and $\mathrm{K}$ factor estimates, this is not indication that all wireless solutions 
will fail. On the contrary, many other factors come into play when determining the impact of channel characteristics on industrial wireless system performance. We estimated average delay spreads below 500 ns which may be insignificant to a $256 \mathrm{kbps}$ protocol, but it may be severely limiting to a protocols with much higher transmitted symbol rates. Current industrial wireless technologies may be largely immune to multi-path effects, but we cannot make that assumption easily. Testing is required of existing technologies to determine the impacts of industrial wireless channel on factory operations.

Future low-latency, high-reliability systems will be required to take into account the characteristics of industrial wireless channels. We leave that analysis for later work; however, a list of factors would include the antenna technology employed, the underlying symbol rates, bit rate, receiver architecture and complexity, error control coding, network topologies, and protocols used.

Finally, our measurement data and computer code are made freely available for public download. The results presented here will be used to select appropriate wireless reliability models to conduct laboratory experiments and run simulations. 


\section{A Appendix}

\section{A.1 Project Artifacts}

Measurement Data Measurement data underlying this report is freely available and may be downloaded through our project data landing page [8].

Computer Code Computer code for this report is made freely available through the GitHub "IndustrialWirelessAnalysis" repository [9]. 


\section{A.2 Site Layouts}

\section{A.2.1 Automotive Assembly Factory}

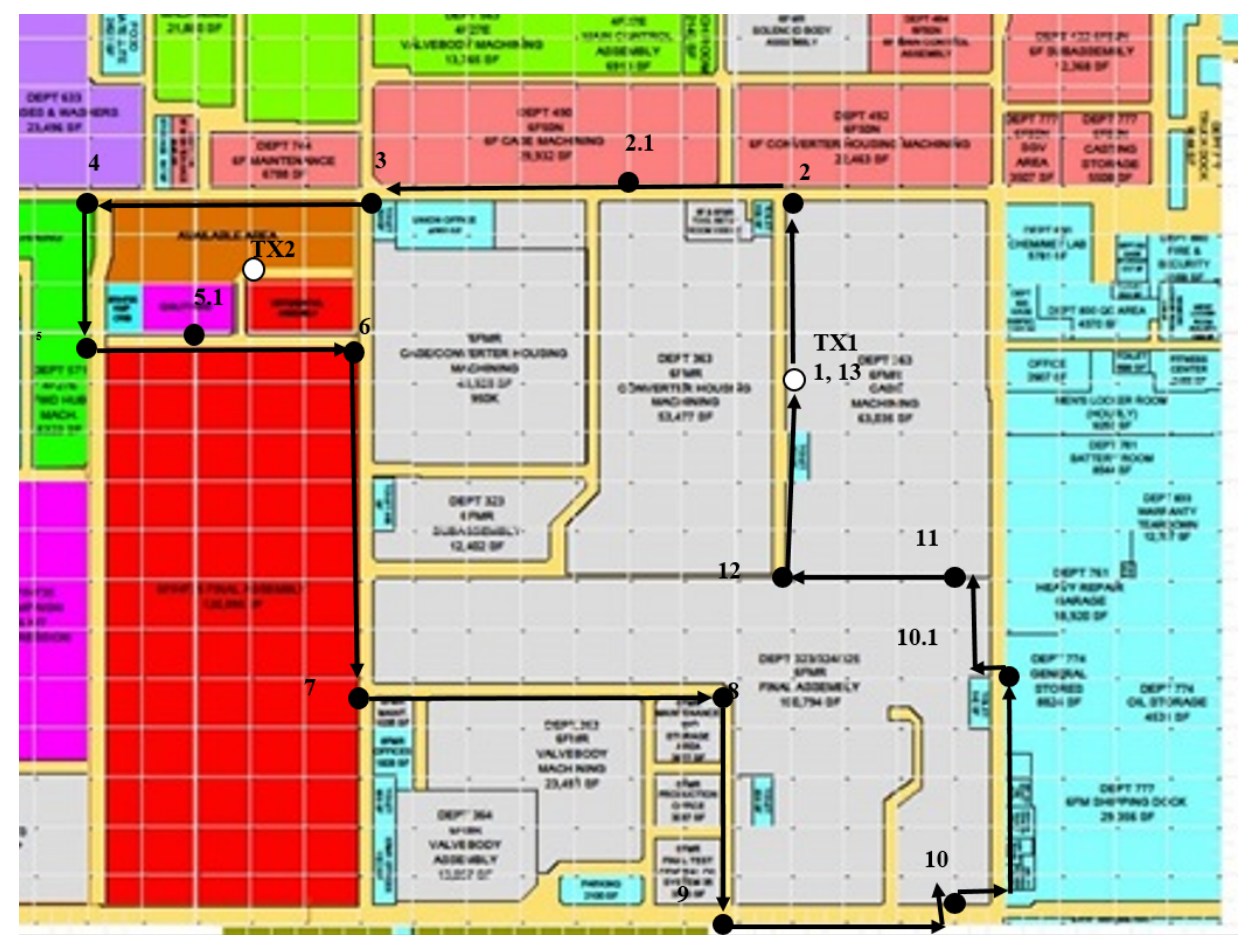

Figure A.2.1: Automotive Assembly Plant, Outer Loop Route. Each grid square represents 15.2 m x 15.2 m.

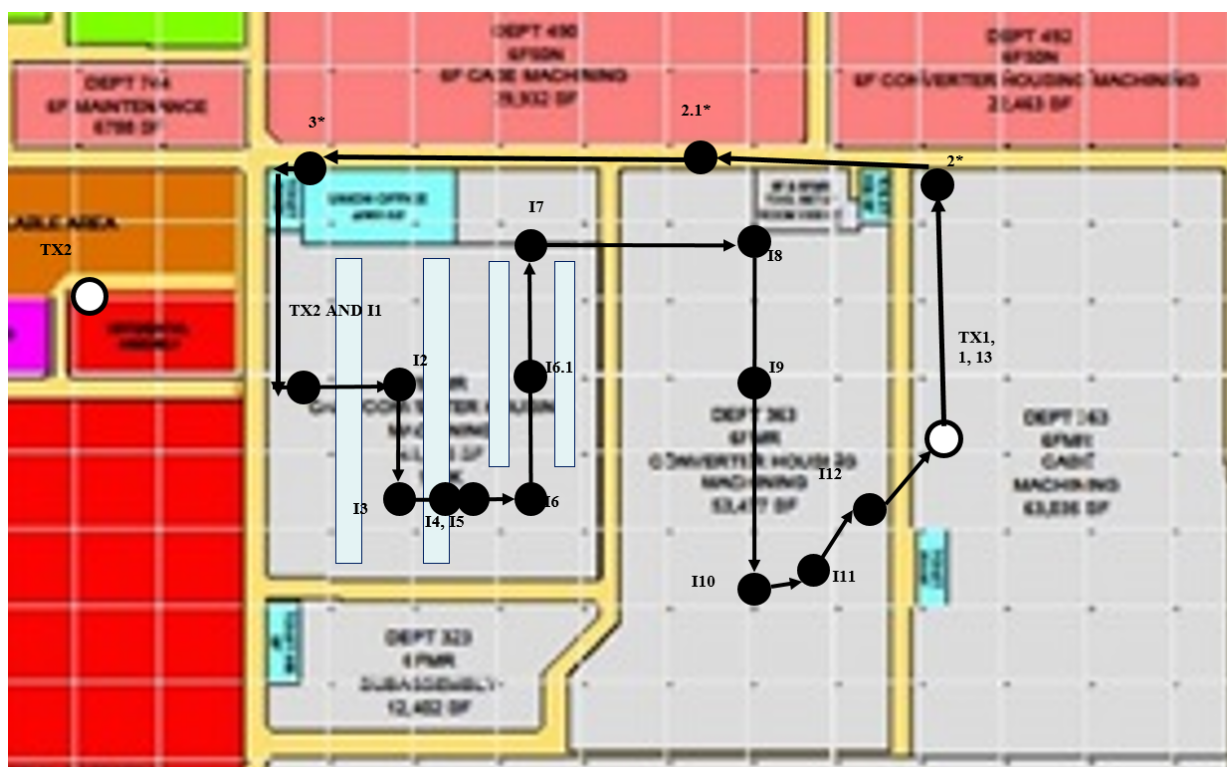

Figure A.2.2: Automotive Assembly Plant, Inner Loop Route. Each grid square represents 15.2 m x 15.2 m. 


\section{A.2.2 NIST Boulder Steam Plant}

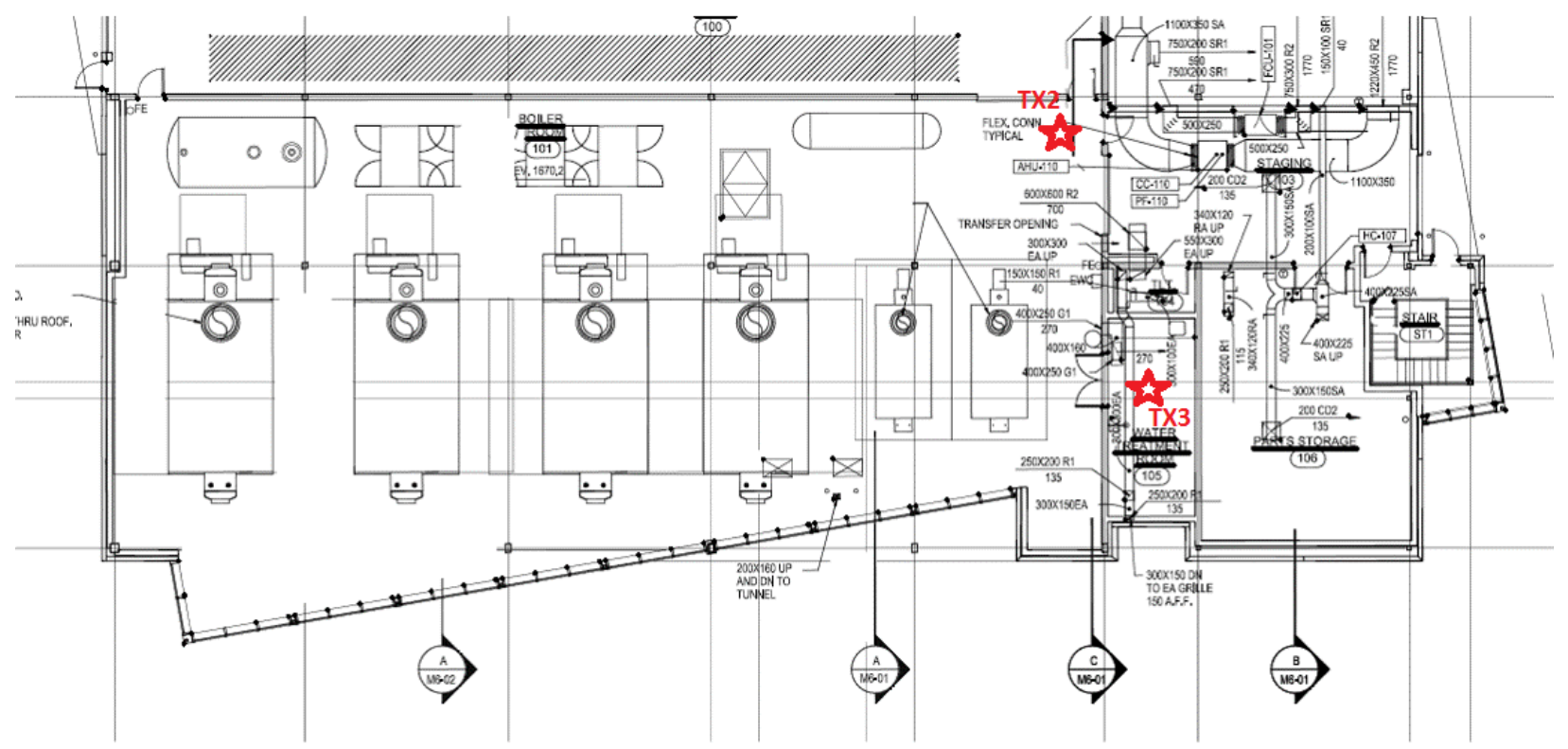

Figure A.2.3: Boulder Steam Plant, Floor Plan

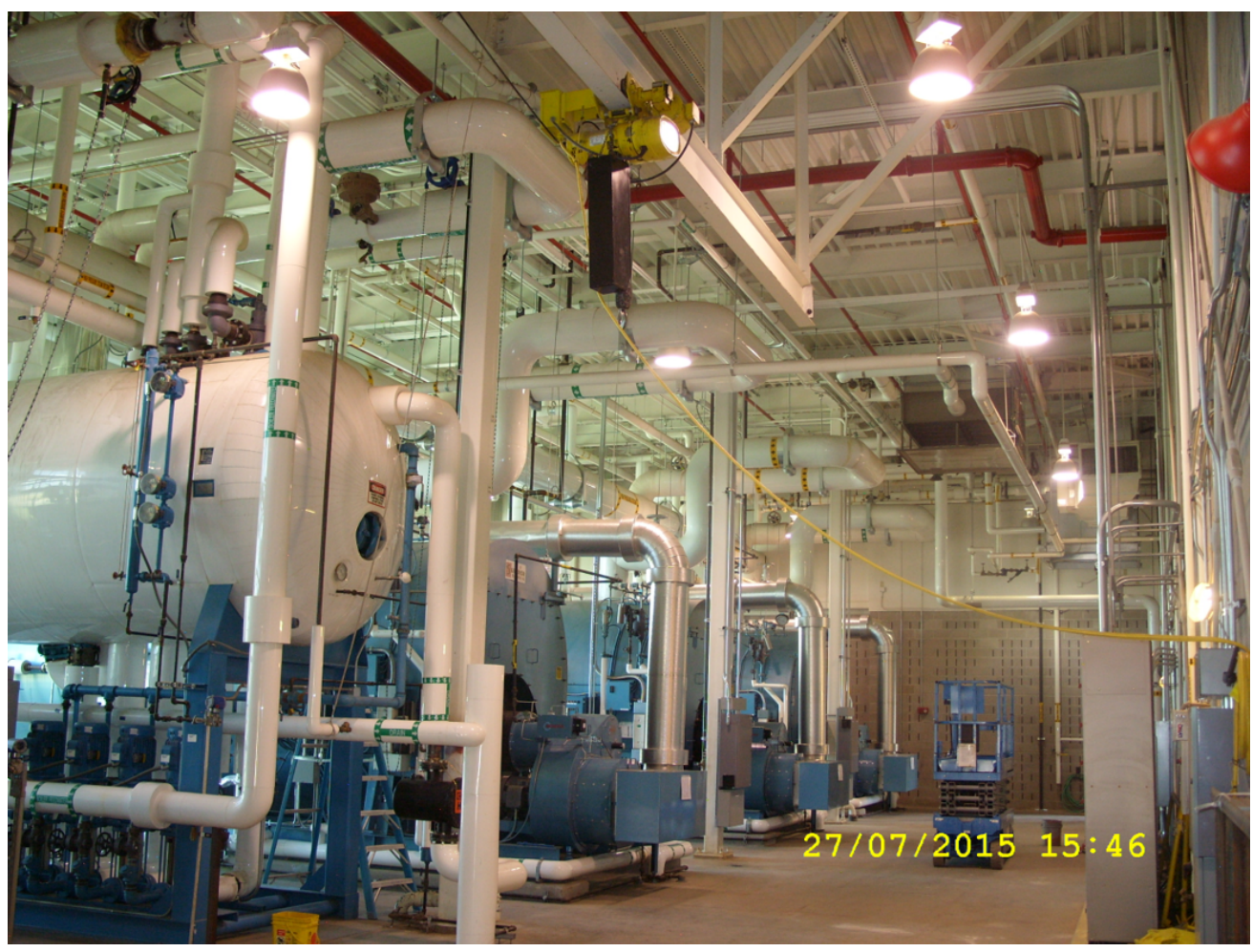

Figure A.2.4: Boulder Steam Plant, Visual 


\section{A.2.3 NIST Machine Shop}

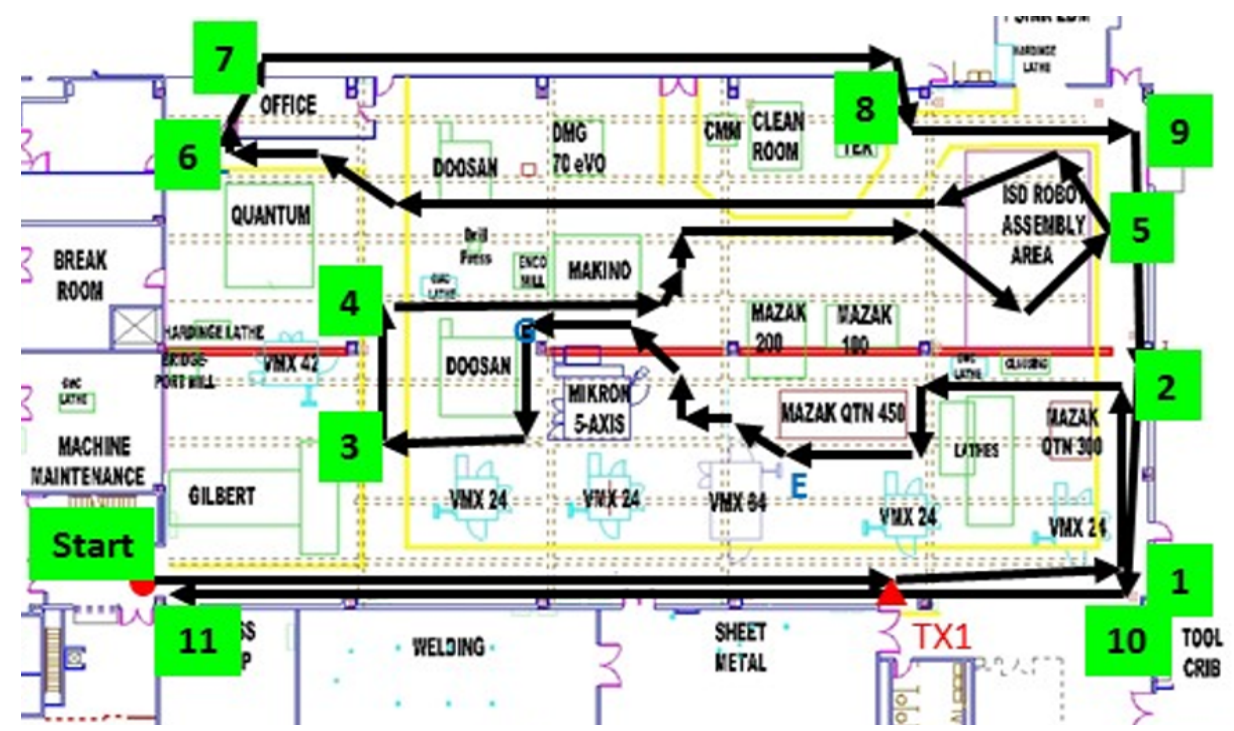

Figure A.2.5: NIST Machine Shop, Floor Plan

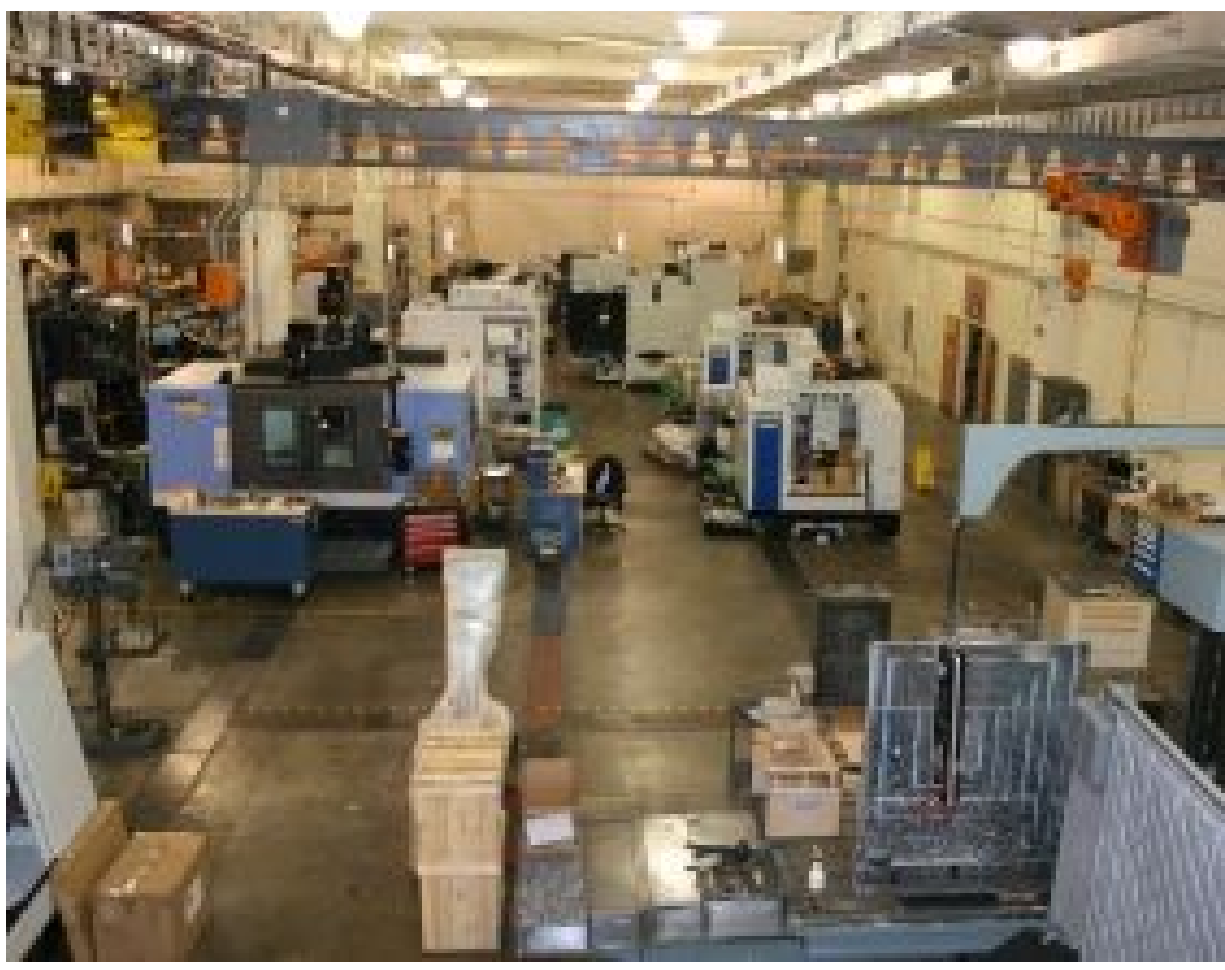

Figure A.2.6: NIST Machine Shop, Visual 


\section{A.2.4 NIST Open Area Test Site}

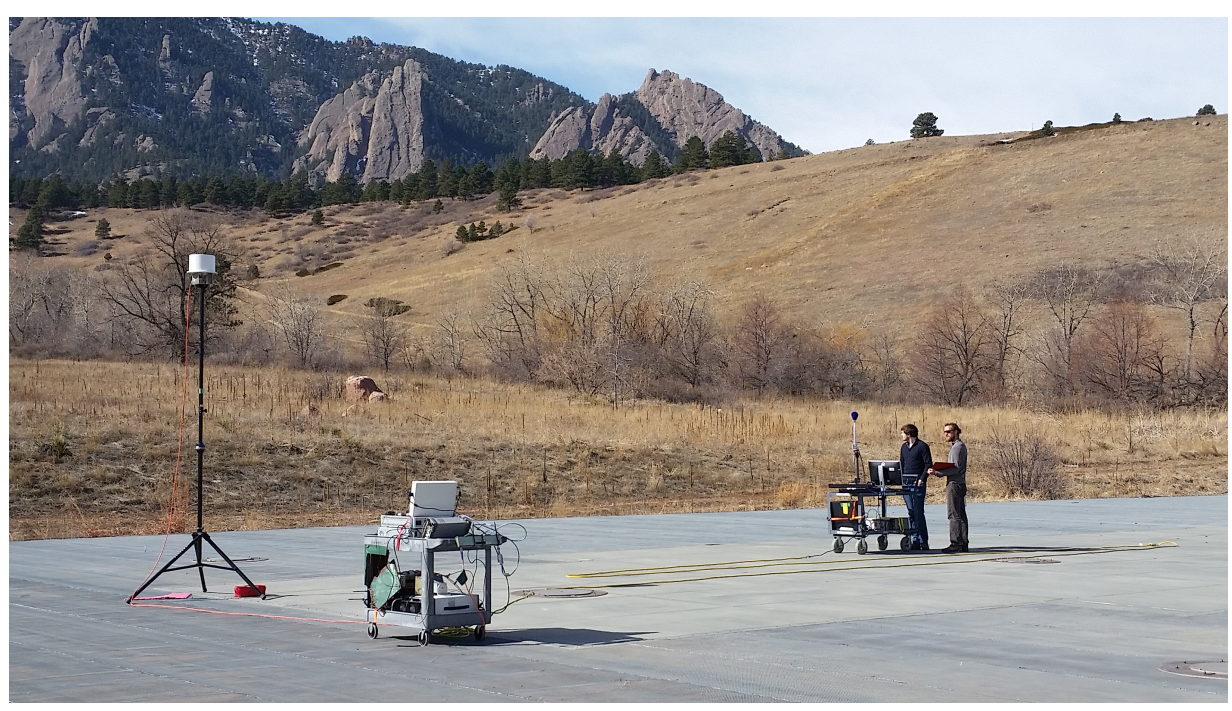

Figure A.2.7: NIST Open Area Test Site, Visual 


\section{A.3 Run Summaries}

A listing of expected channel metrics for each measurement run is listed in Tables A.1 and A.2. The expected value, $\mathbf{E}(\cdot)$, is defined as the first order moment, i.e., the mean, of the channel estimate given.

Table A.1: Expected Channel Estimates for All Runs: Part A. This table provides the frequency $(f)$, expected value of the path loss exponent $(\gamma)$, delay $(\tau)$, delay spread $(S)$, and $\mathrm{K}$-factor $(K)$, for a given measurement run.

\begin{tabular}{|c|c|c|c|c|c|}
\hline Run & $f(\mathbf{G H z})$ & $\mathbf{E}(\gamma)$ & $\mathbf{E}(\tau)(\mathbf{n s})$ & $\mathbf{E}(S)(\mathbf{n s})$ & $\mathbf{E}(K)(\mathbf{d B})$ \\
\hline \hline Oats 2G vpol 3115 run40 pp.mat & 2.2 & 1.7 & 43.9 & 6.2 & 24.0 \\
\hline Oats 2G vpol run39 pp.mat & 2.2 & 2.0 & 17.4 & 5.5 & 19.7 \\
\hline Oats 5G 3115horn vpol run36b pp.mat & 5.4 & 1.9 & 24.4 & 6.1 & 12.2 \\
\hline AAPlantD1 2GHz TX1 hpol run4 pp.mat & 2.2 & 5.4 & 559.3 & 106.6 & 0.7 \\
\hline AAPlantD1 2GHz TX1 vpol run3 pp.mat & 2.2 & 4.1 & 558.1 & 119.6 & 3.4 \\
\hline AAPlantD2 2GHz TX1 vpol internal runF pp.mat & 2.2 & 5.2 & 477.7 & 135.3 & 4.7 \\
\hline AAPlantD2 2GHz TX1 hpol internal runE pp.mat & 2.2 & 5.6 & 515.2 & 141.1 & 1.1 \\
\hline AAPlantD2 2GHz TX1 vpol internal runF pp.mat & 2.2 & 5.2 & 477.7 & 135.3 & 4.7 \\
\hline AAPlantD2 5GHz TX1 hpol internal runB pp.mat & 5.4 & 4.8 & 548.0 & 162.6 & 0.7 \\
\hline AAPlantD2 5GHz TX1 hpol run2 pp.mat & 5.4 & 5.0 & 703.0 & 179.1 & 1.4 \\
\hline AAPlantD2 5GHz TX1 vpol internal runA pp.mat & 5.4 & 5.3 & 527.5 & 154.4 & 3.5 \\
\hline AAPlantD2 5GHz TX1 vpol run1 pp.mat & 5.4 & 3.6 & 644.4 & 177.4 & 4.7 \\
\hline AAPlantD3 2GHz TX2b hpol internal run36 pp.mat & 2.2 & 6.4 & 492.1 & 135.1 & 1.5 \\
\hline AAPlantD3 2GHz TX2b vpol internal run33 pp.mat & 2.2 & 7.1 & 391.3 & 111.9 & 4.7 \\
\hline AAPlantD3 2GHz TX2b vpol run32 pp.mat & 2.2 & 3.7 & 642.3 & 121.7 & 4.2 \\
\hline AAPlantD3 2GHz Tx2b hpol run34 pp.mat & 2.2 & 4.2 & 538.6 & 114.2 & 1.9 \\
\hline AAPlantD3 5GHz TX2b hpol internal run39 pp.mat & 5.4 & 6.9 & 471.7 & 146.0 & 3.0 \\
\hline AAPlantD3 5GHz TX2b hpol run38 pp.mat & 5.4 & 4.0 & 552.3 & 138.6 & 4.3 \\
\hline AAPlantD3 5GHz TX2b vpol internal run40 pp.mat & 5.4 & 6.2 & 542.5 & 154.6 & 5.5 \\
\hline AAPlantD3 5GHz TX2b vpol run37 pp.mat & 5.4 & 3.1 & 630.7 & 153.2 & 5.2 \\
\hline GBurgD2 2GHz TX1 crossPol run3 pp.mat & 2.2 & 2.0 & 182.0 & 76.6 & 3.9 \\
\hline GBurgD2 2GHz TX1 longPol run2 pp.mat & 2.2 & 2.1 & 175.2 & 76.7 & 4.9 \\
\hline GBurgD2 2GHz TX1 vpol run1again pp.mat & 2.2 & 2.9 & 143.8 & 63.6 & 8.7 \\
\hline GBurgD2 5GHz TX1 crossPol run3 pp.mat & 5.4 & 2.0 & 183.7 & 75.2 & 5.0 \\
\hline GBurgD2 5GHz TX1 longPol run4 pp.mat & 5.4 & 2.2 & 185.2 & 73.6 & 4.3 \\
\hline GBurgD2 5GHz TX1 vpol run2 pp.mat & 5.4 & 2.7 & 135.4 & 54.7 & 9.3 \\
\hline GBurgD2 5GHz TX2high crossPol pp.mat & 5.4 & 1.1 & 168.5 & 78.3 & 4.2 \\
\hline
\end{tabular}


Table A.2: Expected Channel Estimates for All Runs: Part B. This table provides the frequency $(f)$, expected value of the path loss exponent $(\gamma)$, delay $(\tau)$, delay spread $(S)$, and $\mathrm{K}$-factor $(K)$, for a given measurement run.

\begin{tabular}{|c|c|c|c|c|c|}
\hline Run & $f(\mathbf{G H z})$ & $\mathbf{E}(\gamma)$ & $\mathbf{E}(\tau)(\mathbf{n s})$ & $\mathbf{E}(S)(\mathbf{n s})$ & $\mathbf{E}(K)(\mathbf{d B})$ \\
\hline \hline GBurgD2 5GHz TX2high longPol pp.mat & 5.4 & 1.2 & 170.8 & 77.4 & 4.4 \\
\hline GBurgD2 5GHz TX2low crossPol pp.mat & 5.4 & 1.1 & 1635.9 & 76.6 & 3.8 \\
\hline GBurgD2 5GHz TX2low longPol pp.mat & 5.4 & 1.3 & 70162.6 & 71.7 & 2.4 \\
\hline GBurgD2 5GHz TX2low vpol run1 pp.mat & 5.4 & 1.5 & 118.9 & 56.9 & 8.9 \\
\hline GBurgD3 2GHz TX2high crossPol run2 pp.mat & 2.2 & 1.5 & 186.9 & 79.3 & 5.1 \\
\hline GBurgD3 2GHz TX2high longPol run3 pp.mat & 2.2 & 1.6 & 184.6 & 80.2 & 4.9 \\
\hline GBurgD3 2GHz TX2low crossPol run2 pp.mat & 2.2 & 1.6 & 198.3 & 81.3 & 4.2 \\
\hline GBurgD3 2GHz TX2low longPol run3 pp.mat & 2.2 & 1.7 & 190.0 & 80.4 & 4.1 \\
\hline GBurgD3 2GHz TX2low vpol run1 pp.mat & 2.2 & 2.1 & 160.6 & 64.9 & 7.5 \\
\hline GburgD2 5GHz TX2high vpol pp.mat & 5.4 & 1.1 & 135.1 & 66.8 & 7.5 \\
\hline GburgD3 2GHz TX2high vpol run1 pp.mat & 2.2 & 1.6 & 155.2 & 65.7 & 8.7 \\
\hline Tx2 2G Boiler 3115Vpol run1 pp.mat & 2.2 & 2.3 & 69.7 & 32.1 & 9.5 \\
\hline Tx2 2G Boiler 3115Vpol run2 pp.mat & 2.2 & 2.8 & 94.1 & 34.5 & 9.1 \\
\hline Tx2 2G Boiler Vpol rrcos run3 pp.mat & 2.2 & 2.6 & 21578.5 & 37.7 & -10.2 \\
\hline Tx2 2G Boiler Vpol run2 pp.mat & 2.2 & 2.7 & 89.0 & 35.3 & 8.0 \\
\hline Tx2 2G Boiler Vpol run3 pp.mat & 2.2 & 2.5 & 90.6 & 35.4 & 8.3 \\
\hline Tx2 2G Boiler Vpol run4 pp.mat & 2.2 & 2.7 & 100.6 & 36.8 & 8.0 \\
\hline Tx2 5G Boiler 3115Vpol run1 pp.mat & 5.4 & 3.0 & 87.0 & 35.4 & 5.1 \\
\hline Tx2 5G Boiler 3115Xpol run1 pp.mat & 5.4 & 2.6 & 146.1 & 48.4 & -6.6 \\
\hline Tx2 5G Boiler Vpol run1 pp.mat & 5.4 & 3.5 & 92.7 & 30.9 & -1.0 \\
\hline Tx2 5G Boiler Xpol run1 pp.mat & 5.4 & 2.6 & 139.9 & 51.4 & -10.1 \\
\hline Tx2 5G Boiler 3115Vpol run1 pp.mat & 5.4 & 3.0 & 87.0 & 35.4 & 5.1 \\
\hline Tx3 2G Boiler 3115Vpol run2 pp.mat & 2.2 & 3.5 & 111.7 & 42.2 & -4.4 \\
\hline Tx3 5G 3115Vpol run2 pp.mat & 5.4 & 2.3 & 142.4 & 50.1 & -5.7 \\
\hline Tx3 5G 3115Xpol run1 pp.mat & 5.4 & 2.5 & 151.8 & 54.0 & -6.5 \\
\hline Tx3 5G Boiler Vpol run1 pp.mat & 5.4 & 2.8 & 144.2 & 50.7 & -9.3 \\
\hline Tx3 5G Boiler Xpol run1 pp.mat & 5.4 & 2.9 & 148.5 & 54.9 & -10.6 \\
\hline
\end{tabular}




\section{A.4 Detailed Results}

The following sections provide detailed information on each measurement dataset. For each dataset, the following information is provided:

Measurement Parameters This table provides the parameters of each measurement. Data includes the file name, the carrier frequency, antenna type, polarization, gain, and receiver sample rate. More information is contained within the meta-data section of the measurement files.

Table of Estimates This table provides mean, min, max, standard deviation (STD) and median absolute deviation (MAD). MAD is defined as the median value of the absolute deviation between a measured value in a population from its mean. Our measurements reported herein contain large outliers that skew standard deviation providing a false perspective of spread. MAD provides a more robust metric that is not heavily influenced by outliers. Outliers are identified as those data points that satisfy the iterative Grubb's test criteria with a significance threshold of 0.95 . Outlier information includes the number of elements in a data set and outlier counts at multiples of $\sigma$.

Gain Versus Acquisition Figure Provides a plot of the channel gain versus acquisition sorted by time stamp in ascending order.

Path Gain Versus Distance Provides a plot of the path gain versus the logarithm of distance. Path loss exponents are included with each figure. Free space path loss reference line is include for comparison.

Delay Spread Provides plots of the first and second order moments of delay spread. These metrics provide an indication of how diffuse the transmitted signal becomes during flight.

Delay Spread Provides plots of the first and second order moments of delay spread. These metrics provide an indication of how diffuse the transmitted signal becomes during flight. 


\section{A.4.1 AAPlantD1 2GHz TX1 hpol run4 pp.mat}

Table A.3: Measurement Parameters

\begin{tabular}{l|c}
\hline Parameter & Value \\
\hline \hline Mat File & AAPlantD1_2GHz_TX1_hpol_run4_pp.mat \\
Frequency $(\mathrm{GHz})$ & 2.245 \\
Location & AA Plant Day 1 at Automotive Assembly Plant \\
RX Antenna & Omni-directional, Cross Pol \\
RX Antenna Gain & -4.200 \\
TX Antenna & Omni-directional, V Pol \\
TX Antenna Gain & 2.900 \\
TX Power, Watts & 1.500 \\
PN Oversample Factor & 4.000 \\
Sample rate, MHz & 80.000
\end{tabular}

Table A.4: Statistics of Channel Estimates. Outliers are removed using a significance test of 0.95 .

\begin{tabular}{l||c|c|c|c||c|c||l}
\hline Estimate & $\min (\cdot)$ & $\operatorname{median}(\cdot)$ & $\operatorname{mean}(\cdot)$ & $\max (\cdot)$ & $\operatorname{std}(\cdot)$ & $\operatorname{mad}(\cdot)$ & outlier info \\
\hline \hline$K(\mathrm{~dB})$, NLOS & -19.9 & 0.1 & 0.7 & 20.3 & 4.4 & 3.2 & N: 22393 \\
\hline$K(\mathrm{~dB})$, LOS & 0.0 & 3.9 & 5.1 & 20.3 & 4.3 & 3.5 & N: 5689 \\
\hline$\tau(\mathrm{ns})$ & 53.8 & 569.8 & 559.3 & 1311.6 & 159.7 & 121.2 & N: 24823 \\
\hline$S(\mathrm{~ns})$ & 0.0 & 117.4 & 106.6 & 377.9 & 58.2 & 47.7 & N: 24766 \\
\hline
\end{tabular}

Table A.5: Statistics of Channel Estimates. Outliers are not removed. $\mathrm{N}$ indicates the total number of samples in the population. Outliers are provided at 10 and 20 times $\sigma$.

\begin{tabular}{|c|c|c|c|c|c|c|c|}
\hline Estimate & $\min (\cdot)$ & $\operatorname{median}(\cdot)$ & mean $(\cdot)$ & $\max (\cdot)$ & $\operatorname{std}(\cdot)$ & $\operatorname{mad}(\cdot)$ & outlier info \\
\hline$K(\mathrm{~dB}), \mathrm{NLOS}$ & -19.9 & 0.1 & 0.7 & 20.3 & 4.4 & 3.2 & $\mathrm{~N}: 22393$ \\
\hline$K(\mathrm{~dB}), \mathrm{LOS}$ & 0.0 & 3.9 & 5.1 & 20.3 & 4.3 & 3.5 & $\mathrm{~N}: 5689$ \\
\hline$\tau(\mathrm{ns})$ & 53.8 & 572.0 & 737.3 & 79337.5 & 2632.3 & 368.7 & $\begin{array}{l}\mathrm{N}: 25136 \\
10 \sigma(27060.0): 55 \\
20 \sigma(53382.7): 25\end{array}$ \\
\hline$S(\mathrm{~ns})$ & 0.0 & 118.5 & 255.0 & 39223.4 & 1588.3 & 292.5 & $\begin{array}{l}\mathrm{N}: 25136 \\
10 \sigma(16138.5): 88 \\
20 \sigma(32022.0): 7\end{array}$ \\
\hline
\end{tabular}




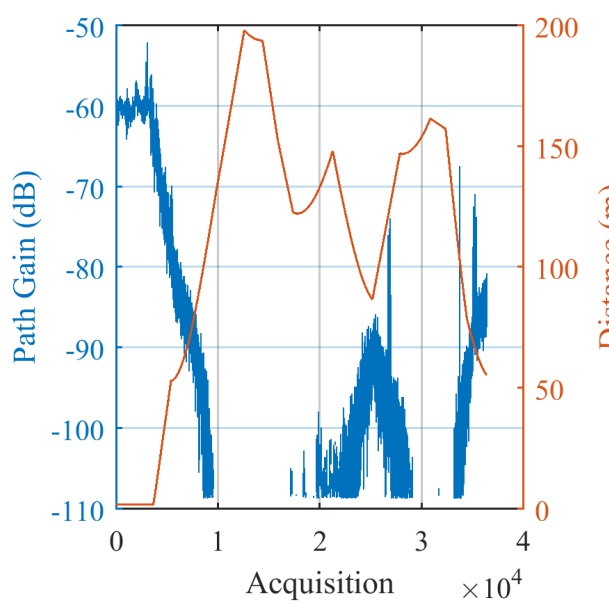

(a) Path Gain versus Acquisition Order

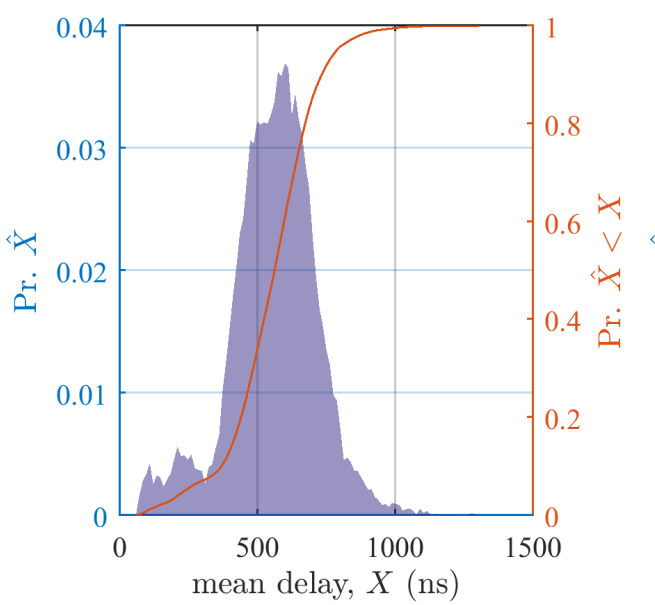

(c) Power Delay

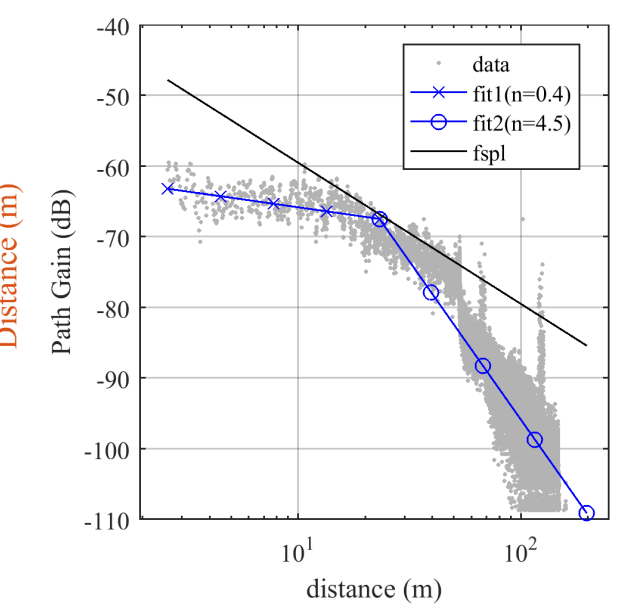

(b) Path Loss versus Distance

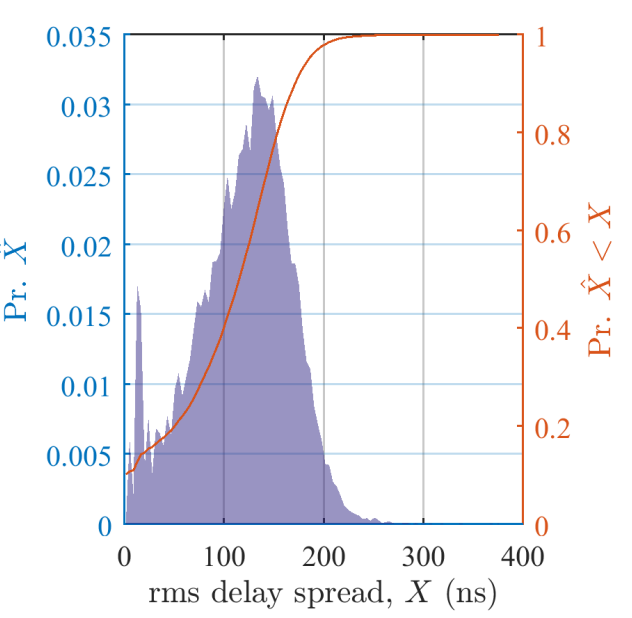

(d) Power Delay Spread

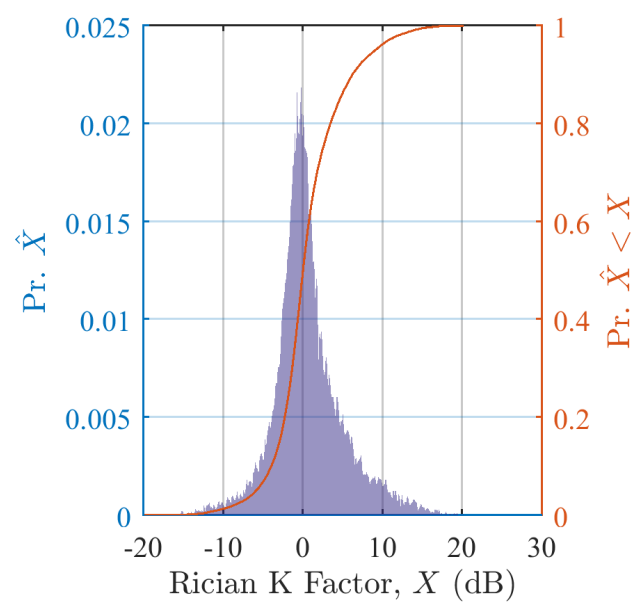

(e) Rician K-factor

Figure A.4.1: Channel characterizations for AAPlantD1 2GHz TX1 hpol run4 pp channel stats 


\section{A.4.2 AAPlantD1 2GHz TX1 vpol run3 pp.mat}

Table A.6: Measurement Parameters

\begin{tabular}{l|c}
\hline Parameter & Value \\
\hline \hline Mat File & AAPlantD1_2GHz_TX1_vpol_run3_pp.mat \\
Frequency $(\mathrm{GHz})$ & 2.245 \\
Location & AA Plant Day 1 at Automotive Assembly Plant \\
RX Antenna & Omni-directional, V Pol \\
RX Antenna Gain & -4.200 \\
TX Antenna & Omni-directional, V Pol \\
TX Antenna Gain & 2.900 \\
TX Power, Watts & 1.500 \\
PN Oversample Factor & 4.000 \\
Sample rate, MHz & 80.000
\end{tabular}

Table A.7: Statistics of Channel Estimates. Outliers are removed using a significance test of 0.95.

\begin{tabular}{l||c|c|c|c||c|c||l}
\hline Estimate & $\min (\cdot)$ & $\operatorname{median}(\cdot)$ & $\operatorname{mean}(\cdot)$ & $\max (\cdot)$ & $\operatorname{std}(\cdot)$ & $\operatorname{mad}(\cdot)$ & outlier info \\
\hline \hline$K(\mathrm{~dB})$, NLOS & -17.6 & 2.4 & 3.4 & 26.1 & 6.1 & 4.8 & N: 31305 \\
\hline$K(\mathrm{~dB})$, LOS & 0.0 & 8.2 & 8.8 & 26.1 & 4.9 & 4.0 & N: 12696 \\
\hline$\tau(\mathrm{ns})$ & 21.2 & 576.0 & 558.1 & 1364.1 & 168.6 & 127.0 & $\mathrm{~N}: 31610$ \\
\hline$S(\mathrm{~ns})$ & 0.0 & 125.8 & 119.6 & 334.2 & 47.8 & 38.3 & $\mathrm{~N}: 31486$ \\
\hline
\end{tabular}

Table A.8: Statistics of Channel Estimates. Outliers are not removed. $\mathrm{N}$ indicates the total number of samples in the population. Outliers are provided at 10 and 20 times $\sigma$.

\begin{tabular}{|c|c|c|c|c|c|c|c|}
\hline Estimate & $\min (\cdot)$ & $\operatorname{median}(\cdot)$ & mean $(\cdot)$ & $\max (\cdot)$ & $\operatorname{std}(\cdot)$ & $\operatorname{mad}(\cdot)$ & outlier info \\
\hline$K(\mathrm{~dB}), \mathrm{NLOS}$ & -17.6 & 2.4 & 3.4 & 26.1 & 6.1 & 4.8 & $\mathrm{~N}: 31305$ \\
\hline$K(\mathrm{~dB}), \operatorname{LOS}$ & 0.0 & 8.2 & 8.8 & 26.1 & 4.9 & 4.0 & $\mathrm{~N}: 12696$ \\
\hline$\tau(\mathrm{ns})$ & 21.2 & 576.9 & 608.4 & 71350.0 & 1459.7 & 178.2 & $\begin{array}{l}\mathrm{N}: 31769 \\
10 \sigma(15205.8): 30 \\
20 \sigma(29803.1): 23\end{array}$ \\
\hline$S(\mathrm{~ns})$ & 0.0 & 126.3 & 172.8 & 36164.9 & 760.7 & 109.7 & $\begin{array}{l}\mathrm{N}: 31769 \\
10 \sigma(7779.4): 71 \\
20 \sigma(15386.0): 13\end{array}$ \\
\hline
\end{tabular}




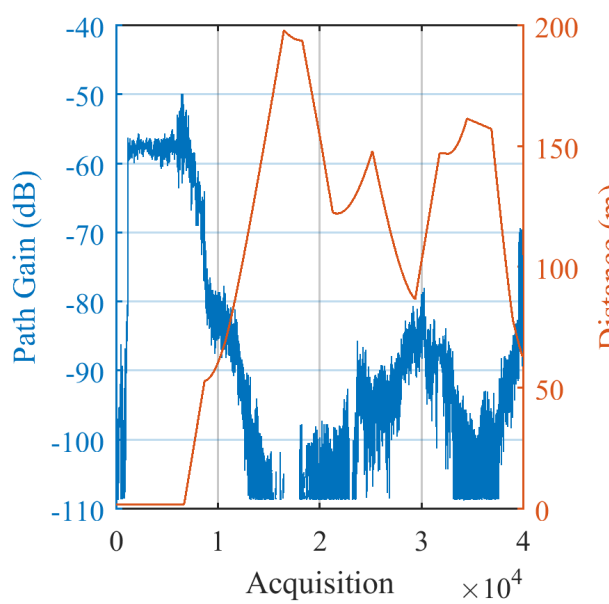

(a) Path Gain versus Acquisition Order

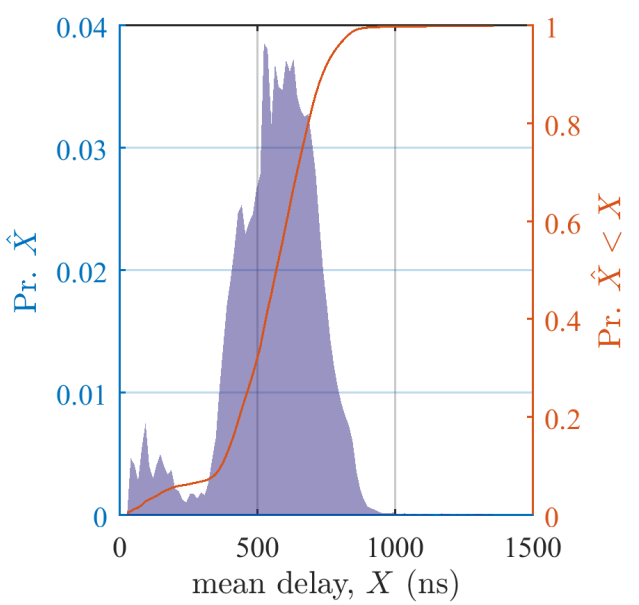

(c) Power Delay

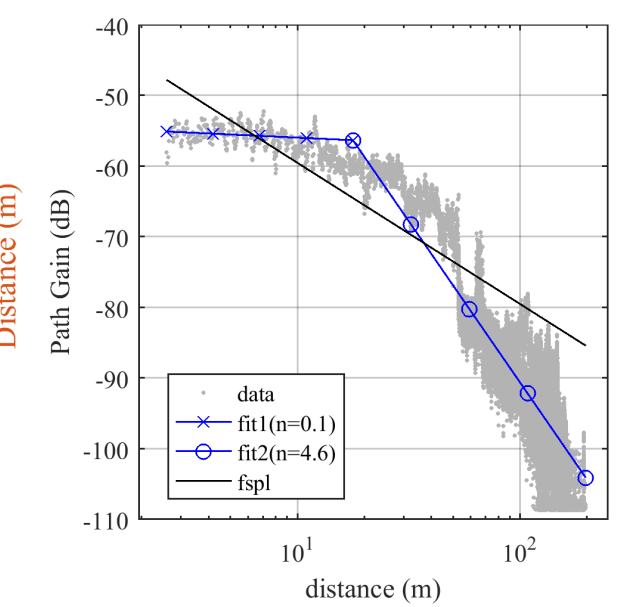

(b) Path Loss versus Distance

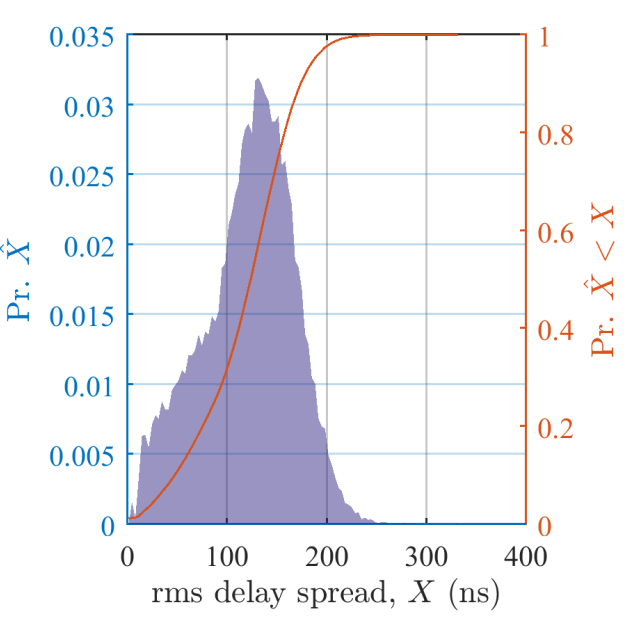

(d) Power Delay Spread

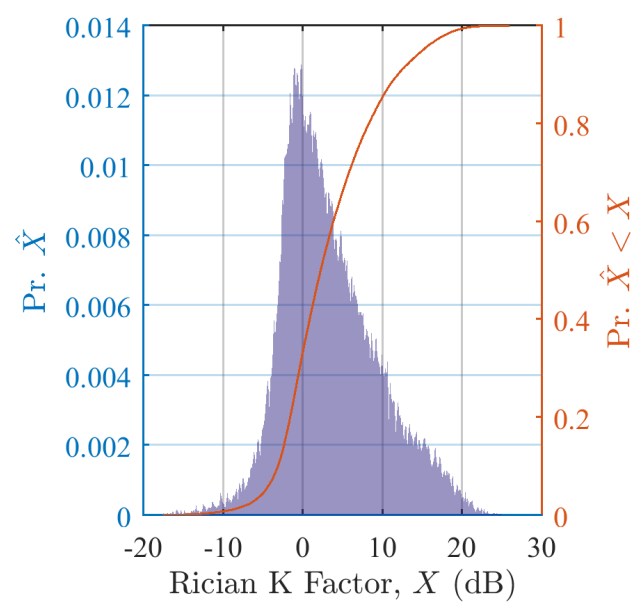

(e) Rician K-factor

Figure A.4.2: Channel characterizations for AAPlantD1 2GHz TX1 vpol run3 pp channel stats 


\section{A.4.3 AAPlantD2 2GHz TX1 vpol internal runF pp.mat}

Table A.9: Measurement Parameters

\begin{tabular}{l|c}
\hline Parameter & Value \\
\hline \hline Mat File & AAPlantD2 2GHz TX1 vpol internal runF_pp.mat \\
Frequency (GHz) & 2.245 \\
Location & AA Plant Day 2 at Automotive Assembly Plant \\
RX Antenna & Omni-directional, V Pol \\
RX Antenna Gain & -4.200 \\
TX Antenna & Omni-directional, V Pol \\
TX Antenna Gain & 2.900 \\
TX Power, Watts & 1.500 \\
PN Oversample Factor & 4.000 \\
Sample rate, MHz & 80.000
\end{tabular}

Table A.10: Statistics of Channel Estimates. Outliers are removed using a significance test of 0.95.

\begin{tabular}{l||c|c|c|c||c|c||l}
\hline Estimate & $\min (\cdot)$ & $\operatorname{median}(\cdot)$ & $\operatorname{mean}(\cdot)$ & $\max (\cdot)$ & $\operatorname{std}(\cdot)$ & $\operatorname{mad}(\cdot)$ & outlier info \\
\hline \hline$K(\mathrm{~dB})$, NLOS & -21.0 & 5.6 & 4.7 & 26.1 & 6.6 & 4.9 & N: 26183 \\
\hline$K(\mathrm{~dB})$, LOS & 1.9 & 9.1 & 9.7 & 23.9 & 3.4 & 2.7 & N: 11645 \\
\hline$\tau(\mathrm{ns})$ & 40.8 & 480.8 & 477.7 & 1159.3 & 143.2 & 103.0 & N: 26043 \\
\hline$S(\mathrm{~ns})$ & 13.9 & 139.2 & 135.3 & 314.0 & 38.4 & 29.3 & N: 24872 \\
\hline
\end{tabular}

Table A.11: Statistics of Channel Estimates. Outliers are not removed. N indicates the total number of samples in the population. Outliers are provided at 10 and 20 times $\sigma$.

\begin{tabular}{l||c|c|c|c||c|c||l}
\hline Estimate & $\min (\cdot)$ & $\operatorname{median}(\cdot)$ & $\operatorname{mean}(\cdot)$ & $\max (\cdot)$ & $\operatorname{std}(\cdot)$ & $\operatorname{mad}(\cdot)$ & outlier info \\
\hline \hline$K(\mathrm{~dB})$, NLOS & -21.0 & 5.6 & 4.7 & 26.1 & 6.6 & 4.9 & $\mathrm{~N}: 26183$ \\
\hline$K(\mathrm{~dB})$, LOS & 1.9 & 9.1 & 9.7 & 26.1 & 3.4 & 2.7 & $\mathrm{~N}: 11647$ \\
\hline$\tau(\mathrm{ns})$ & 40.8 & 481.7 & 484.1 & 4797.2 & 174.4 & 108.8 & $\mathrm{~N}: 26183$ \\
& & & & & & & $10 \sigma(2228.4): 15$ \\
& & & & & & & $20 \sigma(3972.7): 3$ \\
\hline$S(\mathrm{~ns})$ & 13.9 & 141.4 & 293.3 & 15686.3 & 832.6 & 300.2 & $\mathrm{~N}: 26183$ \\
& & & & & & & $10 \sigma(8619.1): 25$ \\
\hline
\end{tabular}




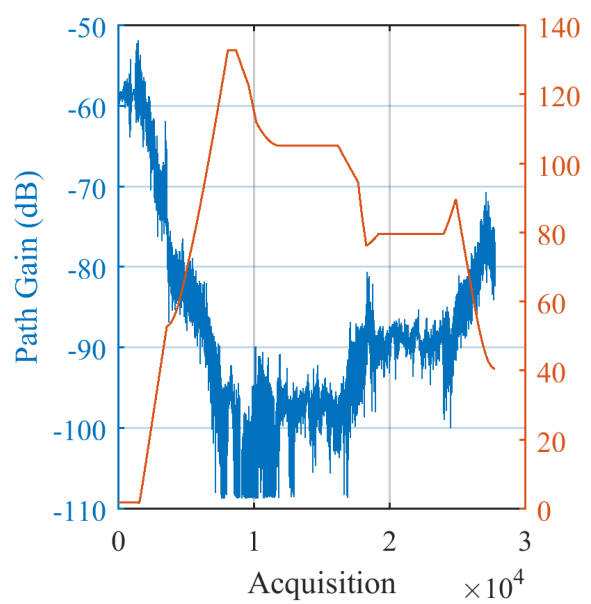

(a) Path Gain versus Acquisition Order

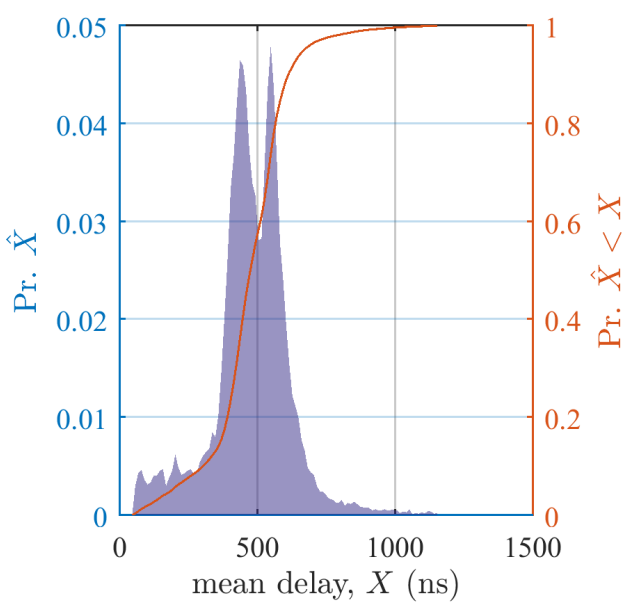

(c) Power Delay

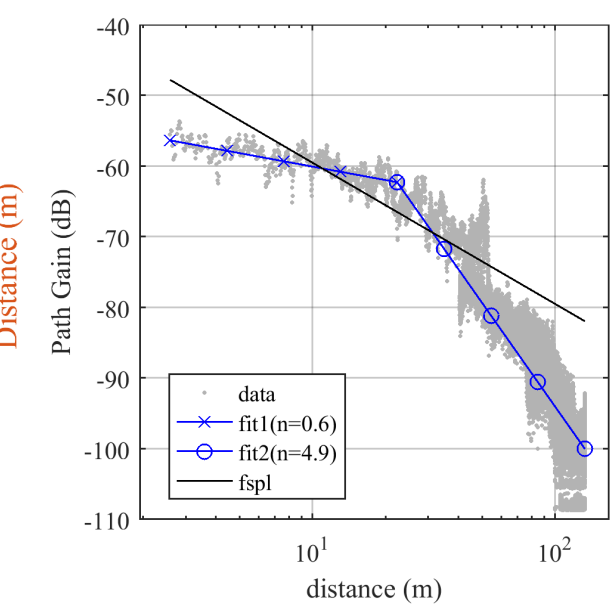

(b) Path Loss versus Distance

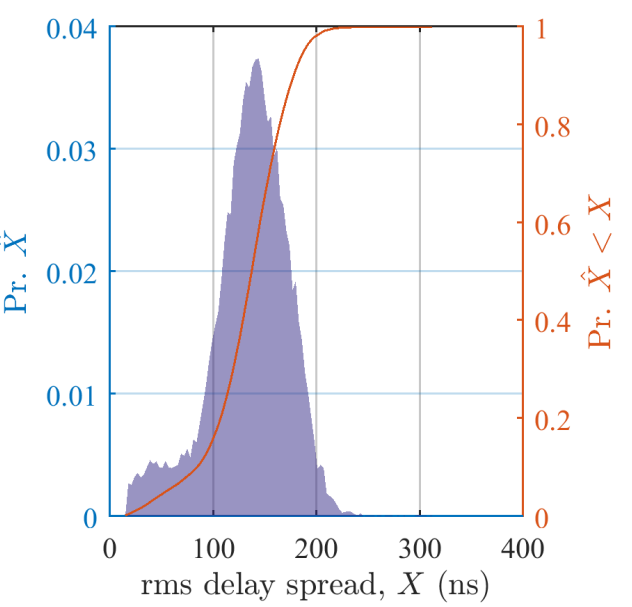

(d) Power Delay Spread

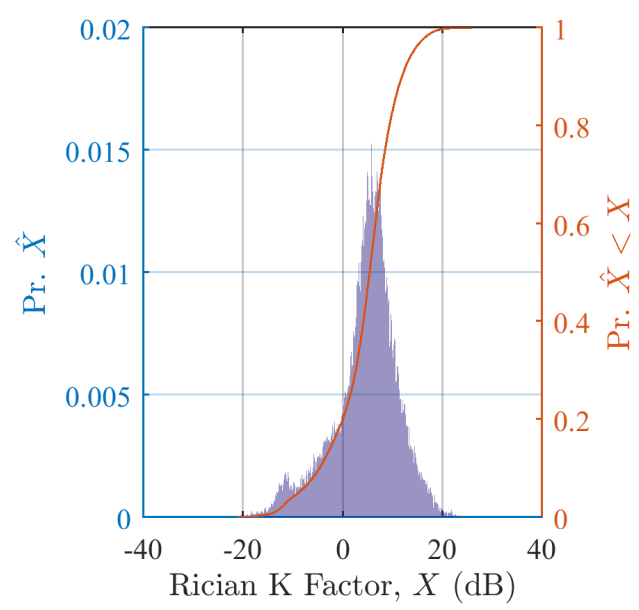

(e) Rician K-factor

Figure A.4.3: Channel characterizations for AAPlantD2 2GHz TX1 vpol internal runF pp channel stats 


\section{A.4.4 AAPlantD2 2GHz TX1 hpol internal runE pp.mat}

Table A.12: Measurement Parameters

\begin{tabular}{l|c}
\hline Parameter & Value \\
\hline \hline Mat File & AAPlantD2_2GHz_TX1_hpol_internal_runE_pp.mat \\
Frequency $(\mathrm{GHz})$ & 2.245 \\
Location & AA Plant Day 2 at Automotive Assembly Plant \\
RX Antenna & Omni-directional, Cross Pol \\
RX Antenna Gain & -4.200 \\
TX Antenna & Omni-directional, V Pol \\
TX Antenna Gain & 2.900 \\
TX Power, Watts & 1.500 \\
PN Oversample Factor & 4.000 \\
Sample rate, MHz & 80.000
\end{tabular}

Table A.13: Statistics of Channel Estimates. Outliers are removed using a significance test of 0.95.

\begin{tabular}{l||c|c|c|c||c|c||l}
\hline Estimate & $\min (\cdot)$ & $\operatorname{median}(\cdot)$ & $\operatorname{mean}(\cdot)$ & $\max (\cdot)$ & $\operatorname{std}(\cdot)$ & $\operatorname{mad}(\cdot)$ & outlier info \\
\hline \hline$K(\mathrm{~dB})$, NLOS & -23.0 & 1.6 & 1.1 & 22.4 & 5.7 & 4.6 & N: 26990 \\
\hline$K(\mathrm{~dB})$, LOS & 0.3 & 7.1 & 7.6 & 20.7 & 3.1 & 2.5 & N: 5920 \\
\hline$\tau(\mathrm{ns})$ & 28.1 & 510.5 & 515.2 & 1256.3 & 155.6 & 112.6 & N: 26502 \\
\hline$S(\mathrm{~ns})$ & 0.0 & 142.6 & 141.1 & 282.3 & 31.1 & 24.0 & N: 25175 \\
\hline
\end{tabular}

Table A.14: Statistics of Channel Estimates. Outliers are not removed. N indicates the total number of samples in the population. Outliers are provided at 10 and 20 times $\sigma$.

\begin{tabular}{|c|c|c|c|c|c|c|c|}
\hline Estimate & $\min (\cdot)$ & $\operatorname{median}(\cdot)$ & mean $(\cdot)$ & $\max (\cdot)$ & $\operatorname{std}(\cdot)$ & $\operatorname{mad}(\cdot)$ & outlier info \\
\hline$K(\mathrm{~dB})$, NLOS & -23.0 & 1.6 & 1.1 & 22.4 & 5.7 & 4.6 & $\mathrm{~N}: 26990$ \\
\hline$K(\mathrm{~dB}), \operatorname{LOS}$ & 0.3 & 7.1 & 7.6 & 22.4 & 3.2 & 2.5 & $\mathrm{~N}: 5922$ \\
\hline$\tau(\mathrm{ns})$ & 28.1 & 514.1 & 544.9 & 15423.9 & 320.4 & 142.4 & $\begin{array}{l}\mathrm{N}: 26993 \\
10 \sigma(3749.4): 36 \\
20 \sigma(6953.9): 5\end{array}$ \\
\hline$S(\mathrm{~ns})$ & 0.0 & 145.0 & 424.6 & 29126.7 & 1332.9 & 529.0 & $\begin{array}{l}\mathrm{N}: 26993 \\
10 \sigma(13753.6): 27 \\
20 \sigma(27082.6): 1\end{array}$ \\
\hline
\end{tabular}




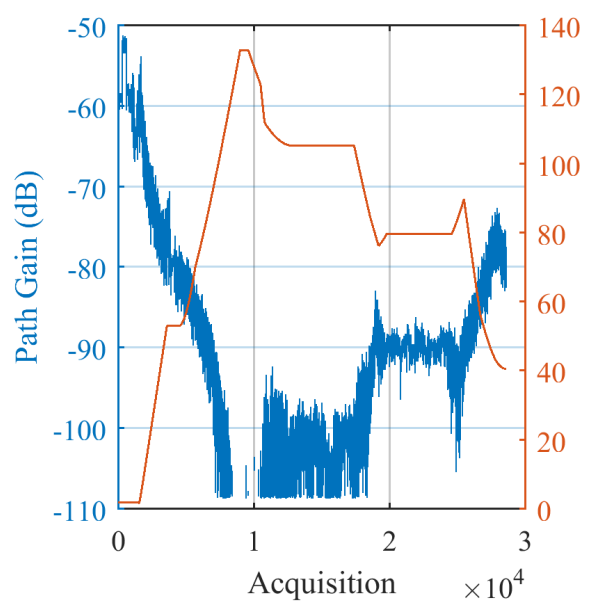

(a) Path Gain versus Acquisition Order

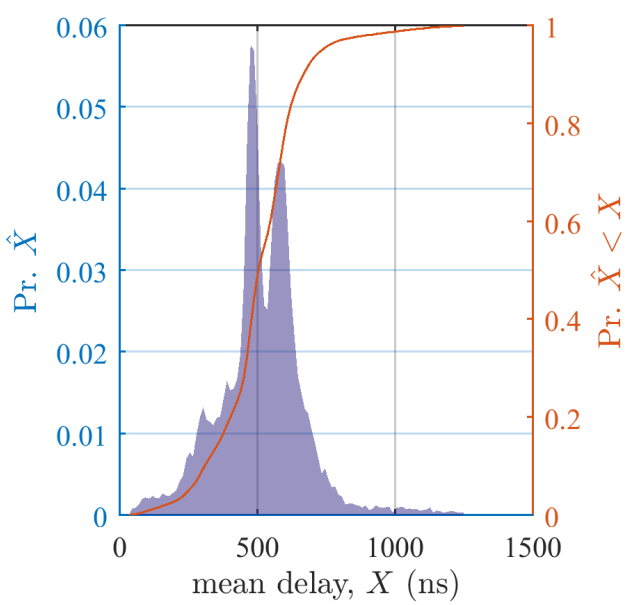

(c) Power Delay

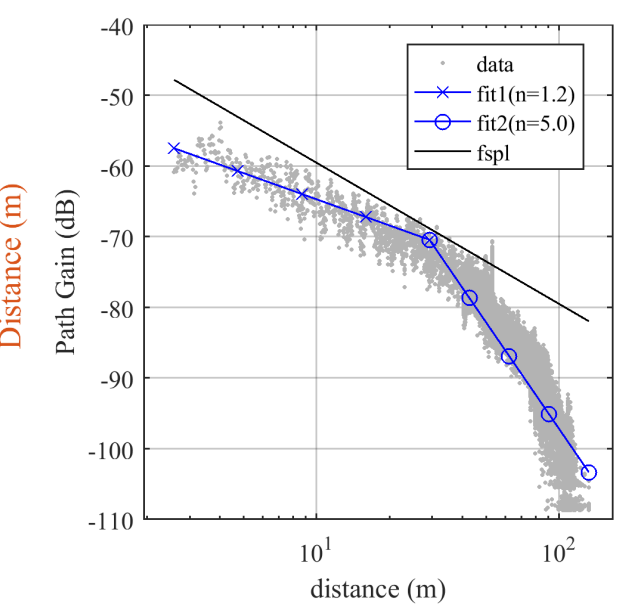

(b) Path Loss versus Distance

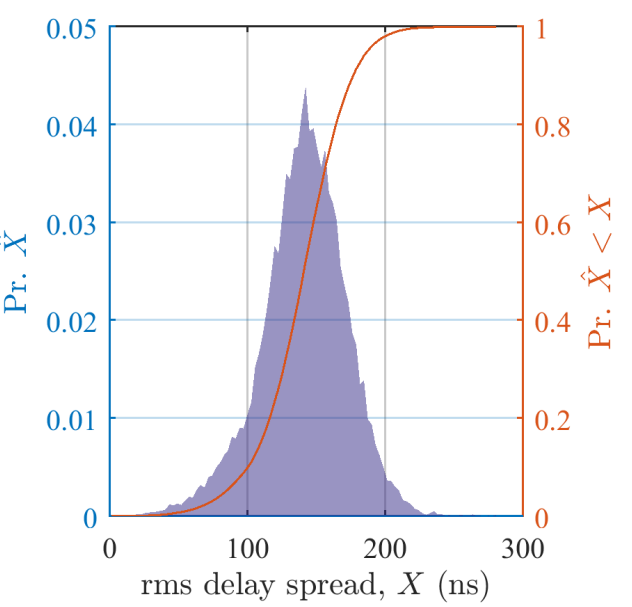

(d) Power Delay Spread

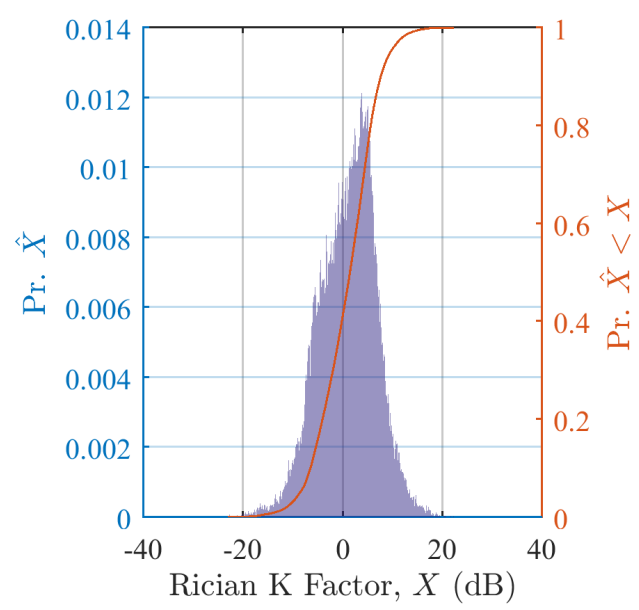

(e) Rician K-factor

Figure A.4.4: Channel characterizations for AAPlantD2 2GHz TX1 hpol internal runE pp channel stats 


\section{A.4.5 AAPlantD2 2GHz TX1 vpol internal runF pp.mat}

Table A.15: Measurement Parameters

\begin{tabular}{l|c}
\hline Parameter & Value \\
\hline \hline Mat File & AAPlantD2_2GHz_TX1_vpol_interna \\
Frequency (GHz) & 2.245 \\
Location & AA Plant Day 2 at Automotive Ass \\
RX Antenna & Omni-directional, V Pol \\
RX Antenna Gain & -4.200 \\
TX Antenna & Omni-directional, V Pol \\
TX Antenna Gain & 2.900 \\
TX Power, Watts & 1.500 \\
PN Oversample Factor & 4.000 \\
Sample rate, MHz & 80.000
\end{tabular}

Table A.16: Statistics of Channel Estimates. Outliers are removed using a significance test of 0.95.

\begin{tabular}{l||c|c|c|c||c|c||l}
\hline Estimate & $\min (\cdot)$ & $\operatorname{median}(\cdot)$ & $\operatorname{mean}(\cdot)$ & $\max (\cdot)$ & $\operatorname{std}(\cdot)$ & $\operatorname{mad}(\cdot)$ & outlier info \\
\hline \hline$K(\mathrm{~dB})$, NLOS & -21.0 & 5.6 & 4.7 & 26.1 & 6.6 & 4.9 & N: 26183 \\
\hline$K(\mathrm{~dB})$, LOS & 1.9 & 9.1 & 9.7 & 23.9 & 3.4 & 2.7 & N: 11645 \\
\hline$\tau(\mathrm{ns})$ & 40.8 & 480.8 & 477.7 & 1159.3 & 143.2 & 103.0 & N: 26043 \\
\hline$S(\mathrm{~ns})$ & 13.9 & 139.2 & 135.3 & 314.0 & 38.4 & 29.3 & N: 24872 \\
\hline
\end{tabular}

Table A.17: Statistics of Channel Estimates. Outliers are not removed. N indicates the total number of samples in the population. Outliers are provided at 10 and 20 times $\sigma$.

\begin{tabular}{l||c|c|c|c||c|c||l}
\hline Estimate & $\min (\cdot)$ & $\operatorname{median}(\cdot)$ & $\operatorname{mean}(\cdot)$ & $\max (\cdot)$ & $\operatorname{std}(\cdot)$ & $\operatorname{mad}(\cdot)$ & outlier info \\
\hline \hline$K(\mathrm{~dB})$, NLOS & -21.0 & 5.6 & 4.7 & 26.1 & 6.6 & 4.9 & $\mathrm{~N}: 26183$ \\
\hline$K(\mathrm{~dB})$, LOS & 1.9 & 9.1 & 9.7 & 26.1 & 3.4 & 2.7 & $\mathrm{~N}: 11647$ \\
\hline$\tau(\mathrm{ns})$ & 40.8 & 481.7 & 484.1 & 4797.2 & 174.4 & 108.8 & $\mathrm{~N}: 26183$ \\
& & & & & & & $10 \sigma(2228.4): 15$ \\
& & & & & & & $20 \sigma(3972.7): 3$ \\
\hline$S(\mathrm{~ns})$ & 13.9 & 141.4 & 293.3 & 15686.3 & 832.6 & 300.2 & $\mathrm{~N}: 26183$ \\
& & & & & & & $10 \sigma(8619.1): 25$ \\
\hline
\end{tabular}




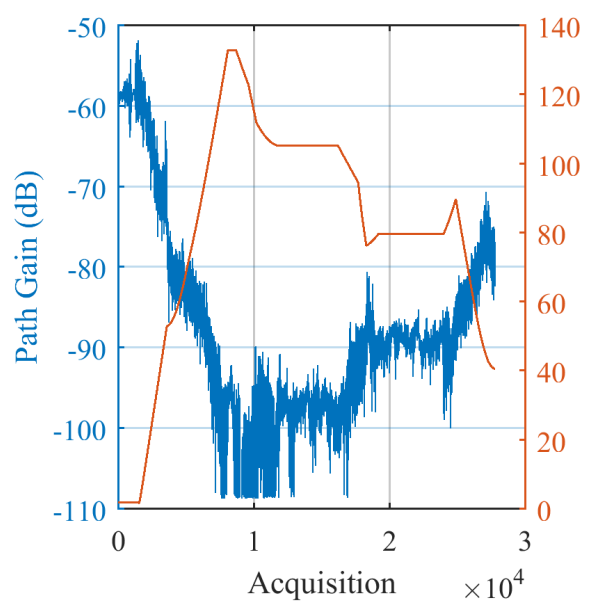

(a) Path Gain versus Acquisition Order

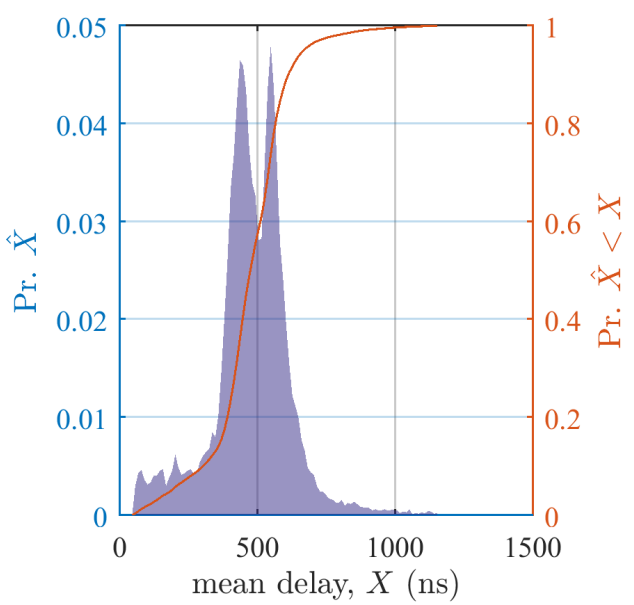

(c) Power Delay

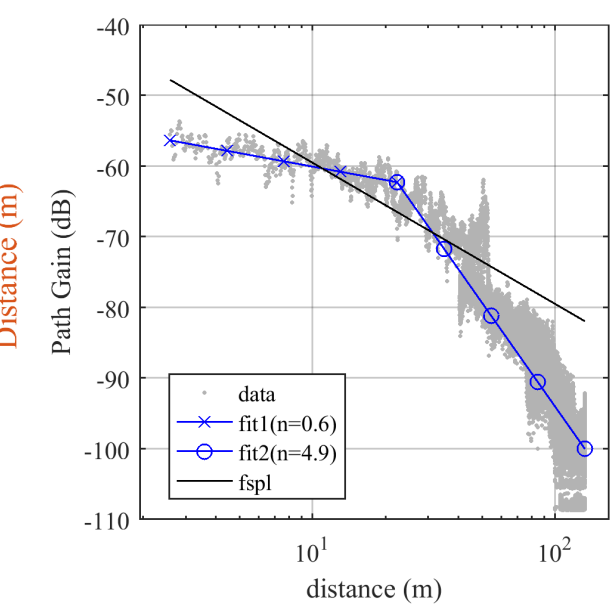

(b) Path Loss versus Distance

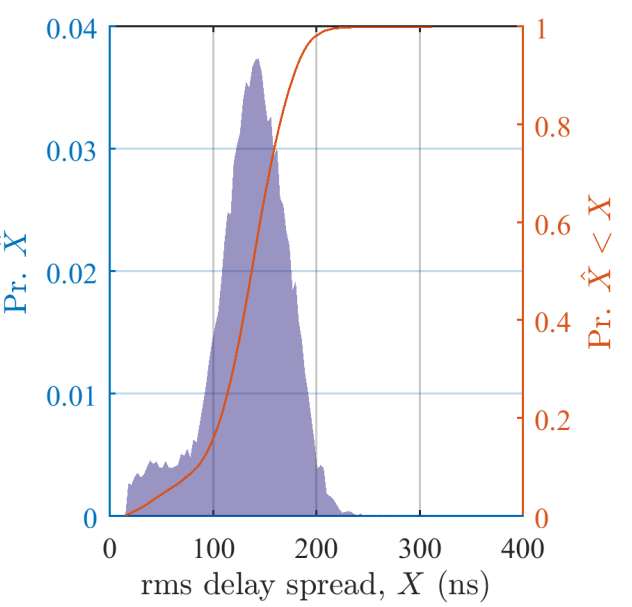

(d) Power Delay Spread

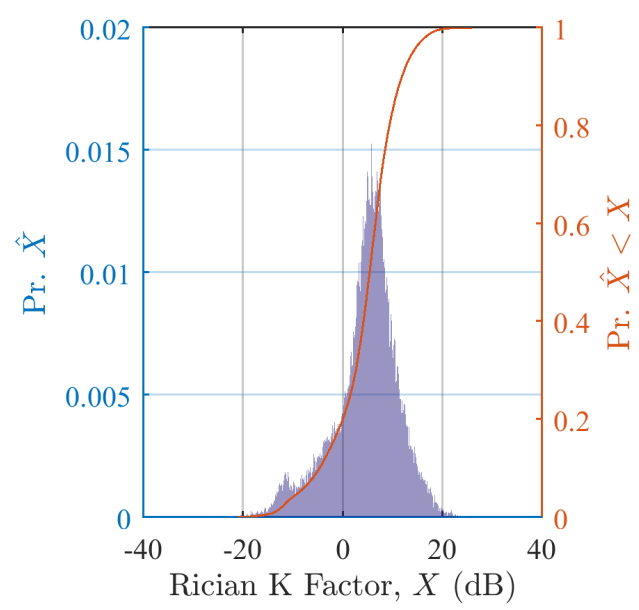

(e) Rician K-factor

Figure A.4.5: Channel characterizations for AAPlantD2 2GHz TX1 vpol internal runF pp channel stats 


\section{A.4.6 AAPlantD2 5GHz TX1 hpol internal runB pp.mat}

Table A.18: Measurement Parameters

\begin{tabular}{l|c}
\hline Parameter & Value \\
\hline \hline Mat File & AAPlantD2_5GHz_TX1_hpol_internal_runB_pp.mat \\
Frequency $(\mathrm{GHz})$ & 5.400 \\
Location & AA Plant Day 2 at Automotive Assembly Plant \\
RX Antenna & Omni-directional, Cross Pol \\
RX Antenna Gain & -3.500 \\
TX Antenna & Omni-directional, V Pol \\
TX Antenna Gain & 3.600 \\
TX Power, Watts & 1.250 \\
PN Oversample Factor & 4.000 \\
Sample rate, MHz & 80.000
\end{tabular}

Table A.19: Statistics of Channel Estimates. Outliers are removed using a significance test of 0.95.

\begin{tabular}{l||c|c|c|c||c|c||l}
\hline Estimate & $\min (\cdot)$ & $\operatorname{median}(\cdot)$ & $\operatorname{mean}(\cdot)$ & $\max (\cdot)$ & $\operatorname{std}(\cdot)$ & $\operatorname{mad}(\cdot)$ & outlier info \\
\hline \hline$K(\mathrm{~dB})$, NLOS & -23.4 & 2.7 & 0.7 & 21.4 & 8.0 & 6.8 & N: 24833 \\
\hline$K(\mathrm{~dB})$, LOS & 3.8 & 9.6 & 9.9 & 20.3 & 2.4 & 1.9 & N: 5169 \\
\hline$\tau(\mathrm{ns})$ & 66.1 & 550.7 & 548.0 & 1255.5 & 153.3 & 119.0 & N: 24819 \\
\hline$S(\mathrm{~ns})$ & 15.6 & 163.5 & 162.6 & 362.7 & 42.3 & 33.2 & N: 24561 \\
\hline
\end{tabular}

Table A.20: Statistics of Channel Estimates. Outliers are not removed. N indicates the total number of samples in the population. Outliers are provided at 10 and 20 times $\sigma$.

\begin{tabular}{l||c|c|c|c||c|c||l}
\hline Estimate & $\min (\cdot)$ & $\operatorname{median}(\cdot)$ & $\operatorname{mean}(\cdot)$ & $\max (\cdot)$ & $\operatorname{std}(\cdot)$ & $\operatorname{mad}(\cdot)$ & outlier info \\
\hline \hline$K(\mathrm{~dB})$, NLOS & -23.4 & 2.7 & 0.7 & 21.4 & 8.0 & 6.8 & $\mathrm{~N}: 24833$ \\
\hline$K(\mathrm{~dB})$, LOS & 3.8 & 9.6 & 9.9 & 21.4 & 2.4 & 1.9 & $\mathrm{~N}: 5172$ \\
\hline$\tau(\mathrm{ns})$ & 66.1 & 550.9 & 548.5 & 2156.8 & 155.1 & 119.4 & $\mathrm{~N}: 24833$ \\
& & & & & & & $10 \sigma(2099.4): 2$ \\
\hline$S(\mathrm{~ns})$ & 15.6 & 164.0 & 191.1 & 9965.6 & 322.4 & 68.2 & $\mathrm{~N}: 24833$ \\
& & & & & & & \\
& & & & & & & $20 \sigma(3415.4): 83$ \\
& & & & & & &
\end{tabular}




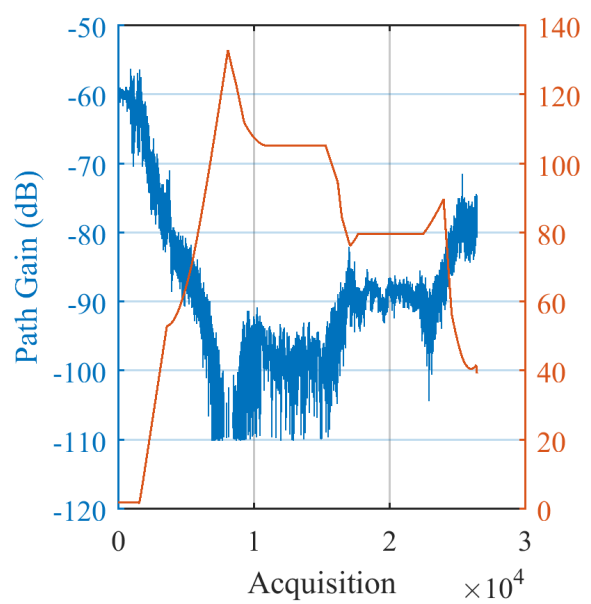

(a) Path Gain versus Acquisition Order

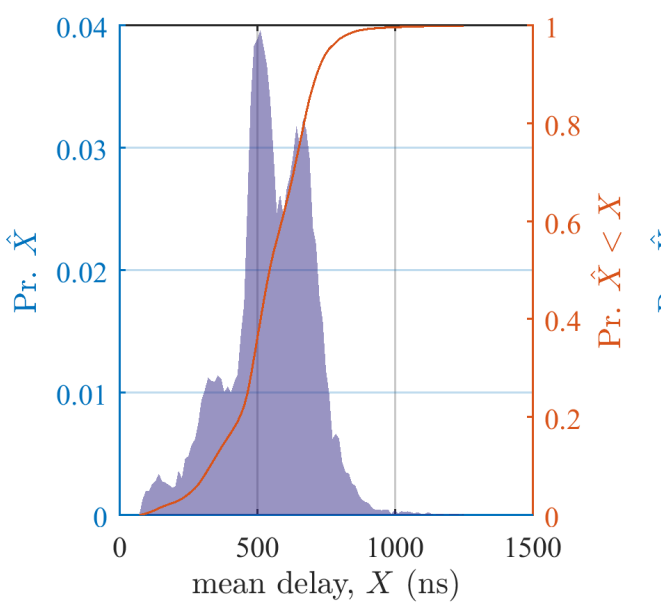

(c) Power Delay

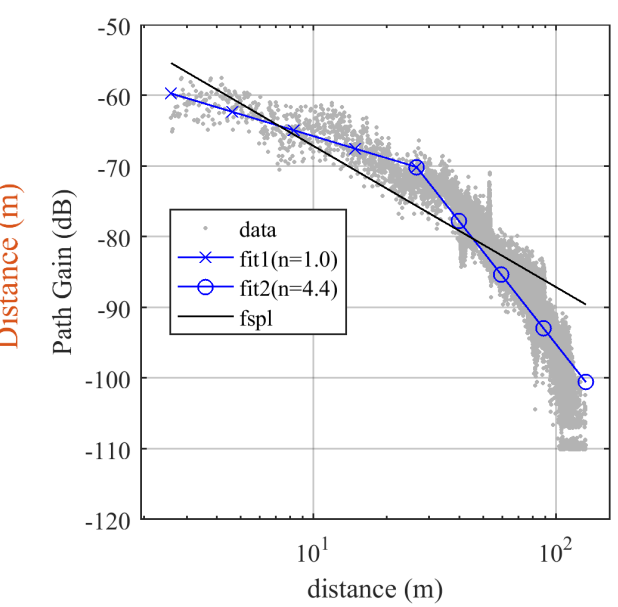

(b) Path Loss versus Distance

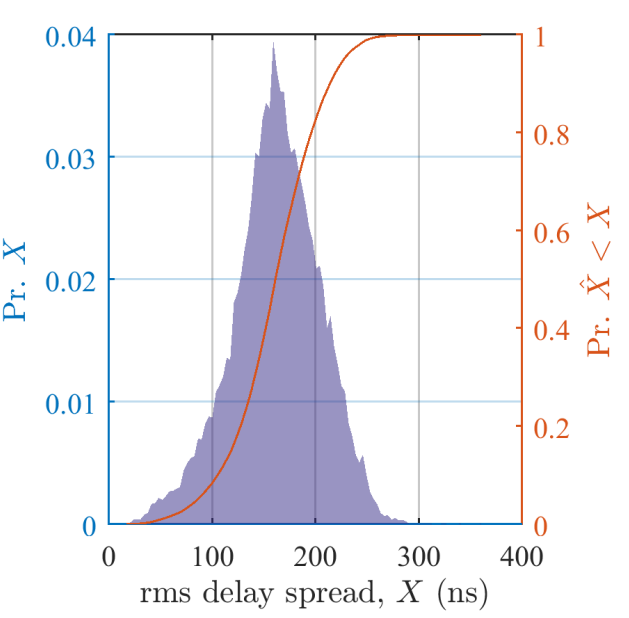

(d) Power Delay Spread

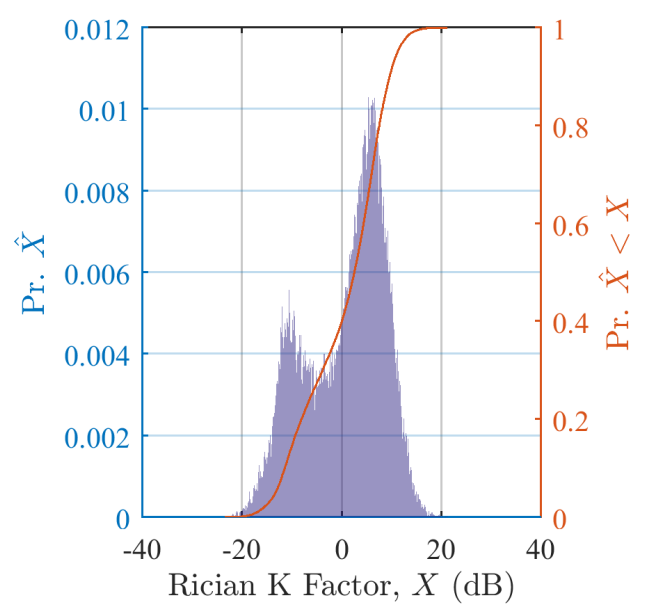

(e) Rician K-factor

Figure A.4.6: Channel characterizations for AAPlantD2 5GHz TX1 hpol internal runB pp channel stats 


\section{A.4.7 AAPlantD2 5GHz TX1 hpol run2 pp.mat}

Table A.21: Measurement Parameters

\begin{tabular}{l|c}
\hline Parameter & Value \\
\hline \hline Mat File & AAPlantD2_5GHz_TX1_hpol_run2_pp.mat \\
Frequency $(\mathrm{GHz})$ & 5.400 \\
Location & AA Plant Day 2 at Automotive Assembly Plant \\
RX Antenna & Omni-directional, Cross Pol \\
RX Antenna Gain & -3.500 \\
TX Antenna & Omni-directional, V Pol \\
TX Antenna Gain & 3.600 \\
TX Power, Watts & 1.250 \\
PN Oversample Factor & 4.000 \\
Sample rate, MHz & 80.000
\end{tabular}

Table A.22: Statistics of Channel Estimates. Outliers are removed using a significance test of 0.95.

\begin{tabular}{l||c|c|c|c||c|c||l}
\hline Estimate & $\min (\cdot)$ & $\operatorname{median}(\cdot)$ & $\operatorname{mean}(\cdot)$ & $\max (\cdot)$ & $\operatorname{std}(\cdot)$ & $\operatorname{mad}(\cdot)$ & outlier info \\
\hline \hline$K(\mathrm{~dB})$, NLOS & -23.4 & 1.5 & 1.4 & 21.1 & 5.8 & 4.6 & N: 29684 \\
\hline$K(\mathrm{~dB})$, LOS & 0.0 & 8.9 & 8.8 & 21.1 & 3.1 & 2.5 & N: 5861 \\
\hline$\tau(\mathrm{ns})$ & 65.8 & 725.8 & 703.0 & 1901.1 & 252.4 & 195.9 & N: 29300 \\
\hline$S(\mathrm{~ns})$ & 0.0 & 180.2 & 179.1 & 424.5 & 51.6 & 41.2 & N: 26226 \\
\hline
\end{tabular}

Table A.23: Statistics of Channel Estimates. Outliers are not removed. N indicates the total number of samples in the population. Outliers are provided at 10 and 20 times $\sigma$.

\begin{tabular}{|c|c|c|c|c|c|c|c|}
\hline Estimate & $\min (\cdot)$ & $\operatorname{median}(\cdot)$ & mean $(\cdot)$ & $\max (\cdot)$ & $\operatorname{std}(\cdot)$ & $\operatorname{mad}(\cdot)$ & outlier info \\
\hline$K(\mathrm{~dB})$, NLOS & -23.4 & 1.5 & 1.4 & 21.1 & 5.8 & 4.6 & $\mathrm{~N}: 29684$ \\
\hline$K(\mathrm{~dB}), \operatorname{LOS}$ & 0.0 & 8.9 & 8.8 & 21.1 & 3.1 & 2.5 & $\mathrm{~N}: 5861$ \\
\hline$\tau(\mathrm{ns})$ & 65.8 & 729.9 & 749.3 & 30451.5 & 590.6 & 239.0 & $\begin{array}{l}\mathrm{N}: 29703 \\
10 \sigma(6655.3): 47 \\
20 \sigma(12561.4): 9\end{array}$ \\
\hline$S(\mathrm{~ns})$ & 0.0 & 189.1 & 652.5 & 33636.5 & 1777.1 & 837.2 & $\begin{array}{l}\mathrm{N}: 29703 \\
10 \sigma(18423.4): 33\end{array}$ \\
\hline
\end{tabular}




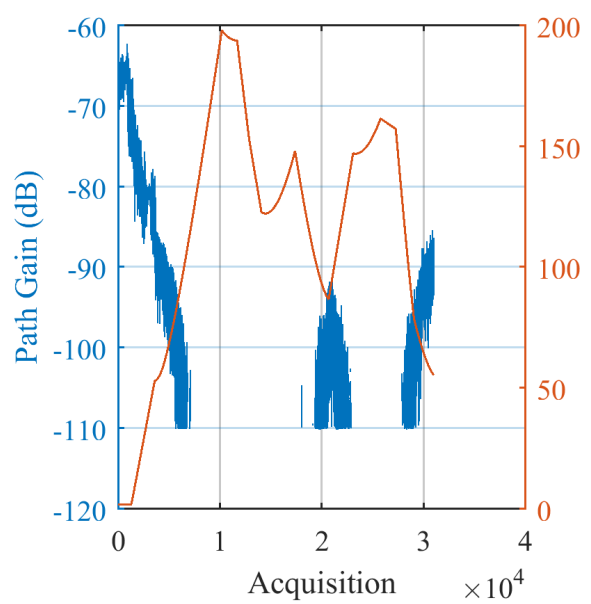

(a) Path Gain versus Acquisition Order

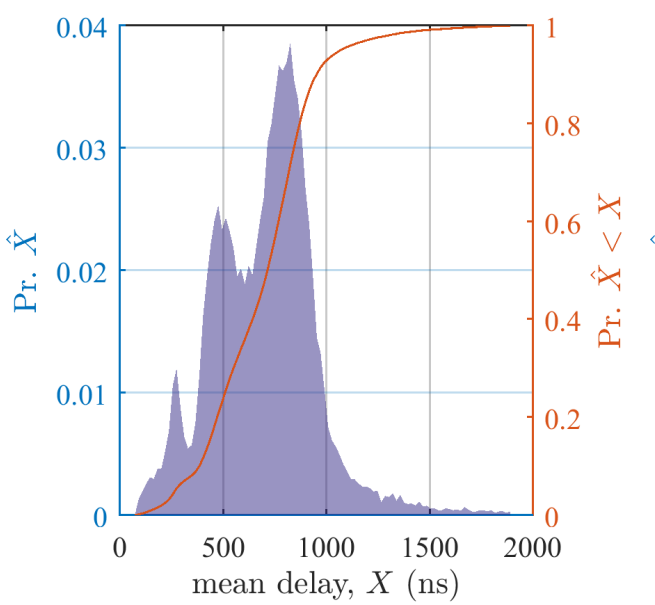

(c) Power Delay

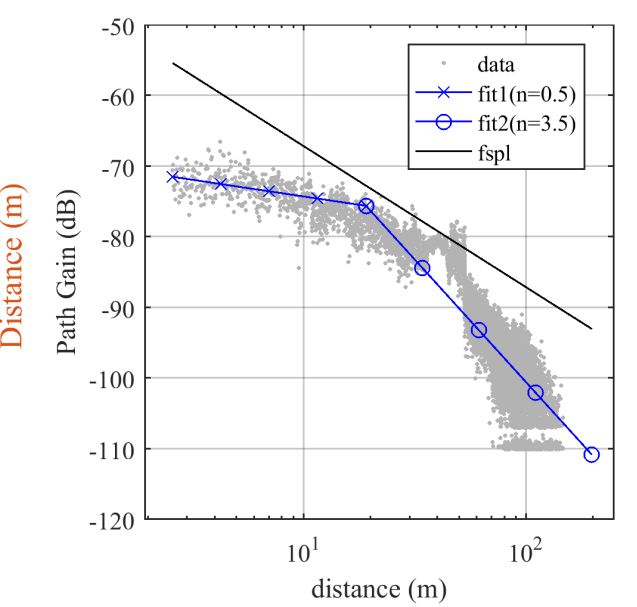

(b) Path Loss versus Distance

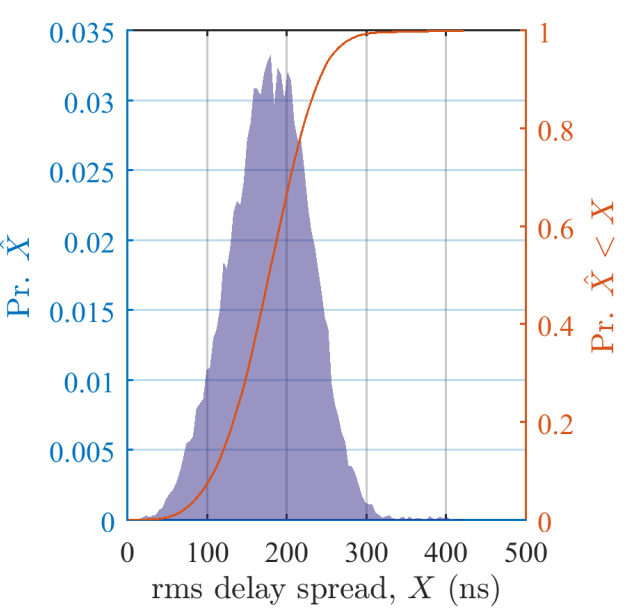

(d) Power Delay Spread

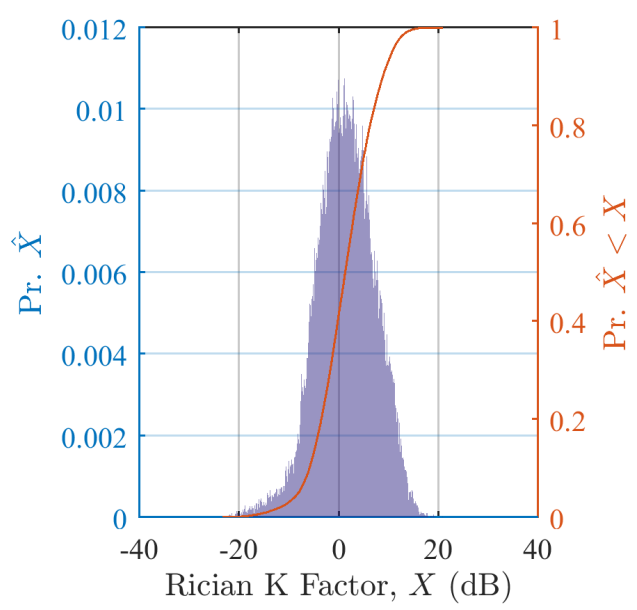

(e) Rician K-factor

Figure A.4.7: Channel characterizations for AAPlantD2 5GHz TX1 hpol run2 pp channel stats 


\section{A.4.8 AAPlantD2 5GHz TX1 vpol internal runA pp.mat}

Table A.24: Measurement Parameters

\begin{tabular}{l|c}
\hline Parameter & Value \\
\hline \hline Mat File & AAPlantD2_5GHz_TX1_vpol_internal_runA_pp.mat \\
Frequency $(\mathrm{GHz})$ & 5.400 \\
Location & AA Plant Day 2 at Automotive Assembly Plant \\
RX Antenna & Omni-directional, V Pol \\
RX Antenna Gain & -3.500 \\
TX Antenna & Omni-directional, V Pol \\
TX Antenna Gain & 3.600 \\
TX Power, Watts & 1.250 \\
PN Oversample Factor & 4.000 \\
Sample rate, MHz & 80.000
\end{tabular}

Table A.25: Statistics of Channel Estimates. Outliers are removed using a significance test of 0.95.

\begin{tabular}{l||c|c|c|c||c|c||l}
\hline Estimate & $\min (\cdot)$ & $\operatorname{median}(\cdot)$ & $\operatorname{mean}(\cdot)$ & $\max (\cdot)$ & $\operatorname{std}(\cdot)$ & $\operatorname{mad}(\cdot)$ & outlier info \\
\hline \hline$K(\mathrm{~dB})$, NLOS & -23.1 & 5.4 & 3.5 & 24.4 & 8.2 & 6.7 & N: 24203 \\
\hline$K(\mathrm{~dB})$, LOS & 4.2 & 11.2 & 11.5 & 23.3 & 2.7 & 2.1 & N: 7335 \\
\hline$\tau(\mathrm{ns})$ & 71.8 & 515.9 & 527.5 & 1077.9 & 151.2 & 118.6 & N: 24203 \\
\hline$S(\mathrm{~ns})$ & 14.8 & 153.5 & 154.4 & 312.4 & 52.8 & 42.1 & N: 24196 \\
\hline
\end{tabular}

Table A.26: Statistics of Channel Estimates. Outliers are not removed. N indicates the total number of samples in the population. Outliers are provided at 10 and 20 times $\sigma$.

\begin{tabular}{l||c|c|c|c||c|c||l}
\hline Estimate & $\min (\cdot)$ & $\operatorname{median}(\cdot)$ & $\operatorname{mean}(\cdot)$ & $\max (\cdot)$ & $\operatorname{std}(\cdot)$ & $\operatorname{mad}(\cdot)$ & outlier info \\
\hline \hline$K(\mathrm{~dB})$, NLOS & -23.1 & 5.4 & 3.5 & 24.4 & 8.2 & 6.7 & $\mathrm{~N}: 24203$ \\
\hline$K(\mathrm{~dB})$, LOS & 4.2 & 11.2 & 11.6 & 24.4 & 2.7 & 2.1 & $\mathrm{~N}: 7337$ \\
\hline$\tau(\mathrm{ns})$ & 71.8 & 515.9 & 527.5 & 1077.9 & 151.2 & 118.6 & $\mathrm{~N}: 24203$ \\
\hline$S(\mathrm{~ns})$ & 14.8 & 153.5 & 155.0 & 4017.0 & 67.8 & 42.8 & $\mathrm{~N}: 24203$ \\
& & & & & & & $10 \sigma(832.9): 6$ \\
& & & & & & & $20 \sigma(1510.7): 5$ \\
\hline
\end{tabular}




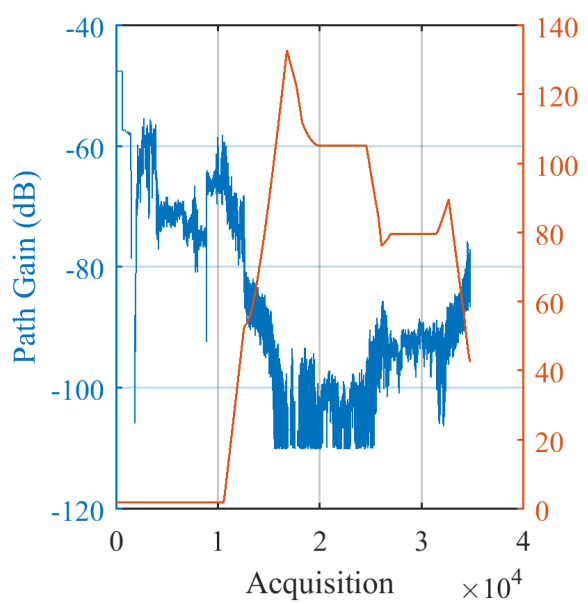

(a) Path Gain versus Acquisition Order

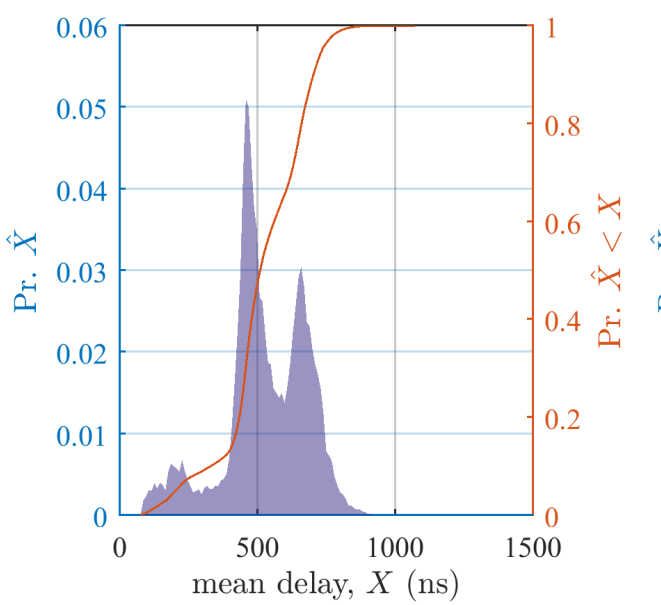

(c) Power Delay

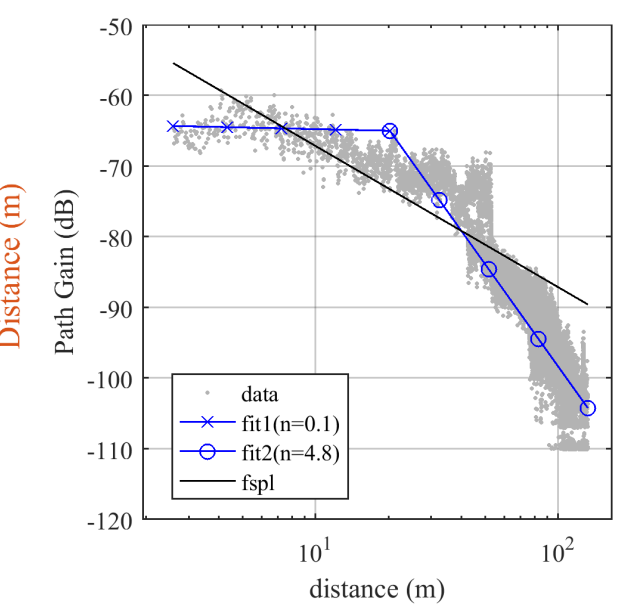

(b) Path Loss versus Distance

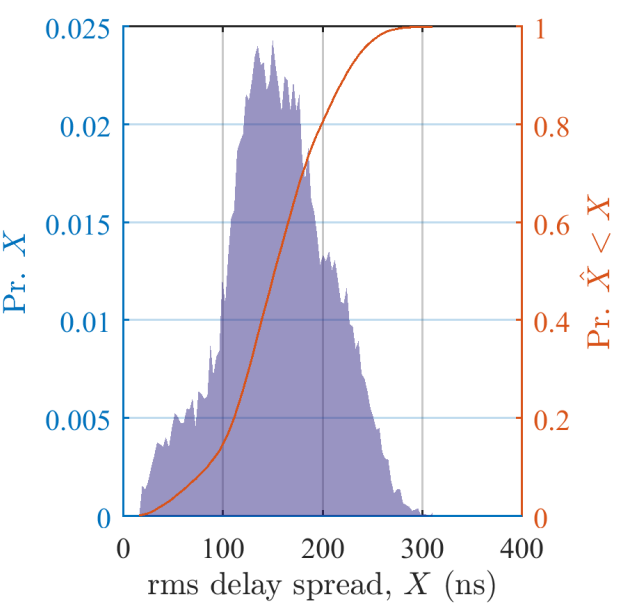

(d) Power Delay Spread

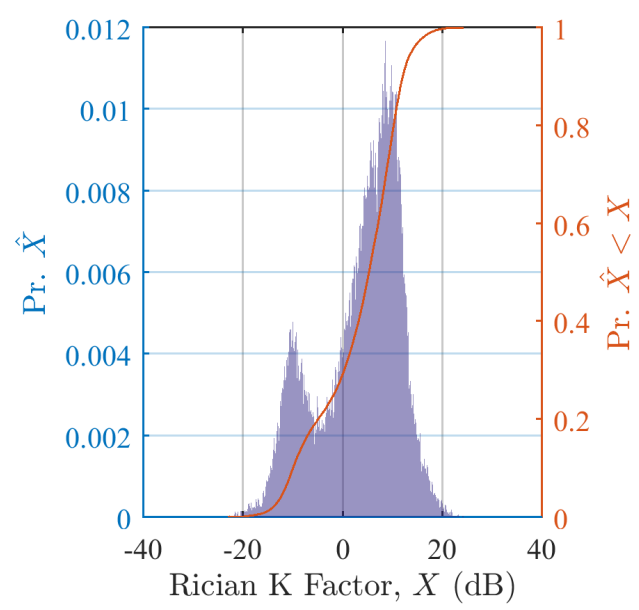

(e) Rician K-factor

Figure A.4.8: Channel characterizations for AAPlantD2 5GHz TX1 vpol internal runA pp channel stats 


\section{A.4.9 AAPlantD2 5GHz TX1 vpol run1 pp.mat}

Table A.27: Measurement Parameters

\begin{tabular}{l|c}
\hline Parameter & Value \\
\hline \hline Mat File & AAPlantD2_5GHz_TX1_vpol_run1_pp.mat \\
Frequency $(\mathrm{GHz})$ & 5.400 \\
Location & AA Plant Day 2 at Automotive Assembly Plant \\
RX Antenna & Omni-directional, V Pol \\
RX Antenna Gain & -3.500 \\
TX Antenna & Omni-directional, V Pol \\
TX Antenna Gain & 3.600 \\
TX Power, Watts & 1.250 \\
PN Oversample Factor & 4.000 \\
Sample rate, MHz & 80.000
\end{tabular}

Table A.28: Statistics of Channel Estimates. Outliers are removed using a significance test of 0.95.

\begin{tabular}{l||c|c|c|c||c|c||l}
\hline Estimate & $\min (\cdot)$ & $\operatorname{median}(\cdot)$ & $\operatorname{mean}(\cdot)$ & $\max (\cdot)$ & $\operatorname{std}(\cdot)$ & $\operatorname{mad}(\cdot)$ & outlier info \\
\hline \hline$K(\mathrm{~dB})$, NLOS & -23.5 & 5.4 & 4.7 & 24.2 & 7.1 & 5.6 & N: 29261 \\
\hline$K(\mathrm{~dB})$, LOS & 1.1 & 11.3 & 11.6 & 24.2 & 3.1 & 2.5 & N: 10261 \\
\hline$\tau(\mathrm{ns})$ & 38.0 & 664.8 & 644.4 & 1702.2 & 221.4 & 178.0 & N: 29076 \\
\hline$S(\mathrm{~ns})$ & 14.4 & 182.0 & 177.4 & 420.4 & 57.6 & 46.0 & N: 28717 \\
\hline
\end{tabular}

Table A.29: Statistics of Channel Estimates. Outliers are not removed. N indicates the total number of samples in the population. Outliers are provided at 10 and 20 times $\sigma$.

\begin{tabular}{l||c|c|c|c||c|c||l}
\hline Estimate & $\min (\cdot)$ & $\operatorname{median}(\cdot)$ & $\operatorname{mean}(\cdot)$ & $\max (\cdot)$ & $\operatorname{std}(\cdot)$ & $\operatorname{mad}(\cdot)$ & outlier info \\
\hline \hline$K(\mathrm{~dB})$, NLOS & -23.5 & 5.4 & 4.7 & 24.2 & 7.1 & 5.6 & $\mathrm{~N}: 29261$ \\
\hline$K(\mathrm{~dB})$, LOS & 1.1 & 11.3 & 11.6 & 24.2 & 3.1 & 2.5 & $\mathrm{~N}: 10261$ \\
\hline$\tau(\mathrm{ns})$ & 38.0 & 667.0 & 659.6 & 13331.6 & 315.1 & 191.3 & $\mathrm{~N}: 29261$ \\
& & & & & & & $10 \sigma(3810.6): 32$ \\
& & & & & & & $20 \sigma(6961.7): 4$ \\
\hline$S(\mathrm{~ns})$ & 14.4 & 183.4 & 266.2 & 28844.6 & 845.8 & 176.0 & $\mathrm{~N}: 29261$ \\
& & & & & & & $10 \sigma(8723.9): 94$ \\
& & & & & & & $20 \sigma(17181.5): 5$ \\
\hline
\end{tabular}




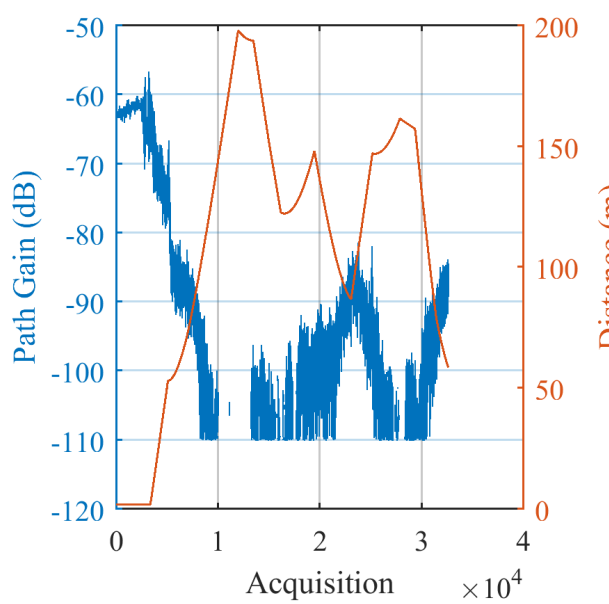

(a) Path Gain versus Acquisition Order

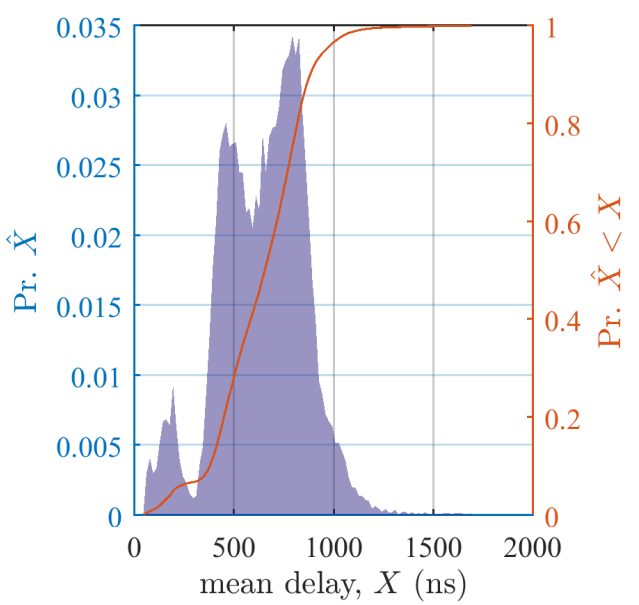

(c) Power Delay

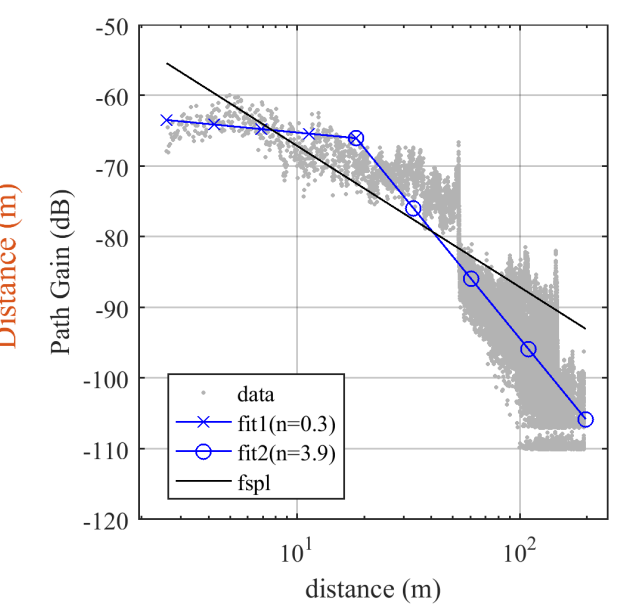

(b) Path Loss versus Distance

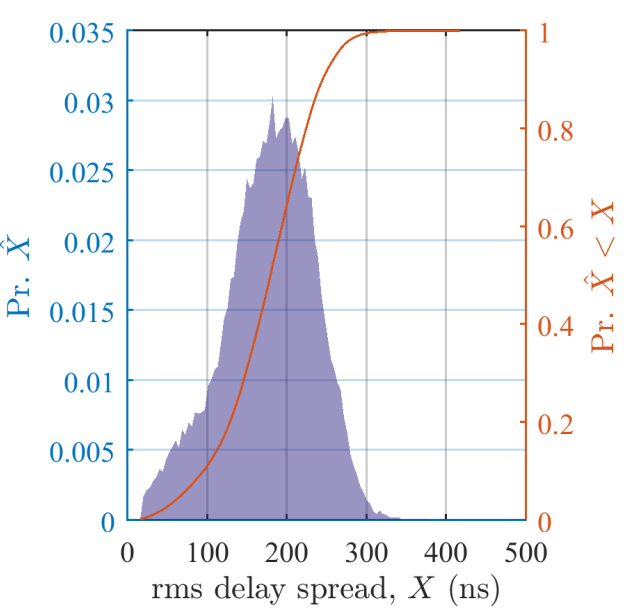

(d) Power Delay Spread

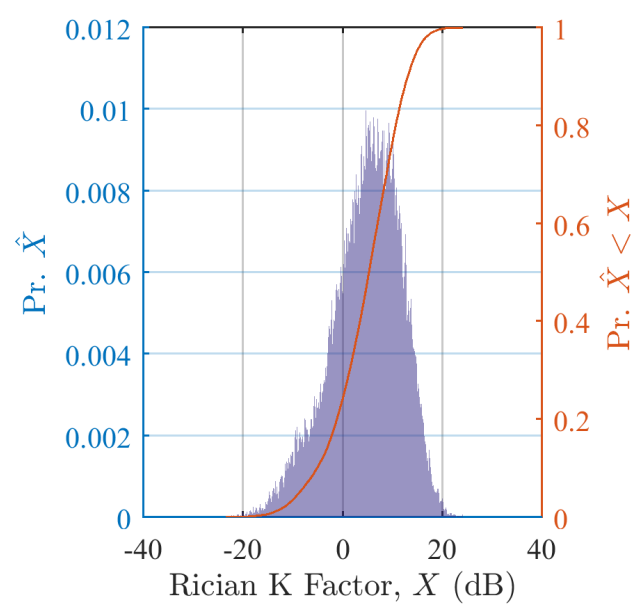

(e) Rician K-factor

Figure A.4.9: Channel characterizations for AAPlantD2 5GHz TX1 vpol run1 pp channel stats 


\section{A.4.10 AAPlantD3 2GHz TX2b hpol internal run36 pp.mat}

Table A.30: Measurement Parameters

\begin{tabular}{l|c}
\hline Parameter & Value \\
\hline \hline Mat File & AAPlantD3_2GHz_TX2b_hpol_internal_run36_pp.mat \\
Frequency (GHz) & 2.245 \\
Location & AA Plant Day 3 at Automotive Assembly Plant \\
RX Antenna & Omni-directional, Cross Pol \\
RX Antenna Gain & -4.200 \\
TX Antenna & Omni-directional, V Pol \\
TX Antenna Gain & 2.900 \\
TX Power, Watts & 1.500 \\
PN Oversample Factor & 4.000 \\
Sample rate, MHz & 80.000
\end{tabular}

Table A.31: Statistics of Channel Estimates. Outliers are removed using a significance test of 0.95.

\begin{tabular}{l||c|c|c|c||c|c||l}
\hline Estimate & $\min (\cdot)$ & $\operatorname{median}(\cdot)$ & $\operatorname{mean}(\cdot)$ & $\max (\cdot)$ & $\operatorname{std}(\cdot)$ & $\operatorname{mad}(\cdot)$ & outlier info \\
\hline \hline$K(\mathrm{~dB})$, NLOS & -23.3 & 1.8 & 1.5 & 18.7 & 5.5 & 4.2 & N: 30081 \\
\hline$K(\mathrm{~dB})$, LOS & 0.0 & 7.9 & 7.6 & 18.7 & 3.7 & 3.0 & N: 5636 \\
\hline$\tau(\mathrm{ns})$ & 35.3 & 485.7 & 492.1 & 1374.9 & 184.5 & 139.0 & N: 29864 \\
\hline$S(\mathrm{~ns})$ & 0.0 & 141.4 & 135.1 & 336.9 & 42.2 & 31.9 & N: 29401 \\
\hline
\end{tabular}

Table A.32: Statistics of Channel Estimates. Outliers are not removed. N indicates the total number of samples in the population. Outliers are provided at 10 and 20 times $\sigma$.

\begin{tabular}{|c|c|c|c|c|c|c|c|}
\hline Estimate & $\min (\cdot)$ & $\operatorname{median}(\cdot)$ & mean $(\cdot)$ & $\max (\cdot)$ & $\operatorname{std}(\cdot)$ & $\operatorname{mad}(\cdot)$ & outlier info \\
\hline$K(\mathrm{~dB}), \mathrm{NLOS}$ & -23.3 & 1.8 & 1.5 & 18.7 & 5.5 & 4.2 & $\mathrm{~N}: 30081$ \\
\hline$K(\mathrm{~dB}), \mathrm{LOS}$ & 0.0 & 7.9 & 7.6 & 18.7 & 3.7 & 3.0 & $\mathrm{~N}: 5636$ \\
\hline$\tau(\mathrm{ns})$ & 35.3 & 489.1 & 625.2 & 81425.0 & 1887.6 & 309.9 & $\begin{array}{l}\mathrm{N}: 30455 \\
10 \sigma(19501.4): 43 \\
20 \sigma(38377.5): 23\end{array}$ \\
\hline$S(\mathrm{~ns})$ & 0.0 & 142.9 & 405.1 & 39006.1 & 1884.9 & 521.3 & $\begin{array}{l}\mathrm{N}: 30455 \\
10 \sigma(19254.3): 73 \\
20 \sigma(38103.6): 1\end{array}$ \\
\hline
\end{tabular}




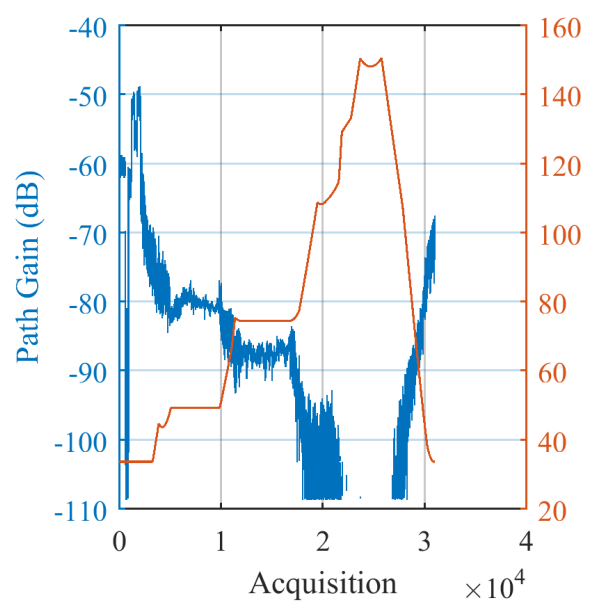

(a) Path Gain versus Acquisition Order

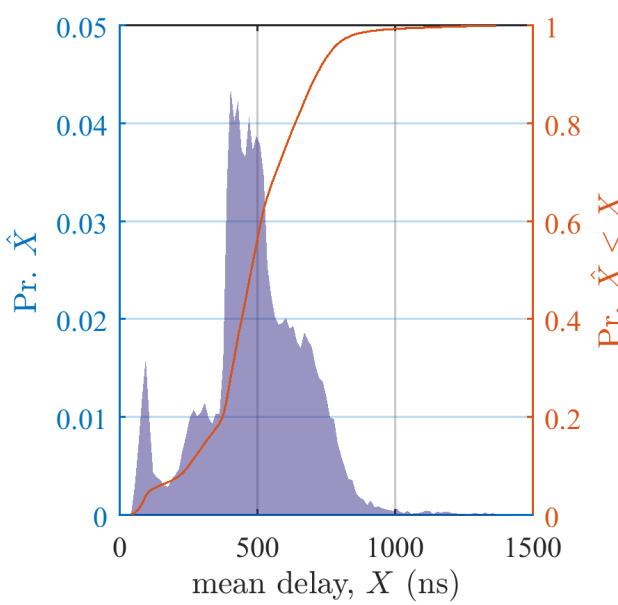

(c) Power Delay

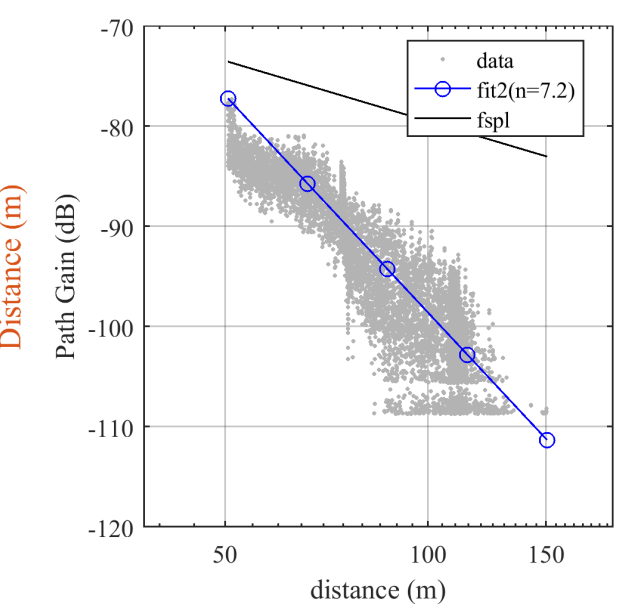

(b) Path Loss versus Distance

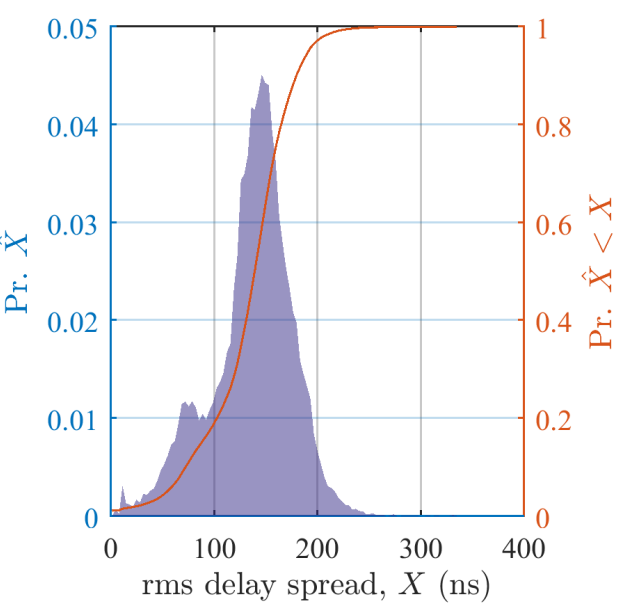

(d) Power Delay Spread

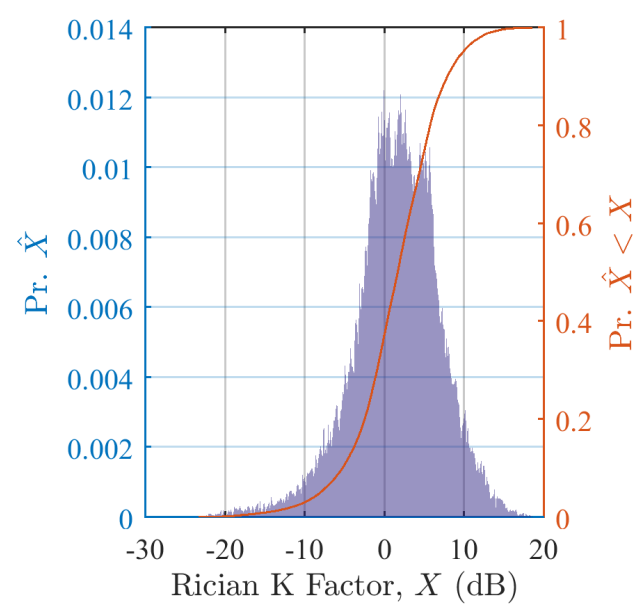

(e) Rician K-factor

Figure A.4.10: Channel characterizations for AAPlantD3 2GHz TX2b hpol internal run36 pp channel stats 


\section{A.4.11 AAPlantD3 2GHz TX2b vpol internal run33 pp.mat}

Table A.33: Measurement Parameters

\begin{tabular}{l|c}
\hline Parameter & Value \\
\hline \hline Mat File & AAPlantD3_2GHz_TX2b_vpol_interna \\
Frequency (GHz) & 2.245 \\
Location & AA Plant Day 3 at Automotive Ass \\
RX Antenna & Omni-directional, V Pol \\
RX Antenna Gain & -4.200 \\
TX Antenna & Omni-directional, V Pol \\
TX Antenna Gain & 2.900 \\
TX Power, Watts & 1.500 \\
PN Oversample Factor & 4.000 \\
Sample rate, MHz & 80.000
\end{tabular}

Table A.34: Statistics of Channel Estimates. Outliers are removed using a significance test of 0.95.

\begin{tabular}{l||c|c|c|c||c|c||l}
\hline Estimate & $\min (\cdot)$ & $\operatorname{median}(\cdot)$ & $\operatorname{mean}(\cdot)$ & $\max (\cdot)$ & $\operatorname{std}(\cdot)$ & $\operatorname{mad}(\cdot)$ & outlier info \\
\hline \hline$K(\mathrm{~dB})$, NLOS & -23.6 & 4.8 & 4.7 & 25.1 & 6.8 & 5.4 & N: 35788 \\
\hline$K(\mathrm{~dB})$, LOS & 0.0 & 11.0 & 11.4 & 25.1 & 4.1 & 3.1 & N: 12712 \\
\hline$\tau(\mathrm{ns})$ & 0.0 & 390.3 & 391.3 & 1552.0 & 242.3 & 188.7 & N: 36098 \\
\hline$S(\mathrm{~ns})$ & 0.0 & 121.9 & 111.9 & 372.5 & 55.5 & 45.8 & N: 35996 \\
\hline
\end{tabular}

Table A.35: Statistics of Channel Estimates. Outliers are not removed. N indicates the total number of samples in the population. Outliers are provided at 10 and 20 times $\sigma$.

\begin{tabular}{|c|c|c|c|c|c|c|c|}
\hline Estimate & $\min (\cdot)$ & $\operatorname{median}(\cdot)$ & mean $(\cdot)$ & $\max (\cdot)$ & $\operatorname{std}(\cdot)$ & $\operatorname{mad}(\cdot)$ & outlier info \\
\hline$K(\mathrm{~dB})$, NLOS & -23.6 & 4.8 & 4.7 & 25.1 & 6.8 & 5.4 & $\mathrm{~N}: 35788$ \\
\hline$K(\mathrm{~dB}), \operatorname{LOS}$ & 0.0 & 11.0 & 11.4 & 25.1 & 4.1 & 3.1 & $\mathrm{~N}: 12712$ \\
\hline$\tau(\mathrm{ns})$ & 0.0 & 402.8 & 1582.3 & 81825.0 & 7689.2 & 2285.8 & $\begin{array}{l}\mathrm{N}: 37617 \\
10 \sigma(78474.8): 31\end{array}$ \\
\hline$S(\mathrm{~ns})$ & 0.0 & 124.3 & 381.4 & 36383.3 & 1704.2 & 515.8 & $\begin{array}{l}\mathrm{N}: 37617 \\
10 \sigma(17423.9): 78 \\
20 \sigma(34466.3): 1\end{array}$ \\
\hline
\end{tabular}




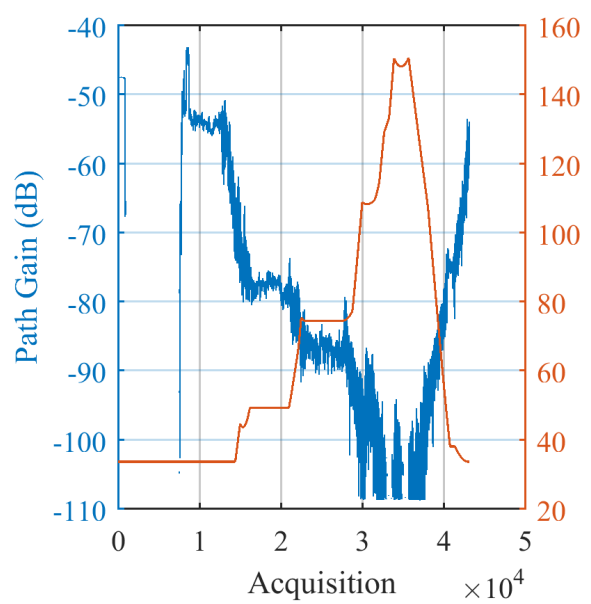

(a) Path Gain versus Acquisition Order

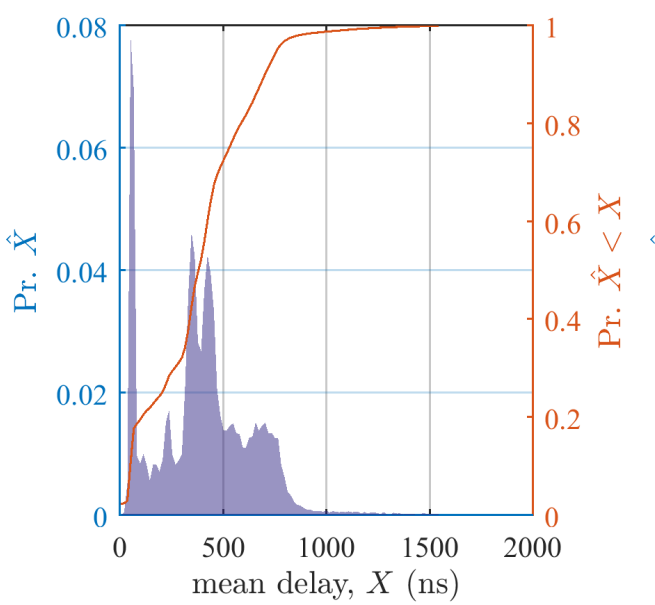

(c) Power Delay

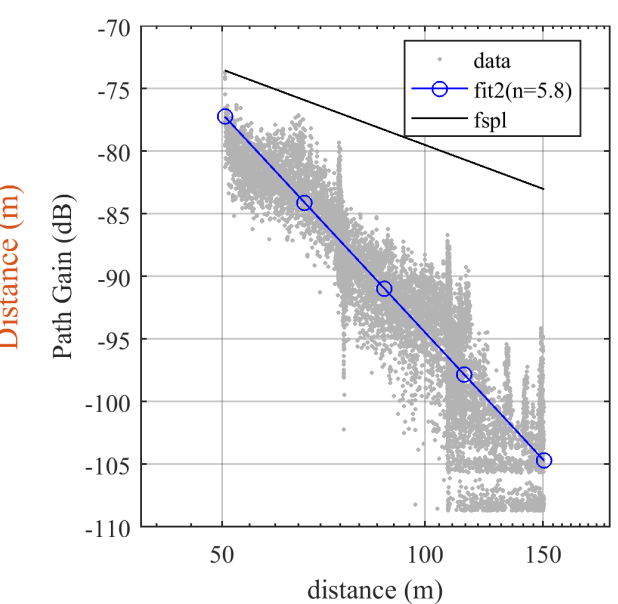

(b) Path Loss versus Distance

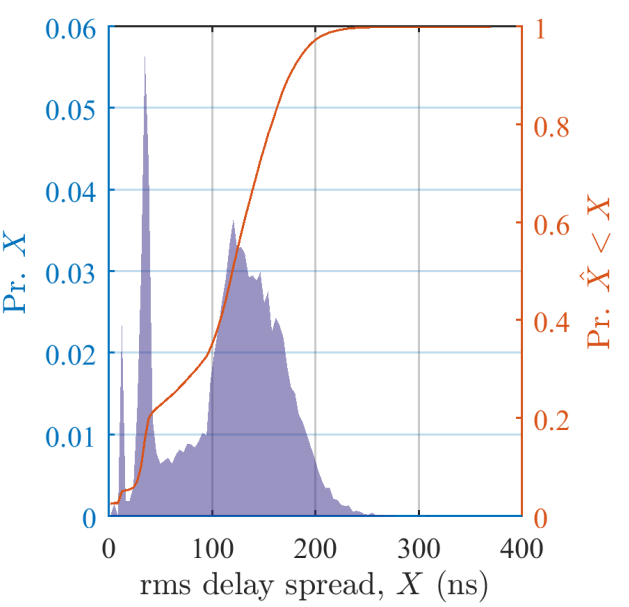

(d) Power Delay Spread

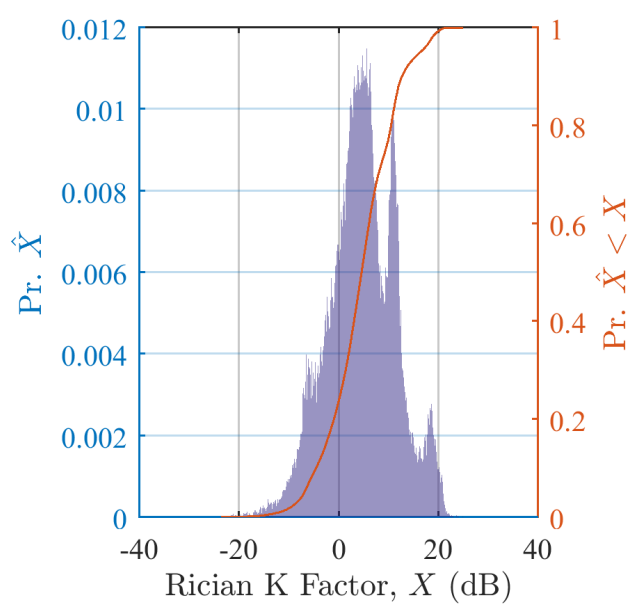

(e) Rician K-factor

Figure A.4.11: Channel characterizations for AAPlantD3 2GHz TX2b vpol internal run33 pp channel stats 


\section{A.4.12 AAPlantD3 2GHz TX2b vpol run32 pp.mat}

Table A.36: Measurement Parameters

\begin{tabular}{l|c}
\hline Parameter & Value \\
\hline \hline Mat File & AAPlantD3_2GHz_TX2b_vpol_run32_pp.mat \\
Frequency $(\mathrm{GHz})$ & 2.245 \\
Location & AA Plant Day 3 at Automotive Assembly Plant \\
RX Antenna & Omni-directional, V Pol \\
RX Antenna Gain & -4.200 \\
TX Antenna & Omni-directional, V Pol \\
TX Antenna Gain & 2.900 \\
TX Power, Watts & 1.500 \\
PN Oversample Factor & 4.000 \\
Sample rate, MHz & 80.000
\end{tabular}

Table A.37: Statistics of Channel Estimates. Outliers are removed using a significance test of 0.95.

\begin{tabular}{l||c|c|c|c||c|c||l}
\hline Estimate & $\min (\cdot)$ & $\operatorname{median}(\cdot)$ & $\operatorname{mean}(\cdot)$ & $\max (\cdot)$ & $\operatorname{std}(\cdot)$ & $\operatorname{mad}(\cdot)$ & outlier info \\
\hline \hline$K(\mathrm{~dB})$, NLOS & -24.3 & 2.5 & 4.2 & 26.6 & 7.3 & 6.0 & N: 36474 \\
\hline$K(\mathrm{~dB})$, LOS & 0.0 & 11.0 & 10.9 & 26.6 & 5.7 & 4.7 & N: 14687 \\
\hline$\tau(\mathrm{ns})$ & 19.3 & 674.4 & 642.3 & 2589.6 & 403.9 & 316.7 & N: 35751 \\
\hline$S(\mathrm{~ns})$ & 0.0 & 127.8 & 121.7 & 393.2 & 56.7 & 46.0 & N: 33903 \\
\hline
\end{tabular}

Table A.38: Statistics of Channel Estimates. Outliers are not removed. N indicates the total number of samples in the population. Outliers are provided at 10 and 20 times $\sigma$.

\begin{tabular}{|c|c|c|c|c|c|c|c|}
\hline Estimate & $\min (\cdot)$ & $\operatorname{median}(\cdot)$ & mean $(\cdot)$ & $\max (\cdot)$ & $\operatorname{std}(\cdot)$ & $\operatorname{mad}(\cdot)$ & outlier info \\
\hline$K(\mathrm{~dB})$, NLOS & -24.3 & 2.5 & 4.2 & 26.6 & 7.3 & 6.0 & $\mathrm{~N}: 36474$ \\
\hline$K(\mathrm{~dB}), \operatorname{LOS}$ & 0.0 & 11.0 & 10.9 & 26.6 & 5.7 & 4.7 & $\mathrm{~N}: 14687$ \\
\hline$\tau(\mathrm{ns})$ & 19.3 & 686.8 & 942.1 & 81475.0 & 2820.3 & 675.6 & $\begin{array}{l}\mathrm{N}: 37003 \\
10 \sigma(29145.3): 86 \\
20 \sigma(57348.5): 26\end{array}$ \\
\hline$S(\mathrm{~ns})$ & 0.0 & 134.0 & 790.6 & 39159.3 & 2917.6 & 1226.9 & $\begin{array}{l}\mathrm{N}: 37003 \\
10 \sigma(29967.0): 34\end{array}$ \\
\hline
\end{tabular}




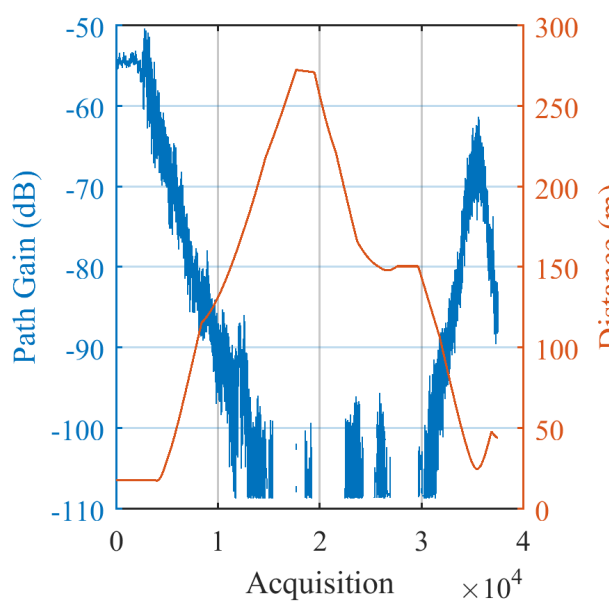

(a) Path Gain versus Acquisition Order

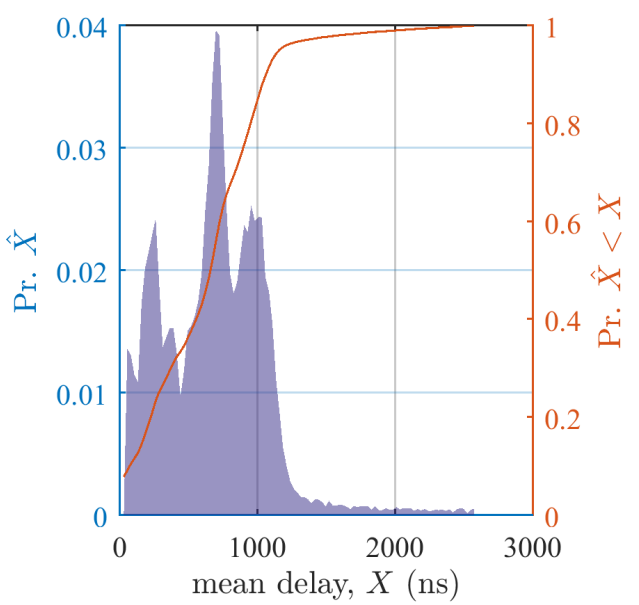

(c) Power Delay

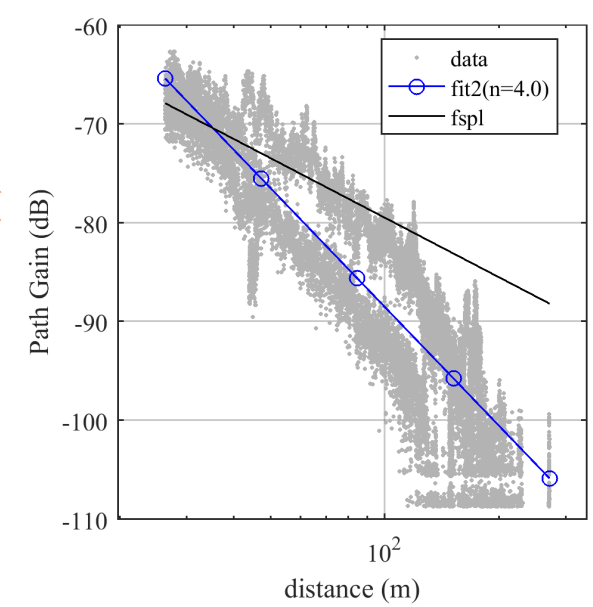

(b) Path Loss versus Distance

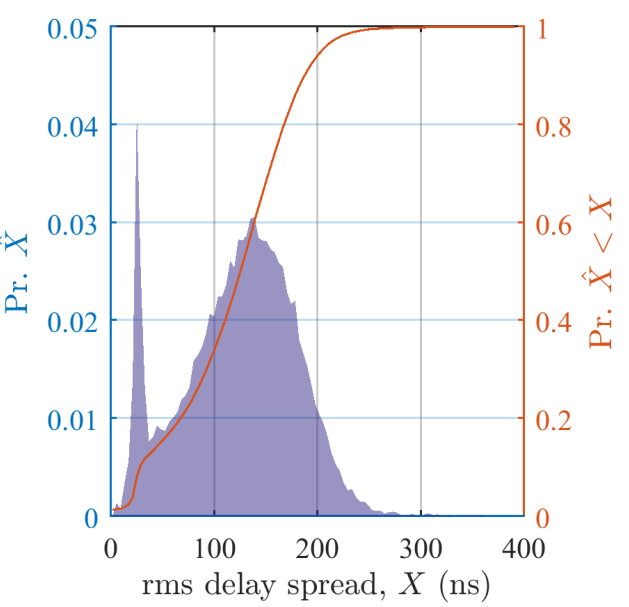

(d) Power Delay Spread

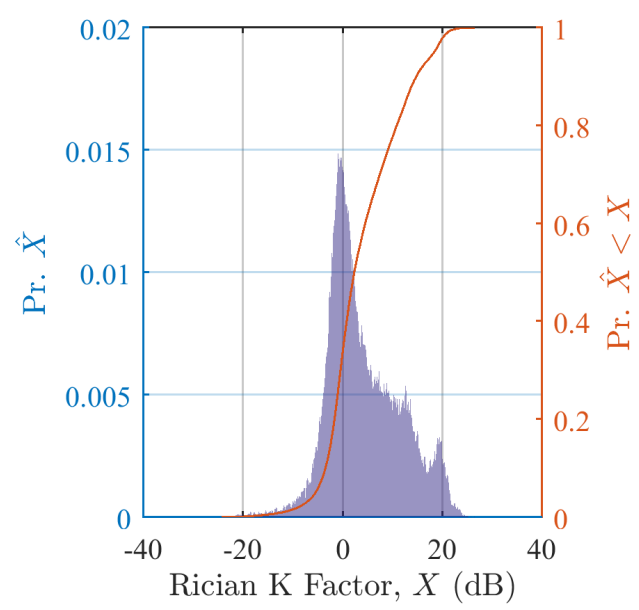

(e) Rician K-factor

Figure A.4.12: Channel characterizations for AAPlantD3 2GHz TX2b vpol run32 pp channel stats 


\section{A.4.13 AAPlantD3 2GHz Tx2b hpol run34 pp.mat}

Table A.39: Measurement Parameters

\begin{tabular}{l|c}
\hline Parameter & Value \\
\hline \hline Mat File & AAPlantD3_2GHz_Tx2b_hpol_run34_pp.mat \\
Frequency $(\mathrm{GHz})$ & 2.245 \\
Location & AA Plant Day 3 at Automotive Assembly Plant \\
RX Antenna & Omni-directional, Cross Pol \\
RX Antenna Gain & -4.200 \\
TX Antenna & Omni-directional, V Pol \\
TX Antenna Gain & 2.900 \\
TX Power, Watts & 1.500 \\
PN Oversample Factor & 4.000 \\
Sample rate, MHz & 80.000
\end{tabular}

Table A.40: Statistics of Channel Estimates. Outliers are removed using a significance test of 0.95.

\begin{tabular}{l||c|c|c|c||c|c||l}
\hline Estimate & $\min (\cdot)$ & $\operatorname{median}(\cdot)$ & $\operatorname{mean}(\cdot)$ & $\max (\cdot)$ & $\operatorname{std}(\cdot)$ & $\operatorname{mad}(\cdot)$ & outlier info \\
\hline \hline$K(\mathrm{~dB})$, NLOS & -23.0 & 0.7 & 1.9 & 21.1 & 6.1 & 4.6 & N: 24606 \\
\hline$K(\mathrm{~dB})$, LOS & 0.0 & 8.4 & 8.0 & 21.1 & 5.4 & 4.5 & N: 6770 \\
\hline$\tau(\mathrm{ns})$ & 4.9 & 556.6 & 538.6 & 1973.6 & 303.6 & 254.6 & N: 25487 \\
\hline$S(\mathrm{~ns})$ & 0.0 & 120.9 & 114.2 & 397.6 & 59.8 & 48.2 & N: 25454 \\
\hline
\end{tabular}

Table A.41: Statistics of Channel Estimates. Outliers are not removed. N indicates the total number of samples in the population. Outliers are provided at 10 and 20 times $\sigma$.

\begin{tabular}{l||c|c|c|c||c|c||l}
\hline Estimate & $\min (\cdot)$ & $\operatorname{median}(\cdot)$ & $\operatorname{mean}(\cdot)$ & $\max (\cdot)$ & $\operatorname{std}(\cdot)$ & $\operatorname{mad}(\cdot)$ & outlier info \\
\hline \hline$K(\mathrm{~dB})$, NLOS & -23.0 & 0.7 & 1.9 & 21.1 & 6.1 & 4.6 & $\mathrm{~N}: 24606$ \\
\hline$K(\mathrm{~dB})$, LOS & 0.0 & 8.4 & 8.0 & 21.1 & 5.4 & 4.5 & $\mathrm{~N}: 6770$ \\
\hline$\tau(\mathrm{ns})$ & 4.9 & 579.0 & 1528.2 & 81812.5 & 6507.0 & 1888.6 & $\begin{array}{l}\mathrm{N}: 26731 \\
10 \sigma(66598.3): 79\end{array}$ \\
\hline$S(\mathrm{~ns})$ & & & & & & & \\
& 0.0 & 124.7 & 638.2 & 40299.3 & 3061.8 & 998.2 & $\begin{array}{l}\mathrm{N}: 26731 \\
10 \sigma(31256.3): 47\end{array}$ \\
\hline
\end{tabular}




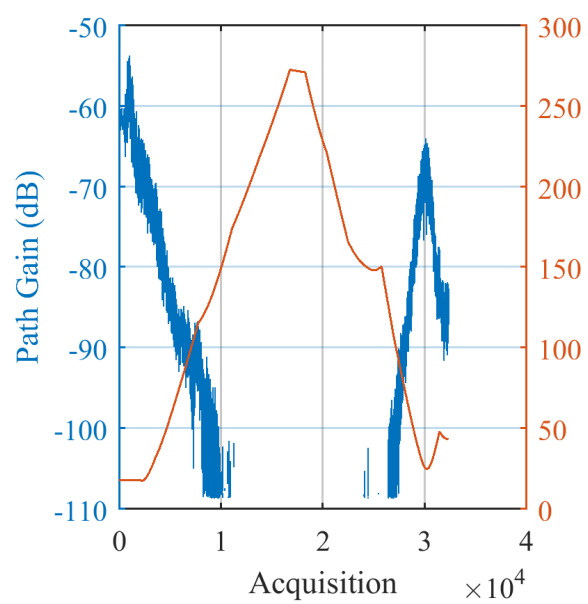

(a) Path Gain versus Acquisition Order

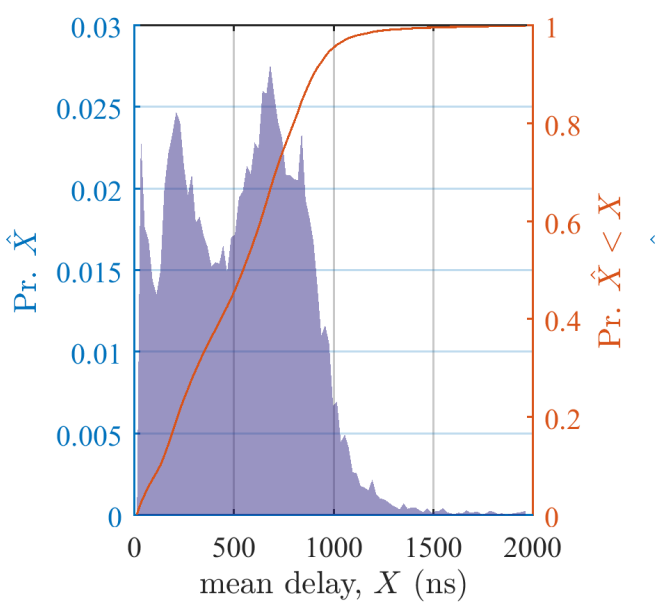

(c) Power Delay

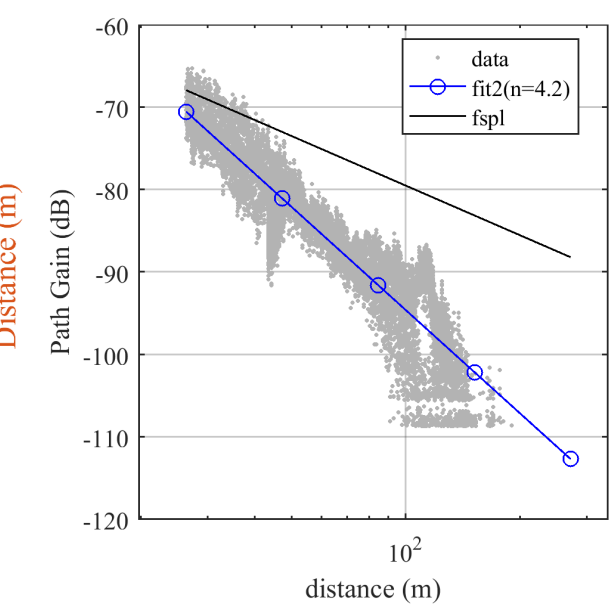

(b) Path Loss versus Distance

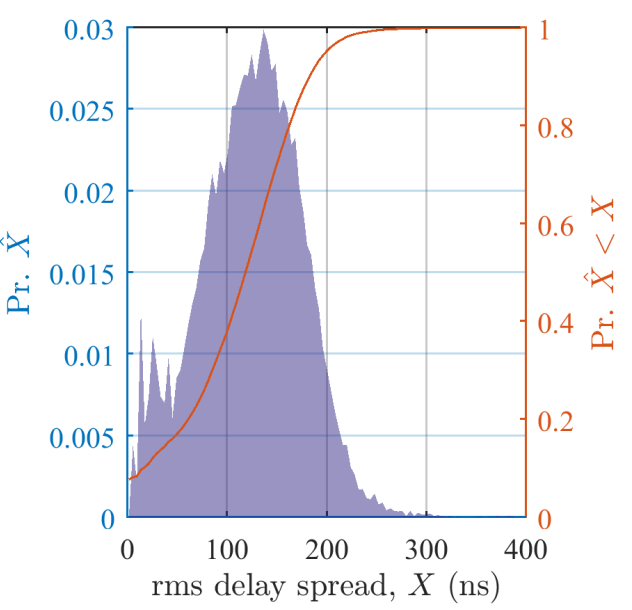

(d) Power Delay Spread

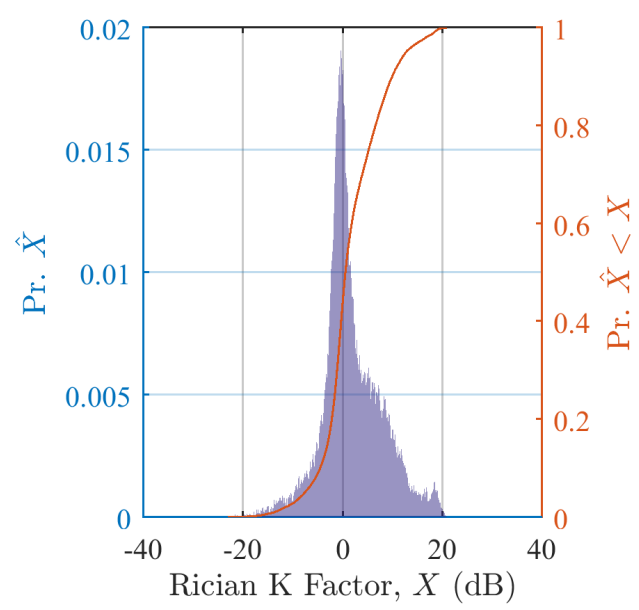

(e) Rician K-factor

Figure A.4.13: Channel characterizations for AAPlantD3 2GHz Tx2b hpol run34 pp channel stats 


\section{A.4.14 AAPlantD3 5GHz TX2b hpol internal run39 pp.mat}

Table A.42: Measurement Parameters

\begin{tabular}{l|c}
\hline Parameter & Value \\
\hline \hline Mat File & AAPlantD3_5GHz_TX2b_hpol_internal_run39_pp.mat \\
Frequency (GHz) & 5.400 \\
Location & AA Plant Day 3 at Automotive Assembly Plant \\
RX Antenna & Omni-directional, Cross Pol \\
RX Antenna Gain & -3.500 \\
TX Antenna & Omni-directional, V Pol \\
TX Antenna Gain & 3.600 \\
TX Power, Watts & 1.250 \\
PN Oversample Factor & 4.000 \\
Sample rate, MHz & 80.000
\end{tabular}

Table A.43: Statistics of Channel Estimates. Outliers are removed using a significance test of 0.95.

\begin{tabular}{l||c|c|c|c||c|c||l}
\hline Estimate & $\min (\cdot)$ & $\operatorname{median}(\cdot)$ & $\operatorname{mean}(\cdot)$ & $\max (\cdot)$ & $\operatorname{std}(\cdot)$ & $\operatorname{mad}(\cdot)$ & outlier info \\
\hline \hline$K(\mathrm{~dB})$, NLOS & -23.2 & 4.0 & 3.0 & 25.8 & 8.4 & 6.9 & N: 29430 \\
\hline$K(\mathrm{~dB})$, LOS & 2.3 & 11.3 & 11.9 & 25.8 & 3.7 & 3.1 & N: 9024 \\
\hline$\tau(\mathrm{ns})$ & 39.9 & 453.1 & 471.7 & 1716.8 & 263.6 & 206.1 & N: 29397 \\
\hline$S(\mathrm{~ns})$ & 14.2 & 146.1 & 146.0 & 451.9 & 64.7 & 51.4 & N: 28474 \\
\hline
\end{tabular}

Table A.44: Statistics of Channel Estimates. Outliers are not removed. N indicates the total number of samples in the population. Outliers are provided at 10 and 20 times $\sigma$.

\begin{tabular}{l||c|c|c|c||c|c||l}
\hline Estimate & $\min (\cdot)$ & $\operatorname{median}(\cdot)$ & $\operatorname{mean}(\cdot)$ & $\max (\cdot)$ & $\operatorname{std}(\cdot)$ & $\operatorname{mad}(\cdot)$ & outlier info \\
\hline \hline$K(\mathrm{~dB})$, NLOS & -23.2 & 4.0 & 3.0 & 25.8 & 8.4 & 6.9 & N: 29430 \\
\hline$K(\mathrm{~dB})$, LOS & 2.3 & 11.3 & 11.9 & 25.8 & 3.7 & 3.1 & N: 9024 \\
\hline$\tau(\mathrm{ns})$ & 39.9 & 453.3 & 473.4 & 3058.1 & 268.7 & 207.8 & N: 29430 \\
\hline$S(\mathrm{~ns})$ & 14.2 & 148.3 & 244.5 & 11410.9 & 632.4 & 193.3 & $\mathrm{~N}: 29430$ \\
& & & & & & & $10 \sigma(6568.9): 49$ \\
\hline
\end{tabular}




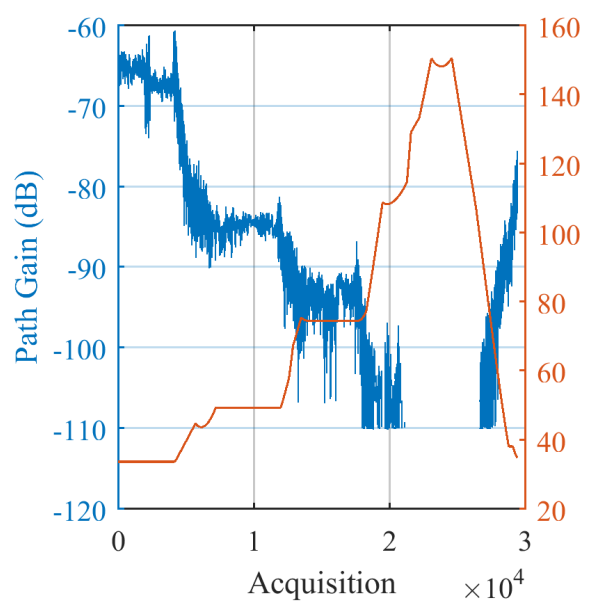

(a) Path Gain versus Acquisition Order

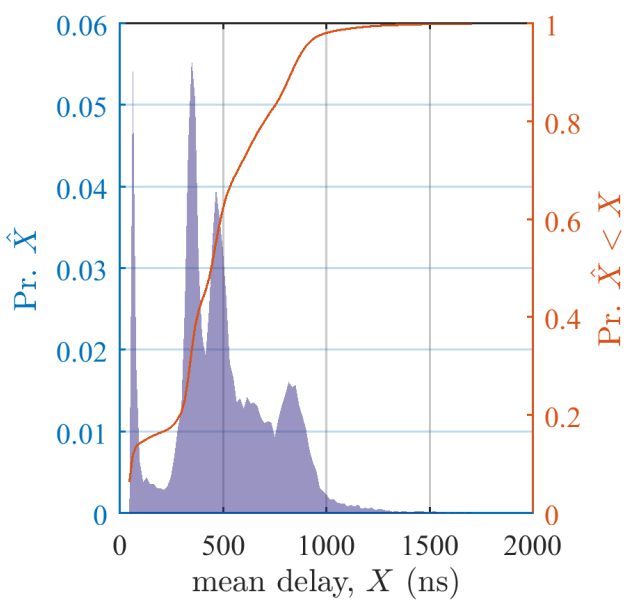

(c) Power Delay

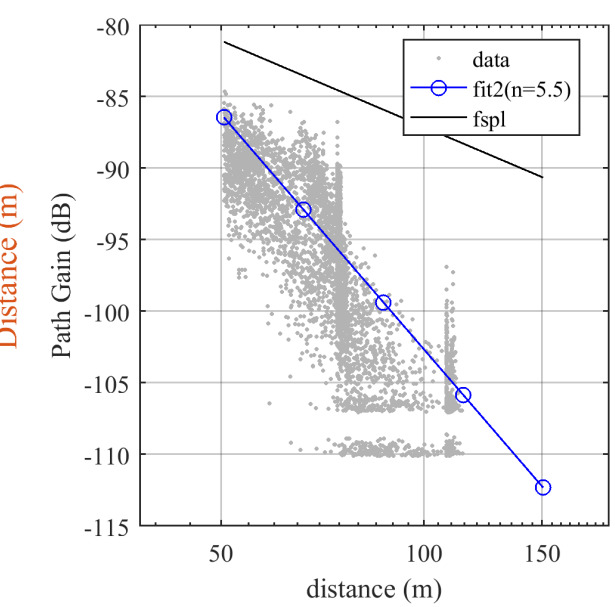

(b) Path Loss versus Distance

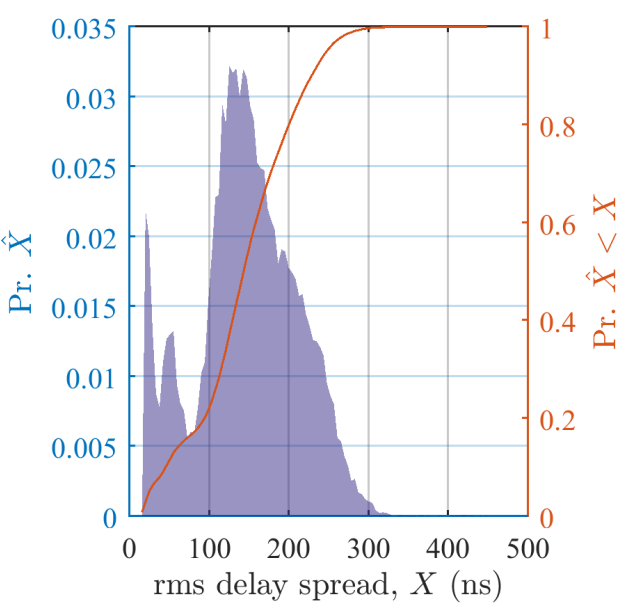

(d) Power Delay Spread

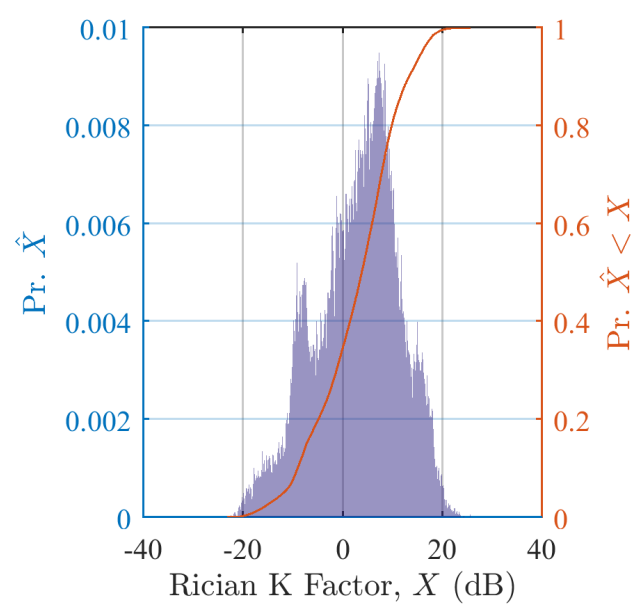

(e) Rician K-factor

Figure A.4.14: Channel characterizations for AAPlantD3 5GHz TX2b hpol internal run39 pp channel stats 


\section{A.4.15 AAPlantD3 5GHz TX2b hpol run38 pp.mat}

Table A.45: Measurement Parameters

\begin{tabular}{l|c}
\hline Parameter & Value \\
\hline \hline Mat File & 5.400 \\
Frequency $(\mathrm{GHz})$ & AAPlantD3_5GHz_TX2b_hpol_run38_pp.mat \\
Location & AA Plant Day 3 at Automotive Assembly Plant \\
RX Antenna & Omni-directional, Cross Pol \\
RX Antenna Gain & -3.500 \\
TX Antenna & Omni-directional, V Pol \\
TX Antenna Gain & 3.600 \\
TX Power, Watts & 1.250 \\
PN Oversample Factor & 4.000 \\
Sample rate, MHz & 80.000
\end{tabular}

Table A.46: Statistics of Channel Estimates. Outliers are removed using a significance test of 0.95 .

\begin{tabular}{l||c|c|c|c||c|c||l}
\hline Estimate & $\min (\cdot)$ & $\operatorname{median}(\cdot)$ & $\operatorname{mean}(\cdot)$ & $\max (\cdot)$ & $\operatorname{std}(\cdot)$ & $\operatorname{mad}(\cdot)$ & outlier info \\
\hline \hline$K(\mathrm{~dB})$, NLOS & -23.4 & 4.2 & 4.3 & 23.2 & 7.2 & 6.0 & N: 44275 \\
\hline$K(\mathrm{~dB})$, LOS & 0.0 & 11.7 & 11.2 & 23.2 & 3.1 & 2.5 & N: 17895 \\
\hline$\tau(\mathrm{ns})$ & 30.7 & 405.2 & 552.3 & 2870.8 & 477.4 & 411.3 & N: 43847 \\
\hline$S(\mathrm{~ns})$ & 0.0 & 124.9 & 138.6 & 475.9 & 70.0 & 60.8 & N: 40700 \\
\hline
\end{tabular}

Table A.47: Statistics of Channel Estimates. Outliers are not removed. N indicates the total number of samples in the population. Outliers are provided at 10 and 20 times $\sigma$.

\begin{tabular}{|c|c|c|c|c|c|c|c|}
\hline Estimate & $\min (\cdot)$ & $\operatorname{median}(\cdot)$ & mean $(\cdot)$ & $\max (\cdot)$ & $\operatorname{std}(\cdot)$ & $\operatorname{mad}(\cdot)$ & outlier info \\
\hline$K(\mathrm{~dB}), \mathrm{NLOS}$ & -23.4 & 4.2 & 4.3 & 23.2 & 7.2 & 6.0 & $\mathrm{~N}: 44275$ \\
\hline$K(\mathrm{~dB}), \operatorname{LOS}$ & 0.0 & 11.7 & 11.2 & 23.2 & 3.1 & 2.5 & $\mathrm{~N}: 17895$ \\
\hline$\tau(\mathrm{ns})$ & 30.7 & 418.0 & 652.5 & 80762.5 & 1745.8 & 518.9 & $\begin{array}{l}\mathrm{N}: 44395 \\
10 \sigma(18110.3): 54 \\
20 \sigma(35568.1): 27\end{array}$ \\
\hline$S(\mathrm{~ns})$ & 0.0 & 138.2 & 567.5 & 38848.0 & 1903.3 & 786.6 & $\begin{array}{l}\mathrm{N}: 44395 \\
10 \sigma(19600.8): 56 \\
20 \sigma(38634.1): 1\end{array}$ \\
\hline
\end{tabular}




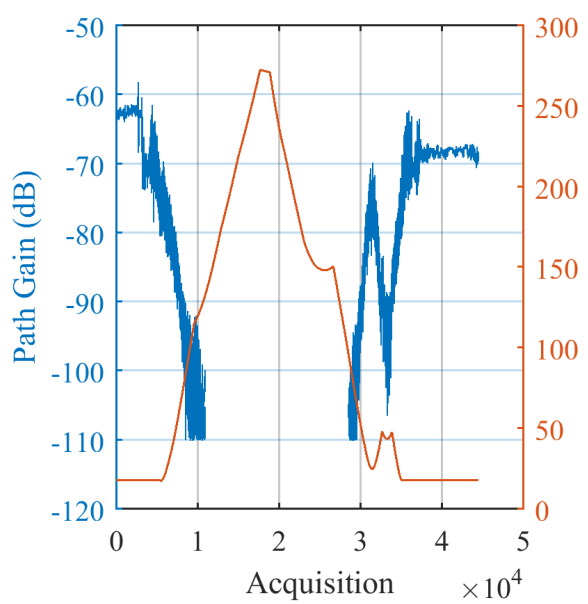

(a) Path Gain versus Acquisition Order

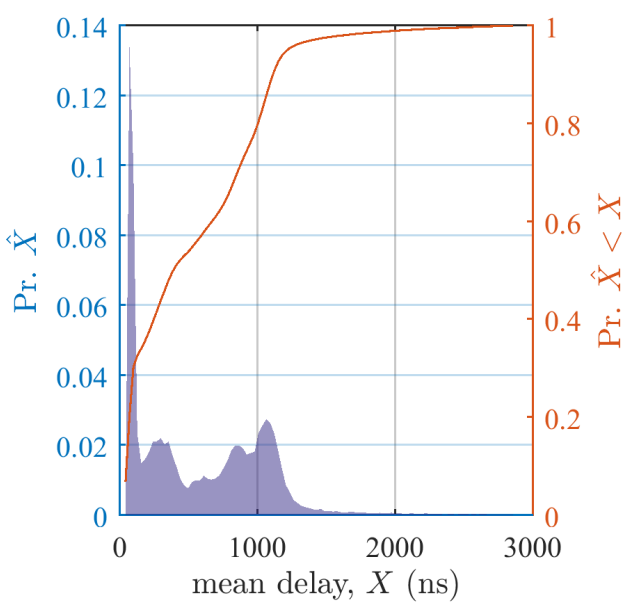

(c) Power Delay

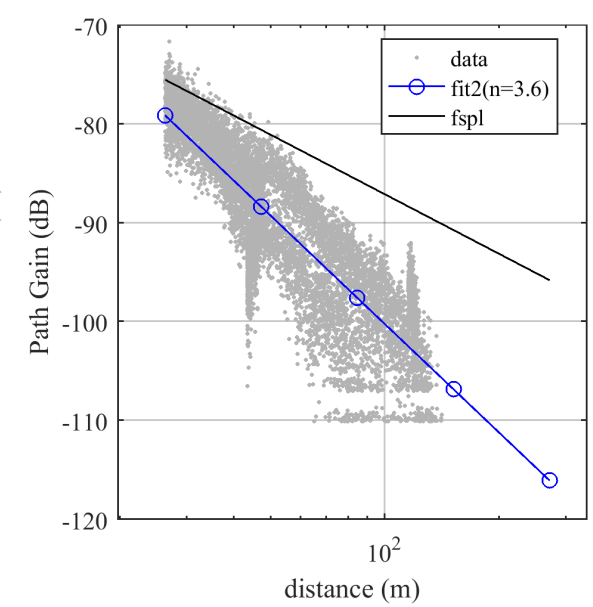

(b) Path Loss versus Distance

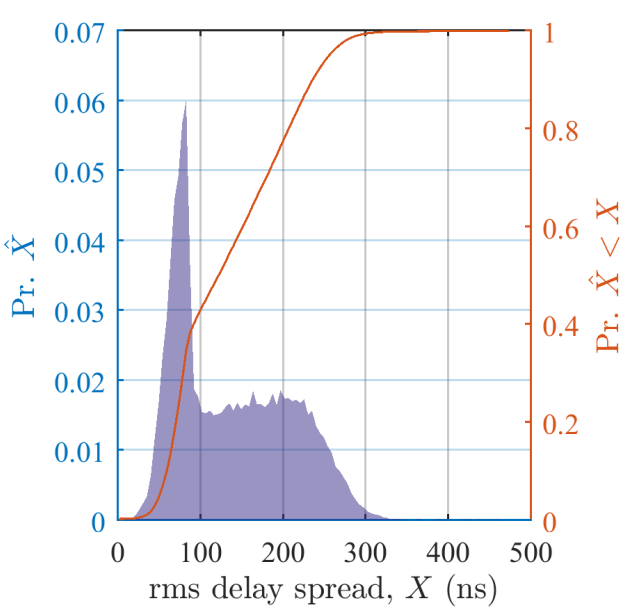

(d) Power Delay Spread

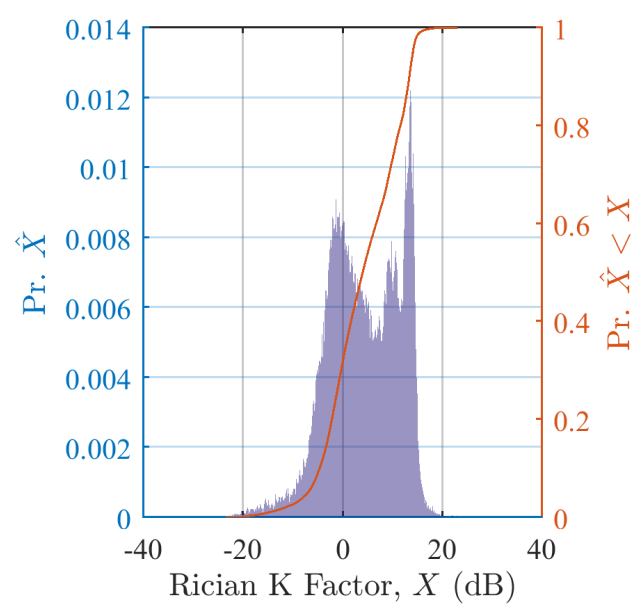

(e) Rician K-factor

Figure A.4.15: Channel characterizations for AAPlantD3 5GHz TX2b hpol run38 pp channel stats 


\section{A.4.16 AAPlantD3 5GHz TX2b vpol internal run40 pp.mat}

Table A.48: Measurement Parameters

\begin{tabular}{l|c}
\hline Parameter & Value \\
\hline \hline Mat File & AAPlantD3_5GHz_TX2b_vpol_interna \\
Frequency (GHz) & 5.400 \\
Location & AA Plant Day 3 at Automotive Ass \\
RX Antenna & Omni-directional, V Pol \\
RX Antenna Gain & -3.500 \\
TX Antenna & Omni-directional, V Pol \\
TX Antenna Gain & 3.600 \\
TX Power, Watts & 1.250 \\
PN Oversample Factor & 4.000 \\
Sample rate, MHz & 80.000
\end{tabular}

Table A.49: Statistics of Channel Estimates. Outliers are removed using a significance test of 0.95.

\begin{tabular}{l||c|c|c|c||c|c||l}
\hline Estimate & $\min (\cdot)$ & $\operatorname{median}(\cdot)$ & $\operatorname{mean}(\cdot)$ & $\max (\cdot)$ & $\operatorname{std}(\cdot)$ & $\operatorname{mad}(\cdot)$ & outlier info \\
\hline \hline$K(\mathrm{~dB})$, NLOS & -22.8 & 7.6 & 5.5 & 27.0 & 7.9 & 6.5 & N: 29700 \\
\hline$K(\mathrm{~dB})$, LOS & 3.6 & 11.8 & 11.9 & 23.3 & 2.5 & 1.9 & N: 13769 \\
\hline$\tau(\mathrm{ns})$ & 67.3 & 472.6 & 542.5 & 1421.8 & 244.2 & 205.6 & N: 29699 \\
\hline$S(\mathrm{~ns})$ & 14.3 & 148.6 & 154.6 & 426.1 & 63.8 & 50.4 & N: 29481 \\
\hline
\end{tabular}

Table A.50: Statistics of Channel Estimates. Outliers are not removed. N indicates the total number of samples in the population. Outliers are provided at 10 and 20 times $\sigma$.

\begin{tabular}{l||c|c|c|c||c|c||l}
\hline Estimate & $\min (\cdot)$ & $\operatorname{median}(\cdot)$ & $\operatorname{mean}(\cdot)$ & $\max (\cdot)$ & $\operatorname{std}(\cdot)$ & $\operatorname{mad}(\cdot)$ & outlier info \\
\hline \hline$K(\mathrm{~dB})$, NLOS & -22.8 & 7.6 & 5.5 & 27.0 & 7.9 & 6.5 & $\mathrm{~N}: 29700$ \\
\hline$K(\mathrm{~dB})$, LOS & 3.6 & 11.8 & 12.0 & 27.0 & 2.5 & 1.9 & $\mathrm{~N}: 13782$ \\
\hline$\tau(\mathrm{ns})$ & 67.3 & 472.6 & 542.5 & 1785.2 & 244.3 & 205.7 & $\mathrm{~N}: 29700$ \\
\hline$S(\mathrm{~ns})$ & 14.3 & 149.0 & 171.6 & 6030.5 & 226.8 & 70.4 & $\mathrm{~N}: 29700$ \\
& & & & & & & $10 \sigma(2439.6): 103$ \\
& & & & & & & $20 \sigma(4707.7): 3$ \\
\hline
\end{tabular}




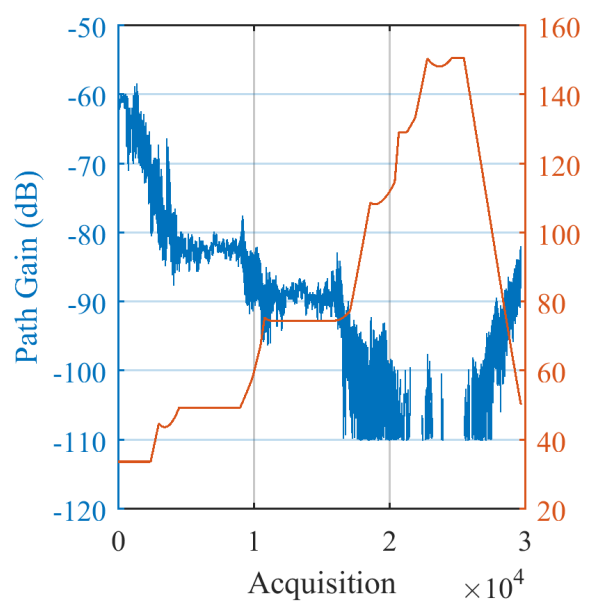

(a) Path Gain versus Acquisition Order

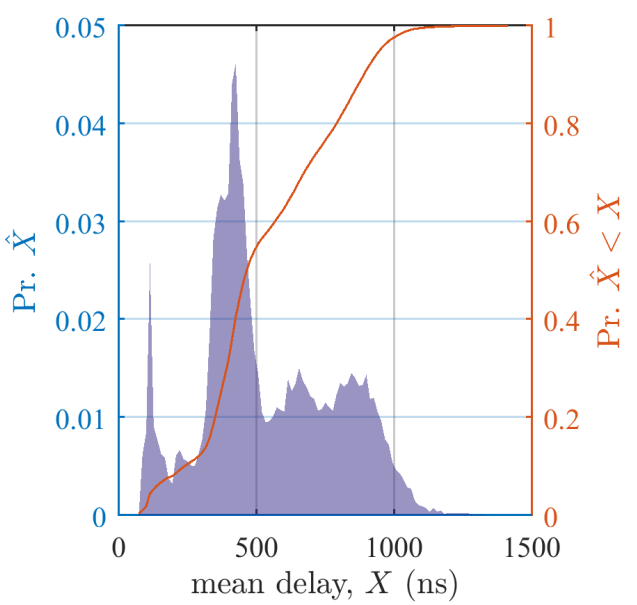

(c) Power Delay

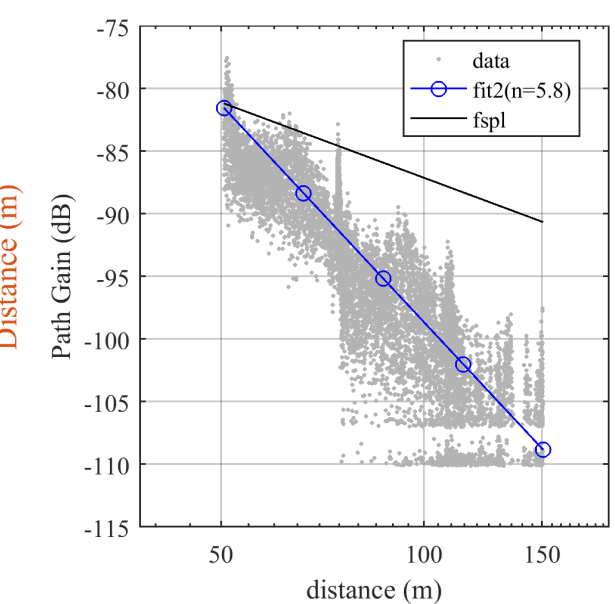

(b) Path Loss versus Distance

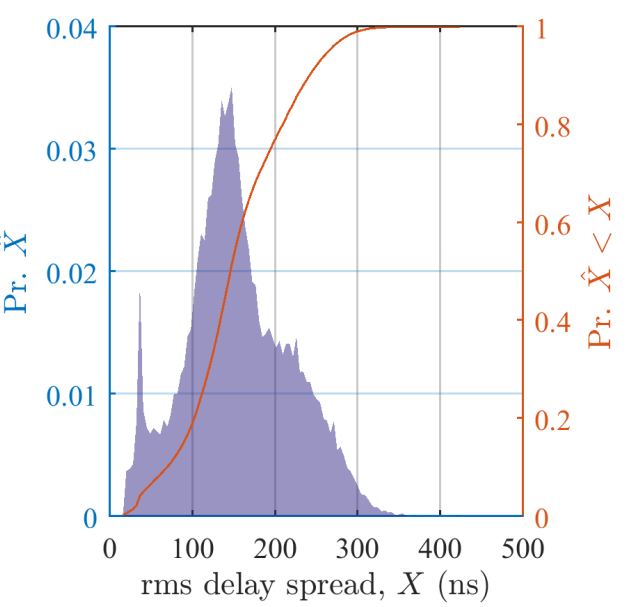

(d) Power Delay Spread

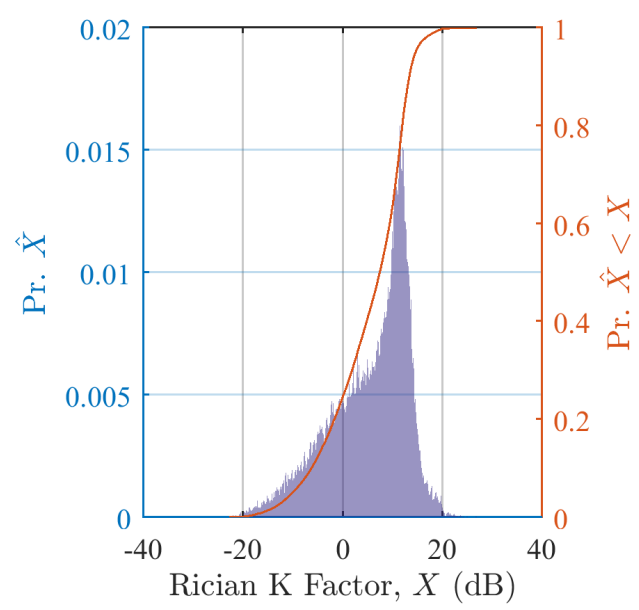

(e) Rician K-factor

Figure A.4.16: Channel characterizations for AAPlantD3 5GHz TX2b vpol internal run40 pp channel stats 


\section{A.4.17 AAPlantD3 5GHz TX2b vpol run37 pp.mat}

Table A.51: Measurement Parameters

\begin{tabular}{l|c}
\hline Parameter & Value \\
\hline \hline Mat File & AAPlantD3_5GHz_TX2b_vpol_run37_pp.mat \\
Frequency $(\mathrm{GHz})$ & 5.400 \\
Location & AA Plant Day 3 at Automotive Assembly Plant \\
RX Antenna & Omni-directional, V Pol \\
RX Antenna Gain & -3.500 \\
TX Antenna & Omni-directional, V Pol \\
TX Antenna Gain & 3.600 \\
TX Power, Watts & 1.250 \\
PN Oversample Factor & 4.000 \\
Sample rate, MHz & 80.000
\end{tabular}

Table A.52: Statistics of Channel Estimates. Outliers are removed using a significance test of 0.95.

\begin{tabular}{l||c|c|c|c||c|c||l}
\hline Estimate & $\min (\cdot)$ & $\operatorname{median}(\cdot)$ & $\operatorname{mean}(\cdot)$ & $\max (\cdot)$ & $\operatorname{std}(\cdot)$ & $\operatorname{mad}(\cdot)$ & outlier info \\
\hline \hline$K(\mathrm{~dB})$, NLOS & -23.3 & 5.1 & 5.2 & 27.0 & 8.6 & 7.1 & N: 38340 \\
\hline$K(\mathrm{~dB})$, LOS & 2.5 & 13.4 & 13.6 & 27.0 & 4.2 & 3.7 & N: 15523 \\
\hline$\tau(\mathrm{ns})$ & 20.7 & 690.3 & 630.7 & 2569.1 & 401.2 & 355.3 & N: 38328 \\
\hline$S(\mathrm{~ns})$ & 13.8 & 160.9 & 153.2 & 537.9 & 80.7 & 69.1 & N: 37066 \\
\hline
\end{tabular}

Table A.53: Statistics of Channel Estimates. Outliers are not removed. N indicates the total number of samples in the population. Outliers are provided at 10 and 20 times $\sigma$.

\begin{tabular}{l||c|c|c|c||c|c||l}
\hline Estimate & $\min (\cdot)$ & $\operatorname{median}(\cdot)$ & $\operatorname{mean}(\cdot)$ & $\max (\cdot)$ & $\operatorname{std}(\cdot)$ & $\operatorname{mad}(\cdot)$ & outlier info \\
\hline \hline$K(\mathrm{~dB})$, NLOS & -23.3 & 5.1 & 5.2 & 27.0 & 8.6 & 7.1 & N: 38340 \\
\hline$K(\mathrm{~dB})$, LOS & 2.5 & 13.4 & 13.6 & 27.0 & 4.2 & 3.7 & N: 15523 \\
\hline$\tau(\mathrm{ns})$ & 20.7 & 690.6 & 631.4 & 3449.5 & 403.2 & 355.9 & $\mathrm{~N}: 38340$ \\
\hline$S(\mathrm{~ns})$ & 13.8 & 165.5 & 246.0 & 11532.4 & 594.2 & 186.4 & $\mathrm{~N}: 38340$ \\
& & & & & & & $10 \sigma(6187.8): 59$ \\
\hline
\end{tabular}




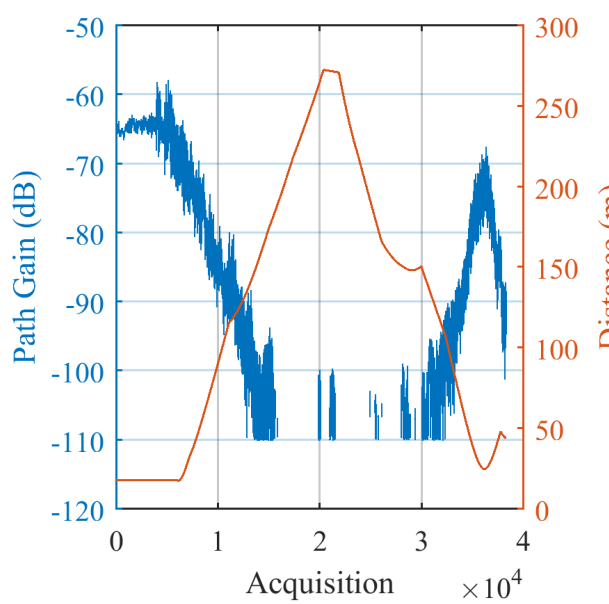

(a) Path Gain versus Acquisition Order

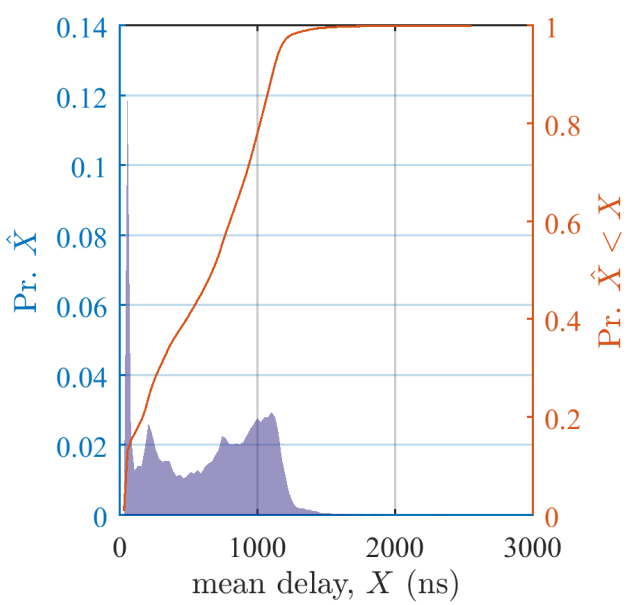

(c) Power Delay

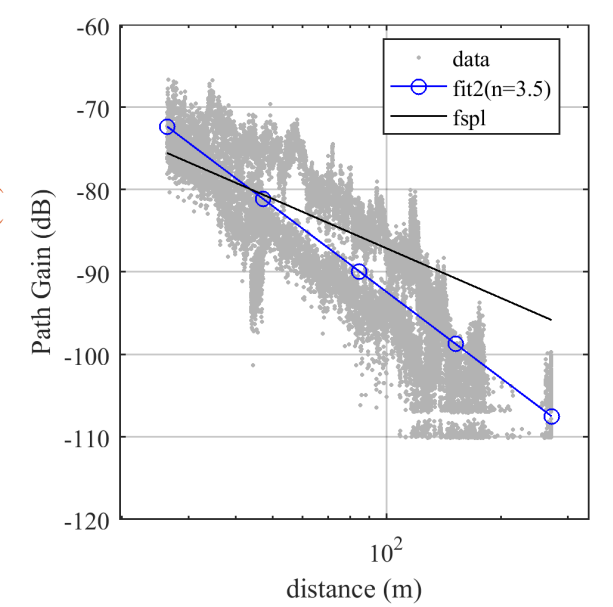

(b) Path Loss versus Distance

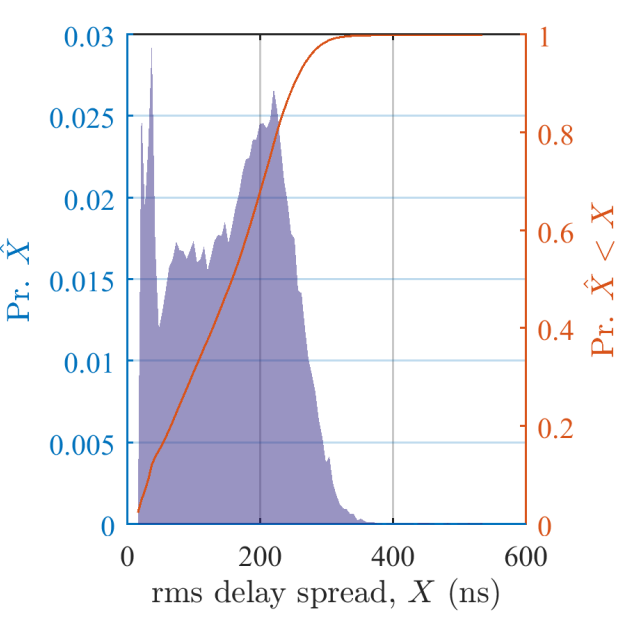

(d) Power Delay Spread

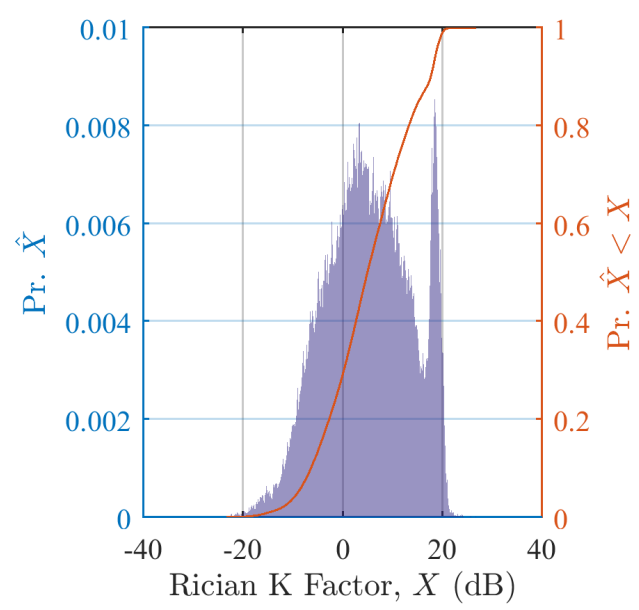

(e) Rician K-factor

Figure A.4.17: Channel characterizations for AAPlantD3 5GHz TX2b vpol run37 pp channel stats 


\section{A.4.18 GBurgD2 2GHz TX1 crossPol run3 pp.mat}

Table A.54: Measurement Parameters

\begin{tabular}{l|c}
\hline Parameter & Value \\
\hline \hline Mat File & GBurgD2_2GHz_TX1_crossPol_run3_pp.mat \\
Frequency (GHz) & 2.245 \\
Location & Machine Shop Day2 at Gaithersburg \\
RX Antenna & Omni-directional, Cross Pol \\
RX Antenna Gain & -4.200 \\
TX Antenna & Omni-directional, V Pol \\
TX Antenna Gain & 2.900 \\
TX Power, Watts & 1.500 \\
PN Oversample Factor & 4.000 \\
Sample rate, MHz & 80.000
\end{tabular}

Table A.55: Statistics of Channel Estimates. Outliers are removed using a significance test of 0.95.

\begin{tabular}{l||c|c|c|c||c|c||l}
\hline Estimate & $\min (\cdot)$ & $\operatorname{median}(\cdot)$ & $\operatorname{mean}(\cdot)$ & $\max (\cdot)$ & $\operatorname{std}(\cdot)$ & $\operatorname{mad}(\cdot)$ & outlier info \\
\hline \hline$K(\mathrm{~dB})$, NLOS & -25.1 & 6.2 & 3.9 & 27.2 & 9.3 & 7.0 & N: 11192 \\
\hline$K(\mathrm{~dB})$, LOS & 3.8 & 10.3 & 10.8 & 24.7 & 3.2 & 2.5 & N: 5189 \\
\hline$\tau(\mathrm{ns})$ & 11.1 & 188.2 & 182.0 & 383.6 & 60.1 & 48.8 & N: 11192 \\
\hline$S(\mathrm{~ns})$ & 9.7 & 77.3 & 76.6 & 156.4 & 21.9 & 17.5 & N: 11192 \\
\hline
\end{tabular}

Table A.56: Statistics of Channel Estimates. Outliers are not removed. N indicates the total number of samples in the population. Outliers are provided at 10 and 20 times $\sigma$.

\begin{tabular}{l||c|c|c|c||c|c||l}
\hline Estimate & $\min (\cdot)$ & $\operatorname{median}(\cdot)$ & $\operatorname{mean}(\cdot)$ & $\max (\cdot)$ & $\operatorname{std}(\cdot)$ & $\operatorname{mad}(\cdot)$ & outlier info \\
\hline \hline$K(\mathrm{~dB})$, NLOS & -25.1 & 6.2 & 3.9 & 27.2 & 9.3 & 7.0 & N: 11192 \\
\hline$K(\mathrm{~dB})$, LOS & 3.8 & 10.3 & 10.8 & 27.2 & 3.2 & 2.5 & N: 5194 \\
\hline$\tau(\mathrm{ns})$ & 11.1 & 188.2 & 182.0 & 383.6 & 60.1 & 48.8 & N: 11192 \\
\hline$S(\mathrm{~ns})$ & 9.7 & 77.3 & 76.6 & 156.4 & 21.9 & 17.5 & N: 11192 \\
\hline
\end{tabular}




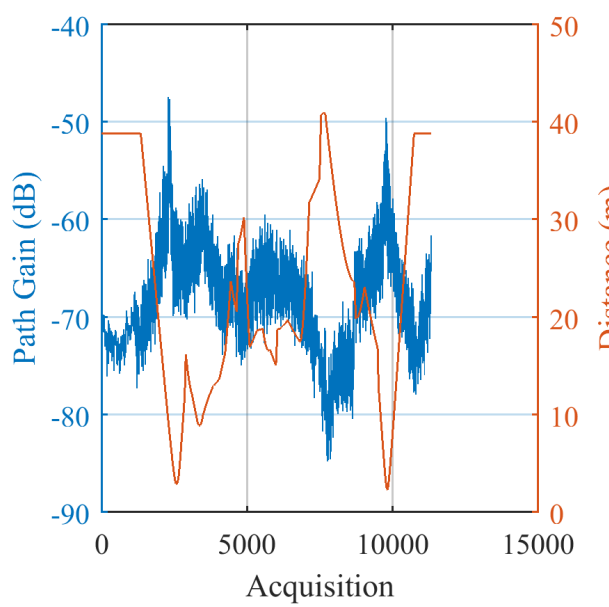

(a) Path Gain versus Acquisition Order

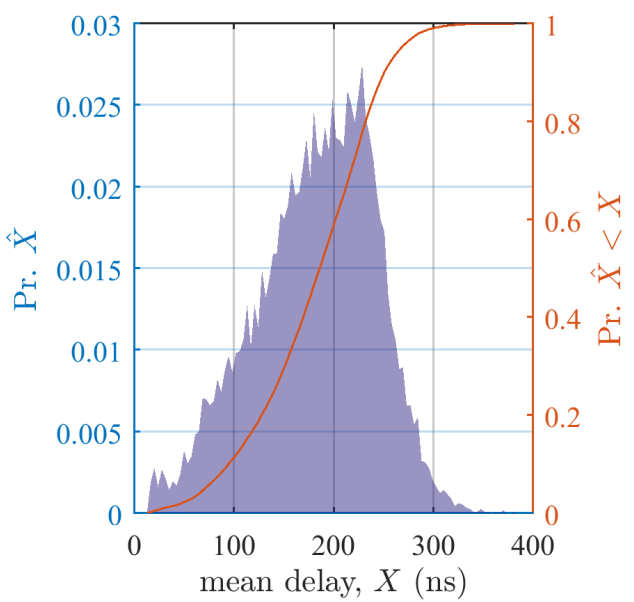

(c) Power Delay

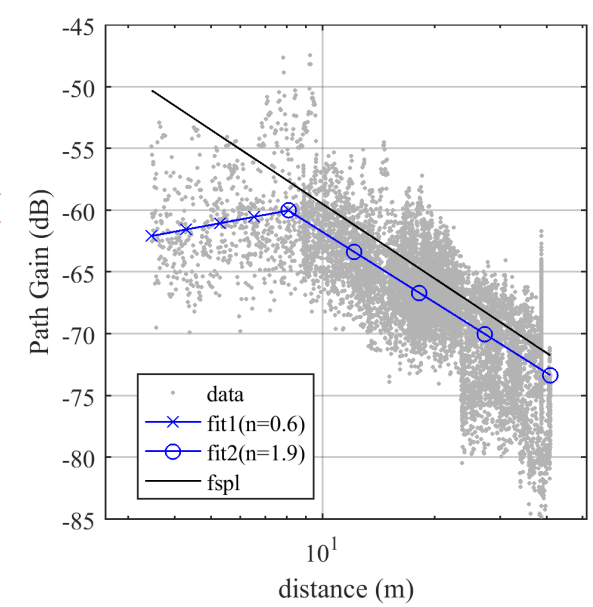

(b) Path Loss versus Distance

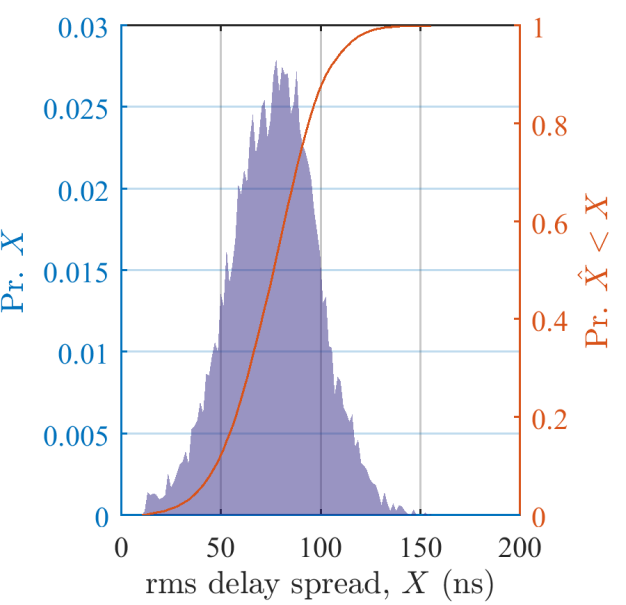

(d) Power Delay Spread

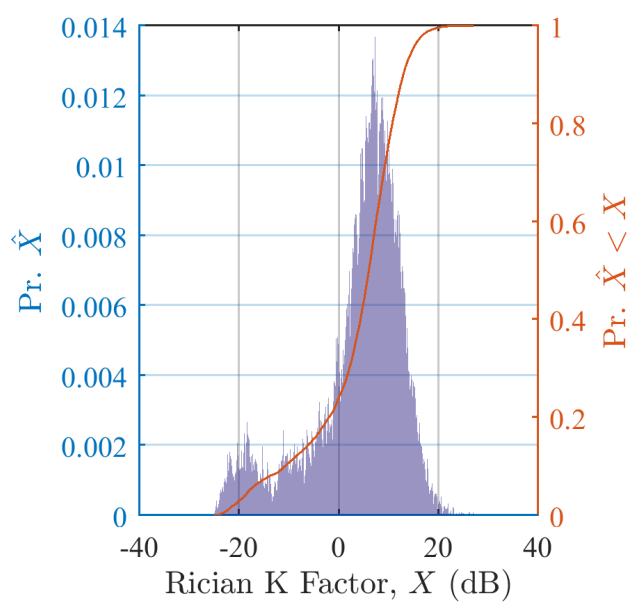

(e) Rician K-factor

Figure A.4.18: Channel characterizations for GBurgD2 2GHz TX1 crossPol run3 pp channel stats 


\section{A.4.19 GBurgD2 2GHz TX1 longPol run2 pp.mat}

Table A.57: Measurement Parameters

\begin{tabular}{l|c}
\hline Parameter & Value \\
\hline \hline Mat File & GBurgD2_2GHz_TX1_longPol_run2_pp.mat \\
Frequency (GHz) & 2.245 \\
Location & Machine Shop Day2 at Gaithersburg \\
RX Antenna & Omni-directional, Long Pol \\
RX Antenna Gain & -4.200 \\
TX Antenna & Omni-directional, V Pol \\
TX Antenna Gain & 2.900 \\
TX Power, Watts & 1.500 \\
PN Oversample Factor & 4.000 \\
Sample rate, MHz & 80.000
\end{tabular}

Table A.58: Statistics of Channel Estimates. Outliers are removed using a significance test of 0.95.

\begin{tabular}{l||c|c|c|c||c|c||l}
\hline Estimate & $\min (\cdot)$ & $\operatorname{median}(\cdot)$ & $\operatorname{mean}(\cdot)$ & $\max (\cdot)$ & $\operatorname{std}(\cdot)$ & $\operatorname{mad}(\cdot)$ & outlier info \\
\hline \hline$K(\mathrm{~dB})$, NLOS & -24.9 & 6.4 & 4.9 & 24.6 & 7.9 & 6.0 & N: 9031 \\
\hline$K(\mathrm{~dB})$, LOS & 2.0 & 10.5 & 10.9 & 24.6 & 3.2 & 2.6 & N: 4330 \\
\hline$\tau(\mathrm{ns})$ & 8.9 & 181.4 & 175.2 & 369.4 & 65.5 & 53.1 & N: 9031 \\
\hline$S(\mathrm{~ns})$ & 10.1 & 77.5 & 76.7 & 169.3 & 23.1 & 18.6 & N: 9031 \\
\hline
\end{tabular}

Table A.59: Statistics of Channel Estimates. Outliers are not removed. N indicates the total number of samples in the population. Outliers are provided at 10 and 20 times $\sigma$.

\begin{tabular}{l||c|c|c|c||c|c||l}
\hline Estimate & $\min (\cdot)$ & $\operatorname{median}(\cdot)$ & $\operatorname{mean}(\cdot)$ & $\max (\cdot)$ & $\operatorname{std}(\cdot)$ & $\operatorname{mad}(\cdot)$ & outlier info \\
\hline \hline$K(\mathrm{~dB})$, NLOS & -24.9 & 6.4 & 4.9 & 24.6 & 7.9 & 6.0 & N: 9031 \\
\hline$K(\mathrm{~dB})$, LOS & 2.0 & 10.5 & 10.9 & 24.6 & 3.2 & 2.6 & N: 4330 \\
\hline$\tau(\mathrm{ns})$ & 8.9 & 181.4 & 175.2 & 369.4 & 65.5 & 53.1 & N: 9031 \\
\hline$S(\mathrm{~ns})$ & 10.1 & 77.5 & 76.7 & 169.3 & 23.1 & 18.6 & N: 9031 \\
\hline
\end{tabular}




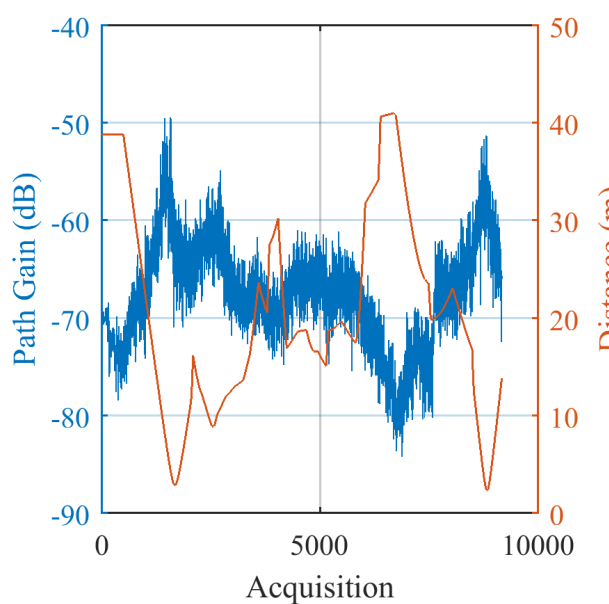

(a) Path Gain versus Acquisition Order

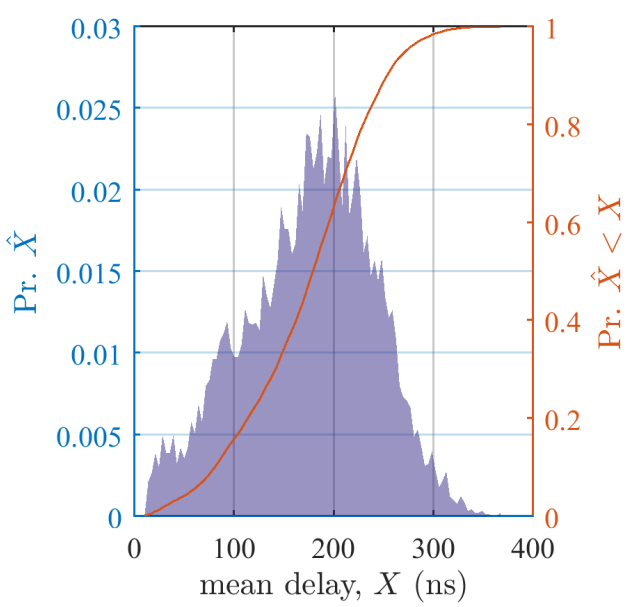

(c) Power Delay

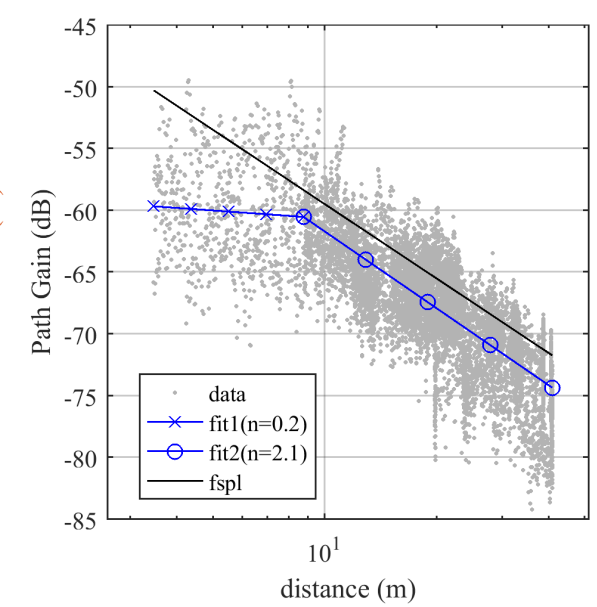

(b) Path Loss versus Distance

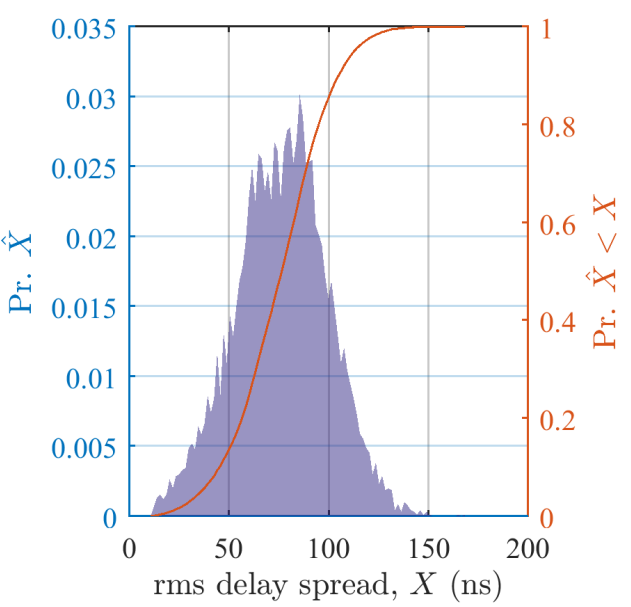

(d) Power Delay Spread

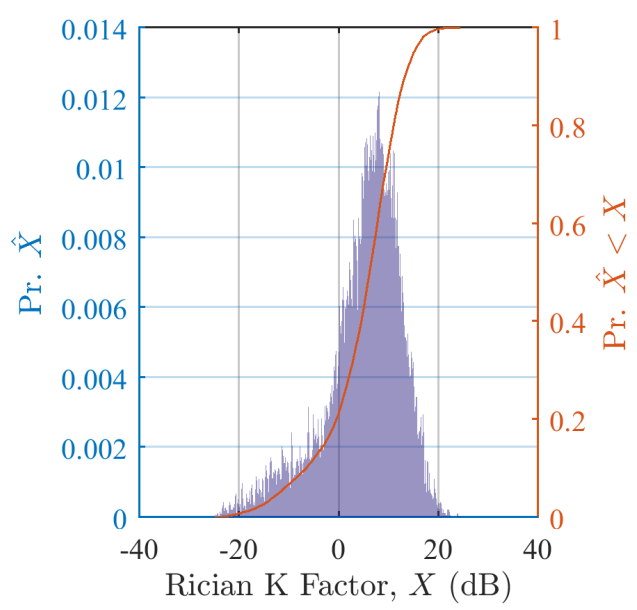

(e) Rician K-factor

Figure A.4.19: Channel characterizations for GBurgD2 2GHz TX1 longPol run2 pp channel stats 


\section{A.4.20 GBurgD2 2GHz TX1 vpol run1again pp.mat}

Table A.60: Measurement Parameters

\begin{tabular}{l|c}
\hline Parameter & Value \\
\hline \hline Mat File & GBurgD2_2GHz_TX1_vpol_run1again_pp.mat \\
Frequency $(\mathrm{GHz})$ & 2.245 \\
Location & Machine Shop Day2 at Gaithersburg \\
RX Antenna & Omni-directional, V Pol \\
RX Antenna Gain & -4.200 \\
TX Antenna & Omni-directional, V Pol \\
TX Antenna Gain & 2.900 \\
TX Power, Watts & 1.500 \\
PN Oversample Factor & 4.000 \\
Sample rate, MHz & 80.000
\end{tabular}

Table A.61: Statistics of Channel Estimates. Outliers are removed using a significance test of 0.95.

\begin{tabular}{l||c|c|c|c||c|c||l}
\hline Estimate & $\min (\cdot)$ & $\operatorname{median}(\cdot)$ & $\operatorname{mean}(\cdot)$ & $\max (\cdot)$ & $\operatorname{std}(\cdot)$ & $\operatorname{mad}(\cdot)$ & outlier info \\
\hline \hline$K(\mathrm{~dB})$, NLOS & -24.6 & 9.6 & 8.7 & 29.8 & 7.5 & 5.6 & N: 9307 \\
\hline$K(\mathrm{~dB})$, LOS & 1.8 & 11.9 & 12.5 & 29.8 & 4.2 & 3.4 & N: 6411 \\
\hline$\tau(\mathrm{ns})$ & 7.8 & 142.9 & 143.8 & 354.4 & 70.2 & 58.3 & N: 9309 \\
\hline$S(\mathrm{~ns})$ & 9.9 & 63.1 & 63.6 & 150.3 & 27.1 & 22.5 & N: 9309 \\
\hline
\end{tabular}

Table A.62: Statistics of Channel Estimates. Outliers are not removed. N indicates the total number of samples in the population. Outliers are provided at 10 and 20 times $\sigma$.

\begin{tabular}{l||c|c|c|c||c|c||l}
\hline Estimate & $\min (\cdot)$ & $\operatorname{median}(\cdot)$ & $\operatorname{mean}(\cdot)$ & $\max (\cdot)$ & $\operatorname{std}(\cdot)$ & $\operatorname{mad}(\cdot)$ & outlier info \\
\hline \hline$K(\mathrm{~dB})$, NLOS & -24.6 & 9.6 & 8.7 & 29.8 & 7.5 & 5.6 & N: 9307 \\
\hline$K(\mathrm{~dB})$, LOS & 1.8 & 11.9 & 12.5 & 29.8 & 4.2 & 3.4 & N: 6411 \\
\hline$\tau(\mathrm{ns})$ & 7.8 & 142.9 & 143.8 & 354.4 & 70.2 & 58.3 & N: 9309 \\
\hline$S(\mathrm{~ns})$ & 9.9 & 63.1 & 63.6 & 150.3 & 27.1 & 22.5 & N: 9309 \\
\hline
\end{tabular}




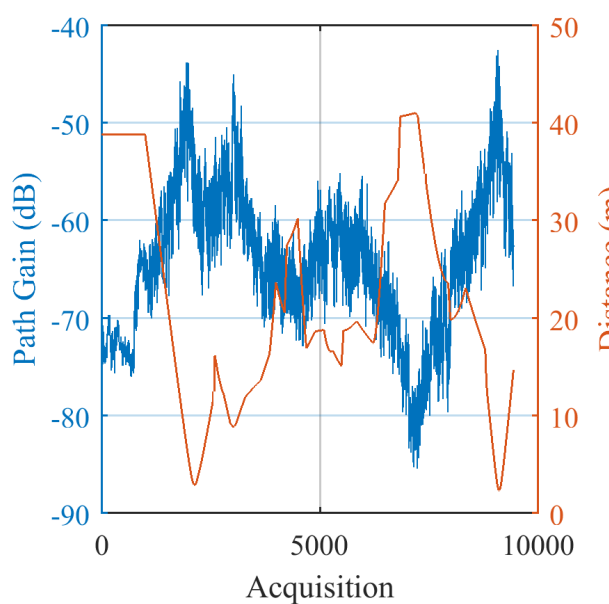

(a) Path Gain versus Acquisition Order

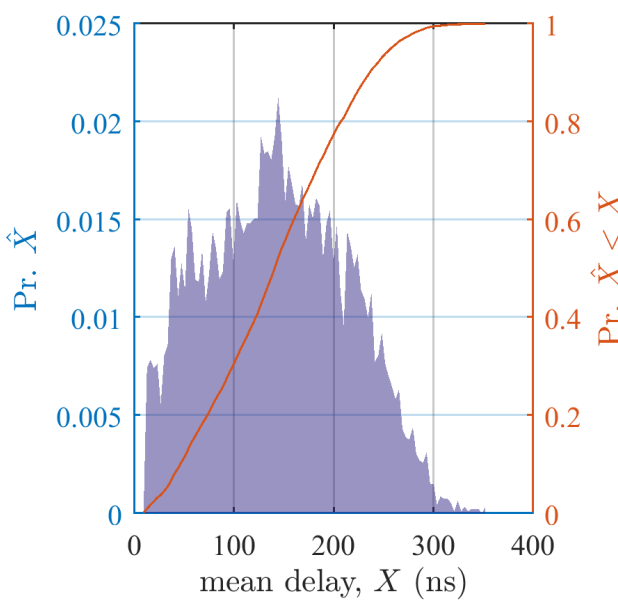

(c) Power Delay

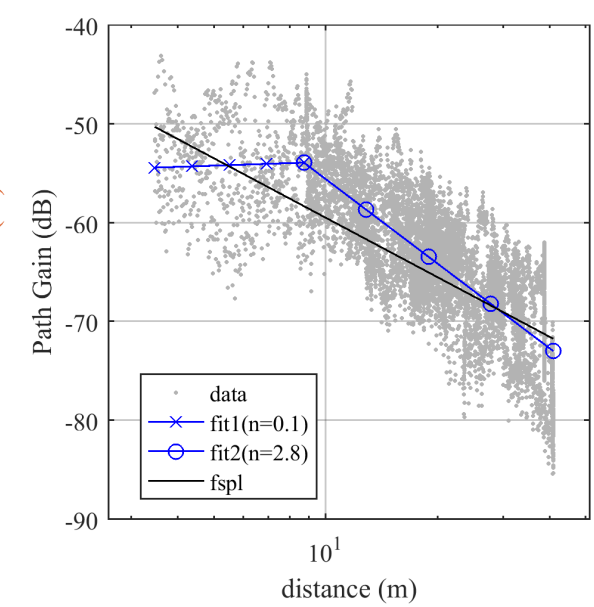

(b) Path Loss versus Distance

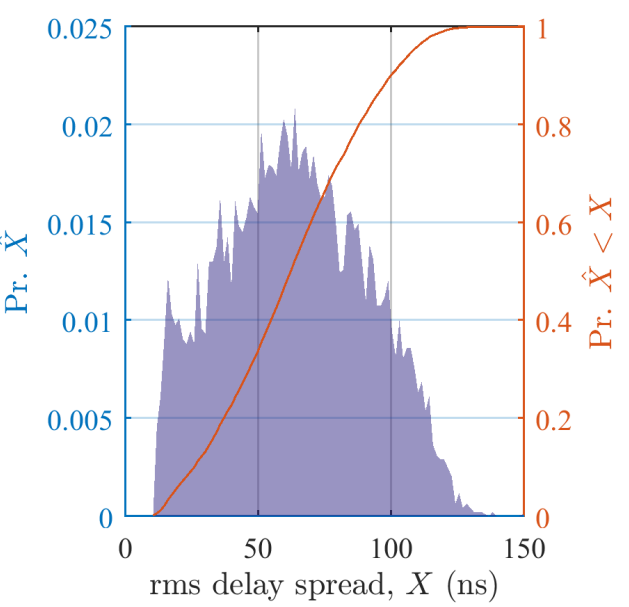

(d) Power Delay Spread

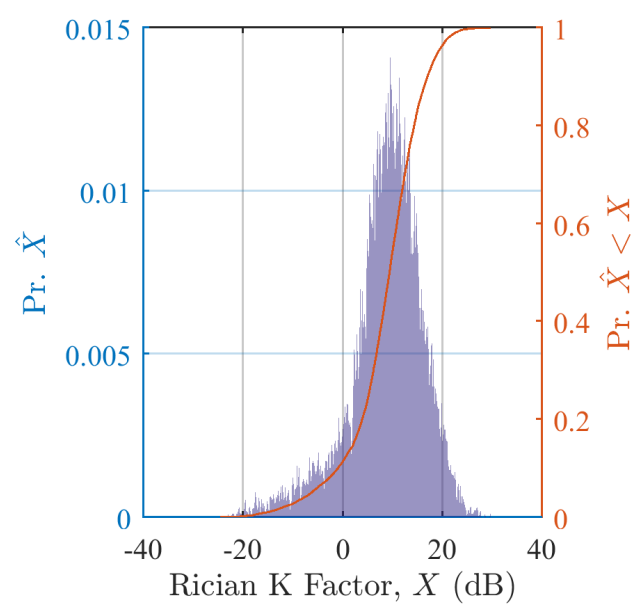

(e) Rician K-factor

Figure A.4.20: Channel characterizations for GBurgD2 2GHz TX1 vpol run1again pp channel stats 


\section{A.4.21 GBurgD2 5GHz TX1 crossPol run3 pp.mat}

Table A.63: Measurement Parameters

\begin{tabular}{l|c}
\hline Parameter & Value \\
\hline \hline Mat File & GBurgD2_5GHz_TX1_crossPol_run3_pp.mat \\
Frequency (GHz) & 5.400 \\
Location & Machine Shop Day2 at Gaithersburg \\
RX Antenna & Omni-directional, Cross Pol \\
RX Antenna Gain & -3.500 \\
TX Antenna & Omni-directional, V Pol \\
TX Antenna Gain & 3.600 \\
TX Power, Watts & 1.250 \\
PN Oversample Factor & 4.000 \\
Sample rate, MHz & 80.000
\end{tabular}

Table A.64: Statistics of Channel Estimates. Outliers are removed using a significance test of 0.95.

\begin{tabular}{l||c|c|c|c||c|c||l}
\hline Estimate & $\min (\cdot)$ & $\operatorname{median}(\cdot)$ & $\operatorname{mean}(\cdot)$ & $\max (\cdot)$ & $\operatorname{std}(\cdot)$ & $\operatorname{mad}(\cdot)$ & outlier info \\
\hline \hline$K(\mathrm{~dB})$, NLOS & -25.0 & 6.4 & 5.0 & 26.0 & 7.8 & 6.0 & N: 10237 \\
\hline$K(\mathrm{~dB})$, LOS & 3.6 & 10.4 & 10.8 & 24.6 & 3.2 & 2.6 & N: 4920 \\
\hline$\tau(\mathrm{ns})$ & 12.6 & 185.7 & 183.7 & 378.0 & 61.2 & 48.5 & N: 10237 \\
\hline$S(\mathrm{~ns})$ & 10.0 & 74.7 & 75.2 & 155.3 & 21.6 & 17.4 & N: 10237 \\
\hline
\end{tabular}

Table A.65: Statistics of Channel Estimates. Outliers are not removed. N indicates the total number of samples in the population. Outliers are provided at 10 and 20 times $\sigma$.

\begin{tabular}{l||c|c|c|c||c|c||l}
\hline Estimate & $\min (\cdot)$ & $\operatorname{median}(\cdot)$ & $\operatorname{mean}(\cdot)$ & $\max (\cdot)$ & $\operatorname{std}(\cdot)$ & $\operatorname{mad}(\cdot)$ & outlier info \\
\hline \hline$K(\mathrm{~dB})$, NLOS & -25.0 & 6.4 & 5.0 & 26.0 & 7.8 & 6.0 & N: 10237 \\
\hline$K(\mathrm{~dB})$, LOS & 3.6 & 10.4 & 10.8 & 26.0 & 3.2 & 2.6 & N: 4923 \\
\hline$\tau(\mathrm{ns})$ & 12.6 & 185.7 & 183.7 & 378.0 & 61.2 & 48.5 & N: 10237 \\
\hline$S(\mathrm{~ns})$ & 10.0 & 74.7 & 75.2 & 155.3 & 21.6 & 17.4 & N: 10237 \\
\hline
\end{tabular}




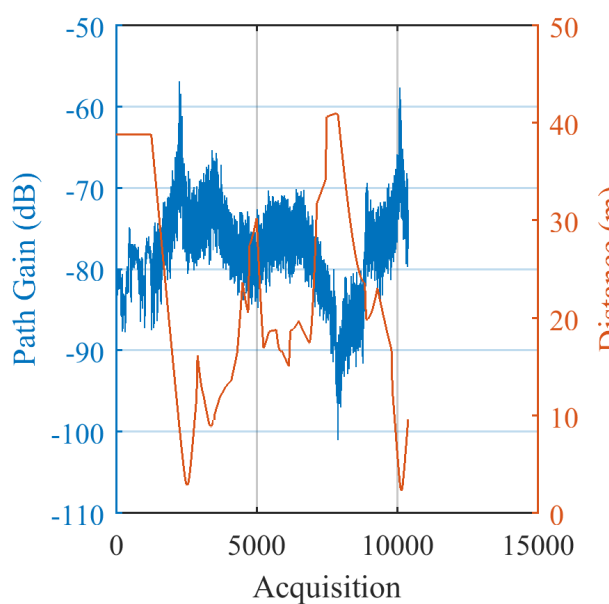

(a) Path Gain versus Acquisition Order

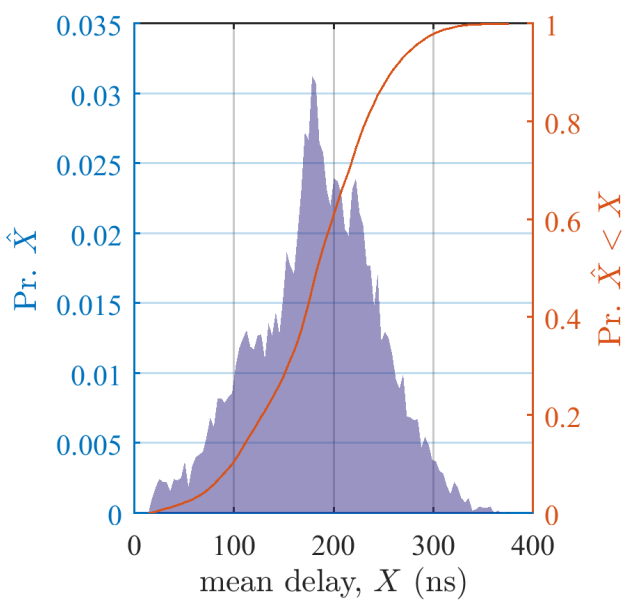

(c) Power Delay

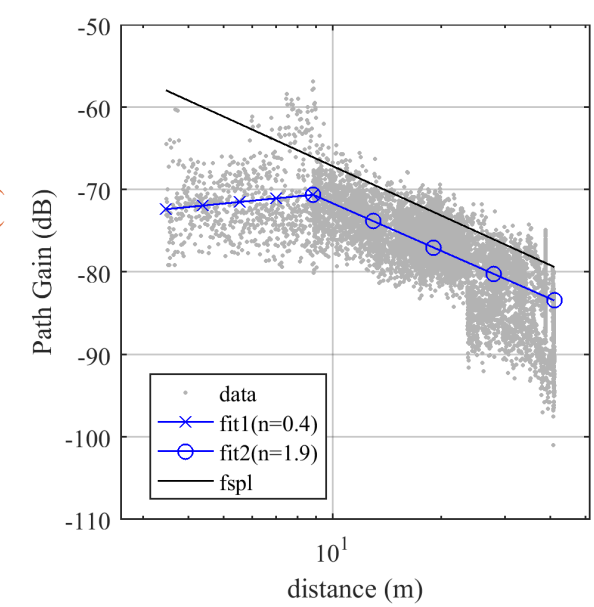

(b) Path Loss versus Distance

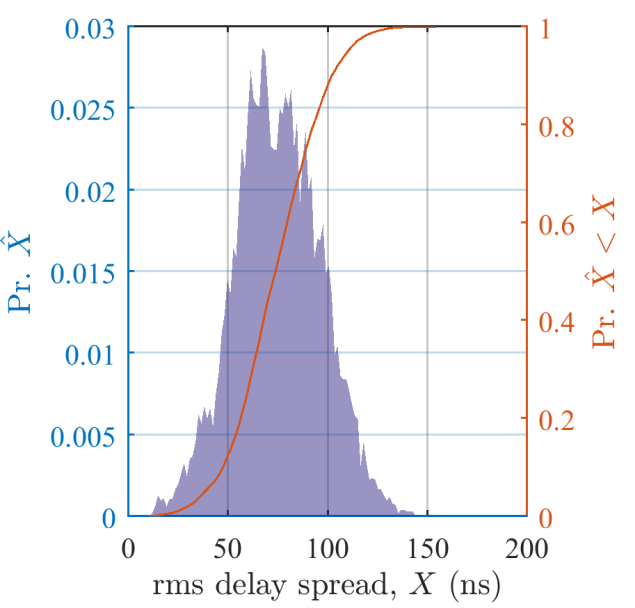

(d) Power Delay Spread

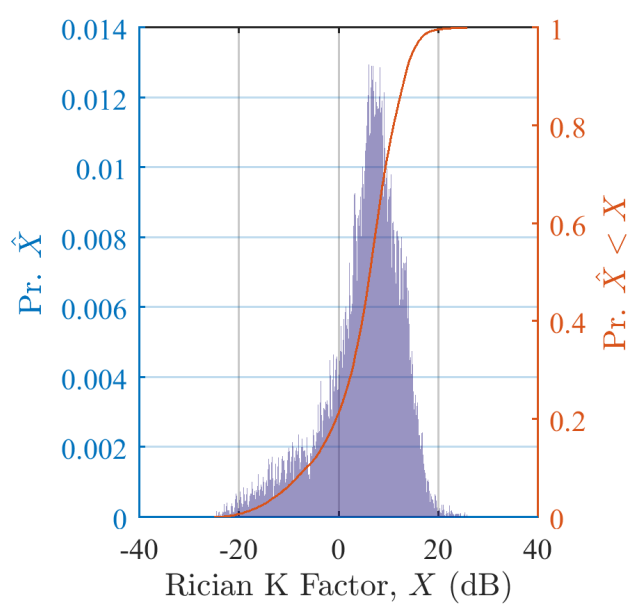

(e) Rician K-factor

Figure A.4.21: Channel characterizations for GBurgD2 5GHz TX1 crossPol run3 pp channel stats 


\section{A.4.22 GBurgD2 5GHz TX1 longPol run4 pp.mat}

Table A.66: Measurement Parameters

\begin{tabular}{l|c}
\hline Parameter & Value \\
\hline \hline Mat File & GBurgD2_5GHz_TX1_longPol_run4_pp.mat \\
Frequency (GHz) & 5.400 \\
Location & Machine Shop Day2 at Gaithersburg \\
RX Antenna & Omni-directional, Long Pol \\
RX Antenna Gain & -3.500 \\
TX Antenna & Omni-directional, V Pol \\
TX Antenna Gain & 3.600 \\
TX Power, Watts & 1.250 \\
PN Oversample Factor & 4.000 \\
Sample rate, MHz & 80.000
\end{tabular}

Table A.67: Statistics of Channel Estimates. Outliers are removed using a significance test of 0.95.

\begin{tabular}{l||c|c|c|c||c|c||l}
\hline Estimate & $\min (\cdot)$ & $\operatorname{median}(\cdot)$ & $\operatorname{mean}(\cdot)$ & $\max (\cdot)$ & $\operatorname{std}(\cdot)$ & $\operatorname{mad}(\cdot)$ & outlier info \\
\hline \hline$K(\mathrm{~dB})$, NLOS & -24.0 & 5.9 & 4.3 & 25.2 & 8.0 & 6.1 & N: 10336 \\
\hline$K(\mathrm{~dB})$, LOS & 3.3 & 10.1 & 10.6 & 24.7 & 3.2 & 2.6 & N: 4667 \\
\hline$\tau(\mathrm{ns})$ & 18.6 & 192.1 & 185.2 & 388.9 & 60.4 & 48.5 & N: 10336 \\
\hline$S(\mathrm{~ns})$ & 13.1 & 73.1 & 73.6 & 159.3 & 21.7 & 17.5 & N: 10336 \\
\hline
\end{tabular}

Table A.68: Statistics of Channel Estimates. Outliers are not removed. N indicates the total number of samples in the population. Outliers are provided at 10 and 20 times $\sigma$.

\begin{tabular}{l||c|c|c|c||c|c||l}
\hline Estimate & $\min (\cdot)$ & $\operatorname{median}(\cdot)$ & $\operatorname{mean}(\cdot)$ & $\max (\cdot)$ & $\operatorname{std}(\cdot)$ & $\operatorname{mad}(\cdot)$ & outlier info \\
\hline \hline$K(\mathrm{~dB})$, NLOS & -24.0 & 5.9 & 4.3 & 25.2 & 8.0 & 6.1 & N: 10336 \\
\hline$K(\mathrm{~dB})$, LOS & 3.3 & 10.1 & 10.6 & 25.2 & 3.2 & 2.6 & N: 4669 \\
\hline$\tau(\mathrm{ns})$ & 18.6 & 192.1 & 185.2 & 388.9 & 60.4 & 48.5 & N: 10336 \\
\hline$S(\mathrm{~ns})$ & 13.1 & 73.1 & 73.6 & 159.3 & 21.7 & 17.5 & N: 10336 \\
\hline
\end{tabular}




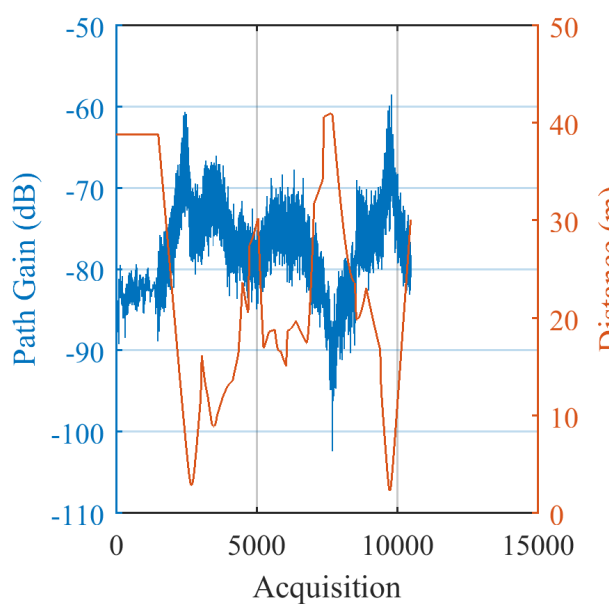

(a) Path Gain versus Acquisition Order

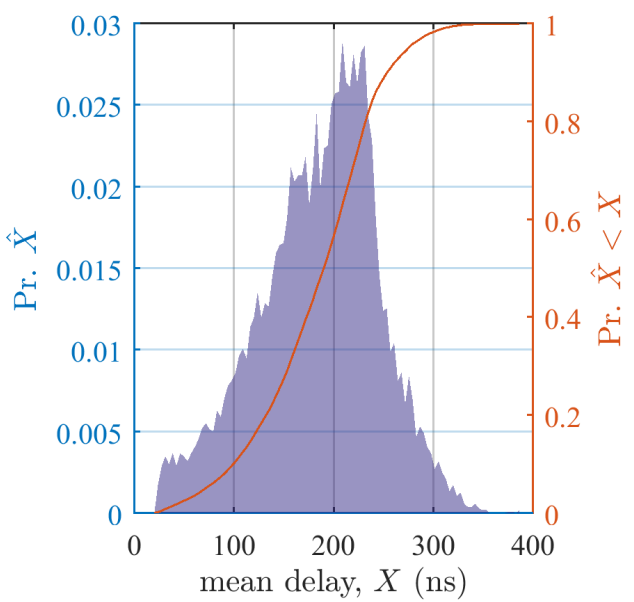

(c) Power Delay

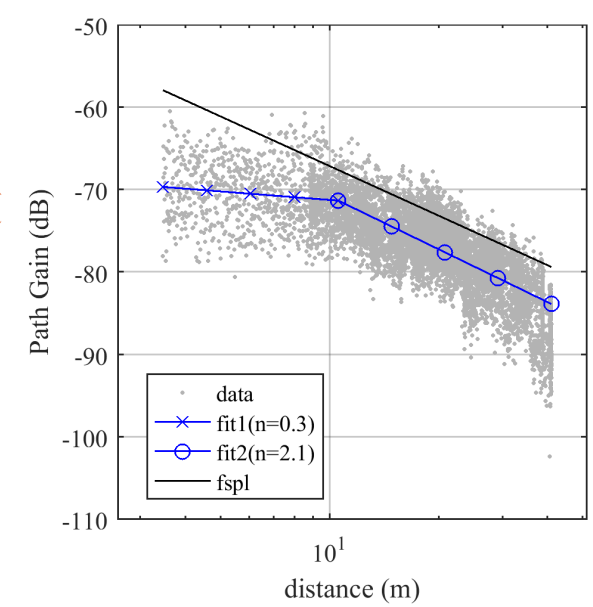

(b) Path Loss versus Distance

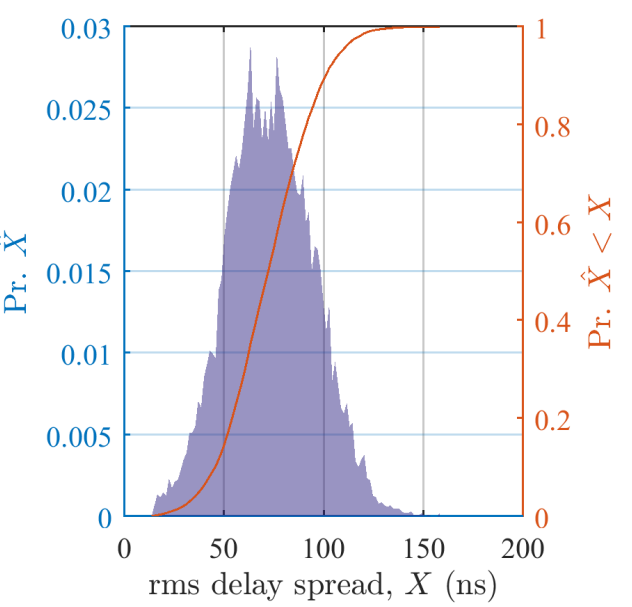

(d) Power Delay Spread

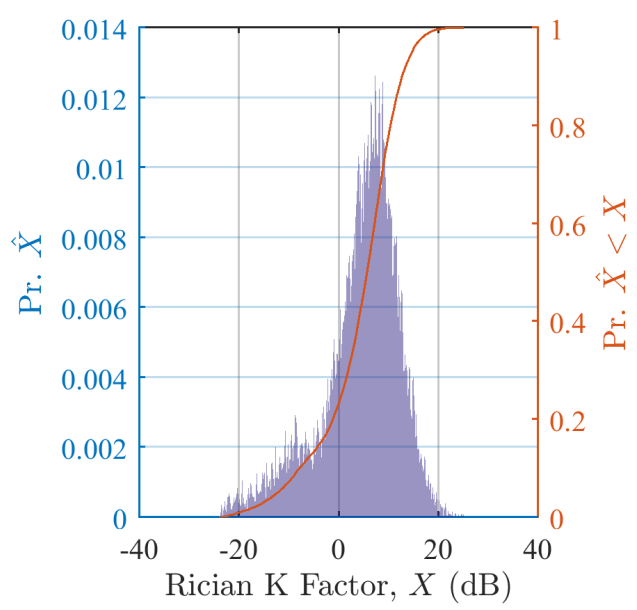

(e) Rician K-factor

Figure A.4.22: Channel characterizations for GBurgD2 5GHz TX1 longPol run4 pp channel stats 


\section{A.4.23 GBurgD2 5GHz TX1 vpol run2 pp.mat}

Table A.69: Measurement Parameters

\begin{tabular}{l|c}
\hline Parameter & Value \\
\hline \hline Mat File & GBurgD2_5GHz_TX1_vpol_run2_pp.mat \\
Frequency $(\mathrm{GHz})$ & 5.400 \\
Location & Machine Shop Day2 at Gaithersburg \\
RX Antenna & Omni-directional, V Pol \\
RX Antenna Gain & -3.500 \\
TX Antenna & Omni-directional, V Pol \\
TX Antenna Gain & 3.600 \\
TX Power, Watts & 1.250 \\
PN Oversample Factor & 4.000 \\
Sample rate, MHz & 80.000
\end{tabular}

Table A.70: Statistics of Channel Estimates. Outliers are removed using a significance test of 0.95.

\begin{tabular}{l||c|c|c|c||c|c||l}
\hline Estimate & $\min (\cdot)$ & $\operatorname{median}(\cdot)$ & $\operatorname{mean}(\cdot)$ & $\max (\cdot)$ & $\operatorname{std}(\cdot)$ & $\operatorname{mad}(\cdot)$ & outlier info \\
\hline \hline$K(\mathrm{~dB})$, NLOS & -23.5 & 10.4 & 9.3 & 28.6 & 8.1 & 6.0 & N: 9746 \\
\hline$K(\mathrm{~dB})$, LOS & 2.3 & 12.8 & 13.2 & 28.6 & 4.2 & 3.5 & N: 6957 \\
\hline$\tau(\mathrm{ns})$ & 8.5 & 129.9 & 135.4 & 349.6 & 65.1 & 53.2 & N: 9746 \\
\hline$S(\mathrm{~ns})$ & 9.8 & 54.6 & 54.7 & 146.3 & 23.2 & 19.1 & N: 9746 \\
\hline
\end{tabular}

Table A.71: Statistics of Channel Estimates. Outliers are not removed. N indicates the total number of samples in the population. Outliers are provided at 10 and 20 times $\sigma$.

\begin{tabular}{l||c|c|c|c||c|c||l}
\hline Estimate & $\min (\cdot)$ & $\operatorname{median}(\cdot)$ & $\operatorname{mean}(\cdot)$ & $\max (\cdot)$ & $\operatorname{std}(\cdot)$ & $\operatorname{mad}(\cdot)$ & outlier info \\
\hline \hline$K(\mathrm{~dB})$, NLOS & -23.5 & 10.4 & 9.3 & 28.6 & 8.1 & 6.0 & N: 9746 \\
\hline$K(\mathrm{~dB})$, LOS & 2.3 & 12.8 & 13.2 & 28.6 & 4.2 & 3.5 & N: 6957 \\
\hline$\tau(\mathrm{ns})$ & 8.5 & 129.9 & 135.4 & 349.6 & 65.1 & 53.2 & N: 9746 \\
\hline$S(\mathrm{~ns})$ & 9.8 & 54.6 & 54.7 & 146.3 & 23.2 & 19.1 & N: 9746 \\
\hline
\end{tabular}




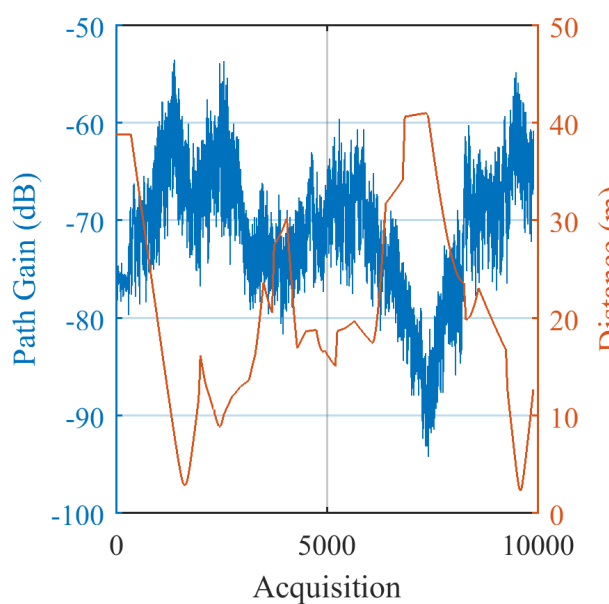

(a) Path Gain versus Acquisition Order

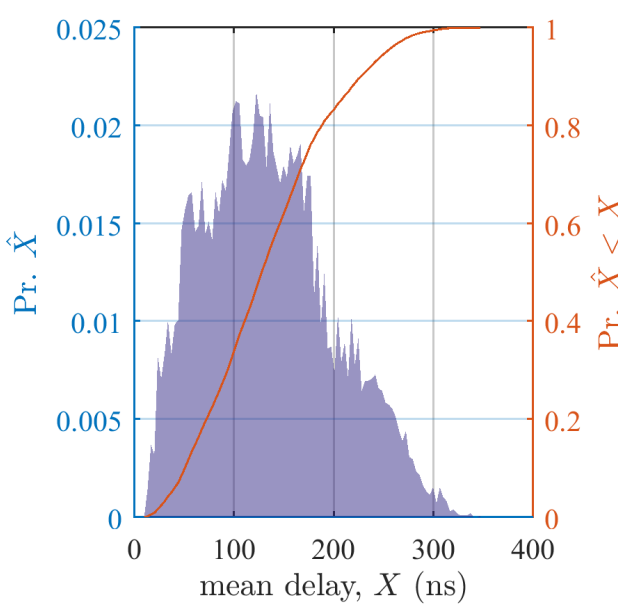

(c) Power Delay

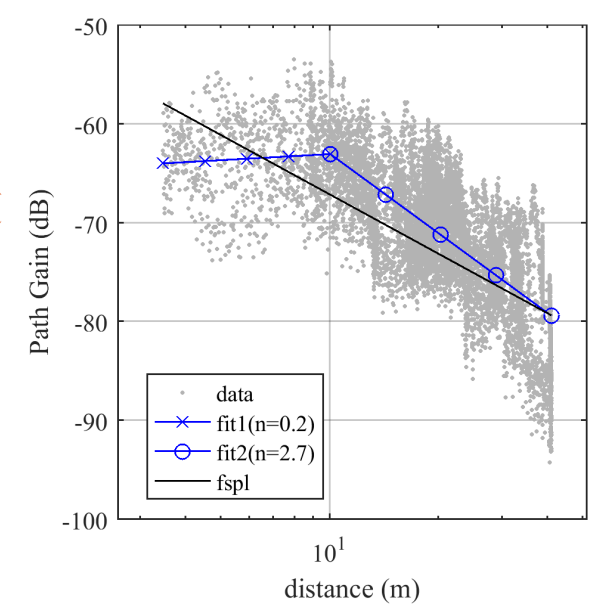

(b) Path Loss versus Distance

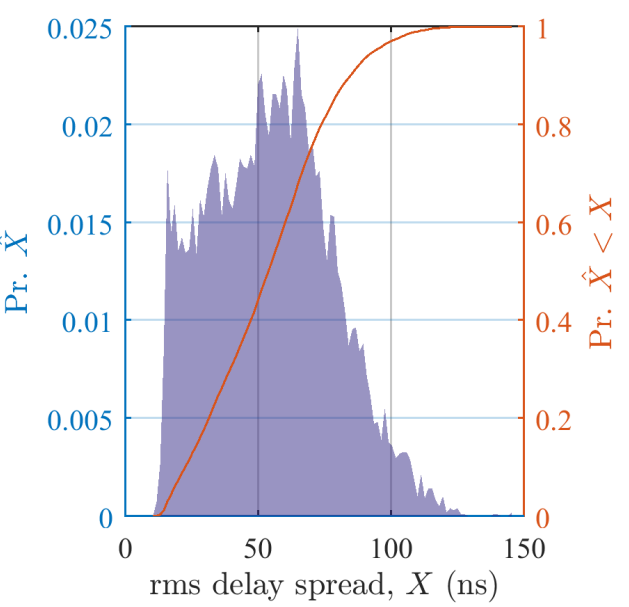

(d) Power Delay Spread

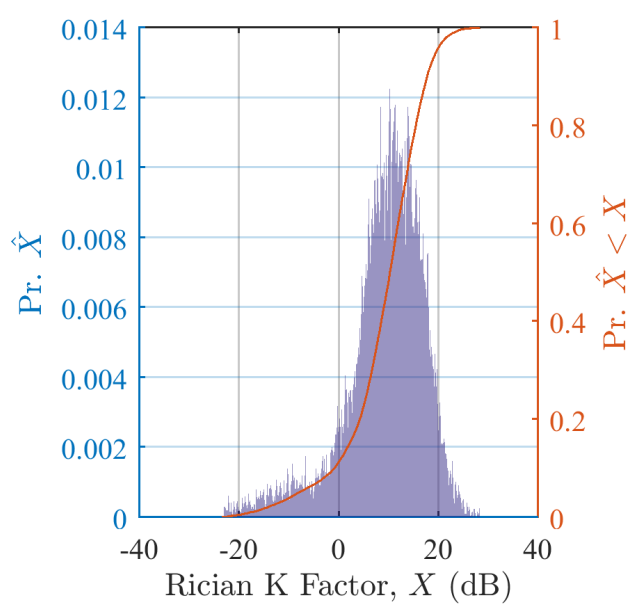

(e) Rician K-factor

Figure A.4.23: Channel characterizations for GBurgD2 5GHz TX1 vpol run2 pp channel stats 


\section{A.4.24 GBurgD2 5GHz TX2high crossPol pp.mat}

Table A.72: Measurement Parameters

\begin{tabular}{l|c}
\hline Parameter & Value \\
\hline \hline Mat File & GBurgD2_5GHz_TX2high_crossPol_pp.mat \\
Frequency (GHz) & 5.400 \\
Location & Machine Shop Day2 at Gaithersburg \\
RX Antenna & Omni-directional, Cross Pol \\
RX Antenna Gain & -3.500 \\
TX Antenna & Omni-directional, V Pol \\
TX Antenna Gain & 3.600 \\
TX Power, Watts & 1.250 \\
PN Oversample Factor & 4.000 \\
Sample rate, MHz & 80.000
\end{tabular}

Table A.73: Statistics of Channel Estimates. Outliers are removed using a significance test of 0.95.

\begin{tabular}{l||c|c|c|c||c|c||l}
\hline Estimate & $\min (\cdot)$ & $\operatorname{median}(\cdot)$ & $\operatorname{mean}(\cdot)$ & $\max (\cdot)$ & $\operatorname{std}(\cdot)$ & $\operatorname{mad}(\cdot)$ & outlier info \\
\hline \hline$K(\mathrm{~dB})$, NLOS & -24.1 & 5.9 & 4.2 & 23.1 & 7.8 & 6.0 & N: 10757 \\
\hline$K(\mathrm{~dB})$, LOS & 2.7 & 10.0 & 10.4 & 22.7 & 2.9 & 2.3 & N: 4872 \\
\hline$\tau(\mathrm{ns})$ & 17.1 & 170.5 & 168.5 & 348.7 & 51.8 & 42.0 & N: 10757 \\
\hline$S(\mathrm{~ns})$ & 12.8 & 78.3 & 78.3 & 167.1 & 20.0 & 16.0 & N: 10757 \\
\hline
\end{tabular}

Table A.74: Statistics of Channel Estimates. Outliers are not removed. N indicates the total number of samples in the population. Outliers are provided at 10 and 20 times $\sigma$.

\begin{tabular}{l||c|c|c|c||c|c||l}
\hline Estimate & $\min (\cdot)$ & $\operatorname{median}(\cdot)$ & $\operatorname{mean}(\cdot)$ & $\max (\cdot)$ & $\operatorname{std}(\cdot)$ & $\operatorname{mad}(\cdot)$ & outlier info \\
\hline \hline$K(\mathrm{~dB})$, NLOS & -24.1 & 5.9 & 4.2 & 23.1 & 7.8 & 6.0 & N: 10757 \\
\hline$K(\mathrm{~dB})$, LOS & 2.7 & 10.0 & 10.4 & 23.1 & 2.9 & 2.3 & N: 4873 \\
\hline$\tau(\mathrm{ns})$ & 17.1 & 170.5 & 168.5 & 348.7 & 51.8 & 42.0 & N: 10757 \\
\hline$S(\mathrm{~ns})$ & 12.8 & 78.3 & 78.3 & 167.1 & 20.0 & 16.0 & N: 10757 \\
\hline
\end{tabular}




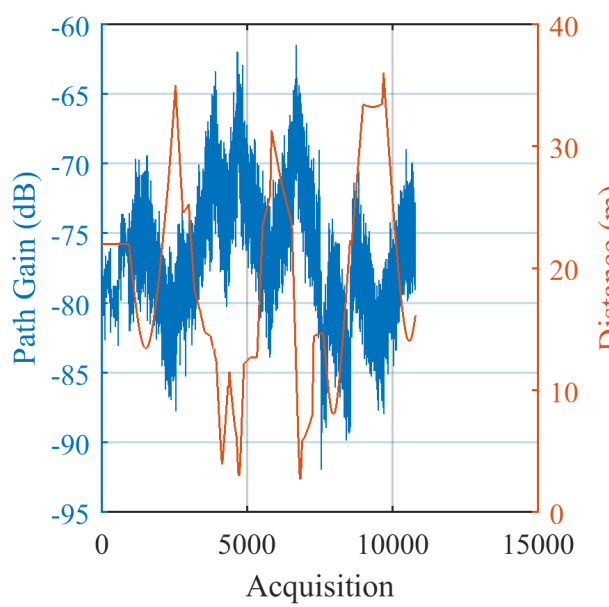

(a) Path Gain versus Acquisition Order

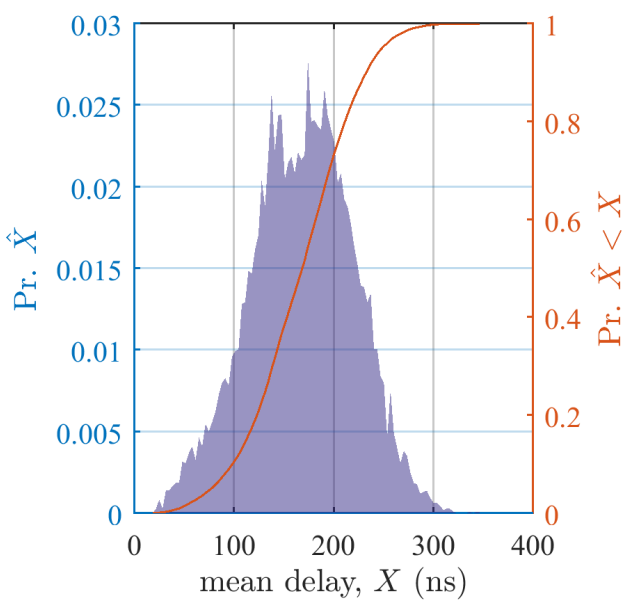

(c) Power Delay

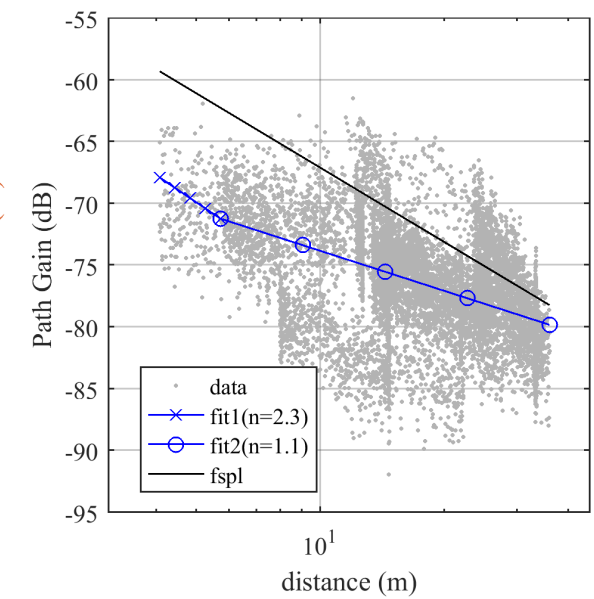

(b) Path Loss versus Distance

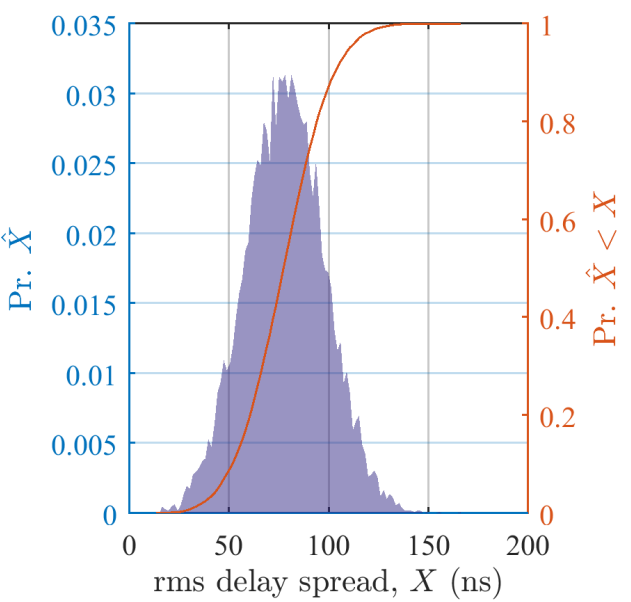

(d) Power Delay Spread

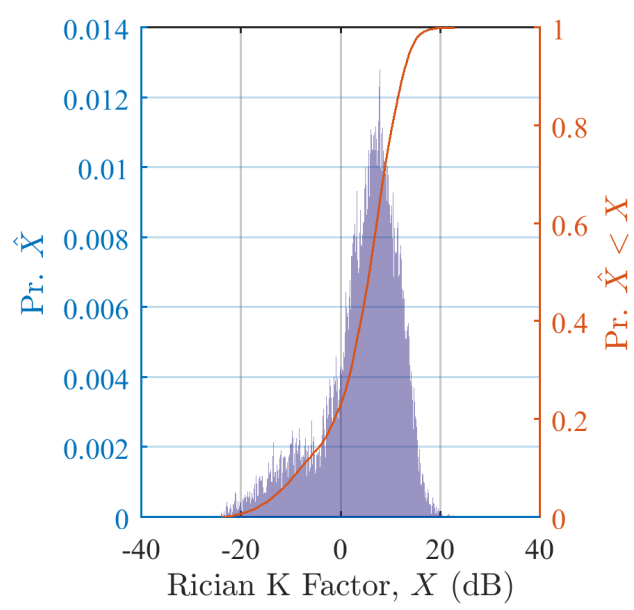

(e) Rician K-factor

Figure A.4.24: Channel characterizations for GBurgD2 5GHz TX2high crossPol pp channel stats 


\section{A.4.25 GBurgD2 5GHz TX2high longPol pp.mat}

Table A.75: Measurement Parameters

\begin{tabular}{l|c}
\hline Parameter & Value \\
\hline \hline Mat File & GBurgD2_5GHz_TX2high_longPol_pp.mat \\
Frequency $(\mathrm{GHz})$ & 5.400 \\
Location & Machine Shop Day2 at Gaithersburg \\
RX Antenna & Omni-directional, Long Pol \\
RX Antenna Gain & -3.500 \\
TX Antenna & Omni-directional, V Pol \\
TX Antenna Gain & 3.600 \\
TX Power, Watts & 1.250 \\
PN Oversample Factor & 4.000 \\
Sample rate, MHz & 80.000
\end{tabular}

Table A.76: Statistics of Channel Estimates. Outliers are removed using a significance test of 0.95.

\begin{tabular}{l||c|c|c|c||c|c||l}
\hline Estimate & $\min (\cdot)$ & $\operatorname{median}(\cdot)$ & $\operatorname{mean}(\cdot)$ & $\max (\cdot)$ & $\operatorname{std}(\cdot)$ & $\operatorname{mad}(\cdot)$ & outlier info \\
\hline \hline$K(\mathrm{~dB})$, NLOS & -24.4 & 6.0 & 4.4 & 24.9 & 7.7 & 5.8 & N: 11382 \\
\hline$K(\mathrm{~dB})$, LOS & 3.6 & 9.8 & 10.2 & 22.4 & 2.9 & 2.3 & N: 5308 \\
\hline$\tau(\mathrm{ns})$ & 30.9 & 169.9 & 170.8 & 340.5 & 52.1 & 41.7 & N: 11382 \\
\hline$S(\mathrm{~ns})$ & 15.7 & 76.9 & 77.4 & 161.1 & 20.6 & 16.5 & N: 11382 \\
\hline
\end{tabular}

Table A.77: Statistics of Channel Estimates. Outliers are not removed. N indicates the total number of samples in the population. Outliers are provided at 10 and 20 times $\sigma$.

\begin{tabular}{l||c|c|c|c||c|c||l}
\hline Estimate & $\min (\cdot)$ & $\operatorname{median}(\cdot)$ & $\operatorname{mean}(\cdot)$ & $\max (\cdot)$ & $\operatorname{std}(\cdot)$ & $\operatorname{mad}(\cdot)$ & outlier info \\
\hline \hline$K(\mathrm{~dB})$, NLOS & -24.4 & 6.0 & 4.4 & 24.9 & 7.7 & 5.8 & N: 11382 \\
\hline$K(\mathrm{~dB})$, LOS & 3.6 & 9.8 & 10.2 & 24.9 & 2.9 & 2.3 & N: 5309 \\
\hline$\tau(\mathrm{ns})$ & 30.9 & 169.9 & 170.8 & 340.5 & 52.1 & 41.7 & N: 11382 \\
\hline$S(\mathrm{~ns})$ & 15.7 & 76.9 & 77.4 & 161.1 & 20.6 & 16.5 & N: 11382 \\
\hline
\end{tabular}




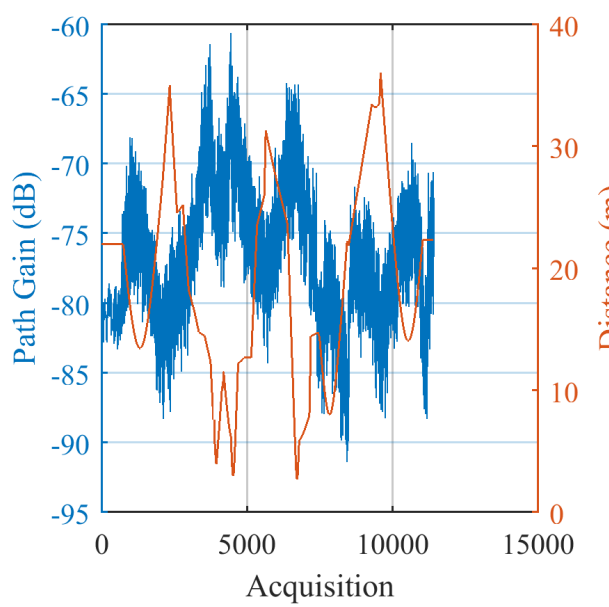

(a) Path Gain versus Acquisition Order

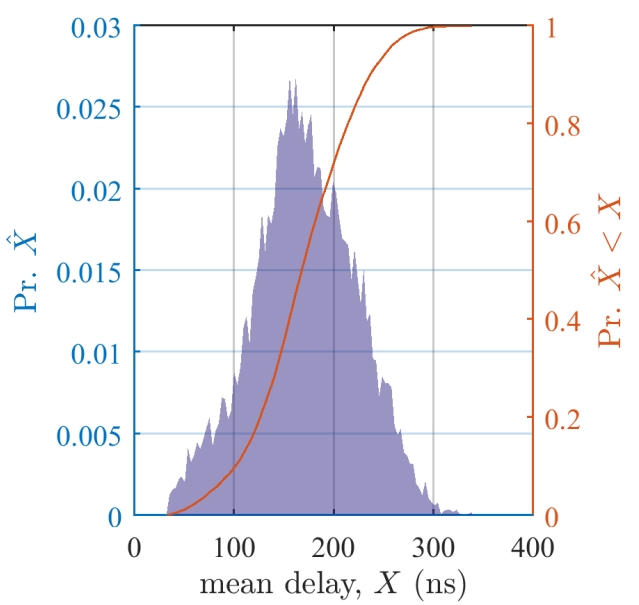

(c) Power Delay

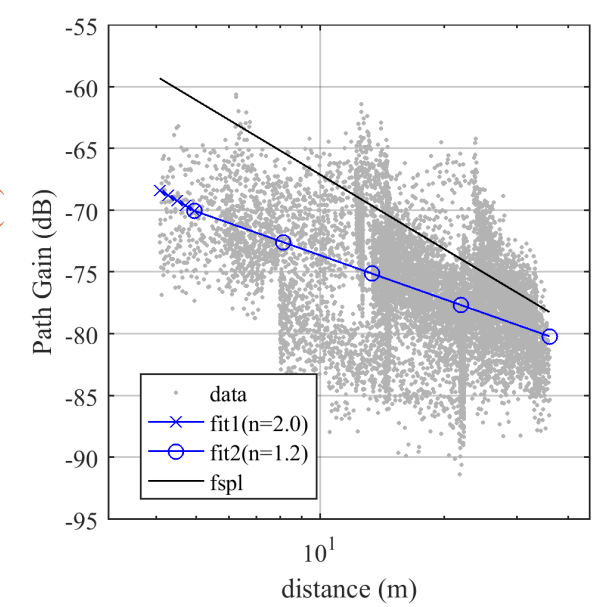

(b) Path Loss versus Distance

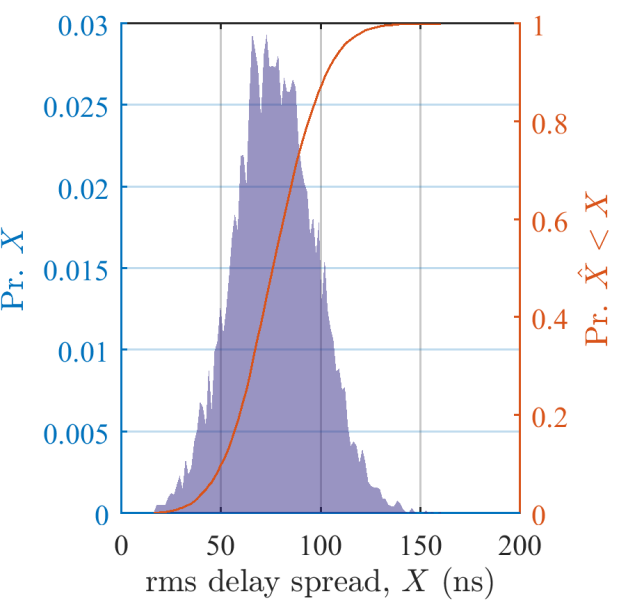

(d) Power Delay Spread

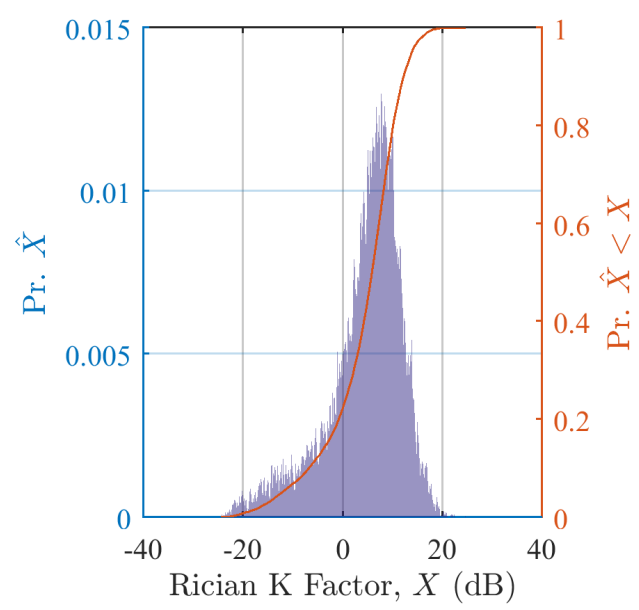

(e) Rician K-factor

Figure A.4.25: Channel characterizations for GBurgD2 5GHz TX2high longPol pp channel stats 


\section{A.4.26 GBurgD2 5GHz TX2low crossPol pp.mat}

Table A.78: Measurement Parameters

\begin{tabular}{l|c}
\hline Parameter & Value \\
\hline \hline Mat File & GBurgD2_5GHz_TX2low_crossPol_pp.mat \\
Frequency $(\mathrm{GHz})$ & 5.400 \\
Location & Machine Shop Day2 at Gaithersburg \\
RX Antenna & Omni-directional, Cross Pol \\
RX Antenna Gain & -3.500 \\
TX Antenna & Omni-directional, V Pol \\
TX Antenna Gain & 3.600 \\
TX Power, Watts & 1.250 \\
PN Oversample Factor & 4.000 \\
Sample rate, MHz & 80.000
\end{tabular}

Table A.79: Statistics of Channel Estimates. Outliers are removed using a significance test of 0.95.

\begin{tabular}{l||c|c|c|c||c|c||l}
\hline Estimate & $\min (\cdot)$ & $\operatorname{median}(\cdot)$ & $\operatorname{mean}(\cdot)$ & $\max (\cdot)$ & $\operatorname{std}(\cdot)$ & $\operatorname{mad}(\cdot)$ & outlier info \\
\hline \hline$K(\mathrm{~dB})$, NLOS & -24.9 & 5.1 & 3.8 & 21.8 & 7.7 & 5.8 & N: 10463 \\
\hline$K(\mathrm{~dB})$, LOS & 3.1 & 9.9 & 10.3 & 21.8 & 3.0 & 2.4 & N: 4283 \\
\hline$\tau(\mathrm{ns})$ & 1478.3 & 1641.8 & 1635.9 & 1797.0 & 54.1 & 43.6 & N: 10463 \\
\hline$S(\mathrm{~ns})$ & 15.2 & 75.1 & 76.6 & 158.5 & 20.4 & 16.3 & N: 10463 \\
\hline
\end{tabular}

Table A.80: Statistics of Channel Estimates. Outliers are not removed. N indicates the total number of samples in the population. Outliers are provided at 10 and 20 times $\sigma$.

\begin{tabular}{l||c|c|c|c||c|c||l}
\hline Estimate & $\min (\cdot)$ & $\operatorname{median}(\cdot)$ & $\operatorname{mean}(\cdot)$ & $\max (\cdot)$ & $\operatorname{std}(\cdot)$ & $\operatorname{mad}(\cdot)$ & outlier info \\
\hline \hline$K(\mathrm{~dB})$, NLOS & -24.9 & 5.1 & 3.8 & 21.8 & 7.7 & 5.8 & N: 10463 \\
\hline$K(\mathrm{~dB})$, LOS & 3.1 & 9.9 & 10.3 & 21.8 & 3.0 & 2.4 & N: 4283 \\
\hline$\tau(\mathrm{ns})$ & 1478.3 & 1641.8 & 1635.9 & 1797.0 & 54.1 & 43.6 & N: 10463 \\
\hline$S(\mathrm{~ns})$ & 15.2 & 75.1 & 76.6 & 158.5 & 20.4 & 16.3 & N: 10463 \\
\hline
\end{tabular}




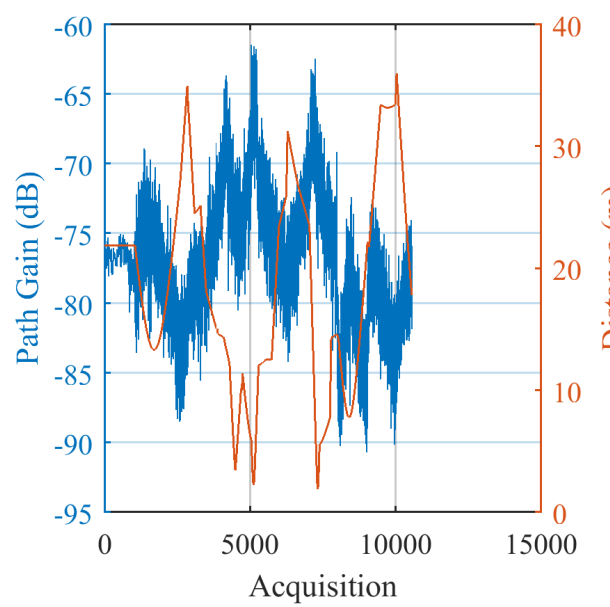

(a) Path Gain versus Acquisition Order

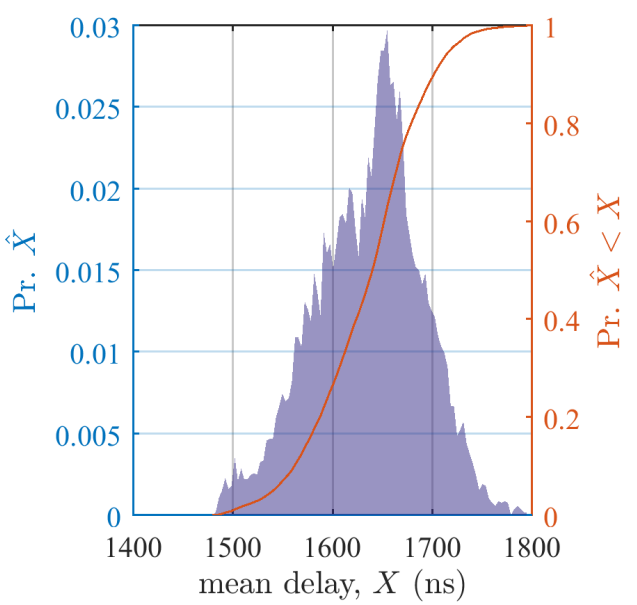

(c) Power Delay

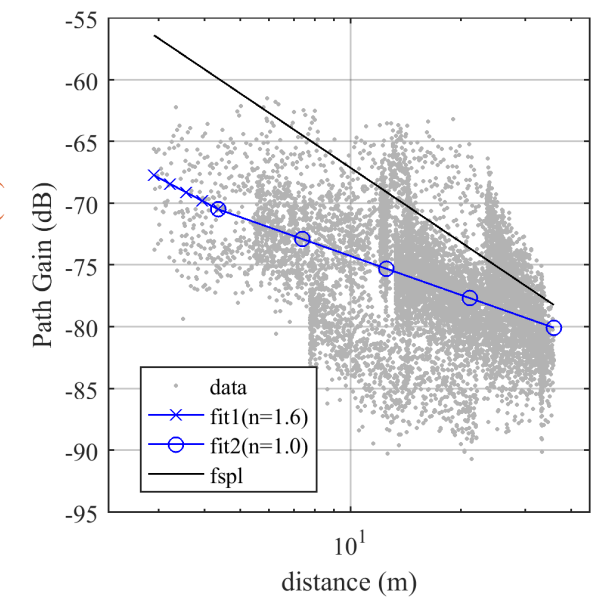

(b) Path Loss versus Distance

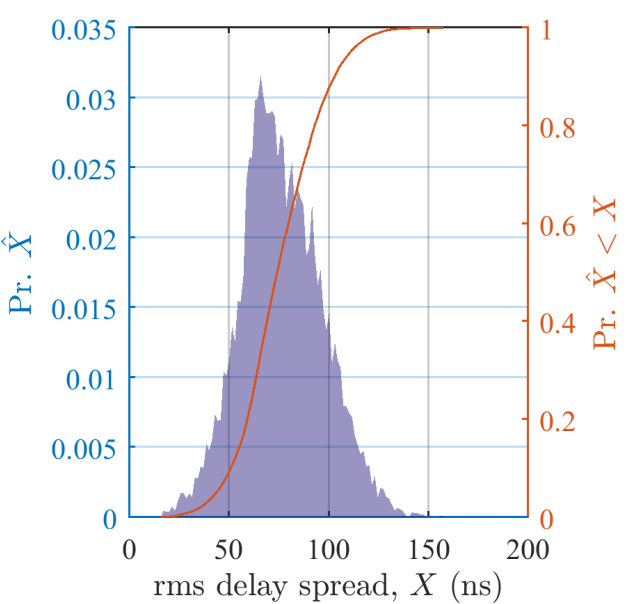

(d) Power Delay Spread

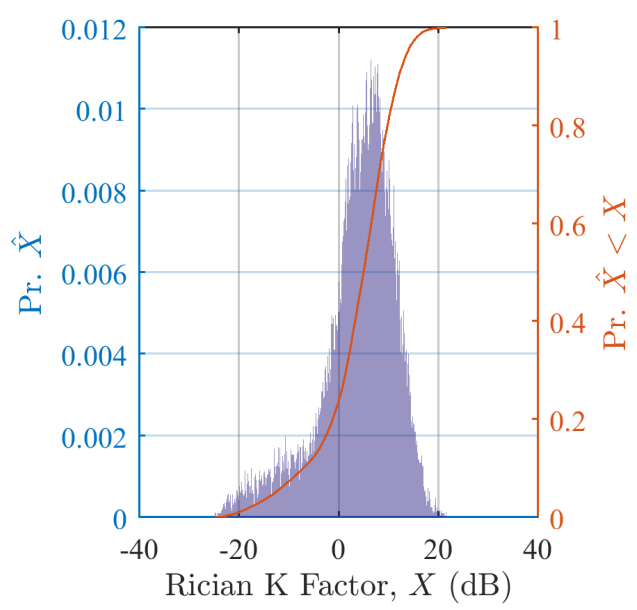

(e) Rician K-factor

Figure A.4.26: Channel characterizations for GBurgD2 5GHz TX2low crossPol pp channel stats 


\section{A.4.27 GBurgD2 5GHz TX2low longPol pp.mat}

Table A.81: Measurement Parameters

\begin{tabular}{l|c}
\hline Parameter & Value \\
\hline \hline Mat File & GBurgD2_5GHz_TX2low_longPol_pp.mat \\
Frequency $(\mathrm{GHz})$ & 5.400 \\
Location & Machine Shop Day2 at Gaithersburg \\
RX Antenna & Omni-directional, Long Pol \\
RX Antenna Gain & -3.500 \\
TX Antenna & Omni-directional, V Pol \\
TX Antenna Gain & 3.600 \\
TX Power, Watts & 1.250 \\
PN Oversample Factor & 4.000 \\
Sample rate, MHz & 80.000
\end{tabular}

Table A.82: Statistics of Channel Estimates. Outliers are removed using a significance test of 0.95.

\begin{tabular}{l||c|c|c|c||c|c||l}
\hline Estimate & $\min (\cdot)$ & $\operatorname{median}(\cdot)$ & $\operatorname{mean}(\cdot)$ & $\max (\cdot)$ & $\operatorname{std}(\cdot)$ & $\operatorname{mad}(\cdot)$ & outlier info \\
\hline \hline$K(\mathrm{~dB})$, NLOS & -14.8 & 2.7 & 2.4 & 14.9 & 4.9 & 3.9 & $\mathrm{~N}: 9903$ \\
\hline$K(\mathrm{~dB})$, LOS & 0.0 & 5.9 & 6.2 & 14.9 & 2.8 & 2.2 & $\mathrm{~N}: 4821$ \\
\hline$\tau(\mathrm{ns})$ & 70008.7 & 70164.5 & 70162.6 & 70344.9 & 54.0 & 43.9 & $\mathrm{~N}: 9892$ \\
\hline$S(\mathrm{~ns})$ & 0.0 & 72.0 & 71.7 & 149.9 & 23.8 & 18.3 & $\mathrm{~N}: 10147$ \\
\hline
\end{tabular}

Table A.83: Statistics of Channel Estimates. Outliers are not removed. N indicates the total number of samples in the population. Outliers are provided at 10 and 20 times $\sigma$.

\begin{tabular}{l||c|c|c|c||c|c||l}
\hline Estimate & $\min (\cdot)$ & $\operatorname{median}(\cdot)$ & $\operatorname{mean}(\cdot)$ & $\max (\cdot)$ & $\operatorname{std}(\cdot)$ & $\operatorname{mad}(\cdot)$ & outlier info \\
\hline \hline$K(\mathrm{~dB})$, NLOS & -14.8 & 2.7 & 2.4 & 14.9 & 4.9 & 3.9 & $\mathrm{~N}: 9903$ \\
\hline$K(\mathrm{~dB})$, LOS & 0.0 & 5.9 & 6.2 & 14.9 & 2.8 & 2.2 & $\mathrm{~N}: 4821$ \\
\hline$\tau(\mathrm{ns})$ & 212.5 & 70162.9 & 69202.8 & 81425.0 & 6749.5 & 1901.2 & $\mathrm{~N}: 10197$ \\
\hline$S(\mathrm{~ns})$ & 0.0 & 72.2 & 134.7 & 36466.4 & 1095.1 & 125.4 & $\mathrm{~N}: 10197$ \\
& & & & & & & $10 \sigma(11085.8): 24$ \\
& & & & & & & $20 \sigma(22036.9): 9$ \\
\hline
\end{tabular}




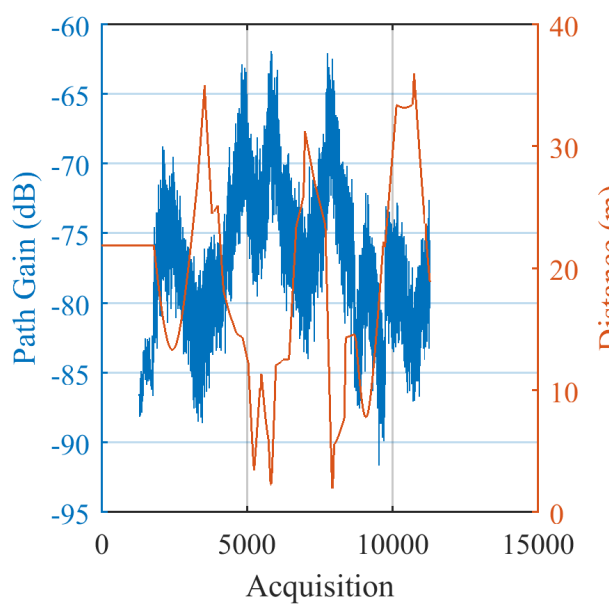

(a) Path Gain versus Acquisition Order

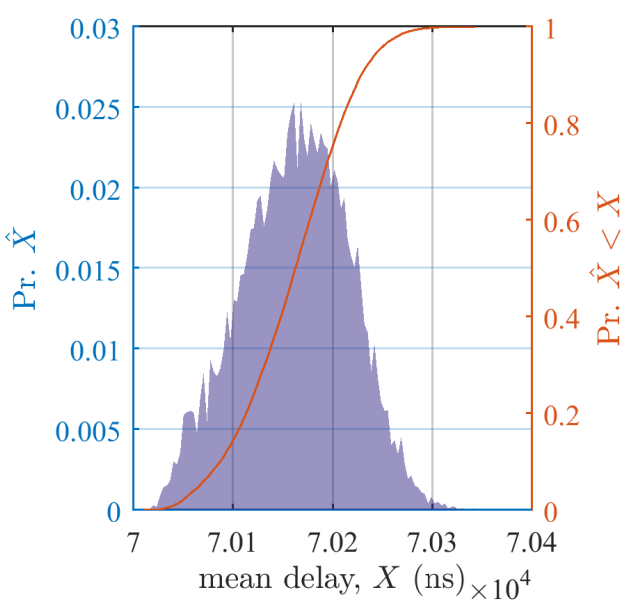

(c) Power Delay

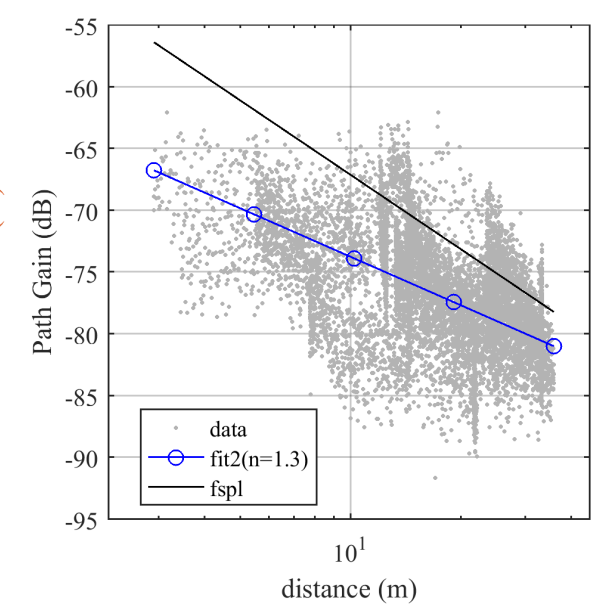

(b) Path Loss versus Distance

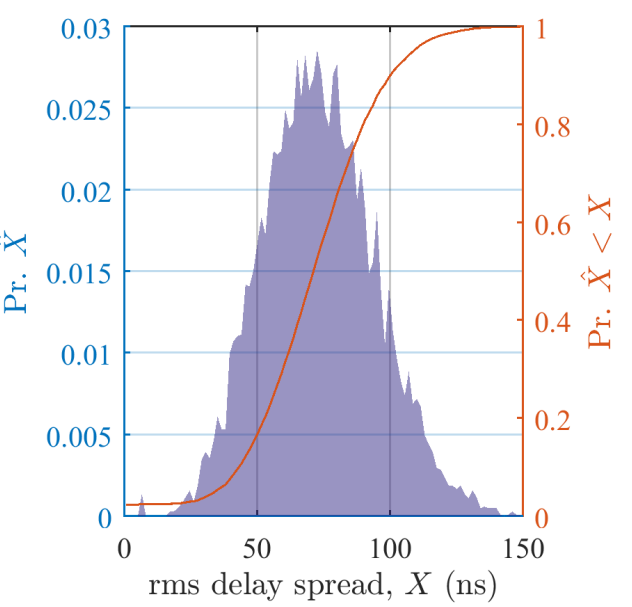

(d) Power Delay Spread

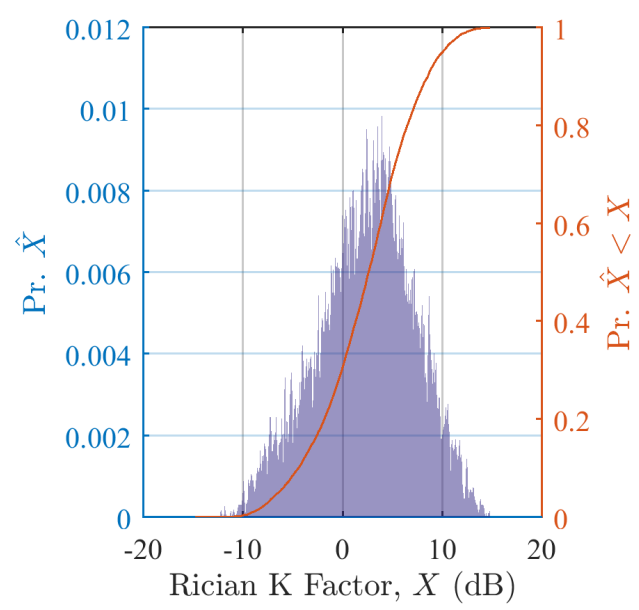

(e) Rician K-factor

Figure A.4.27: Channel characterizations for GBurgD2 5GHz TX2low longPol pp channel stats 


\section{A.4.28 GBurgD2 5GHz TX2low vpol run1 pp.mat}

Table A.84: Measurement Parameters

\begin{tabular}{l|c}
\hline Parameter & Value \\
\hline \hline Mat File & GBurgD2_5GHz_TX2low_vpol_run1_pp.mat \\
Frequency (GHz) & 5.400 \\
Location & Machine Shop Day2 at Gaithersburg \\
RX Antenna & Omni-directional, V Pol \\
RX Antenna Gain & -3.500 \\
TX Antenna & Omni-directional, V Pol \\
TX Antenna Gain & 3.600 \\
TX Power, Watts & 1.250 \\
PN Oversample Factor & 4.000 \\
Sample rate, MHz & 80.000
\end{tabular}

Table A.85: Statistics of Channel Estimates. Outliers are removed using a significance test of 0.95.

\begin{tabular}{l||c|c|c|c||c|c||l}
\hline Estimate & $\min (\cdot)$ & $\operatorname{median}(\cdot)$ & $\operatorname{mean}(\cdot)$ & $\max (\cdot)$ & $\operatorname{std}(\cdot)$ & $\operatorname{mad}(\cdot)$ & outlier info \\
\hline \hline$K(\mathrm{~dB})$, NLOS & -21.9 & 9.9 & 8.9 & 29.1 & 6.9 & 5.1 & N: 9479 \\
\hline$K(\mathrm{~dB})$, LOS & 3.4 & 11.9 & 12.2 & 27.2 & 3.7 & 3.0 & N: 6785 \\
\hline$\tau(\mathrm{ns})$ & 14.2 & 115.9 & 118.9 & 304.7 & 53.3 & 44.0 & N: 9481 \\
\hline$S(\mathrm{~ns})$ & 12.4 & 55.1 & 56.9 & 146.4 & 21.5 & 17.3 & N: 9481 \\
\hline
\end{tabular}

Table A.86: Statistics of Channel Estimates. Outliers are not removed. N indicates the total number of samples in the population. Outliers are provided at 10 and 20 times $\sigma$.

\begin{tabular}{l||c|c|c|c||c|c||l}
\hline Estimate & $\min (\cdot)$ & $\operatorname{median}(\cdot)$ & $\operatorname{mean}(\cdot)$ & $\max (\cdot)$ & $\operatorname{std}(\cdot)$ & $\operatorname{mad}(\cdot)$ & outlier info \\
\hline \hline$K(\mathrm{~dB})$, NLOS & -23.7 & 9.9 & 8.9 & 29.1 & 6.9 & 5.1 & N: 9481 \\
\hline$K(\mathrm{~dB})$, LOS & 3.4 & 11.9 & 12.2 & 29.1 & 3.7 & 3.0 & N: 6786 \\
\hline$\tau(\mathrm{ns})$ & 14.2 & 115.9 & 118.9 & 304.7 & 53.3 & 44.0 & N: 9481 \\
\hline$S(\mathrm{~ns})$ & 12.4 & 55.1 & 56.9 & 146.4 & 21.5 & 17.3 & N: 9481 \\
\hline
\end{tabular}




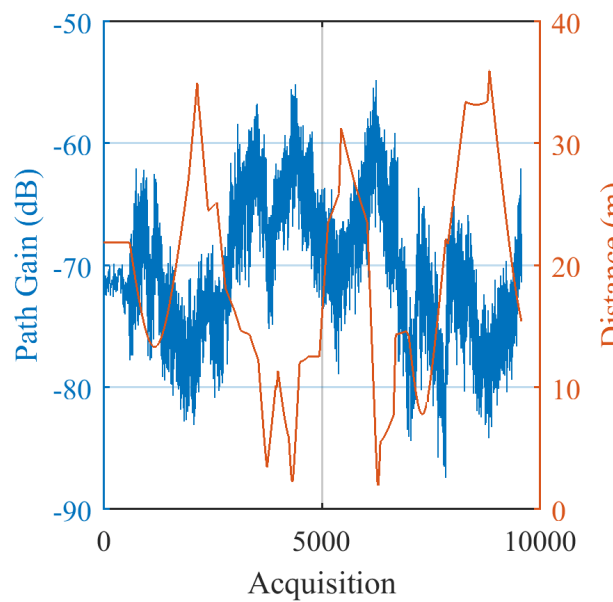

(a) Path Gain versus Acquisition Order

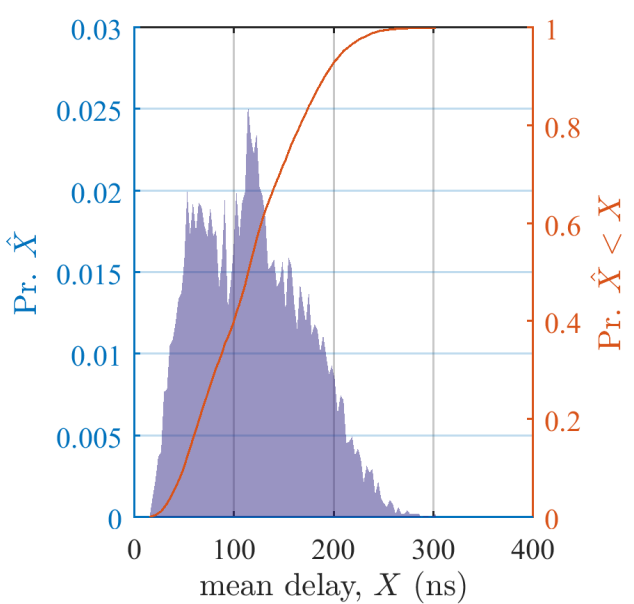

(c) Power Delay

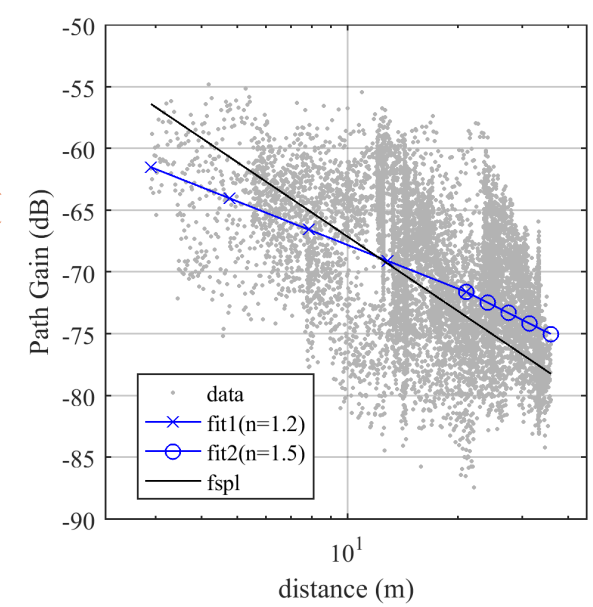

(b) Path Loss versus Distance

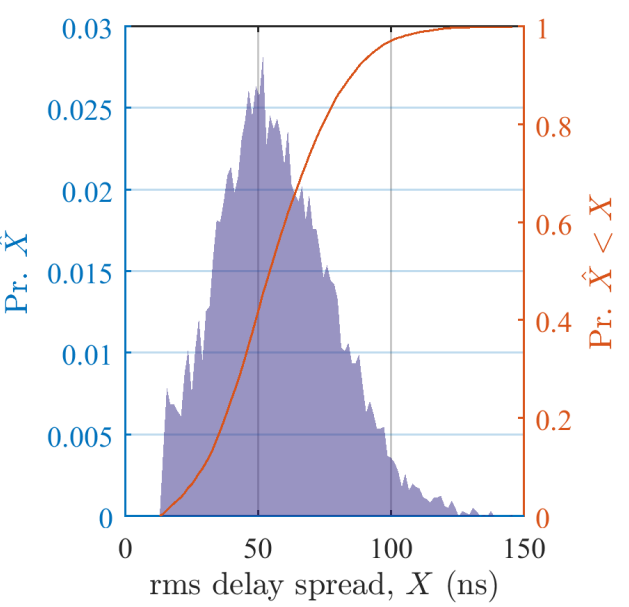

(d) Power Delay Spread

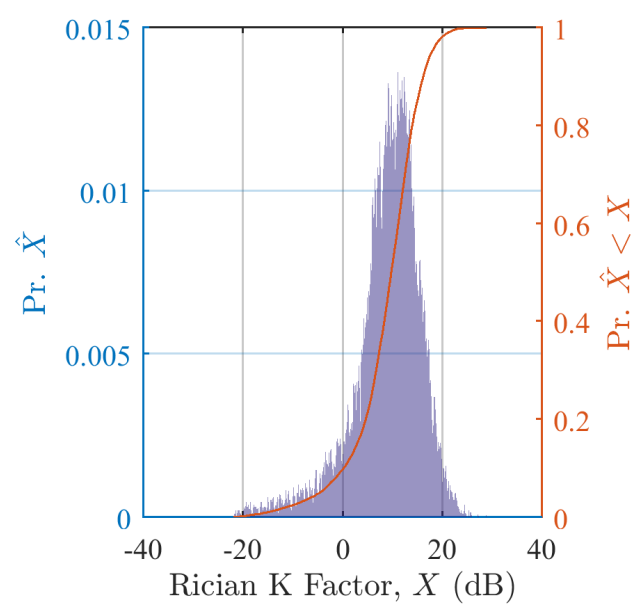

(e) Rician K-factor

Figure A.4.28: Channel characterizations for GBurgD2 5GHz TX2low vpol run1 pp channel stats 


\section{A.4.29 GBurgD3 2GHz TX2high crossPol run2 pp.mat}

Table A.87: Measurement Parameters

\begin{tabular}{l|c}
\hline Parameter & Value \\
\hline \hline Mat File & GBurgD3_2GHz_TX2high_crossPol_run2_pp.mat \\
Frequency (GHz) & 2.245 \\
Location & Machine Shop Day3 at Gaithersburg \\
RX Antenna & Omni-directional, Cross Pol \\
RX Antenna Gain & -4.200 \\
TX Antenna & Omni-directional, V Pol \\
TX Antenna Gain & 2.900 \\
TX Power, Watts & 1.500 \\
PN Oversample Factor & 4.000 \\
Sample rate, MHz & 80.000
\end{tabular}

Table A.88: Statistics of Channel Estimates. Outliers are removed using a significance test of 0.95.

\begin{tabular}{l||c|c|c|c||c|c||l}
\hline Estimate & $\min (\cdot)$ & $\operatorname{median}(\cdot)$ & $\operatorname{mean}(\cdot)$ & $\max (\cdot)$ & $\operatorname{std}(\cdot)$ & $\operatorname{mad}(\cdot)$ & outlier info \\
\hline \hline$K(\mathrm{~dB})$, NLOS & -24.9 & 6.7 & 5.1 & 22.9 & 7.9 & 5.9 & N: 10082 \\
\hline$K(\mathrm{~dB})$, LOS & 3.6 & 10.5 & 10.8 & 22.9 & 3.0 & 2.5 & N: 5041 \\
\hline$\tau(\mathrm{ns})$ & 29.2 & 182.0 & 186.9 & 382.0 & 59.3 & 49.3 & N: 10082 \\
\hline$S(\mathrm{~ns})$ & 14.4 & 79.0 & 79.3 & 157.0 & 22.3 & 17.8 & N: 10082 \\
\hline
\end{tabular}

Table A.89: Statistics of Channel Estimates. Outliers are not removed. N indicates the total number of samples in the population. Outliers are provided at 10 and 20 times $\sigma$.

\begin{tabular}{l||c|c|c|c||c|c||l}
\hline Estimate & $\min (\cdot)$ & $\operatorname{median}(\cdot)$ & $\operatorname{mean}(\cdot)$ & $\max (\cdot)$ & $\operatorname{std}(\cdot)$ & $\operatorname{mad}(\cdot)$ & outlier info \\
\hline \hline$K(\mathrm{~dB})$, NLOS & -24.9 & 6.7 & 5.1 & 22.9 & 7.9 & 5.9 & N: 10082 \\
\hline$K(\mathrm{~dB})$, LOS & 3.6 & 10.5 & 10.8 & 22.9 & 3.0 & 2.5 & N: 5041 \\
\hline$\tau(\mathrm{ns})$ & 29.2 & 182.0 & 186.9 & 382.0 & 59.3 & 49.3 & N: 10082 \\
\hline$S(\mathrm{~ns})$ & 14.4 & 79.0 & 79.3 & 157.0 & 22.3 & 17.8 & N: 10082 \\
\hline
\end{tabular}




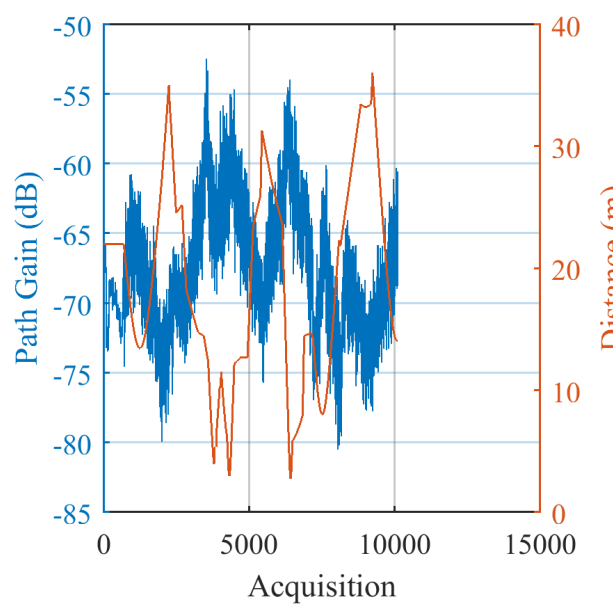

(a) Path Gain versus Acquisition Order

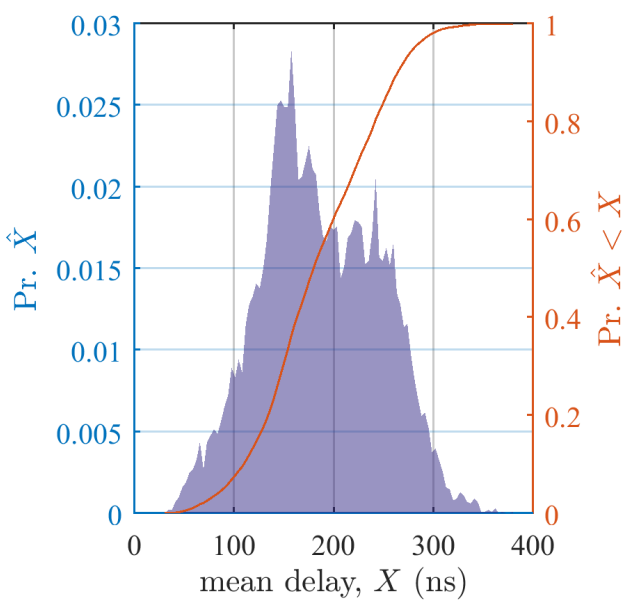

(c) Power Delay

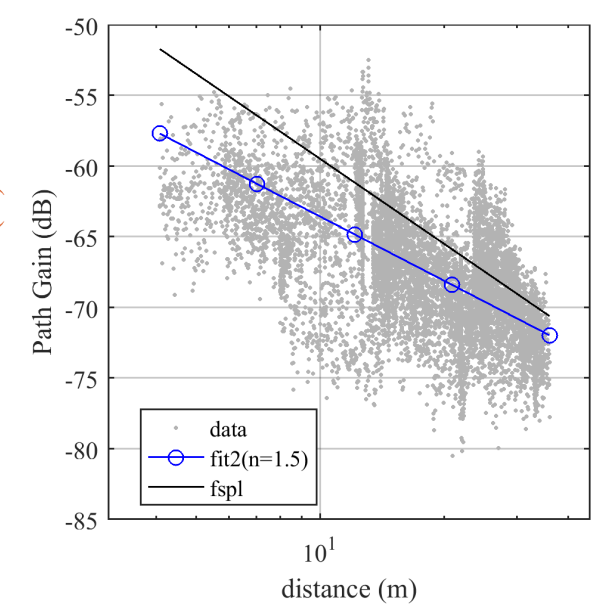

(b) Path Loss versus Distance

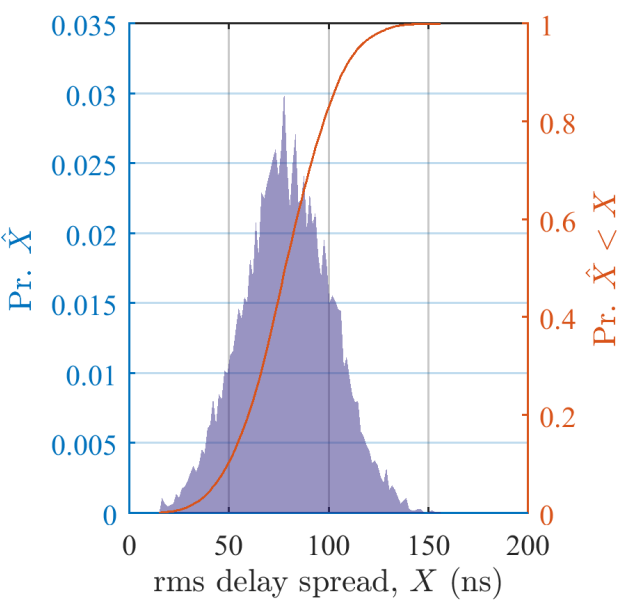

(d) Power Delay Spread

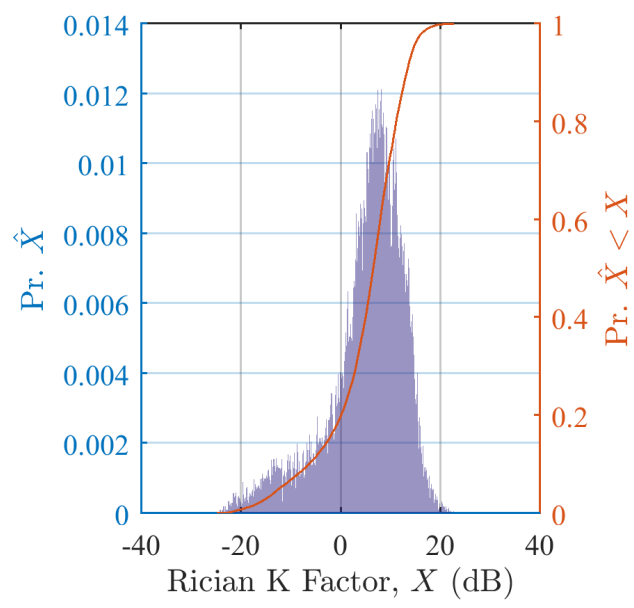

(e) Rician K-factor

Figure A.4.29: Channel characterizations for GBurgD3 2GHz TX2high crossPol run2 pp channel stats 


\section{A.4.30 GBurgD3 2GHz TX2high longPol run3 pp.mat}

Table A.90: Measurement Parameters

\begin{tabular}{l|c}
\hline Parameter & Value \\
\hline \hline Mat File & GBurgD3_2GHz_TX2high_longPol_run3_pp.mat \\
Frequency (GHz) & 2.245 \\
Location & Machine Shop Day3 at Gaithersburg \\
RX Antenna & Omni-directional, Long Pol \\
RX Antenna Gain & -4.200 \\
TX Antenna & Omni-directional, V Pol \\
TX Antenna Gain & 2.900 \\
TX Power, Watts & 1.500 \\
PN Oversample Factor & 4.000 \\
Sample rate, MHz & 80.000
\end{tabular}

Table A.91: Statistics of Channel Estimates. Outliers are removed using a significance test of 0.95.

\begin{tabular}{l||c|c|c|c||c|c||l}
\hline Estimate & $\min (\cdot)$ & $\operatorname{median}(\cdot)$ & $\operatorname{mean}(\cdot)$ & $\max (\cdot)$ & $\operatorname{std}(\cdot)$ & $\operatorname{mad}(\cdot)$ & outlier info \\
\hline \hline$K(\mathrm{~dB})$, NLOS & -24.7 & 6.7 & 4.9 & 24.2 & 7.6 & 5.7 & N: 11117 \\
\hline$K(\mathrm{~dB})$, LOS & 3.7 & 9.8 & 10.3 & 22.0 & 2.8 & 2.2 & N: 5490 \\
\hline$\tau(\mathrm{ns})$ & 33.3 & 181.8 & 184.6 & 376.1 & 60.8 & 49.4 & N: 11117 \\
\hline$S(\mathrm{~ns})$ & 14.9 & 80.2 & 80.2 & 168.8 & 22.0 & 17.3 & N: 11117 \\
\hline
\end{tabular}

Table A.92: Statistics of Channel Estimates. Outliers are not removed. N indicates the total number of samples in the population. Outliers are provided at 10 and 20 times $\sigma$.

\begin{tabular}{l||c|c|c|c||c|c||l}
\hline Estimate & $\min (\cdot)$ & $\operatorname{median}(\cdot)$ & $\operatorname{mean}(\cdot)$ & $\max (\cdot)$ & $\operatorname{std}(\cdot)$ & $\operatorname{mad}(\cdot)$ & outlier info \\
\hline \hline$K(\mathrm{~dB})$, NLOS & -24.7 & 6.7 & 4.9 & 24.2 & 7.6 & 5.7 & N: 11117 \\
\hline$K(\mathrm{~dB})$, LOS & 3.7 & 9.8 & 10.3 & 24.2 & 2.8 & 2.2 & N: 5491 \\
\hline$\tau(\mathrm{ns})$ & 33.3 & 181.8 & 184.6 & 376.1 & 60.8 & 49.4 & N: 11117 \\
\hline$S(\mathrm{~ns})$ & 14.9 & 80.2 & 80.2 & 168.8 & 22.0 & 17.3 & N: 11117 \\
\hline
\end{tabular}




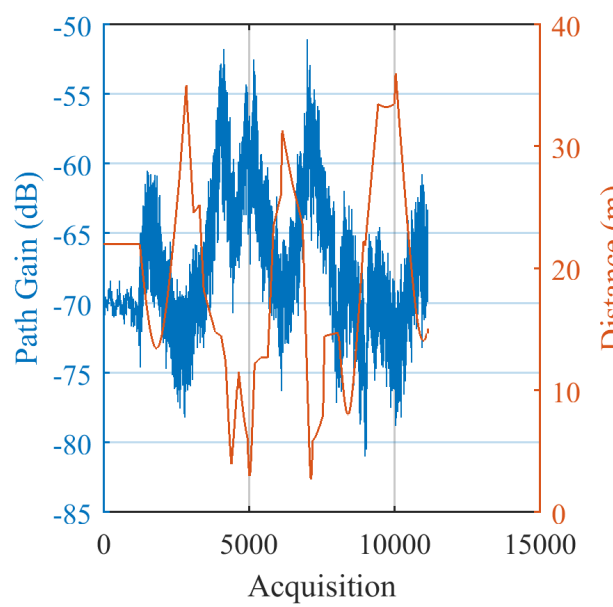

(a) Path Gain versus Acquisition Order

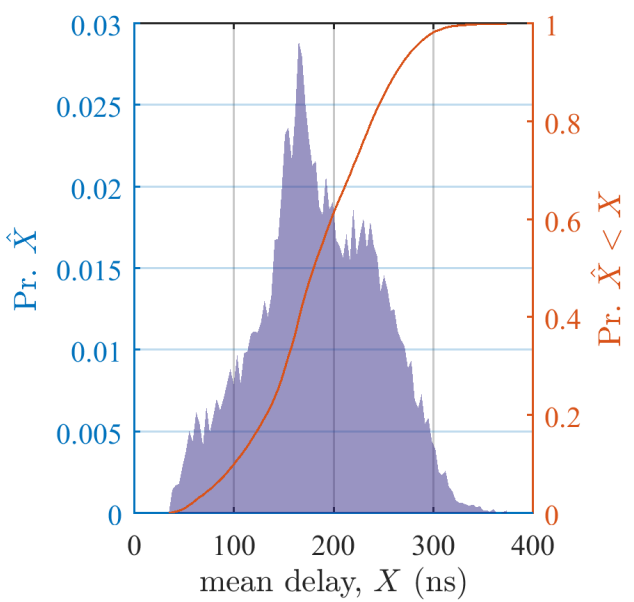

(c) Power Delay

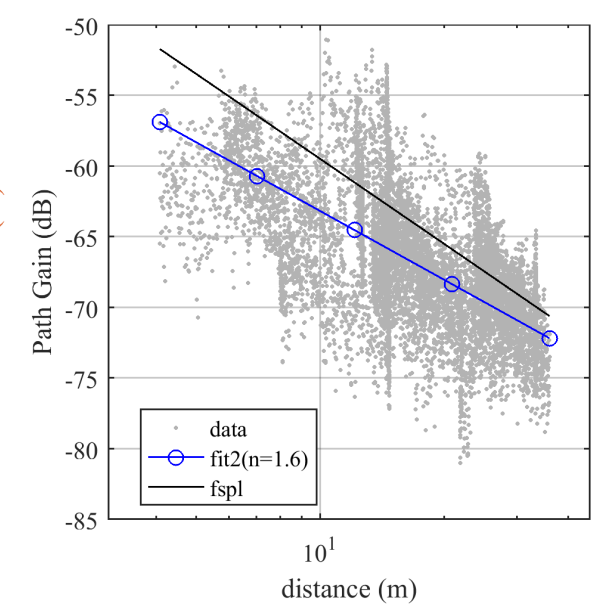

(b) Path Loss versus Distance

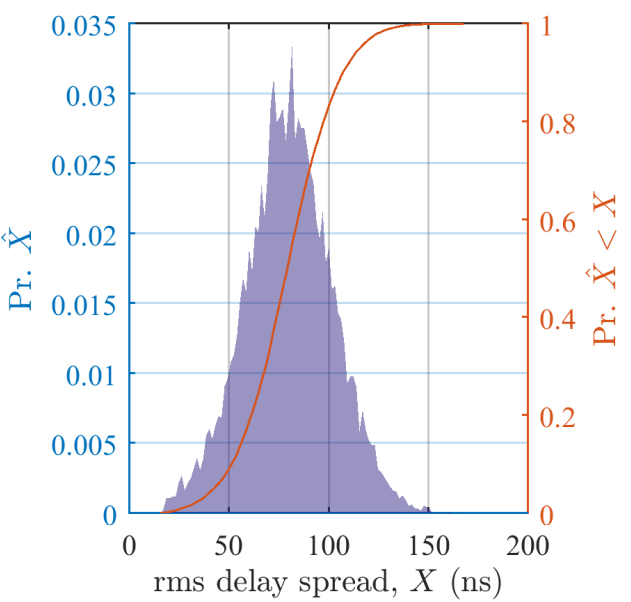

(d) Power Delay Spread

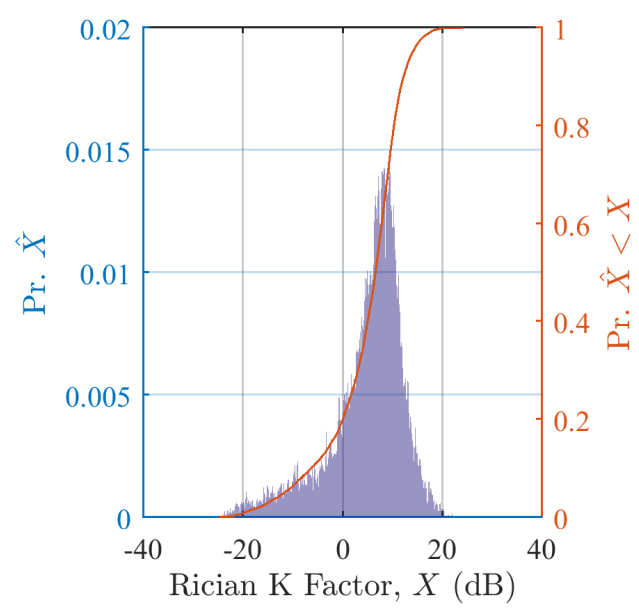

(e) Rician K-factor

Figure A.4.30: Channel characterizations for GBurgD3 2GHz TX2high longPol run3 pp channel stats 


\section{A.4.31 GBurgD3 2GHz TX2low crossPol run2 pp.mat}

Table A.93: Measurement Parameters

\begin{tabular}{l|c}
\hline Parameter & Value \\
\hline \hline Mat File & GBurgD3_2GHz_TX2low_crossPol_run2_pp.mat \\
Frequency (GHz) & 2.245 \\
Location & Machine Shop Day3 at Gaithersburg \\
RX Antenna & Omni-directional, Cross Pol \\
RX Antenna Gain & -4.200 \\
TX Antenna & Omni-directional, V Pol \\
TX Antenna Gain & 2.900 \\
TX Power, Watts & 1.500 \\
PN Oversample Factor & 4.000 \\
Sample rate, MHz & 80.000
\end{tabular}

Table A.94: Statistics of Channel Estimates. Outliers are removed using a significance test of 0.95.

\begin{tabular}{l||c|c|c|c||c|c||l}
\hline Estimate & $\min (\cdot)$ & $\operatorname{median}(\cdot)$ & $\operatorname{mean}(\cdot)$ & $\max (\cdot)$ & $\operatorname{std}(\cdot)$ & $\operatorname{mad}(\cdot)$ & outlier info \\
\hline \hline$K(\mathrm{~dB})$, NLOS & -24.5 & 5.7 & 4.2 & 23.1 & 7.6 & 5.7 & N: 10924 \\
\hline$K(\mathrm{~dB})$, LOS & 3.6 & 9.9 & 10.4 & 23.1 & 3.0 & 2.4 & N: 4361 \\
\hline$\tau(\mathrm{ns})$ & 31.8 & 201.8 & 198.3 & 405.9 & 59.3 & 47.6 & N: 10924 \\
\hline$S(\mathrm{~ns})$ & 14.3 & 82.1 & 81.3 & 160.5 & 21.9 & 17.6 & N: 10924 \\
\hline
\end{tabular}

Table A.95: Statistics of Channel Estimates. Outliers are not removed. N indicates the total number of samples in the population. Outliers are provided at 10 and 20 times $\sigma$.

\begin{tabular}{l||c|c|c|c||c|c||l}
\hline Estimate & $\min (\cdot)$ & $\operatorname{median}(\cdot)$ & $\operatorname{mean}(\cdot)$ & $\max (\cdot)$ & $\operatorname{std}(\cdot)$ & $\operatorname{mad}(\cdot)$ & outlier info \\
\hline \hline$K(\mathrm{~dB})$, NLOS & -24.5 & 5.7 & 4.2 & 23.1 & 7.6 & 5.7 & N: 10924 \\
\hline$K(\mathrm{~dB})$, LOS & 3.6 & 9.9 & 10.4 & 23.1 & 3.0 & 2.4 & N: 4361 \\
\hline$\tau(\mathrm{ns})$ & 31.8 & 201.8 & 198.3 & 405.9 & 59.3 & 47.6 & N: 10924 \\
\hline$S(\mathrm{~ns})$ & 14.3 & 82.1 & 81.3 & 160.5 & 21.9 & 17.6 & N: 10924 \\
\hline
\end{tabular}




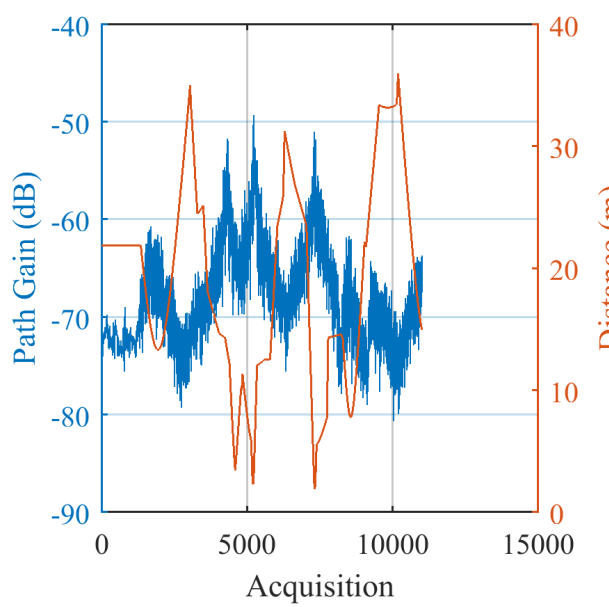

(a) Path Gain versus Acquisition Order

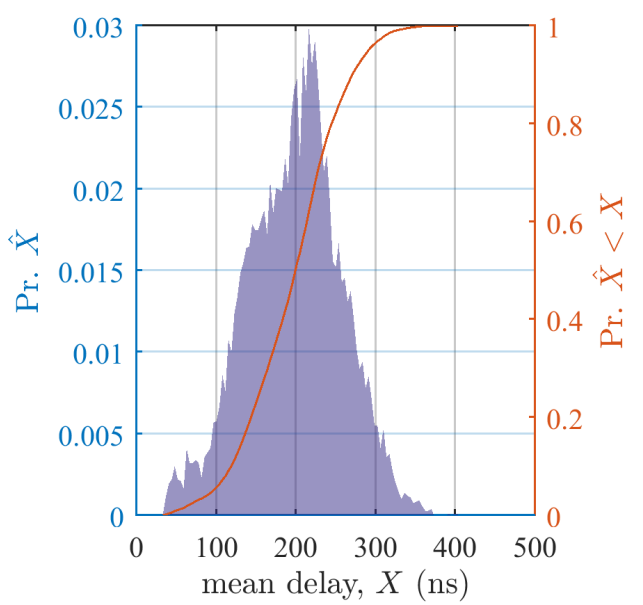

(c) Power Delay

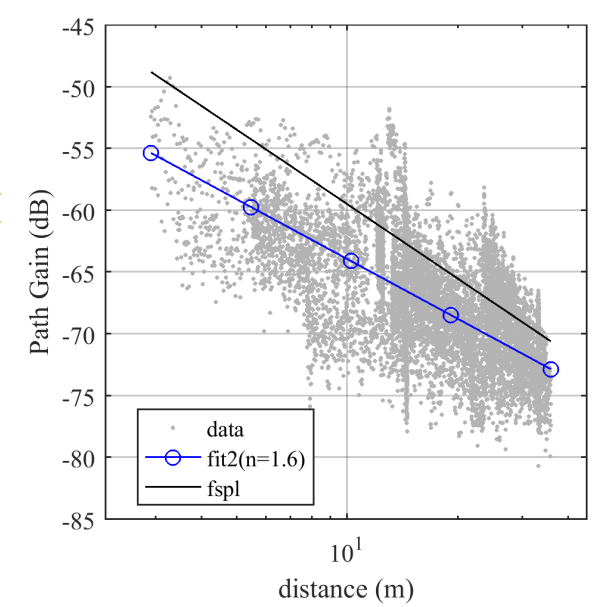

(b) Path Loss versus Distance

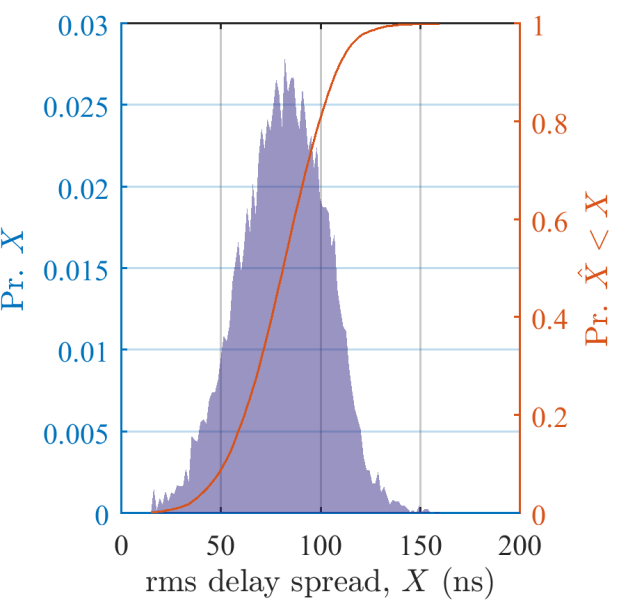

(d) Power Delay Spread

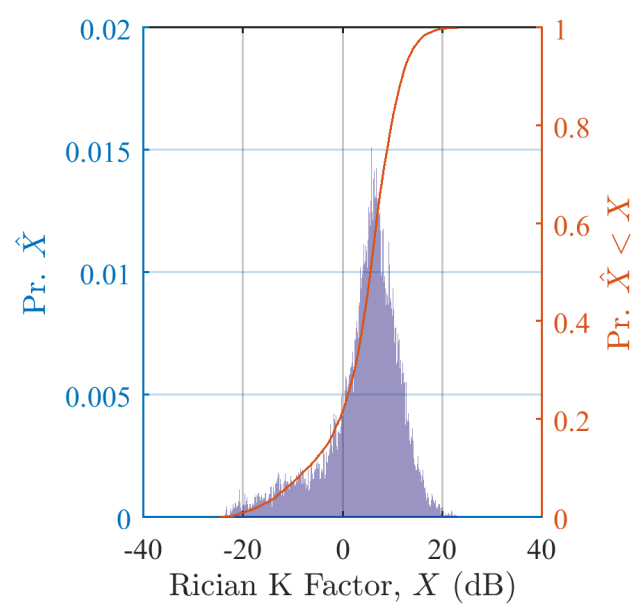

(e) Rician K-factor

Figure A.4.31: Channel characterizations for GBurgD3 2GHz TX2low crossPol run2 pp channel stats 


\section{A.4.32 GBurgD3 2GHz TX2low longPol run3 pp.mat}

Table A.96: Measurement Parameters

\begin{tabular}{l|c}
\hline Parameter & Value \\
\hline \hline Mat File & GBurgD3_2GHz_TX2low_longPol_run3_pp.mat \\
Frequency $(\mathrm{GHz})$ & 2.245 \\
Location & Machine Shop Day3 at Gaithersburg \\
RX Antenna & Omni-directional, Long Pol \\
RX Antenna Gain & -4.200 \\
TX Antenna & Omni-directional, V Pol \\
TX Antenna Gain & 2.900 \\
TX Power, Watts & 1.500 \\
PN Oversample Factor & 4.000 \\
Sample rate, MHz & 80.000
\end{tabular}

Table A.97: Statistics of Channel Estimates. Outliers are removed using a significance test of 0.95.

\begin{tabular}{l||c|c|c|c||c|c||l}
\hline Estimate & $\min (\cdot)$ & $\operatorname{median}(\cdot)$ & $\operatorname{mean}(\cdot)$ & $\max (\cdot)$ & $\operatorname{std}(\cdot)$ & $\operatorname{mad}(\cdot)$ & outlier info \\
\hline \hline$K(\mathrm{~dB})$, NLOS & -25.1 & 5.7 & 4.1 & 22.4 & 7.8 & 6.0 & N: 13203 \\
\hline$K(\mathrm{~dB})$, LOS & 4.0 & 9.9 & 10.3 & 22.4 & 2.9 & 2.4 & N: 5784 \\
\hline$\tau(\mathrm{ns})$ & 29.6 & 195.9 & 190.0 & 384.1 & 58.4 & 46.8 & N: 13203 \\
\hline$S(\mathrm{~ns})$ & 15.3 & 79.7 & 80.4 & 160.4 & 22.2 & 17.8 & N: 13203 \\
\hline
\end{tabular}

Table A.98: Statistics of Channel Estimates. Outliers are not removed. N indicates the total number of samples in the population. Outliers are provided at 10 and 20 times $\sigma$.

\begin{tabular}{l||c|c|c|c||c|c||l}
\hline Estimate & $\min (\cdot)$ & $\operatorname{median}(\cdot)$ & $\operatorname{mean}(\cdot)$ & $\max (\cdot)$ & $\operatorname{std}(\cdot)$ & $\operatorname{mad}(\cdot)$ & outlier info \\
\hline \hline$K(\mathrm{~dB})$, NLOS & -25.1 & 5.7 & 4.1 & 22.4 & 7.8 & 6.0 & N: 13203 \\
\hline$K(\mathrm{~dB})$, LOS & 4.0 & 9.9 & 10.3 & 22.4 & 2.9 & 2.4 & N: 5784 \\
\hline$\tau(\mathrm{ns})$ & 29.6 & 195.9 & 190.0 & 384.1 & 58.4 & 46.8 & N: 13203 \\
\hline$S(\mathrm{~ns})$ & 15.3 & 79.7 & 80.4 & 160.4 & 22.2 & 17.8 & N: 13203 \\
\hline
\end{tabular}




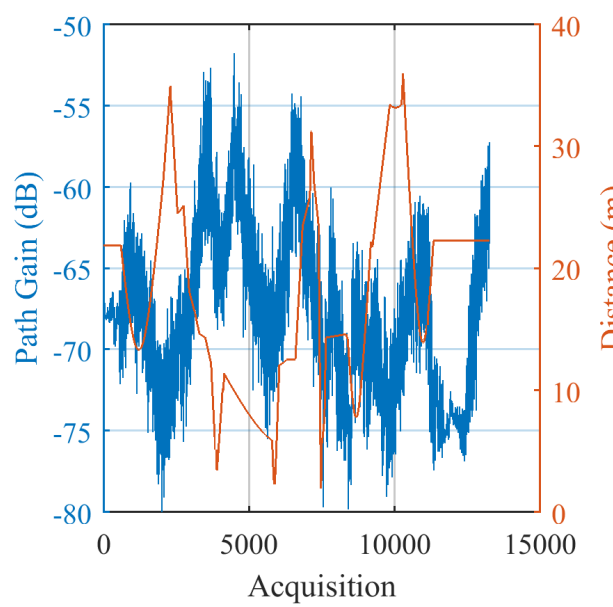

(a) Path Gain versus Acquisition Order

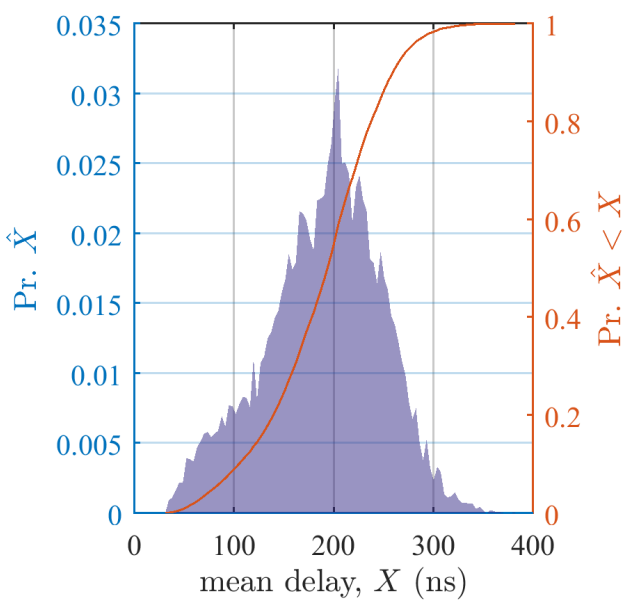

(c) Power Delay

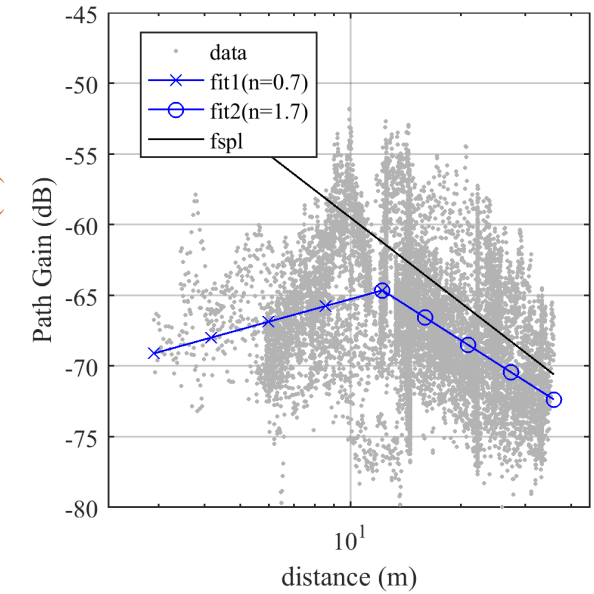

(b) Path Loss versus Distance

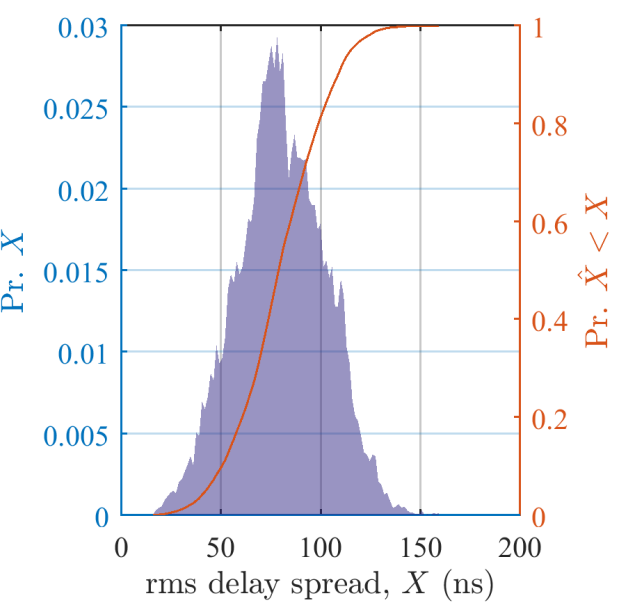

(d) Power Delay Spread

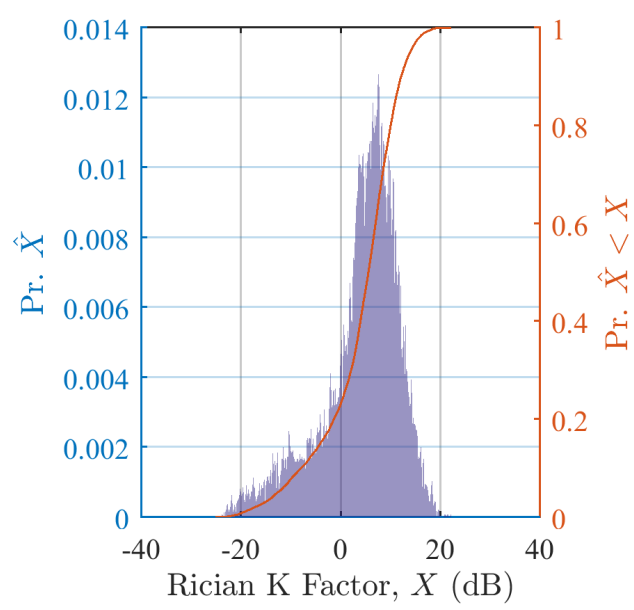

(e) Rician K-factor

Figure A.4.32: Channel characterizations for GBurgD3 2GHz TX2low longPol run3 pp channel stats 


\section{A.4.33 GBurgD3 2GHz TX2low vpol run1 pp.mat}

Table A.99: Measurement Parameters

\begin{tabular}{l|c}
\hline Parameter & Value \\
\hline \hline Mat File & GBurgD3_2GHz_TX2low_vpol_run1_pp.mat \\
Frequency (GHz) & 2.245 \\
Location & Machine Shop Day3 at Gaithersburg \\
RX Antenna & Omni-directional, V Pol \\
RX Antenna Gain & -4.200 \\
TX Antenna & Omni-directional, V Pol \\
TX Antenna Gain & 2.900 \\
TX Power, Watts & 1.500 \\
PN Oversample Factor & 4.000 \\
Sample rate, MHz & 80.000
\end{tabular}

Table A.100: Statistics of Channel Estimates. Outliers are removed using a significance test of 0.95.

\begin{tabular}{l||c|c|c|c||c|c||l}
\hline Estimate & $\min (\cdot)$ & $\operatorname{median}(\cdot)$ & $\operatorname{mean}(\cdot)$ & $\max (\cdot)$ & $\operatorname{std}(\cdot)$ & $\operatorname{mad}(\cdot)$ & outlier info \\
\hline \hline$K(\mathrm{~dB})$, NLOS & -25.5 & 8.8 & 7.5 & 27.7 & 7.7 & 5.9 & N: 9979 \\
\hline$K(\mathrm{~dB})$, LOS & 3.7 & 11.7 & 12.2 & 27.7 & 3.8 & 3.1 & N: 6179 \\
\hline$\tau(\mathrm{ns})$ & 35.4 & 160.0 & 160.6 & 364.4 & 65.2 & 55.4 & N: 9979 \\
\hline$S(\mathrm{~ns})$ & 13.5 & 65.1 & 64.9 & 146.9 & 25.0 & 20.6 & N: 9979 \\
\hline
\end{tabular}

Table A.101: Statistics of Channel Estimates. Outliers are not removed. N indicates the total number of samples in the population. Outliers are provided at 10 and 20 times $\sigma$.

\begin{tabular}{l||c|c|c|c||c|c||l}
\hline Estimate & $\min (\cdot)$ & $\operatorname{median}(\cdot)$ & $\operatorname{mean}(\cdot)$ & $\max (\cdot)$ & $\operatorname{std}(\cdot)$ & $\operatorname{mad}(\cdot)$ & outlier info \\
\hline \hline$K(\mathrm{~dB})$, NLOS & -25.5 & 8.8 & 7.5 & 27.7 & 7.7 & 5.9 & N: 9979 \\
\hline$K(\mathrm{~dB})$, LOS & 3.7 & 11.7 & 12.2 & 27.7 & 3.8 & 3.1 & N: 6179 \\
\hline$\tau(\mathrm{ns})$ & 35.4 & 160.0 & 160.6 & 364.4 & 65.2 & 55.4 & N: 9979 \\
\hline$S(\mathrm{~ns})$ & 13.5 & 65.1 & 64.9 & 146.9 & 25.0 & 20.6 & N: 9979 \\
\hline
\end{tabular}




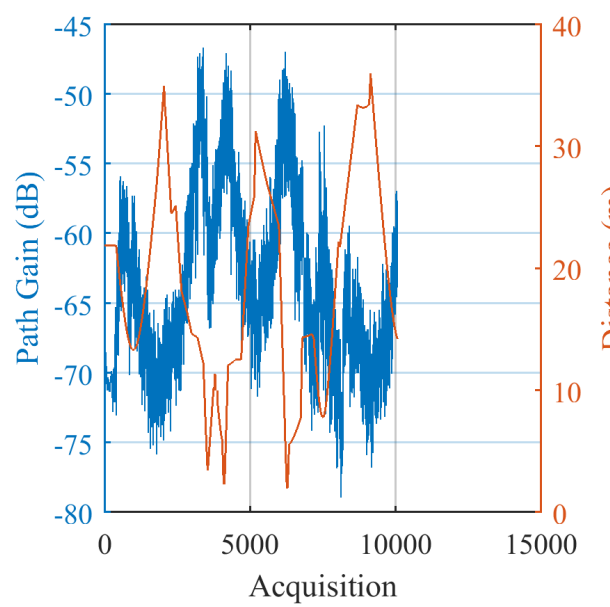

(a) Path Gain versus Acquisition Order

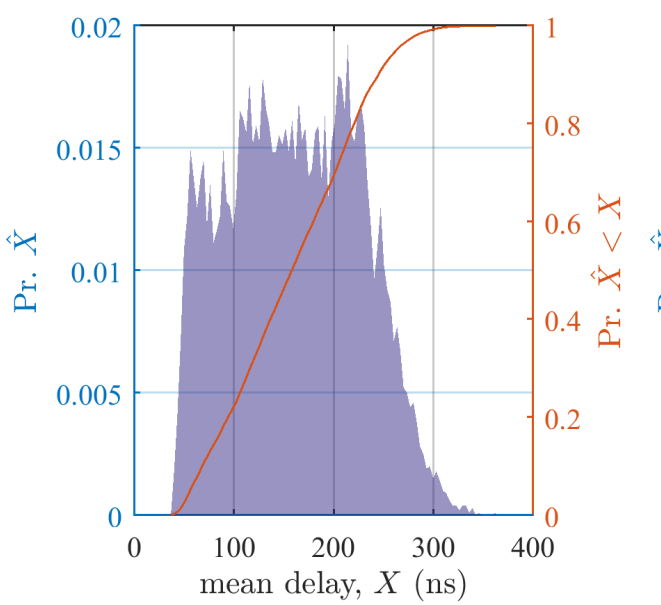

(c) Power Delay

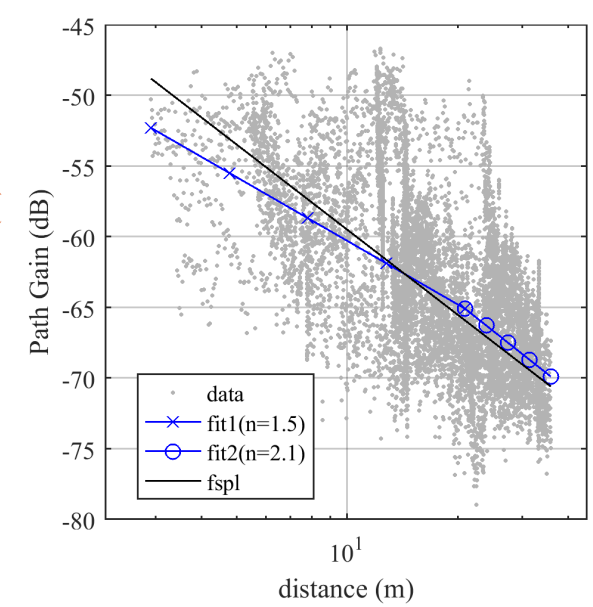

(b) Path Loss versus Distance

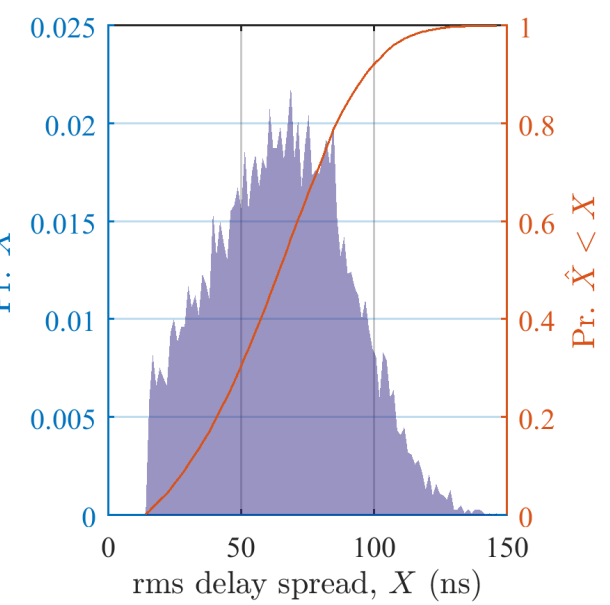

(d) Power Delay Spread

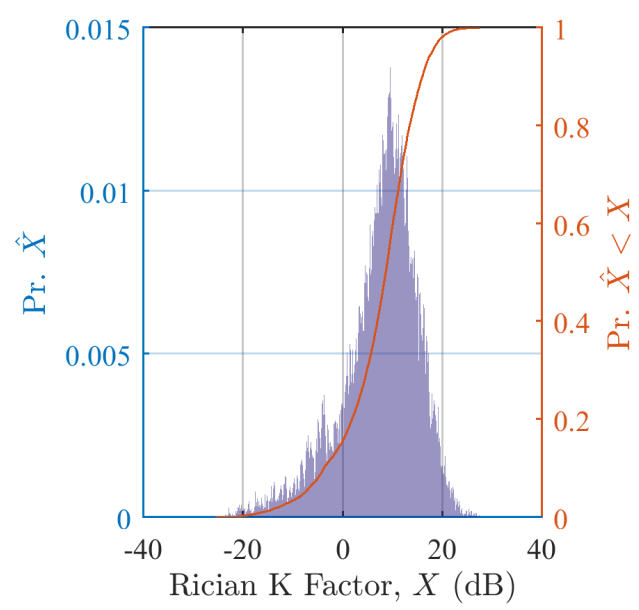

(e) Rician K-factor

Figure A.4.33: Channel characterizations for GBurgD3 2GHz TX2low vpol run1 pp channel stats 


\section{A.4.34 GburgD2 5GHz TX2high vpol pp.mat}

Table A.102: Measurement Parameters

\begin{tabular}{l|c}
\hline Parameter & Value \\
\hline \hline Mat File & GburgD2_5GHz_TX2high_vpol_pp.mat \\
Frequency (GHz) & 5.400 \\
Location & Machine Shop Day2 at Gaithersburg \\
RX Antenna & Omni-directional, V Pol \\
RX Antenna Gain & -3.500 \\
TX Antenna & Omni-directional, V Pol \\
TX Antenna Gain & 3.600 \\
TX Power, Watts & 1.250 \\
PN Oversample Factor & 4.000 \\
Sample rate, MHz & 80.000
\end{tabular}

Table A.103: Statistics of Channel Estimates. Outliers are removed using a significance test of 0.95.

\begin{tabular}{l||c|c|c|c||c|c||l}
\hline Estimate & $\min (\cdot)$ & $\operatorname{median}(\cdot)$ & $\operatorname{mean}(\cdot)$ & $\max (\cdot)$ & $\operatorname{std}(\cdot)$ & $\operatorname{mad}(\cdot)$ & outlier info \\
\hline \hline$K(\mathrm{~dB})$, NLOS & -23.3 & 8.4 & 7.5 & 25.6 & 7.4 & 5.2 & N: 10491 \\
\hline$K(\mathrm{~dB})$, LOS & 3.3 & 11.1 & 11.4 & 25.6 & 3.5 & 2.9 & N: 6827 \\
\hline$\tau(\mathrm{ns})$ & 19.4 & 137.7 & 135.1 & 298.8 & 50.0 & 42.4 & N: 10491 \\
\hline$S(\mathrm{~ns})$ & 13.6 & 66.2 & 66.8 & 137.5 & 22.3 & 18.5 & N: 10491 \\
\hline
\end{tabular}

Table A.104: Statistics of Channel Estimates. Outliers are not removed. N indicates the total number of samples in the population. Outliers are provided at 10 and 20 times $\sigma$.

\begin{tabular}{l||c|c|c|c||c|c||l}
\hline Estimate & $\min (\cdot)$ & $\operatorname{median}(\cdot)$ & $\operatorname{mean}(\cdot)$ & $\max (\cdot)$ & $\operatorname{std}(\cdot)$ & $\operatorname{mad}(\cdot)$ & outlier info \\
\hline \hline$K(\mathrm{~dB})$, NLOS & -23.3 & 8.4 & 7.5 & 25.6 & 7.4 & 5.2 & N: 10491 \\
\hline$K(\mathrm{~dB})$, LOS & 3.3 & 11.1 & 11.4 & 25.6 & 3.5 & 2.9 & N: 6827 \\
\hline$\tau(\mathrm{ns})$ & 19.4 & 137.7 & 135.1 & 298.8 & 50.0 & 42.4 & N: 10491 \\
\hline$S(\mathrm{~ns})$ & 13.6 & 66.2 & 66.8 & 137.5 & 22.3 & 18.5 & N: 10491 \\
\hline
\end{tabular}




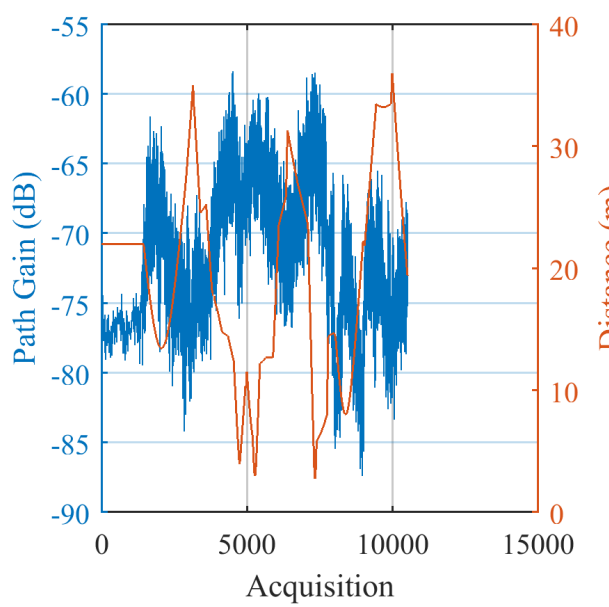

(a) Path Gain versus Acquisition Order

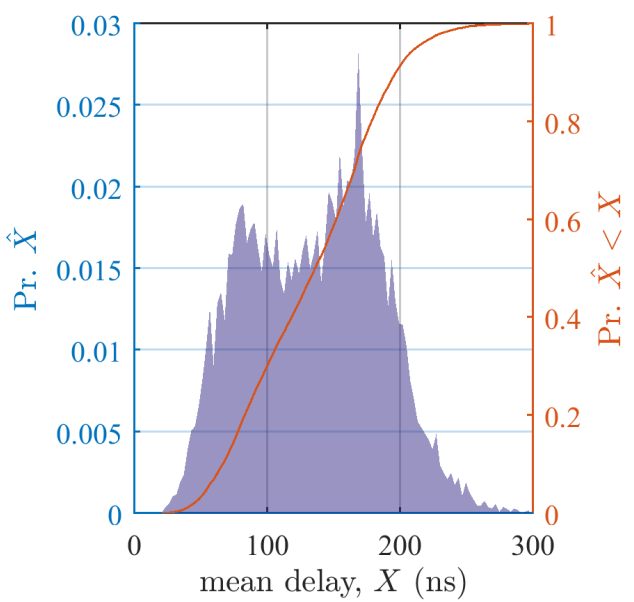

(c) Power Delay

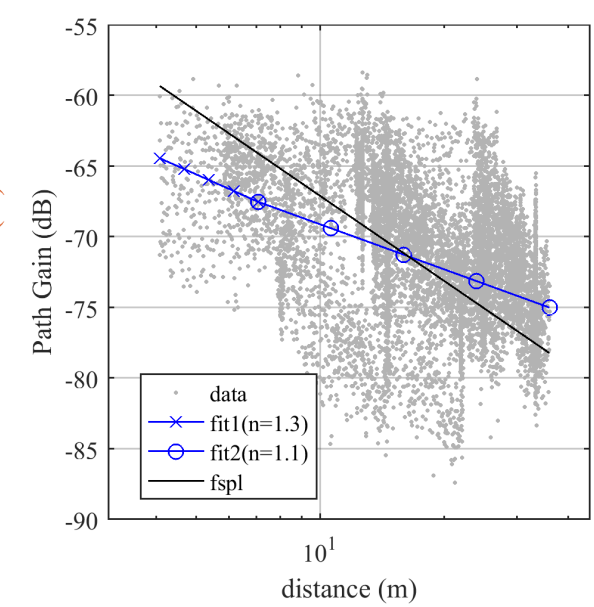

(b) Path Loss versus Distance

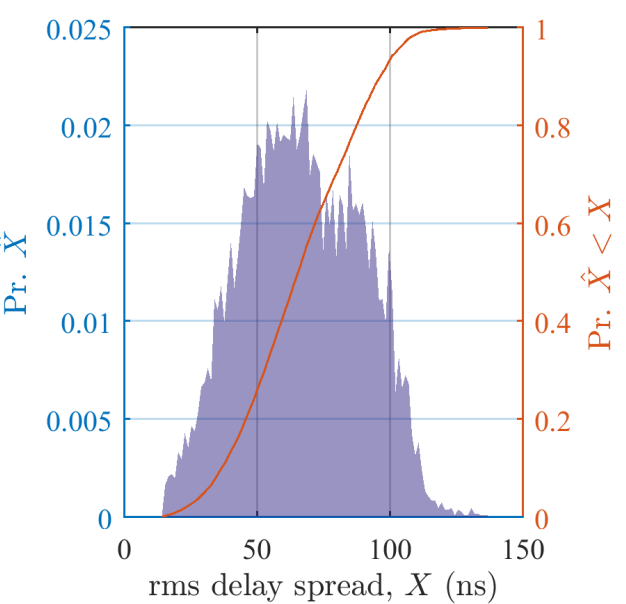

(d) Power Delay Spread

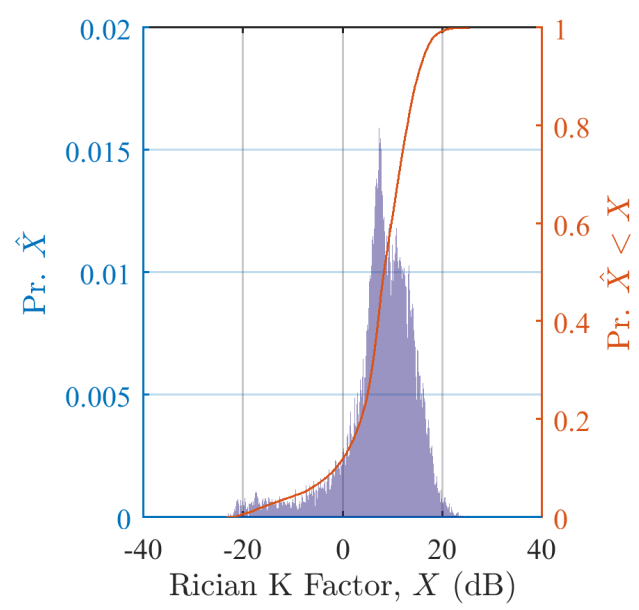

(e) Rician K-factor

Figure A.4.34: Channel characterizations for GburgD2 5GHz TX2high vpol pp channel stats 


\section{A.4.35 GburgD3 2GHz TX2high vpol run1 pp.mat}

Table A.105: Measurement Parameters

\begin{tabular}{l|c}
\hline Parameter & Value \\
\hline \hline Mat File & GburgD3_2GHz_TX2high_vpol_run1_pp.mat \\
Frequency (GHz) & 2.245 \\
Location & Machine Shop Day3 at Gaithersburg \\
RX Antenna & Omni-directional, V Pol \\
RX Antenna Gain & -4.200 \\
TX Antenna & Omni-directional, V Pol \\
TX Antenna Gain & 2.900 \\
TX Power, Watts & 1.500 \\
PN Oversample Factor & 4.000 \\
Sample rate, MHz & 80.000
\end{tabular}

Table A.106: Statistics of Channel Estimates. Outliers are removed using a significance test of 0.95.

\begin{tabular}{l||c|c|c|c||c|c||l}
\hline Estimate & $\min (\cdot)$ & $\operatorname{median}(\cdot)$ & $\operatorname{mean}(\cdot)$ & $\max (\cdot)$ & $\operatorname{std}(\cdot)$ & $\operatorname{mad}(\cdot)$ & outlier info \\
\hline \hline$K(\mathrm{~dB})$, NLOS & -19.9 & 9.0 & 8.7 & 26.5 & 6.3 & 4.6 & N: 9487 \\
\hline$K(\mathrm{~dB})$, LOS & 4.0 & 11.3 & 11.8 & 26.5 & 3.6 & 3.0 & N: 6570 \\
\hline$\tau(\mathrm{ns})$ & 42.6 & 160.0 & 155.2 & 340.5 & 57.6 & 48.5 & N: 9499 \\
\hline$S(\mathrm{~ns})$ & 13.5 & 66.2 & 65.7 & 155.9 & 24.1 & 19.8 & N: 9499 \\
\hline
\end{tabular}

Table A.107: Statistics of Channel Estimates. Outliers are not removed. N indicates the total number of samples in the population. Outliers are provided at 10 and 20 times $\sigma$.

\begin{tabular}{l||c|c|c|c||c|c||l}
\hline Estimate & $\min (\cdot)$ & $\operatorname{median}(\cdot)$ & $\operatorname{mean}(\cdot)$ & $\max (\cdot)$ & $\operatorname{std}(\cdot)$ & $\operatorname{mad}(\cdot)$ & outlier info \\
\hline \hline$K(\mathrm{~dB})$, NLOS & -22.8 & 9.0 & 8.7 & 26.5 & 6.4 & 4.7 & N: 9499 \\
\hline$K(\mathrm{~dB})$, LOS & 4.0 & 11.3 & 11.8 & 26.5 & 3.6 & 3.0 & N: 6570 \\
\hline$\tau(\mathrm{ns})$ & 42.6 & 160.0 & 155.2 & 340.5 & 57.6 & 48.5 & N: 9499 \\
\hline$S(\mathrm{~ns})$ & 13.5 & 66.2 & 65.7 & 155.9 & 24.1 & 19.8 & N: 9499 \\
\hline
\end{tabular}




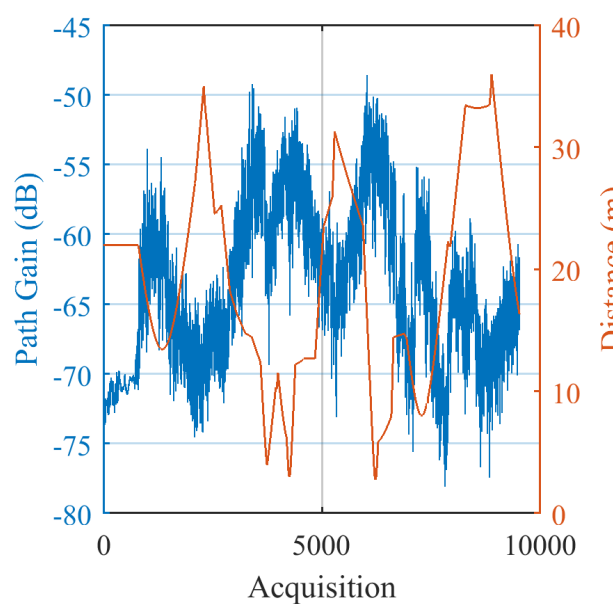

(a) Path Gain versus Acquisition Order

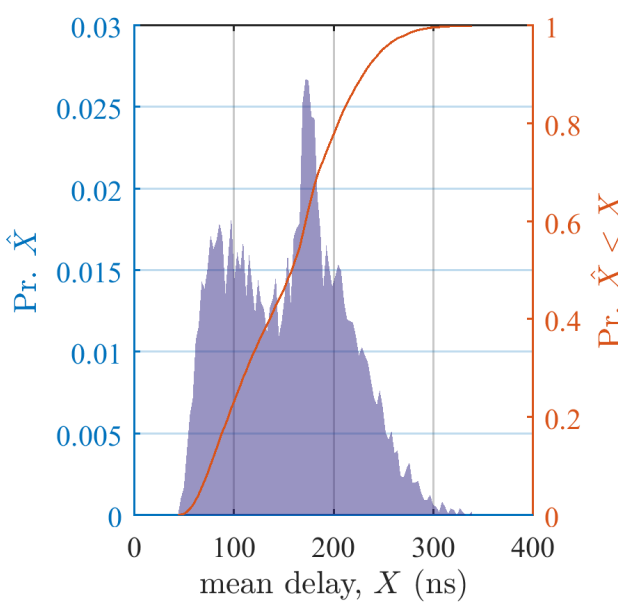

(c) Power Delay

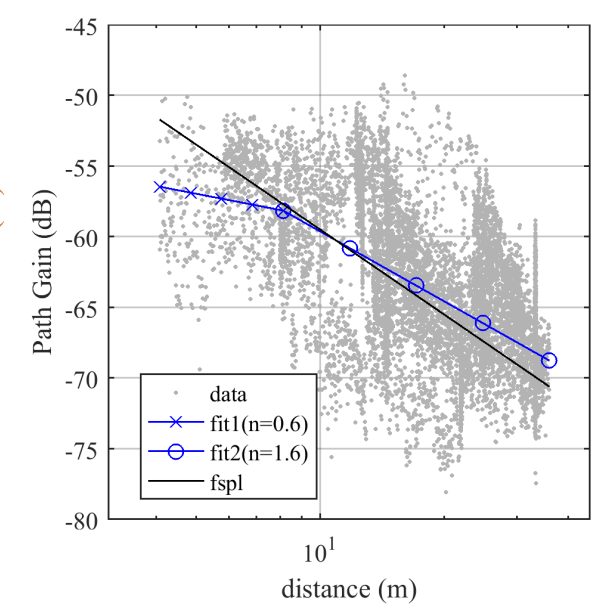

(b) Path Loss versus Distance

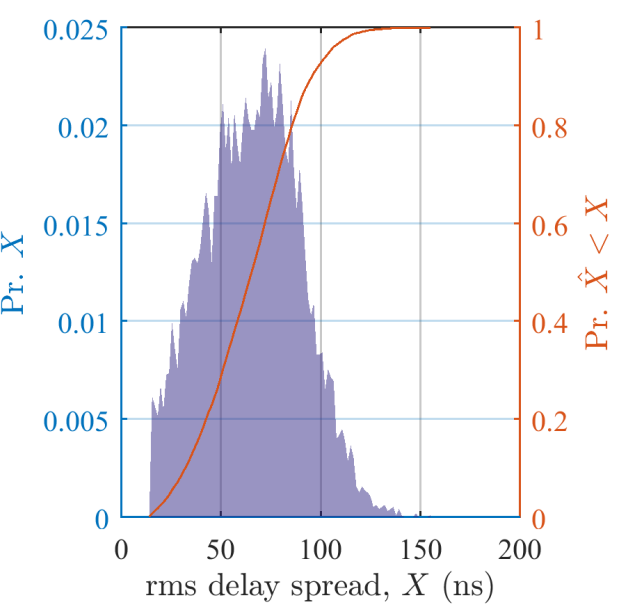

(d) Power Delay Spread

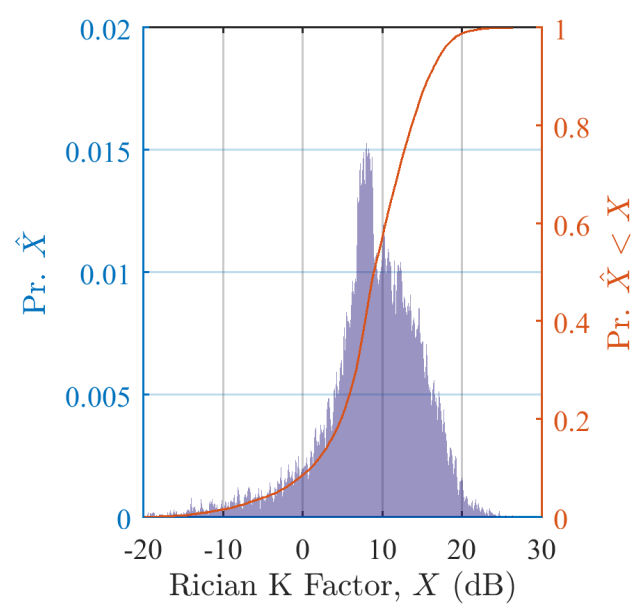

(e) Rician K-factor

Figure A.4.35: Channel characterizations for GburgD3 2GHz TX2high vpol run1 pp channel stats 


\section{A.4.36 Oats 2G vpol 3115 run40 pp.mat}

Table A.108: Measurement Parameters

\begin{tabular}{l|c}
\hline Parameter & Value \\
\hline \hline Mat File & Oats_2G_vpol_3115_run40_pp.mat \\
Frequency (GHz) & 2.245 \\
Location & Boulder OATS Day 5 $(2016-02-17)$ at Boulder OATS \\
RX Antenna & Omni-directional, V Pol \\
RX Antenna Gain & -4.200 \\
TX Antenna & Horn Antenna, V Pol \\
TX Antenna Gain & 9.260 \\
TX Power, Watts & 6.550 \\
PN Oversample Factor & 4.000 \\
Sample rate, MHz & 200.000
\end{tabular}

Table A.109: Statistics of Channel Estimates. Outliers are removed using a significance test of 0.95.

\begin{tabular}{l||c|c|c|c||c|c||l}
\hline Estimate & $\min (\cdot)$ & $\operatorname{median}(\cdot)$ & $\operatorname{mean}(\cdot)$ & $\max (\cdot)$ & $\operatorname{std}(\cdot)$ & $\operatorname{mad}(\cdot)$ & outlier info \\
\hline \hline$K(\mathrm{~dB})$, NLOS & 1.4 & 25.7 & 24.0 & 30.0 & 5.6 & 4.3 & N: 2999 \\
\hline$K(\mathrm{~dB})$, LOS & 1.4 & 25.7 & 24.0 & 30.0 & 5.6 & 4.3 & N: 2999 \\
\hline$\tau(\mathrm{ns})$ & 0.0 & 36.5 & 43.9 & 117.2 & 27.8 & 22.5 & N: 13160 \\
\hline$S(\mathrm{~ns})$ & 5.3 & 6.2 & 6.2 & 7.4 & 0.3 & 0.2 & N: 12104 \\
\hline
\end{tabular}

Table A.110: Statistics of Channel Estimates. Outliers are not removed. N indicates the total number of samples in the population. Outliers are provided at 10 and 20 times $\sigma$.

\begin{tabular}{l||c|c|c|c||c|c||l}
\hline Estimate & $\min (\cdot)$ & $\operatorname{median}(\cdot)$ & $\operatorname{mean}(\cdot)$ & $\max (\cdot)$ & $\operatorname{std}(\cdot)$ & $\operatorname{mad}(\cdot)$ & outlier info \\
\hline \hline$K(\mathrm{~dB})$, NLOS & -0.7 & 25.7 & 24.0 & 30.0 & 5.6 & 4.3 & N: 3000 \\
\hline$K(\mathrm{~dB})$, LOS & 1.4 & 25.7 & 24.0 & 30.0 & 5.6 & 4.3 & N: 2999 \\
\hline$\tau(\mathrm{ns})$ & 0.0 & 36.5 & 43.9 & 117.2 & 27.8 & 22.5 & N: 13160 \\
\hline$S(\mathrm{~ns})$ & 0.0 & 6.2 & 6.0 & 8.2 & 1.1 & 0.5 & N: 13160 \\
\hline
\end{tabular}




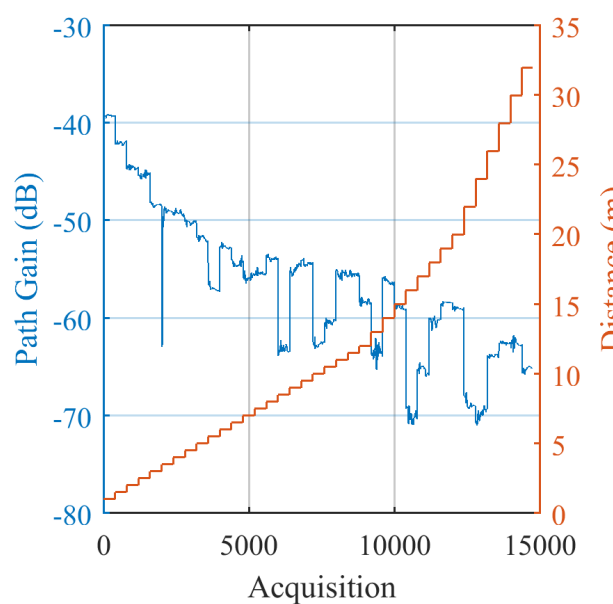

(a) Path Gain versus Acquisition Order

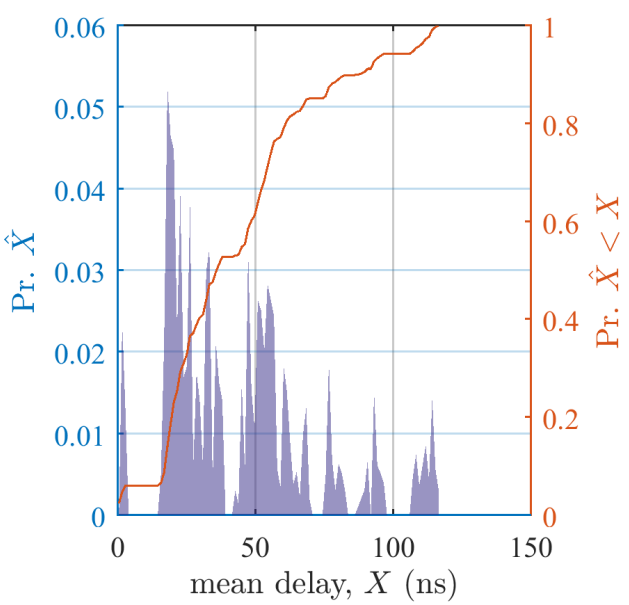

(c) Power Delay

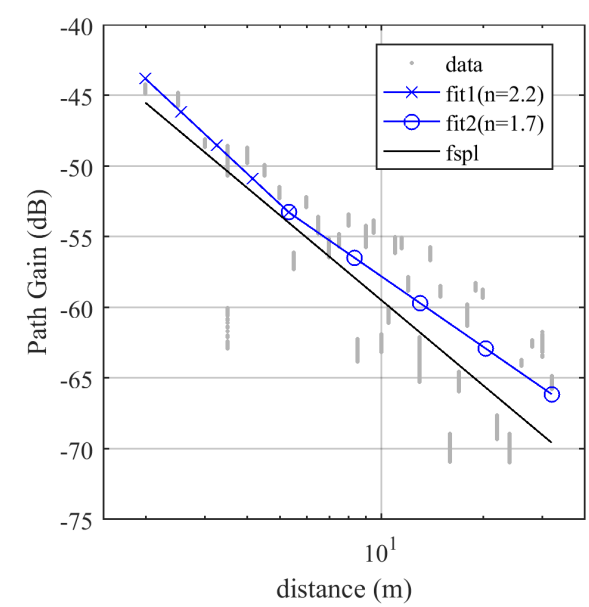

(b) Path Loss versus Distance

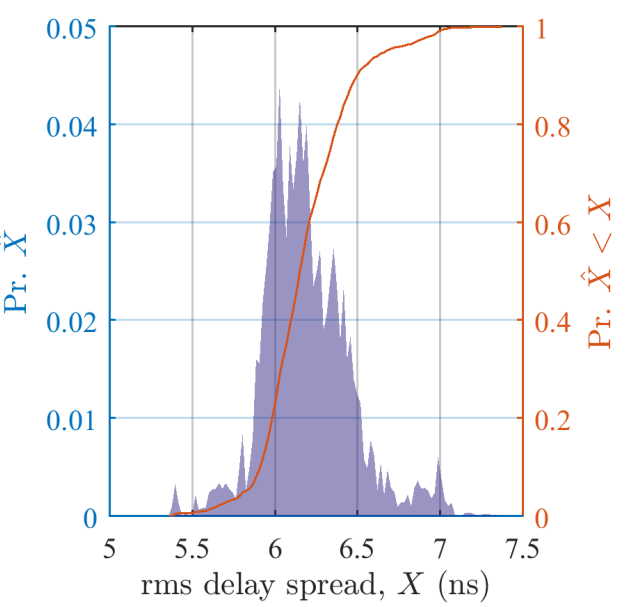

(d) Power Delay Spread

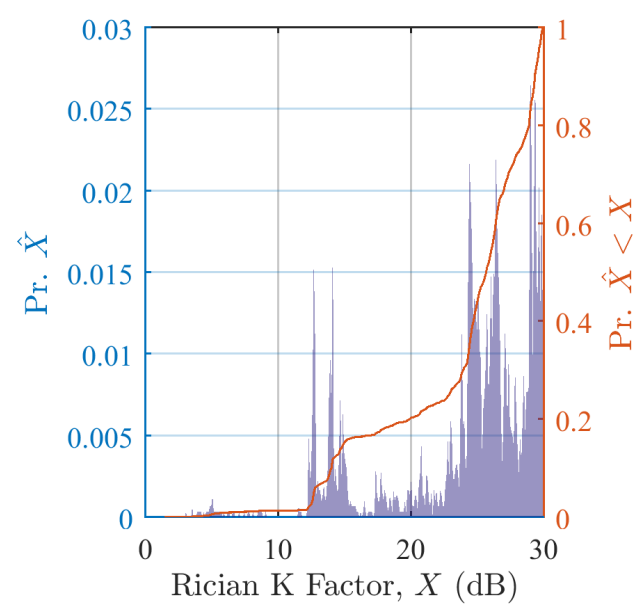

(e) Rician K-factor

Figure A.4.36: Channel characterizations for Oats $2 \mathrm{G}$ vpol 3115 run40 pp channel stats 


\section{A.4.37 Oats 2G vpol run39 pp.mat}

Table A.111: Measurement Parameters

\begin{tabular}{l|c}
\hline Parameter & Value \\
\hline \hline Mat File & Oats_2G_vpol_run39_pp.mat \\
Frequency $(\mathrm{GHz})$ & 2.245 \\
Location & Boulder OATS Day 5 $(2016-02-17)$ at Boulder OATS \\
RX Antenna & Omni-directional, V Pol \\
RX Antenna Gain & -4.200 \\
TX Antenna & Omni-directional, V Pol \\
TX Antenna Gain & 2.900 \\
TX Power, Watts & 6.550 \\
PN Oversample Factor & 4.000 \\
Sample rate, MHz & 200.000
\end{tabular}

Table A.112: Statistics of Channel Estimates. Outliers are removed using a significance test of 0.95.

\begin{tabular}{l||c|c|c|c||c|c||l}
\hline Estimate & $\min (\cdot)$ & $\operatorname{median}(\cdot)$ & $\operatorname{mean}(\cdot)$ & $\max (\cdot)$ & $\operatorname{std}(\cdot)$ & $\operatorname{mad}(\cdot)$ & outlier info \\
\hline \hline$K(\mathrm{~dB})$, NLOS & -21.1 & 25.0 & 19.7 & 30.0 & 11.7 & 9.0 & N: 7740 \\
\hline$K(\mathrm{~dB})$, LOS & 0.0 & 25.5 & 22.4 & 30.0 & 7.4 & 6.2 & N: 7079 \\
\hline$\tau(\mathrm{ns})$ & 1.1 & 9.5 & 17.4 & 76.6 & 18.6 & 14.8 & N: 12800 \\
\hline$S(\mathrm{~ns})$ & 2.1 & 5.6 & 5.5 & 13.9 & 1.8 & 1.3 & N: 12032 \\
\hline
\end{tabular}

Table A.113: Statistics of Channel Estimates. Outliers are not removed. N indicates the total number of samples in the population. Outliers are provided at 10 and 20 times $\sigma$.

\begin{tabular}{l||c|c|c|c||c|c||l}
\hline Estimate & $\min (\cdot)$ & $\operatorname{median}(\cdot)$ & $\operatorname{mean}(\cdot)$ & $\max (\cdot)$ & $\operatorname{std}(\cdot)$ & $\operatorname{mad}(\cdot)$ & outlier info \\
\hline \hline$K(\mathrm{~dB})$, NLOS & -21.1 & 25.0 & 19.7 & 30.0 & 11.7 & 9.0 & N: 7740 \\
\hline$K(\mathrm{~dB})$, LOS & 0.0 & 25.5 & 22.4 & 30.0 & 7.4 & 6.2 & N: 7079 \\
\hline$\tau(\mathrm{ns})$ & 1.1 & 9.5 & 17.4 & 76.6 & 18.6 & 14.8 & N: 12800 \\
\hline$S(\mathrm{~ns})$ & 2.1 & 5.9 & 6.4 & 44.4 & 4.3 & 2.4 & N: 12800 \\
\hline
\end{tabular}




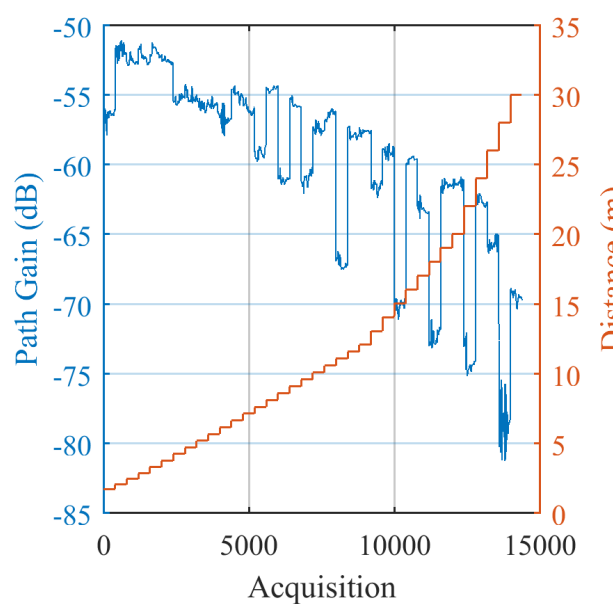

(a) Path Gain versus Acquisition Order

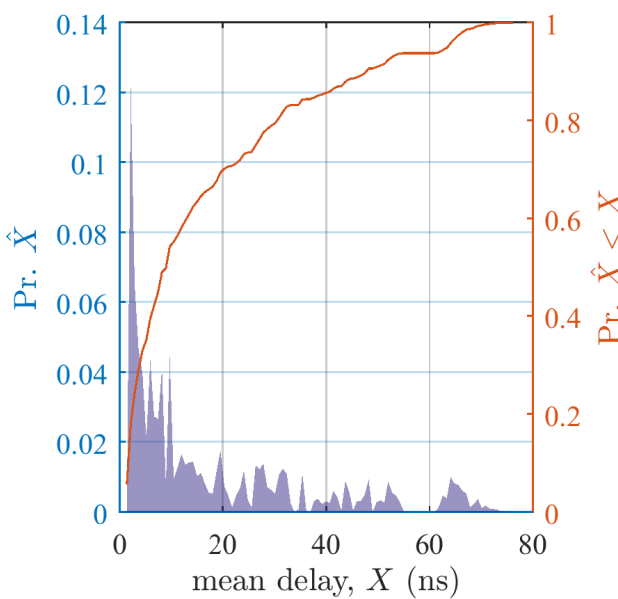

(c) Power Delay

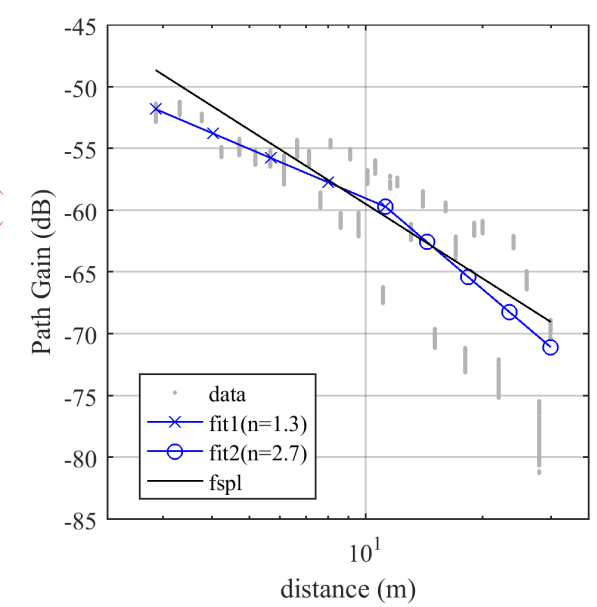

(b) Path Loss versus Distance

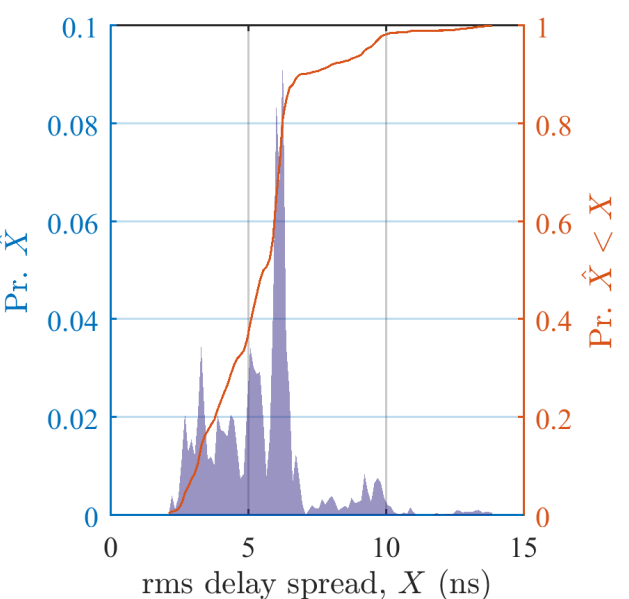

(d) Power Delay Spread

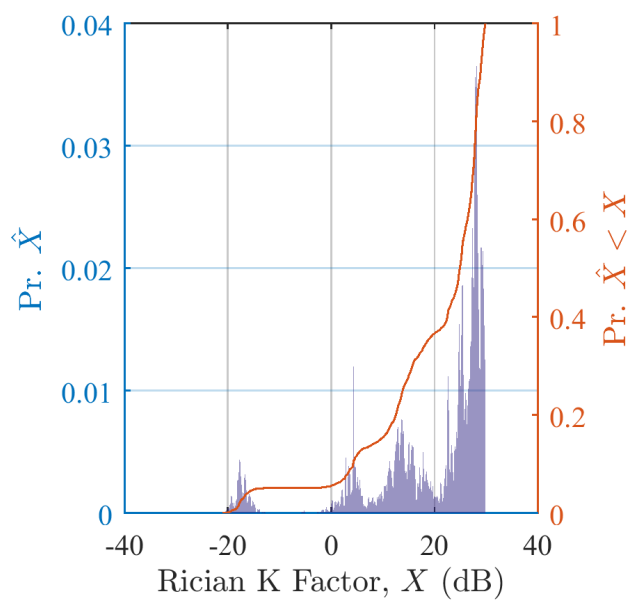

(e) Rician K-factor

Figure A.4.37: Channel characterizations for Oats $2 \mathrm{G}$ vpol run39 pp channel stats 


\section{A.4.38 Oats 5G 3115horn vpol run36b pp.mat}

Table A.114: Measurement Parameters

\begin{tabular}{l|c}
\hline Parameter & Value \\
\hline \hline Mat File & Oats_5G_3115horn_vpol_run36b_pp.mat \\
Frequency (GHz) & 5.400 \\
Location & Boulder OATS Day 5 $(2016-02-17)$ at Boulder OATS \\
RX Antenna & Omni-directional, V Pol \\
RX Antenna Gain & -3.500 \\
TX Antenna & Horn Antenna, V Pol \\
TX Antenna Gain & 10.320 \\
TX Power, Watts & 3.640 \\
PN Oversample Factor & 4.000 \\
Sample rate, MHz & 200.000
\end{tabular}

Table A.115: Statistics of Channel Estimates. Outliers are removed using a significance test of 0.95.

\begin{tabular}{l||c|c|c|c||c|c||l}
\hline Estimate & $\min (\cdot)$ & $\operatorname{median}(\cdot)$ & $\operatorname{mean}(\cdot)$ & $\max (\cdot)$ & $\operatorname{std}(\cdot)$ & $\operatorname{mad}(\cdot)$ & outlier info \\
\hline \hline$K(\mathrm{~dB})$, NLOS & -17.3 & 17.3 & 12.2 & 30.0 & 14.8 & 12.1 & N: 1776 \\
\hline$K(\mathrm{~dB})$, LOS & 3.1 & 19.3 & 19.7 & 30.0 & 6.2 & 5.1 & N: 1376 \\
\hline$\tau(\mathrm{ns})$ & 4.0 & 16.2 & 24.4 & 79.5 & 18.2 & 15.4 & N: 14200 \\
\hline$S(\mathrm{~ns})$ & 3.9 & 6.2 & 6.1 & 8.7 & 0.5 & 0.4 & N: 14163 \\
\hline
\end{tabular}

Table A.116: Statistics of Channel Estimates. Outliers are not removed. N indicates the total number of samples in the population. Outliers are provided at 10 and 20 times $\sigma$.

\begin{tabular}{l||c|c|c|c||c|c||l}
\hline Estimate & $\min (\cdot)$ & $\operatorname{median}(\cdot)$ & $\operatorname{mean}(\cdot)$ & $\max (\cdot)$ & $\operatorname{std}(\cdot)$ & $\operatorname{mad}(\cdot)$ & outlier info \\
\hline \hline$K(\mathrm{~dB})$, NLOS & -17.3 & 17.3 & 12.2 & 30.0 & 14.8 & 12.1 & N: 1776 \\
\hline$K(\mathrm{~dB})$, LOS & 3.1 & 19.3 & 19.7 & 30.0 & 6.2 & 5.1 & N: 1376 \\
\hline$\tau(\mathrm{ns})$ & 4.0 & 16.2 & 24.4 & 79.5 & 18.2 & 15.4 & N: 14200 \\
\hline$S(\mathrm{~ns})$ & 3.9 & 6.2 & 6.2 & 10.9 & 0.6 & 0.4 & N: 14200 \\
\hline
\end{tabular}




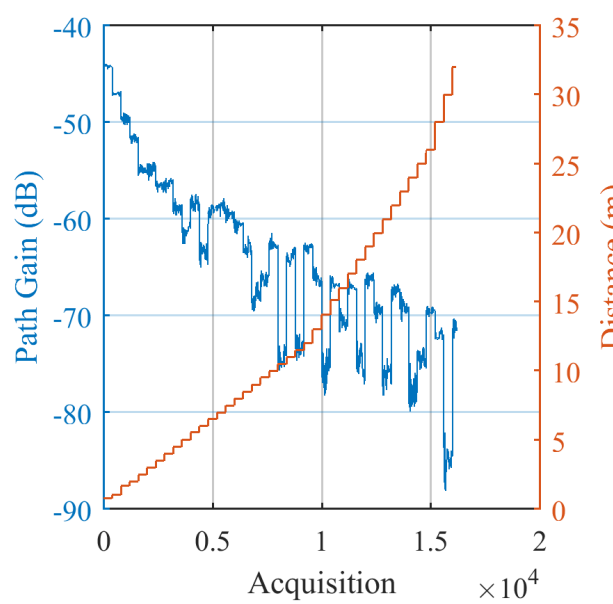

(a) Path Gain versus Acquisition Order

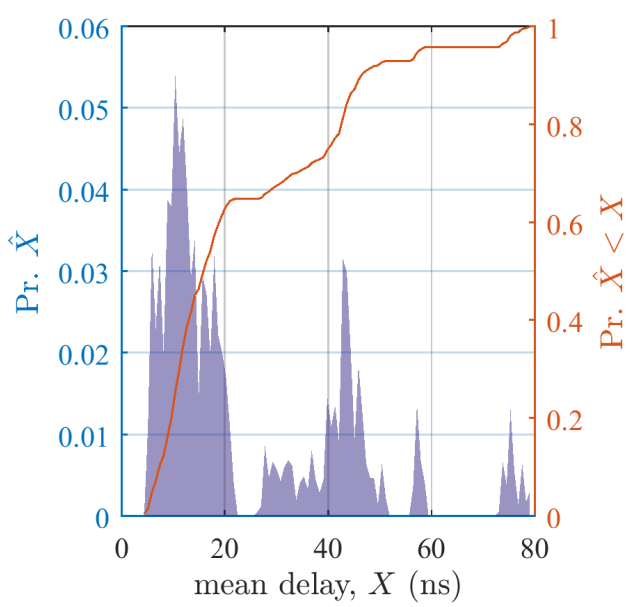

(c) Power Delay

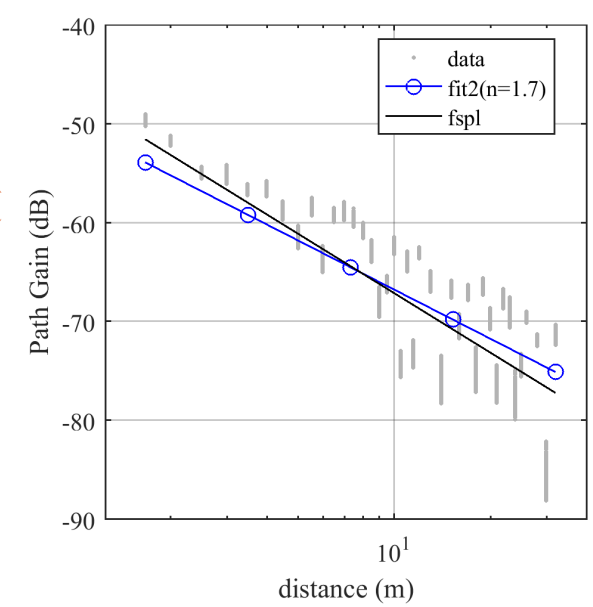

(b) Path Loss versus Distance

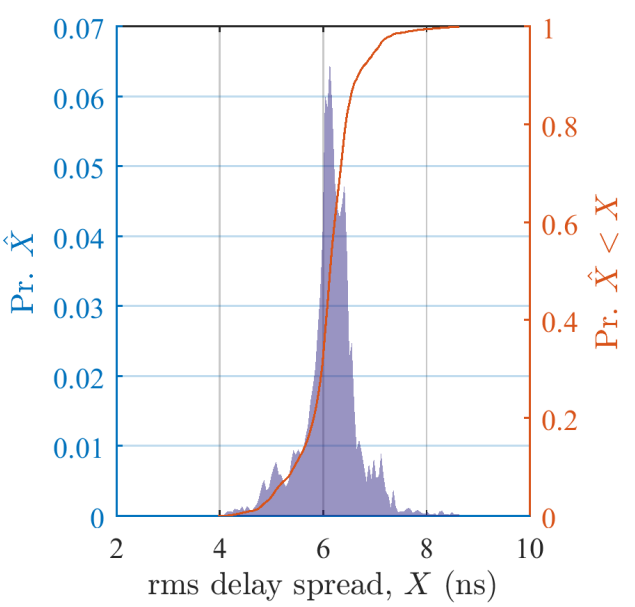

(d) Power Delay Spread

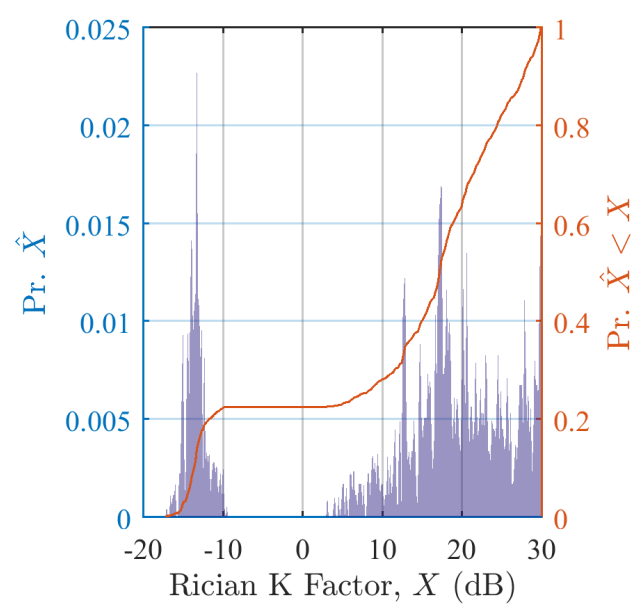

(e) Rician K-factor

Figure A.4.38: Channel characterizations for Oats 5G 3115horn vpol run36b pp channel stats 


\section{A.4.39 Tx2 2G Boiler 3115Vpol run1 pp.mat}

Table A.117: Measurement Parameters

\begin{tabular}{l|c}
\hline Parameter & Value \\
\hline \hline Mat File & Tx2 2G Boiler 3115Vpol run1 \\
Frequency $(\mathrm{GHz})$ & 2.245 \\
Location & Boulder CUP Day 2 (2016-02-10) at Boulder \\
RX Antenna & Omni-directional, V Pol \\
RX Antenna Gain & -4.200 \\
TX Antenna & Horn Antenna, V Pol \\
TX Antenna Gain & 9.260 \\
TX Power, Watts & 1.780 \\
PN Oversample Factor & 4.000 \\
Sample rate, MHz & 200.000
\end{tabular}

Table A.118: Statistics of Channel Estimates. Outliers are removed using a significance test of 0.95.

\begin{tabular}{l||c|c|c|c||c|c||l}
\hline Estimate & $\min (\cdot)$ & $\operatorname{median}(\cdot)$ & $\operatorname{mean}(\cdot)$ & $\max (\cdot)$ & $\operatorname{std}(\cdot)$ & $\operatorname{mad}(\cdot)$ & outlier info \\
\hline \hline$K(\mathrm{~dB})$, NLOS & -22.8 & 10.6 & 9.5 & 24.8 & 7.4 & 5.6 & N: 11398 \\
\hline$K(\mathrm{~dB})$, LOS & 2.8 & 13.3 & 13.3 & 24.8 & 3.8 & 3.1 & N: 7802 \\
\hline$\tau(\mathrm{ns})$ & 1.2 & 64.6 & 69.7 & 202.8 & 44.0 & 35.3 & N: 11398 \\
\hline$S(\mathrm{~ns})$ & 2.4 & 30.1 & 32.1 & 86.7 & 17.0 & 14.3 & N: 11398 \\
\hline
\end{tabular}

Table A.119: Statistics of Channel Estimates. Outliers are not removed. N indicates the total number of samples in the population. Outliers are provided at 10 and 20 times $\sigma$.

\begin{tabular}{l||c|c|c|c||c|c||l}
\hline Estimate & $\min (\cdot)$ & $\operatorname{median}(\cdot)$ & $\operatorname{mean}(\cdot)$ & $\max (\cdot)$ & $\operatorname{std}(\cdot)$ & $\operatorname{mad}(\cdot)$ & outlier info \\
\hline \hline$K(\mathrm{~dB})$, NLOS & -22.8 & 10.6 & 9.5 & 24.8 & 7.4 & 5.6 & N: 11398 \\
\hline$K(\mathrm{~dB})$, LOS & 2.8 & 13.3 & 13.3 & 24.8 & 3.8 & 3.1 & N: 7802 \\
\hline$\tau(\mathrm{ns})$ & 1.2 & 64.6 & 69.7 & 202.8 & 44.0 & 35.3 & N: 11398 \\
\hline$S(\mathrm{~ns})$ & 2.4 & 30.1 & 32.1 & 86.7 & 17.0 & 14.3 & N: 11398 \\
\hline
\end{tabular}




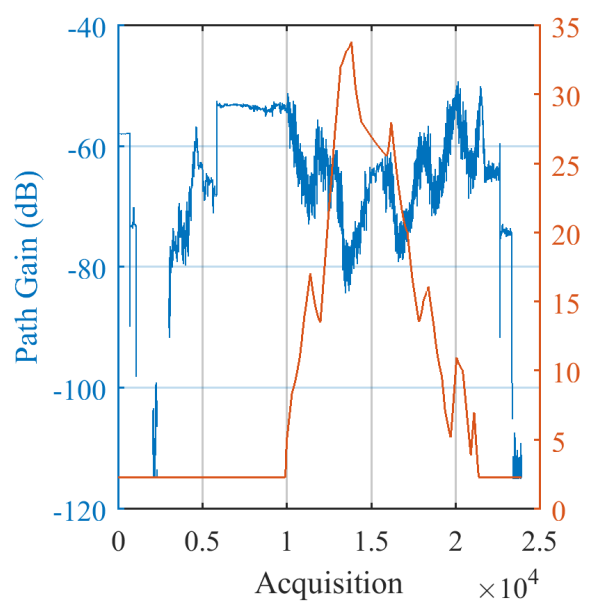

(a) Path Gain versus Acquisition Order

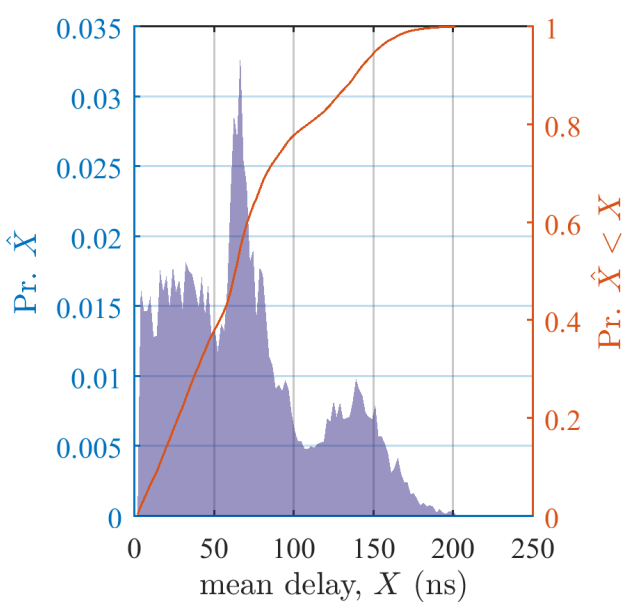

(c) Power Delay

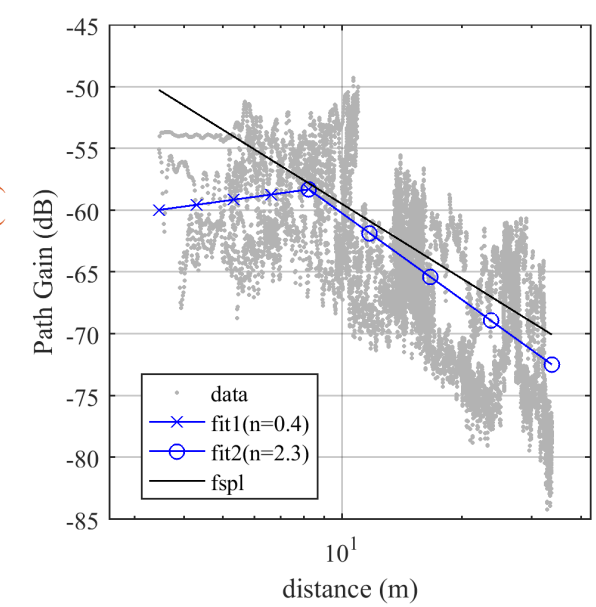

(b) Path Loss versus Distance

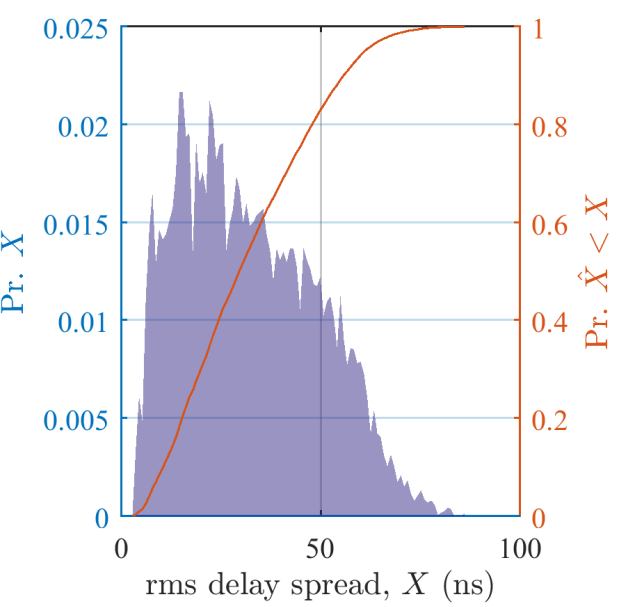

(d) Power Delay Spread

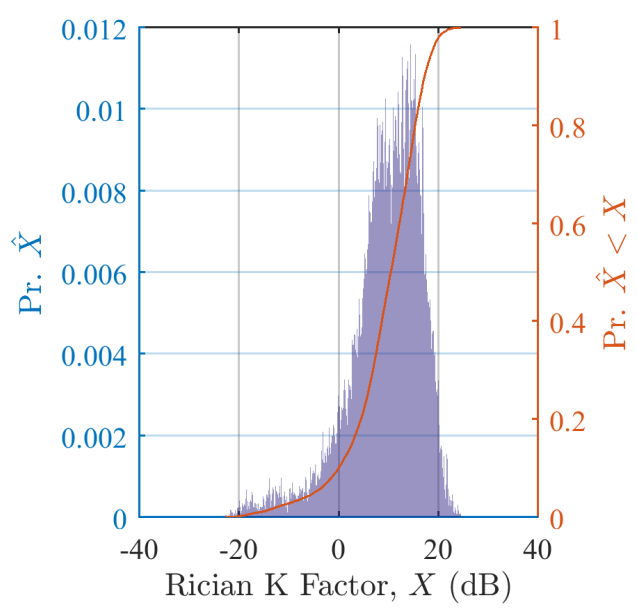

(e) Rician K-factor

Figure A.4.39: Channel characterizations for Tx2 2G Boiler 3115Vpol run1 pp channel stats 


\section{A.4.40 Tx2 2G Boiler 3115Vpol run2 pp.mat}

Table A.120: Measurement Parameters

\begin{tabular}{l|c}
\hline Parameter & Value \\
\hline \hline Mat File & Tx2 2G Boiler 3115Vpol run2 \\
Frequency (GHz) & 2.245 \\
Location & Ooulder CUP Day 2 (2016-02-10) at Bould \\
RX Antenna & Oorn Antenna, V Pol \\
RX Antenna Gain & 9.260 \\
TX Antenna & 1.780 \\
TX Antenna Gain & 4.000 \\
TX Power, Watts & 200.000 \\
PN Oversample Factor &
\end{tabular}

Table A.121: Statistics of Channel Estimates. Outliers are removed using a significance test of 0.95.

\begin{tabular}{l||c|c|c|c||c|c||l}
\hline Estimate & $\min (\cdot)$ & $\operatorname{median}(\cdot)$ & $\operatorname{mean}(\cdot)$ & $\max (\cdot)$ & $\operatorname{std}(\cdot)$ & $\operatorname{mad}(\cdot)$ & outlier info \\
\hline \hline$K(\mathrm{~dB})$, NLOS & -23.5 & 10.9 & 9.1 & 26.9 & 8.4 & 6.3 & N: 10347 \\
\hline$K(\mathrm{~dB})$, LOS & 4.5 & 13.8 & 13.8 & 26.9 & 3.7 & 3.0 & N: 6842 \\
\hline$\tau(\mathrm{ns})$ & 8.9 & 89.0 & 94.1 & 224.0 & 46.6 & 38.2 & N: 10347 \\
\hline$S(\mathrm{~ns})$ & 5.5 & 34.1 & 34.5 & 85.1 & 16.9 & 14.4 & N: 10347 \\
\hline
\end{tabular}

Table A.122: Statistics of Channel Estimates. Outliers are not removed. N indicates the total number of samples in the population. Outliers are provided at 10 and 20 times $\sigma$.

\begin{tabular}{l||c|c|c|c||c|c||l}
\hline Estimate & $\min (\cdot)$ & $\operatorname{median}(\cdot)$ & $\operatorname{mean}(\cdot)$ & $\max (\cdot)$ & $\operatorname{std}(\cdot)$ & $\operatorname{mad}(\cdot)$ & outlier info \\
\hline \hline$K(\mathrm{~dB})$, NLOS & -23.5 & 10.9 & 9.1 & 26.9 & 8.4 & 6.3 & N: 10347 \\
\hline$K(\mathrm{~dB})$, LOS & 4.5 & 13.8 & 13.8 & 26.9 & 3.7 & 3.0 & N: 6842 \\
\hline$\tau(\mathrm{ns})$ & 8.9 & 89.0 & 94.1 & 224.0 & 46.6 & 38.2 & N: 10347 \\
\hline$S(\mathrm{~ns})$ & 5.5 & 34.1 & 34.5 & 85.1 & 16.9 & 14.4 & N: 10347 \\
\hline
\end{tabular}




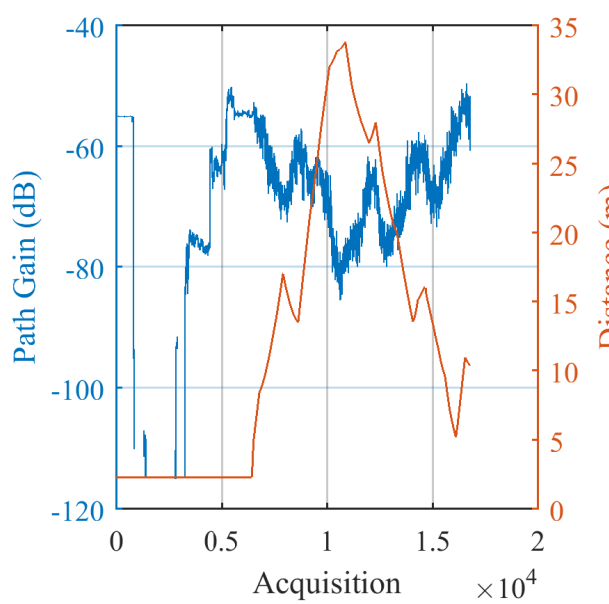

(a) Path Gain versus Acquisition Order

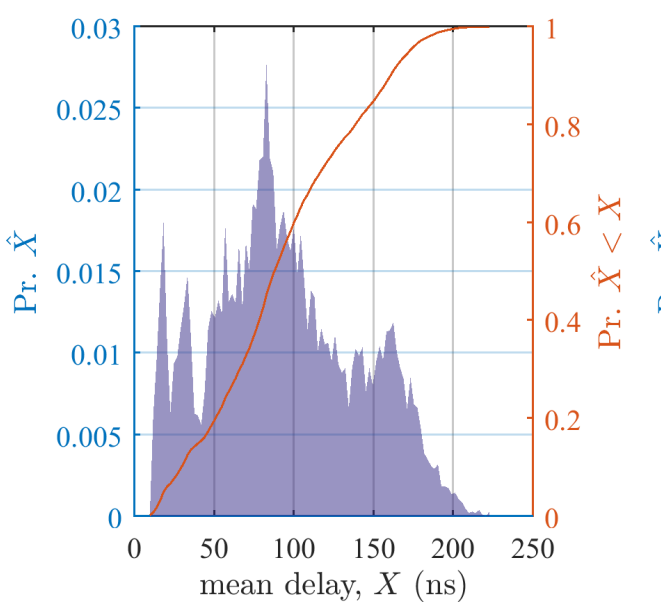

(c) Power Delay

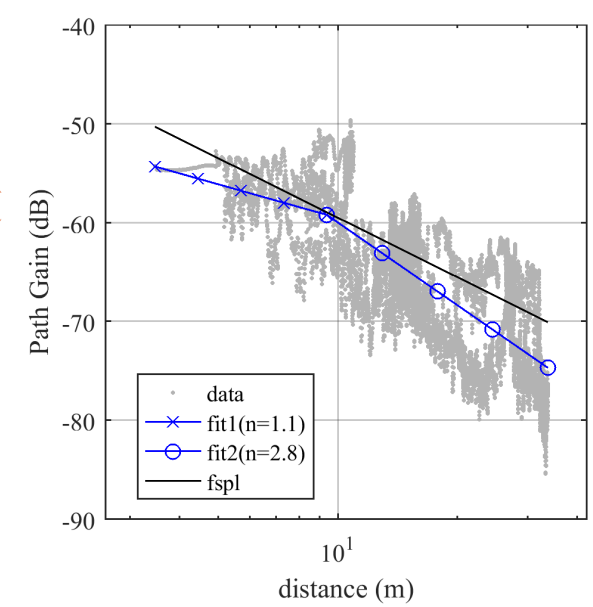

(b) Path Loss versus Distance

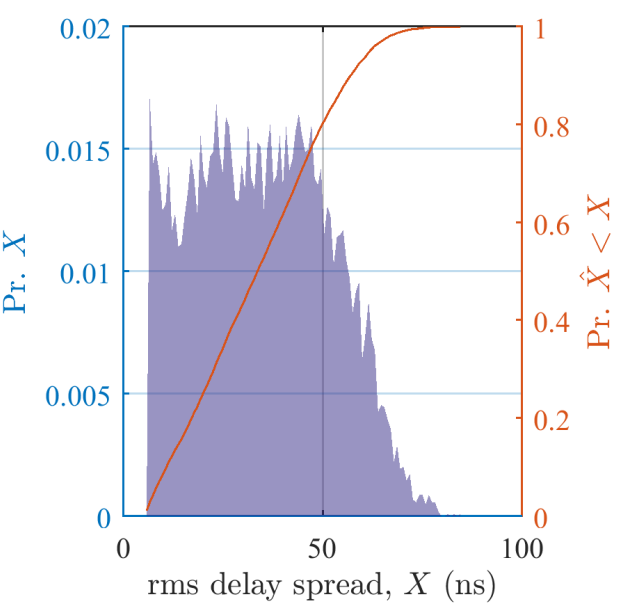

(d) Power Delay Spread

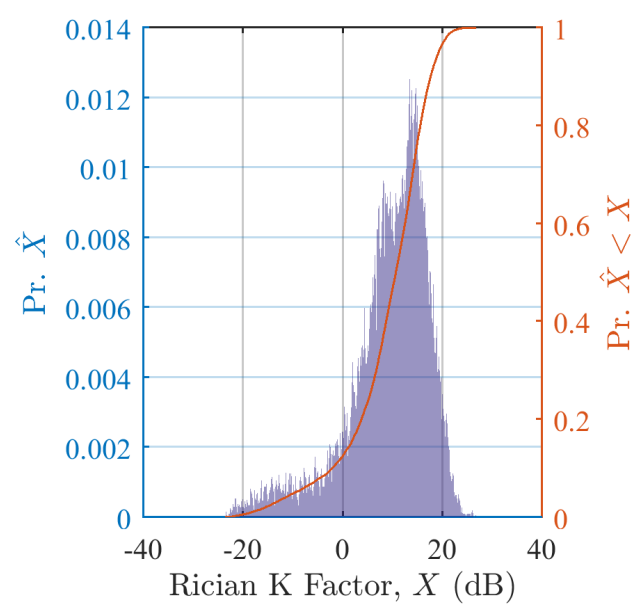

(e) Rician K-factor

Figure A.4.40: Channel characterizations for Tx2 2G Boiler 3115Vpol run2 pp channel stats 


\section{A.4.41 Tx2 2G Boiler Vpol run2 pp.mat}

Table A.123: Measurement Parameters

\begin{tabular}{l|c}
\hline Parameter & Value \\
\hline \hline Mat File & Tx2 2G Boiler Vpol run2_pp.mat \\
Frequency (GHz) & 2.245 \\
Location & Boulder CUP Day 1 (2016-02-09) at Boulder CUP Boiler Room \\
RX Antenna & Omni-directional, V Pol \\
RX Antenna Gain & -4.200 \\
TX Antenna & Omni-directional, V Pol \\
TX Antenna Gain & 2.900 \\
TX Power, Watts & 2.300 \\
PN Oversample Factor & 4.000 \\
Sample rate, MHz & 200.000
\end{tabular}

Table A.124: Statistics of Channel Estimates. Outliers are removed using a significance test of 0.95.

\begin{tabular}{l||c|c|c|c||c|c||l}
\hline Estimate & $\min (\cdot)$ & $\operatorname{median}(\cdot)$ & $\operatorname{mean}(\cdot)$ & $\max (\cdot)$ & $\operatorname{std}(\cdot)$ & $\operatorname{mad}(\cdot)$ & outlier info \\
\hline \hline$K(\mathrm{~dB})$, NLOS & -23.3 & 9.6 & 8.0 & 26.4 & 8.8 & 6.6 & N: 11315 \\
\hline$K(\mathrm{~dB})$, LOS & 5.1 & 13.2 & 13.4 & 26.4 & 3.7 & 3.1 & N: 6782 \\
\hline$\tau(\mathrm{ns})$ & 7.5 & 87.9 & 89.0 & 228.1 & 49.0 & 41.1 & N: 11315 \\
\hline$S(\mathrm{~ns})$ & 5.1 & 34.8 & 35.3 & 93.2 & 16.7 & 13.8 & N: 11315 \\
\hline
\end{tabular}

Table A.125: Statistics of Channel Estimates. Outliers are not removed. N indicates the total number of samples in the population. Outliers are provided at 10 and 20 times $\sigma$.

\begin{tabular}{l||c|c|c|c||c|c||l}
\hline Estimate & $\min (\cdot)$ & $\operatorname{median}(\cdot)$ & $\operatorname{mean}(\cdot)$ & $\max (\cdot)$ & $\operatorname{std}(\cdot)$ & $\operatorname{mad}(\cdot)$ & outlier info \\
\hline \hline$K(\mathrm{~dB})$, NLOS & -23.3 & 9.6 & 8.0 & 26.4 & 8.8 & 6.6 & N: 11315 \\
\hline$K(\mathrm{~dB})$, LOS & 5.1 & 13.2 & 13.4 & 26.4 & 3.7 & 3.1 & N: 6782 \\
\hline$\tau(\mathrm{ns})$ & 7.5 & 87.9 & 89.0 & 228.1 & 49.0 & 41.1 & N: 11315 \\
\hline$S(\mathrm{~ns})$ & 5.1 & 34.8 & 35.3 & 93.2 & 16.7 & 13.8 & N: 11315 \\
\hline
\end{tabular}




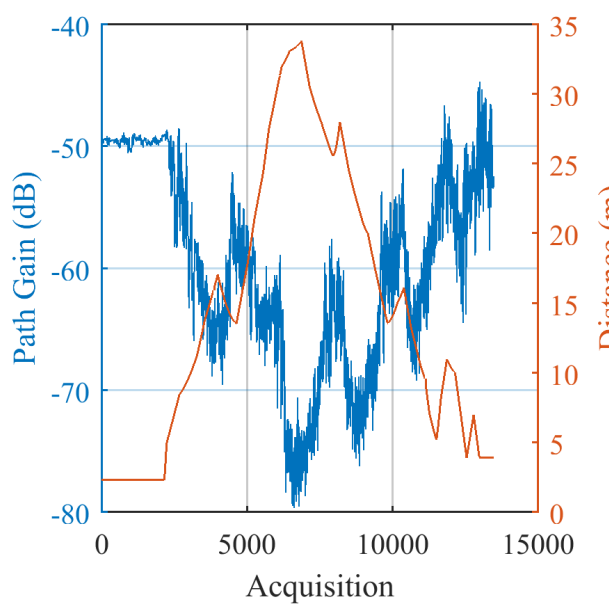

(a) Path Gain versus Acquisition Order

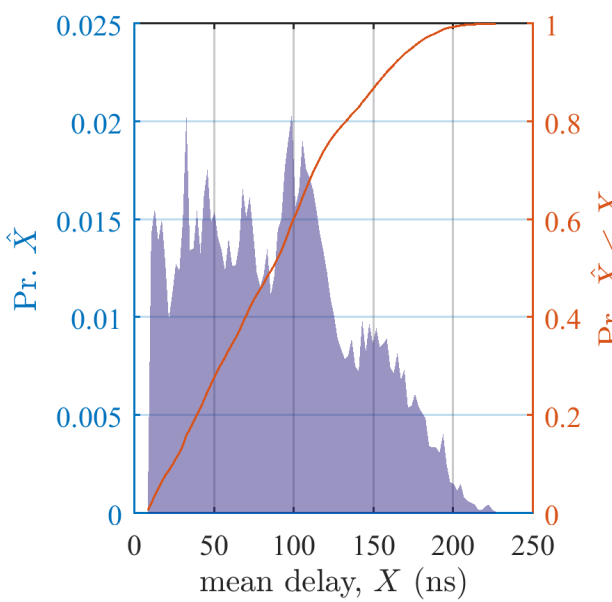

(c) Power Delay

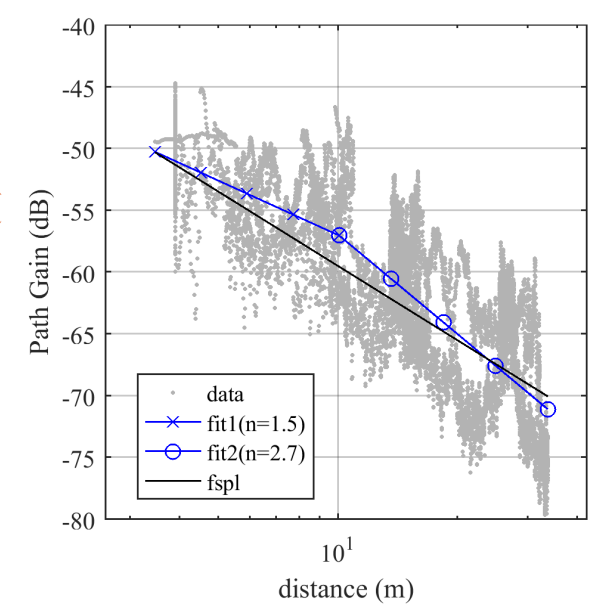

(b) Path Loss versus Distance

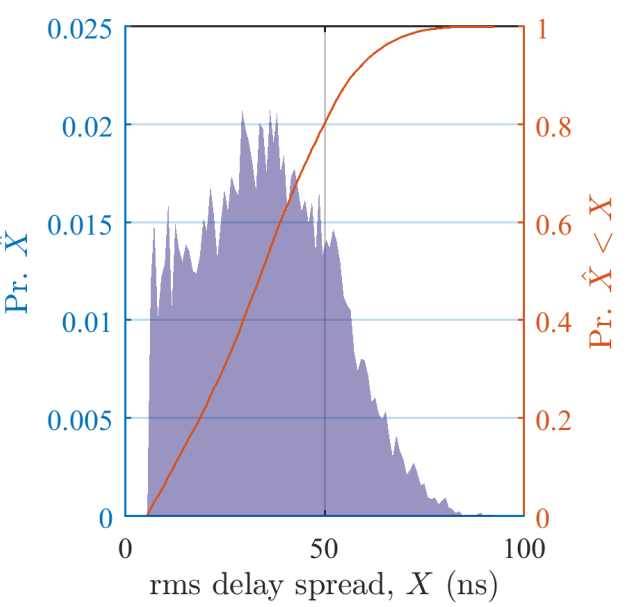

(d) Power Delay Spread

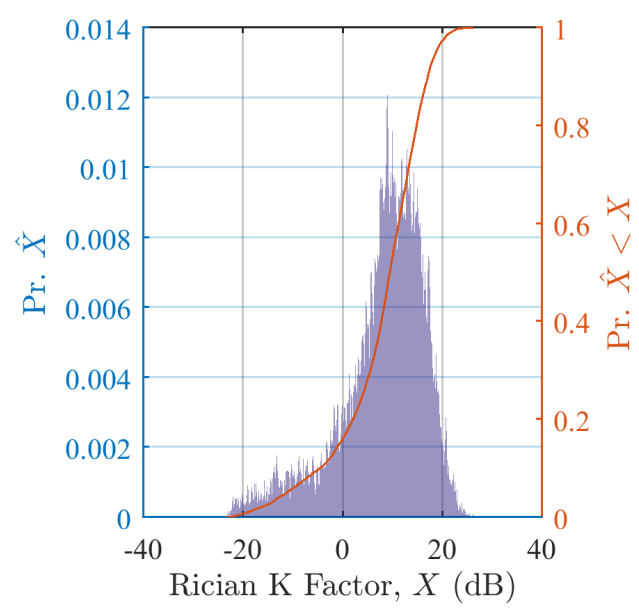

(e) Rician K-factor

Figure A.4.41: Channel characterizations for Tx2 2G Boiler Vpol run2 pp channel stats 


\section{A.4.42 Tx2 2G Boiler Vpol run3 pp.mat}

Table A.126: Measurement Parameters

\begin{tabular}{l|c}
\hline Parameter & Value \\
\hline \hline Mat File & Tx2 2G Boiler Vpol run3_pp.mat \\
Frequency $(\mathrm{GHz})$ & 2.245 \\
Location & Boulder CUP Day 1 (2016-02-09) at Boulder CUP Boiler Room \\
RX Antenna & Omni-directional, V Pol \\
RX Antenna Gain & -4.200 \\
TX Antenna & Omni-directional, V Pol \\
TX Antenna Gain & 2.900 \\
TX Power, Watts & 2.300 \\
PN Oversample Factor & 4.000 \\
Sample rate, MHz & 200.000
\end{tabular}

Table A.127: Statistics of Channel Estimates. Outliers are removed using a significance test of 0.95.

\begin{tabular}{l||c|c|c|c||c|c||l}
\hline Estimate & $\min (\cdot)$ & $\operatorname{median}(\cdot)$ & $\operatorname{mean}(\cdot)$ & $\max (\cdot)$ & $\operatorname{std}(\cdot)$ & $\operatorname{mad}(\cdot)$ & outlier info \\
\hline \hline$K(\mathrm{~dB})$, NLOS & -24.2 & 9.7 & 8.3 & 26.5 & 8.2 & 6.2 & N: 9299 \\
\hline$K(\mathrm{~dB})$, LOS & 3.2 & 13.1 & 13.3 & 26.5 & 3.6 & 3.0 & N: 5761 \\
\hline$\tau(\mathrm{ns})$ & 2.5 & 89.4 & 90.6 & 234.9 & 49.7 & 41.0 & N: 9299 \\
\hline$S(\mathrm{~ns})$ & 3.8 & 34.8 & 35.4 & 94.9 & 17.1 & 14.4 & N: 9299 \\
\hline
\end{tabular}

Table A.128: Statistics of Channel Estimates. Outliers are not removed. N indicates the total number of samples in the population. Outliers are provided at 10 and 20 times $\sigma$.

\begin{tabular}{l||c|c|c|c||c|c||l}
\hline Estimate & $\min (\cdot)$ & $\operatorname{median}(\cdot)$ & $\operatorname{mean}(\cdot)$ & $\max (\cdot)$ & $\operatorname{std}(\cdot)$ & $\operatorname{mad}(\cdot)$ & outlier info \\
\hline \hline$K(\mathrm{~dB})$, NLOS & -24.2 & 9.7 & 8.3 & 26.5 & 8.2 & 6.2 & N: 9299 \\
\hline$K(\mathrm{~dB})$, LOS & 3.2 & 13.1 & 13.3 & 26.5 & 3.6 & 3.0 & N: 5761 \\
\hline$\tau(\mathrm{ns})$ & 2.5 & 89.4 & 90.6 & 234.9 & 49.7 & 41.0 & N: 9299 \\
\hline$S(\mathrm{~ns})$ & 3.8 & 34.8 & 35.4 & 94.9 & 17.1 & 14.4 & N: 9299 \\
\hline
\end{tabular}




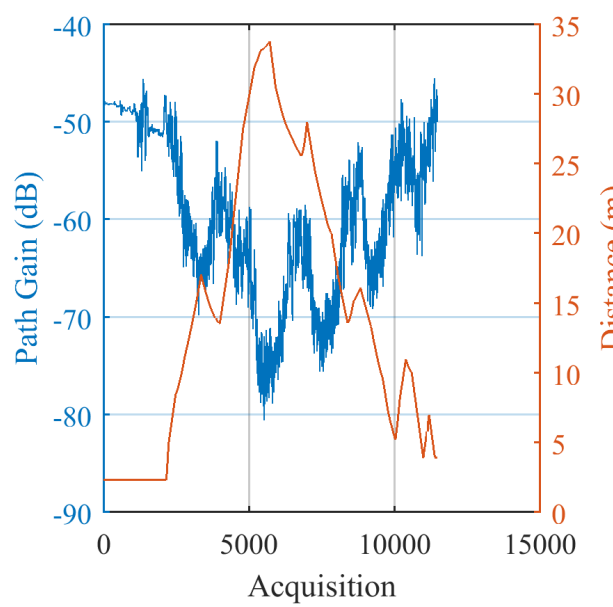

(a) Path Gain versus Acquisition Order

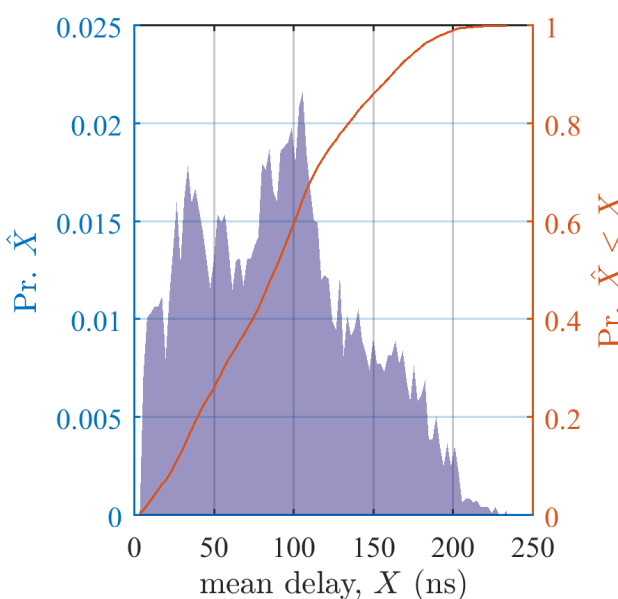

(c) Power Delay

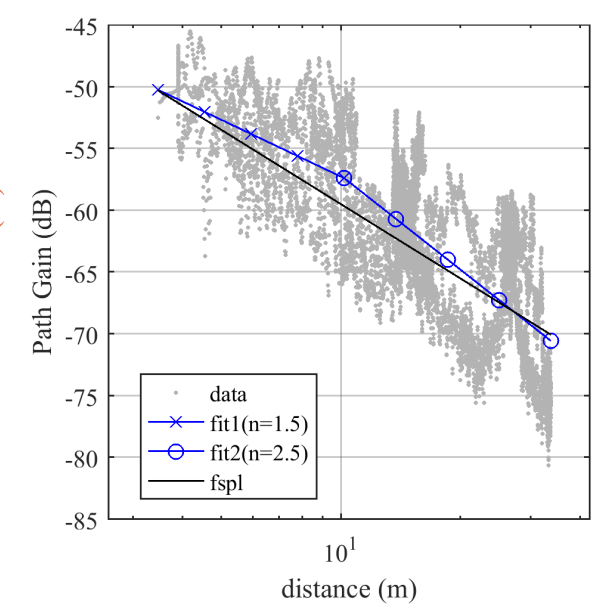

(b) Path Loss versus Distance

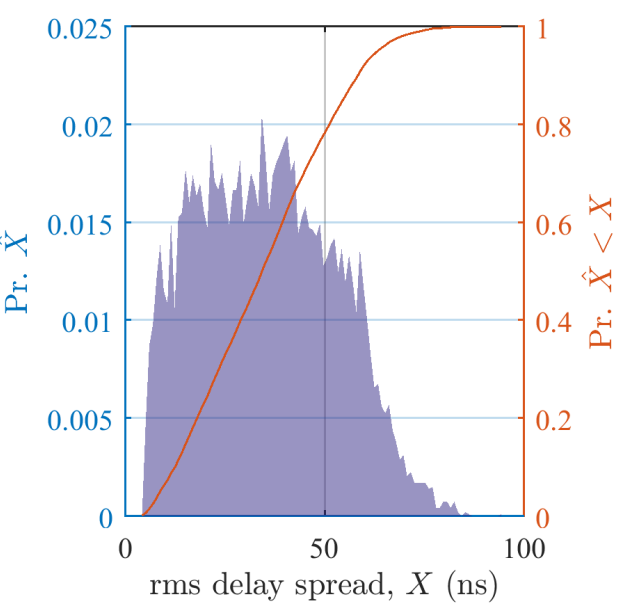

(d) Power Delay Spread

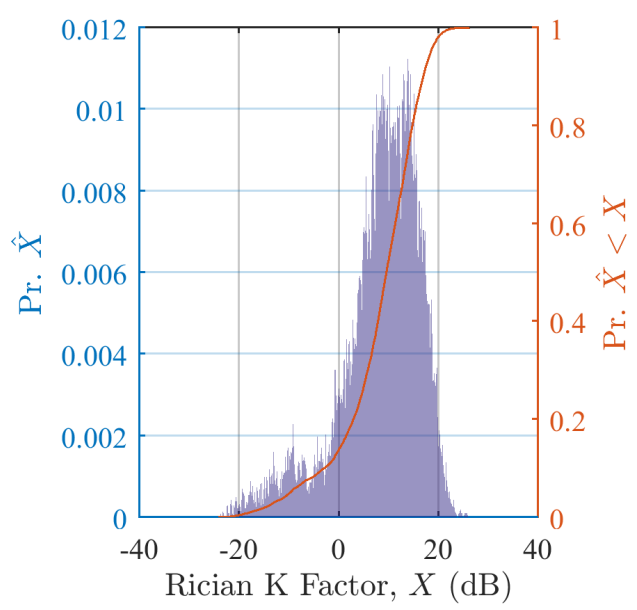

(e) Rician K-factor

Figure A.4.42: Channel characterizations for Tx2 2G Boiler Vpol run3 pp channel stats 


\section{A.4.43 Tx2 2G Boiler Vpol run4 pp.mat}

Table A.129: Measurement Parameters

\begin{tabular}{l|c}
\hline Parameter & Value \\
\hline \hline Mat File & Tx2 2G Boiler Vpol run4_pp.mat \\
Frequency $(\mathrm{GHz})$ & 2.245 \\
Location & Boulder CUP Day 1 (2016-02-09) at Boulder CUP Boiler Room \\
RX Antenna & Omni-directional, V Pol \\
RX Antenna Gain & -4.200 \\
TX Antenna & Omni-directional, V Pol \\
TX Antenna Gain & 2.900 \\
TX Power, Watts & 2.150 \\
PN Oversample Factor & 4.000 \\
Sample rate, MHz & 200.000
\end{tabular}

Table A.130: Statistics of Channel Estimates. Outliers are removed using a significance test of 0.95.

\begin{tabular}{l||c|c|c|c||c|c||l}
\hline Estimate & $\min (\cdot)$ & $\operatorname{median}(\cdot)$ & $\operatorname{mean}(\cdot)$ & $\max (\cdot)$ & $\operatorname{std}(\cdot)$ & $\operatorname{mad}(\cdot)$ & outlier info \\
\hline \hline$K(\mathrm{~dB})$, NLOS & -24.2 & 9.5 & 8.0 & 26.0 & 8.2 & 6.2 & N: 12243 \\
\hline$K(\mathrm{~dB})$, LOS & 4.6 & 12.9 & 13.1 & 26.0 & 3.4 & 2.8 & N: 7365 \\
\hline$\tau(\mathrm{ns})$ & 21.5 & 95.9 & 100.6 & 244.0 & 46.2 & 38.0 & N: 12243 \\
\hline$S(\mathrm{~ns})$ & 5.7 & 35.9 & 36.8 & 89.6 & 16.7 & 13.9 & N: 12243 \\
\hline
\end{tabular}

Table A.131: Statistics of Channel Estimates. Outliers are not removed. N indicates the total number of samples in the population. Outliers are provided at 10 and 20 times $\sigma$.

\begin{tabular}{l||c|c|c|c||c|c||l}
\hline Estimate & $\min (\cdot)$ & $\operatorname{median}(\cdot)$ & $\operatorname{mean}(\cdot)$ & $\max (\cdot)$ & $\operatorname{std}(\cdot)$ & $\operatorname{mad}(\cdot)$ & outlier info \\
\hline \hline$K(\mathrm{~dB})$, NLOS & -24.2 & 9.5 & 8.0 & 26.0 & 8.2 & 6.2 & N: 12243 \\
\hline$K(\mathrm{~dB})$, LOS & 4.6 & 12.9 & 13.1 & 26.0 & 3.4 & 2.8 & N: 7365 \\
\hline$\tau(\mathrm{ns})$ & 21.5 & 95.9 & 100.6 & 244.0 & 46.2 & 38.0 & N: 12243 \\
\hline$S(\mathrm{~ns})$ & 5.7 & 35.9 & 36.8 & 89.6 & 16.7 & 13.9 & N: 12243 \\
\hline
\end{tabular}




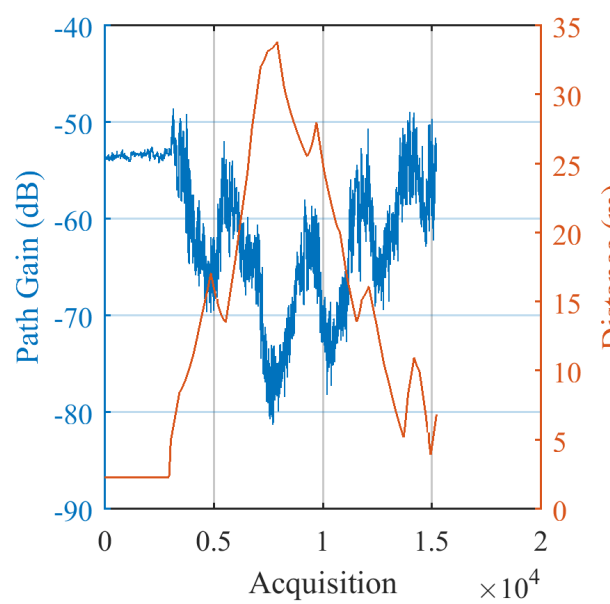

(a) Path Gain versus Acquisition Order

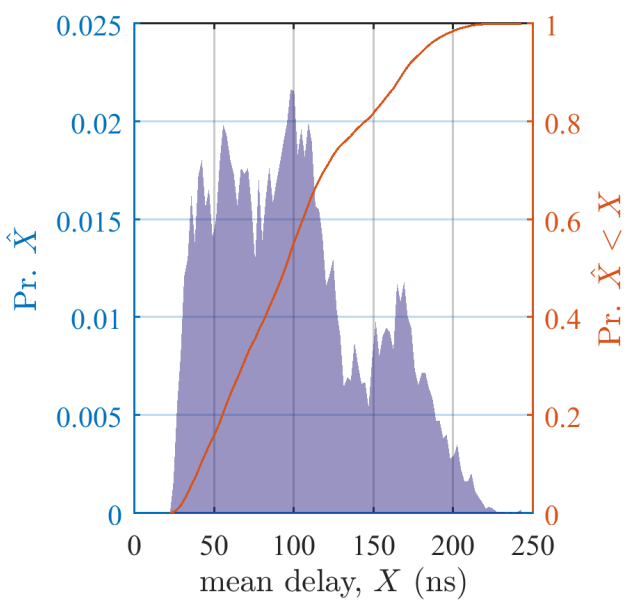

(c) Power Delay

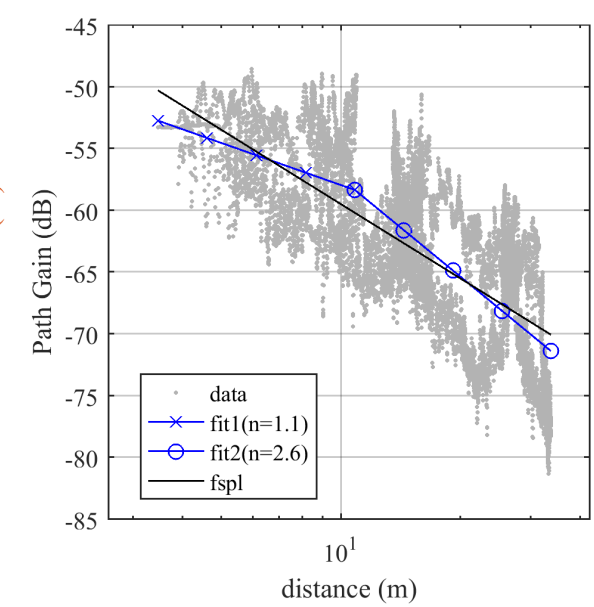

(b) Path Loss versus Distance

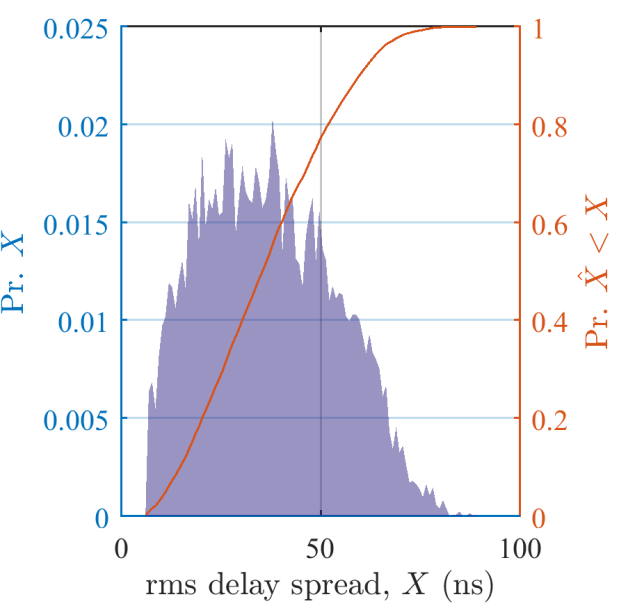

(d) Power Delay Spread

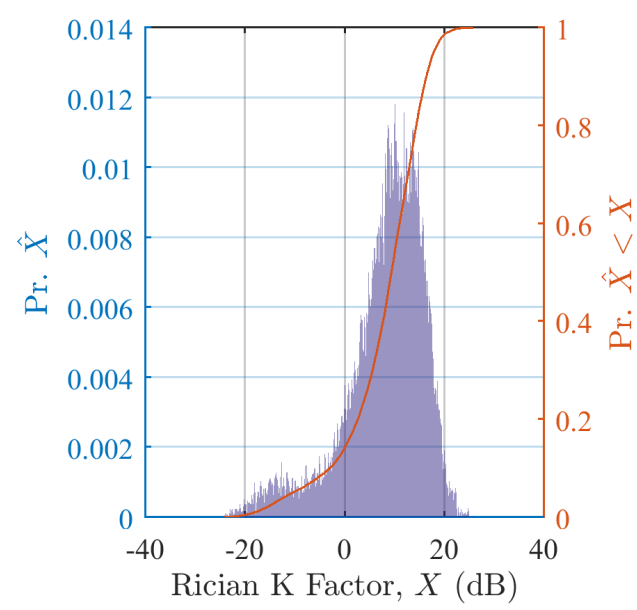

(e) Rician K-factor

Figure A.4.43: Channel characterizations for Tx2 2G Boiler Vpol run4 pp channel stats 


\section{A.4.44 Tx2 5G Boiler 3115Vpol run1 pp.mat}

Table A.132: Measurement Parameters

\begin{tabular}{l|c}
\hline Parameter & Value \\
\hline \hline Mat File & Tx2 5G Boiler 3115Vpol run1_pp.mat \\
Frequency $(\mathrm{GHz})$ & 5.400 \\
Location & Boulder CUP Day 4 (2016-02-12) at Boulder CUP Boiler Room \\
RX Antenna & Omni-directional, V Pol \\
RX Antenna Gain & -3.500 \\
TX Antenna & Horn Antenna, V Pol \\
TX Antenna Gain & 10.320 \\
TX Power, Watts & 3.190 \\
PN Oversample Factor & 4.000 \\
Sample rate, MHz & 200.000
\end{tabular}

Table A.133: Statistics of Channel Estimates. Outliers are removed using a significance test of 0.95.

\begin{tabular}{l||c|c|c|c||c|c||l}
\hline Estimate & $\min (\cdot)$ & $\operatorname{median}(\cdot)$ & $\operatorname{mean}(\cdot)$ & $\max (\cdot)$ & $\operatorname{std}(\cdot)$ & $\operatorname{mad}(\cdot)$ & outlier info \\
\hline \hline$K(\mathrm{~dB})$, NLOS & -25.3 & 8.9 & 5.1 & 27.7 & 13.0 & 10.4 & N: 8795 \\
\hline$K(\mathrm{~dB})$, LOS & 2.4 & 14.1 & 14.2 & 27.7 & 4.2 & 3.5 & N: 4893 \\
\hline$\tau(\mathrm{ns})$ & 0.5 & 80.5 & 87.0 & 237.0 & 51.8 & 44.1 & N: 8795 \\
\hline$S(\mathrm{~ns})$ & 1.5 & 35.2 & 35.4 & 92.4 & 18.1 & 15.3 & N: 8795 \\
\hline
\end{tabular}

Table A.134: Statistics of Channel Estimates. Outliers are not removed. N indicates the total number of samples in the population. Outliers are provided at 10 and 20 times $\sigma$.

\begin{tabular}{l||c|c|c|c||c|c||l}
\hline Estimate & $\min (\cdot)$ & $\operatorname{median}(\cdot)$ & $\operatorname{mean}(\cdot)$ & $\max (\cdot)$ & $\operatorname{std}(\cdot)$ & $\operatorname{mad}(\cdot)$ & outlier info \\
\hline \hline$K(\mathrm{~dB})$, NLOS & -25.3 & 8.9 & 5.1 & 27.7 & 13.0 & 10.4 & N: 8795 \\
\hline$K(\mathrm{~dB})$, LOS & 2.4 & 14.1 & 14.2 & 27.7 & 4.2 & 3.5 & N: 4893 \\
\hline$\tau(\mathrm{ns})$ & 0.5 & 80.5 & 87.0 & 237.0 & 51.8 & 44.1 & N: 8795 \\
\hline$S(\mathrm{~ns})$ & 1.5 & 35.2 & 35.4 & 92.4 & 18.1 & 15.3 & N: 8795 \\
\hline
\end{tabular}




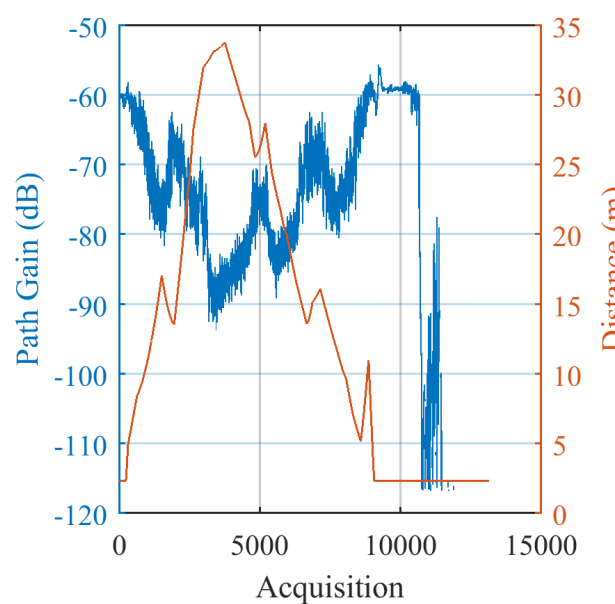

(a) Path Gain versus Acquisition Order

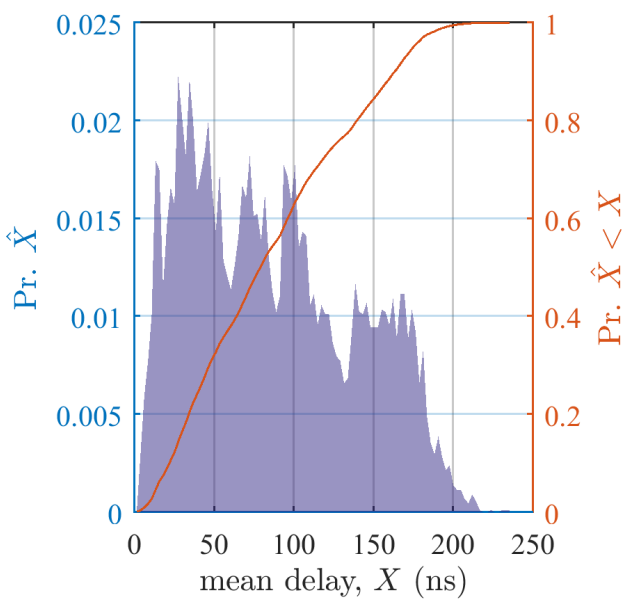

(c) Power Delay

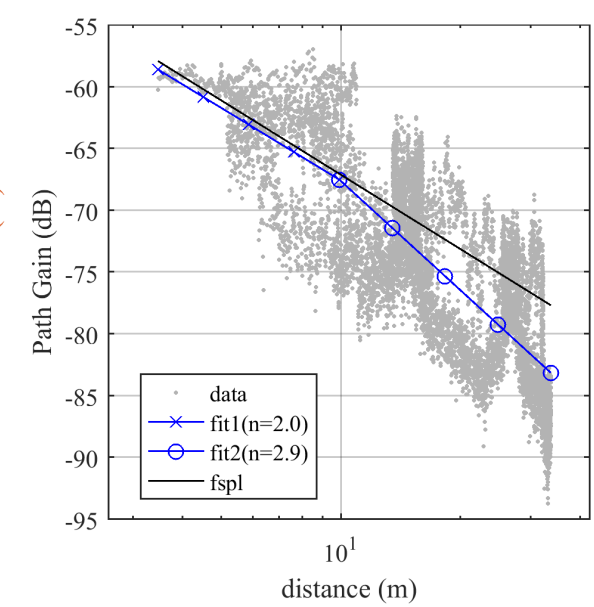

(b) Path Loss versus Distance

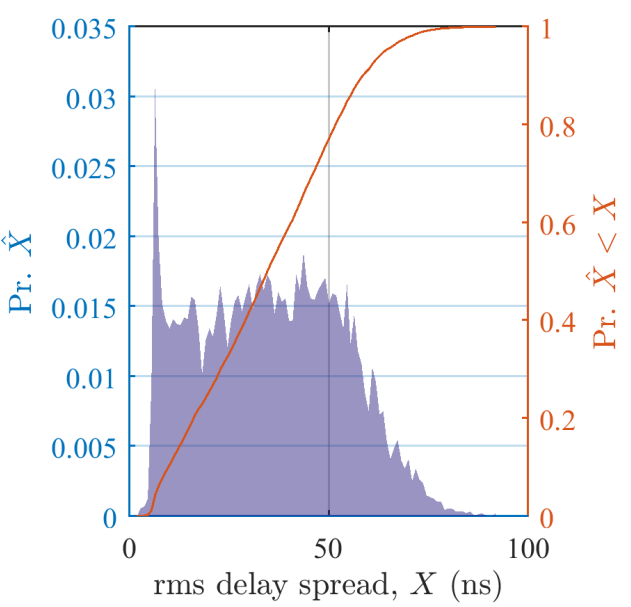

(d) Power Delay Spread

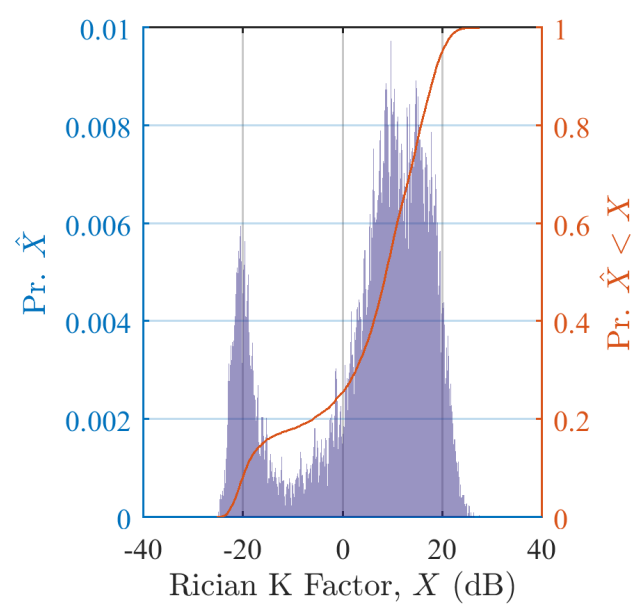

(e) Rician K-factor

Figure A.4.44: Channel characterizations for Tx2 5G Boiler 3115Vpol run1 pp channel stats 


\section{A.4.45 Tx2 5G Boiler 3115Xpol run1 pp.mat}

Table A.135: Measurement Parameters

\begin{tabular}{l|c}
\hline Parameter & Value \\
\hline \hline Mat File & Tx2 5G Boiler 3115Xpol run1_pp.mat \\
Frequency $(\mathrm{GHz})$ & 5.400 \\
Location & Boulder CUP Day 4 (2016-02-12) at Boulder CUP Boiler Room \\
RX Antenna & Omni-directional, Cross Pol \\
RX Antenna Gain & -3.500 \\
TX Antenna & Horn Antenna, X Pol \\
TX Antenna Gain & 10.320 \\
TX Power, Watts & 3.190 \\
PN Oversample Factor & 4.000 \\
Sample rate, MHz & 200.000
\end{tabular}

Table A.136: Statistics of Channel Estimates. Outliers are removed using a significance test of 0.95.

\begin{tabular}{l||c|c|c|c||c|c||l}
\hline Estimate & $\min (\cdot)$ & $\operatorname{median}(\cdot)$ & $\operatorname{mean}(\cdot)$ & $\max (\cdot)$ & $\operatorname{std}(\cdot)$ & $\operatorname{mad}(\cdot)$ & outlier info \\
\hline \hline$K(\mathrm{~dB})$, NLOS & -25.4 & -11.4 & -6.6 & 21.3 & 13.3 & 12.3 & N: 10947 \\
\hline$K(\mathrm{~dB})$, LOS & 3.7 & 11.6 & 11.9 & 21.3 & 3.0 & 2.4 & N: 2318 \\
\hline$\tau(\mathrm{ns})$ & 37.5 & 145.2 & 146.1 & 263.3 & 44.2 & 36.6 & N: 10947 \\
\hline$S(\mathrm{~ns})$ & 6.0 & 48.7 & 48.4 & 97.6 & 13.2 & 10.6 & N: 10947 \\
\hline
\end{tabular}

Table A.137: Statistics of Channel Estimates. Outliers are not removed. N indicates the total number of samples in the population. Outliers are provided at 10 and 20 times $\sigma$.

\begin{tabular}{l||c|c|c|c||c|c||l}
\hline Estimate & $\min (\cdot)$ & $\operatorname{median}(\cdot)$ & $\operatorname{mean}(\cdot)$ & $\max (\cdot)$ & $\operatorname{std}(\cdot)$ & $\operatorname{mad}(\cdot)$ & outlier info \\
\hline \hline$K(\mathrm{~dB})$, NLOS & -25.4 & -11.4 & -6.6 & 21.3 & 13.3 & 12.3 & N: 10947 \\
\hline$K(\mathrm{~dB})$, LOS & 3.7 & 11.6 & 11.9 & 21.3 & 3.0 & 2.4 & N: 2318 \\
\hline$\tau(\mathrm{ns})$ & 37.5 & 145.2 & 146.1 & 263.3 & 44.2 & 36.6 & N: 10947 \\
\hline$S(\mathrm{~ns})$ & 6.0 & 48.7 & 48.4 & 97.6 & 13.2 & 10.6 & N: 10947 \\
\hline
\end{tabular}




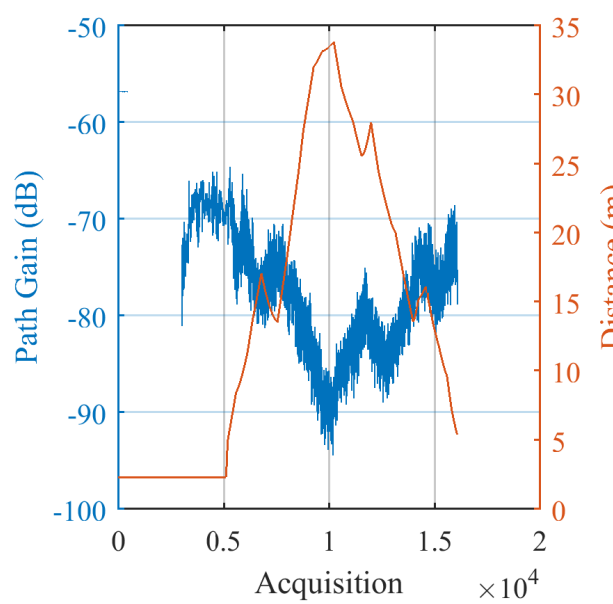

(a) Path Gain versus Acquisition Order

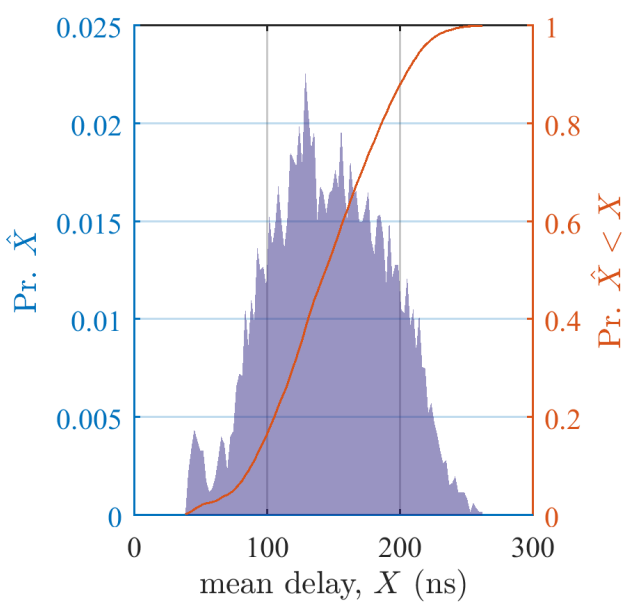

(c) Power Delay

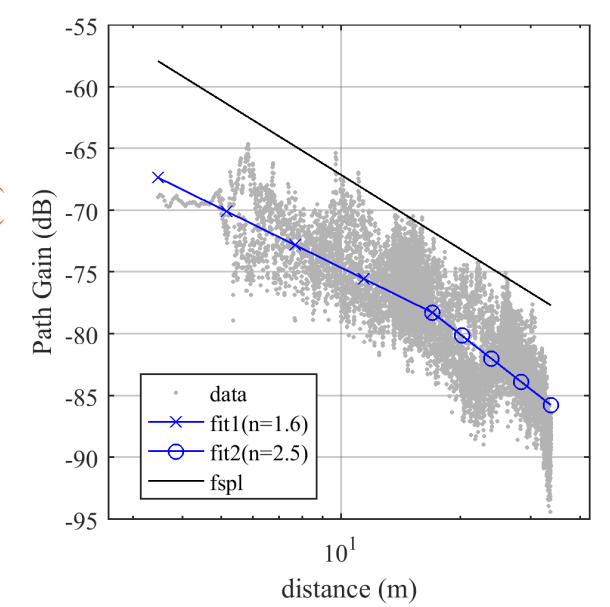

(b) Path Loss versus Distance

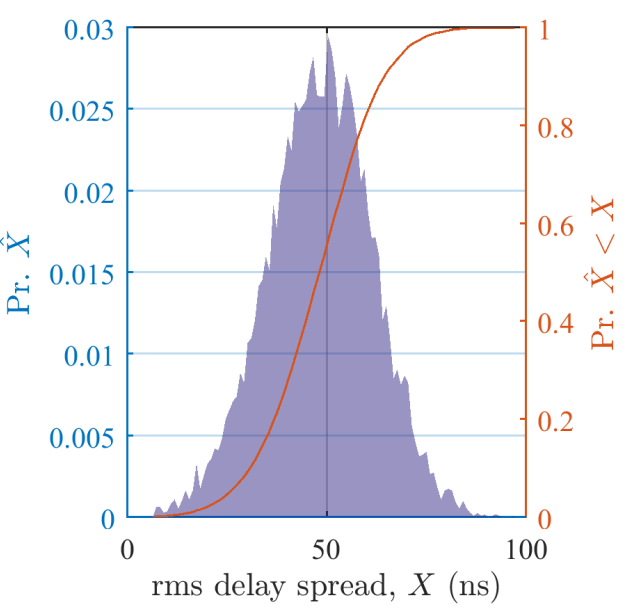

(d) Power Delay Spread

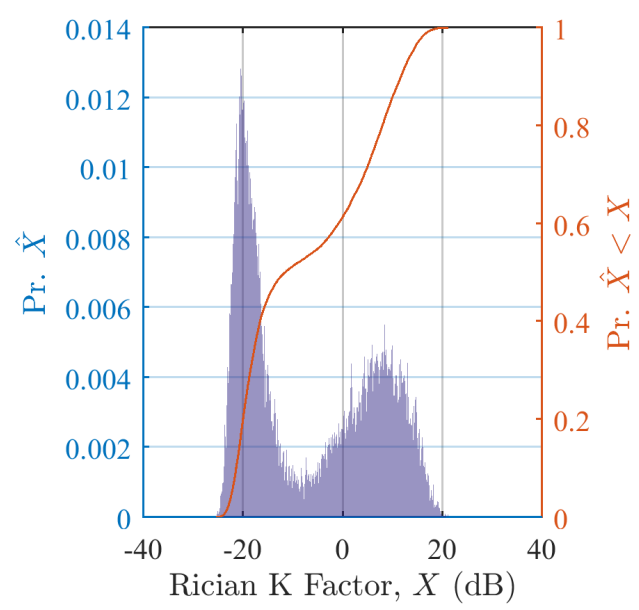

(e) Rician K-factor

Figure A.4.45: Channel characterizations for Tx2 5G Boiler 3115Xpol run1 pp channel stats 


\section{A.4.46 Tx2 5G Boiler Vpol run1 pp.mat}

Table A.138: Measurement Parameters

\begin{tabular}{l|c}
\hline Parameter & Value \\
\hline \hline Mat File & Tx2 5G Boiler Vpol run1_pp.mat \\
Frequency $(\mathrm{GHz})$ & 5.400 \\
Location & Boulder CUP Day 4 (2016-02-12) at Boulder CUP Boiler Room \\
RX Antenna & Omni-directional, V Pol \\
RX Antenna Gain & -3.500 \\
TX Antenna & Omni-directional, V Pol \\
TX Antenna Gain & 3.600 \\
TX Power, Watts & 3.180 \\
PN Oversample Factor & 4.000 \\
Sample rate, MHz & 200.000
\end{tabular}

Table A.139: Statistics of Channel Estimates. Outliers are removed using a significance test of 0.95.

\begin{tabular}{l||c|c|c|c||c|c||l}
\hline Estimate & $\min (\cdot)$ & $\operatorname{median}(\cdot)$ & $\operatorname{mean}(\cdot)$ & $\max (\cdot)$ & $\operatorname{std}(\cdot)$ & $\operatorname{mad}(\cdot)$ & outlier info \\
\hline \hline$K(\mathrm{~dB})$, NLOS & -24.9 & 2.7 & -1.0 & 27.5 & 15.8 & 14.9 & N: 12978 \\
\hline$K(\mathrm{~dB})$, LOS & 0.0 & 15.0 & 14.6 & 27.5 & 4.1 & 3.3 & N: 5762 \\
\hline$\tau(\mathrm{ns})$ & 0.0 & 89.1 & 92.7 & 340.0 & 56.2 & 46.7 & N: 13278 \\
\hline$S(\mathrm{~ns})$ & 0.0 & 30.4 & 30.9 & 92.5 & 19.3 & 16.1 & N: 14423 \\
\hline
\end{tabular}

Table A.140: Statistics of Channel Estimates. Outliers are not removed. N indicates the total number of samples in the population. Outliers are provided at 10 and 20 times $\sigma$.

\begin{tabular}{l||c|c|c|c||c|c||l}
\hline Estimate & $\min (\cdot)$ & $\operatorname{median}(\cdot)$ & $\operatorname{mean}(\cdot)$ & $\max (\cdot)$ & $\operatorname{std}(\cdot)$ & $\operatorname{mad}(\cdot)$ & outlier info \\
\hline \hline$K(\mathrm{~dB})$, NLOS & -24.9 & 2.7 & -1.0 & 27.5 & 15.8 & 14.9 & $\mathrm{~N}: 12978$ \\
\hline$K(\mathrm{~dB})$, LOS & 0.0 & 15.0 & 14.6 & 27.5 & 4.1 & 3.3 & $\mathrm{~N}: 5762$ \\
\hline$\tau(\mathrm{ns})$ & 0.0 & 97.0 & 1562.7 & 32680.0 & 5345.8 & 2671.8 & $\mathrm{~N}: 14629$ \\
\hline$S(\mathrm{~ns})$ & 0.0 & 30.8 & 104.7 & 13353.4 & 736.5 & 145.5 & $\mathrm{~N}: 14629$ \\
& & & & & & & $10 \sigma(7469.6): 54$ \\
\hline
\end{tabular}




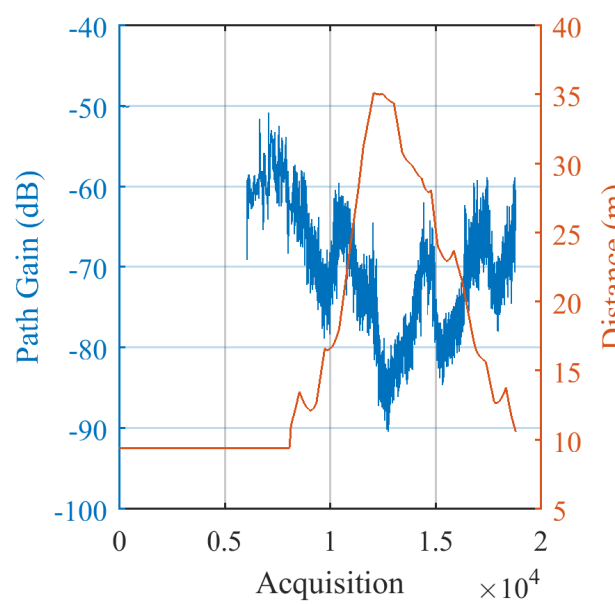

(a) Path Gain versus Acquisition Order

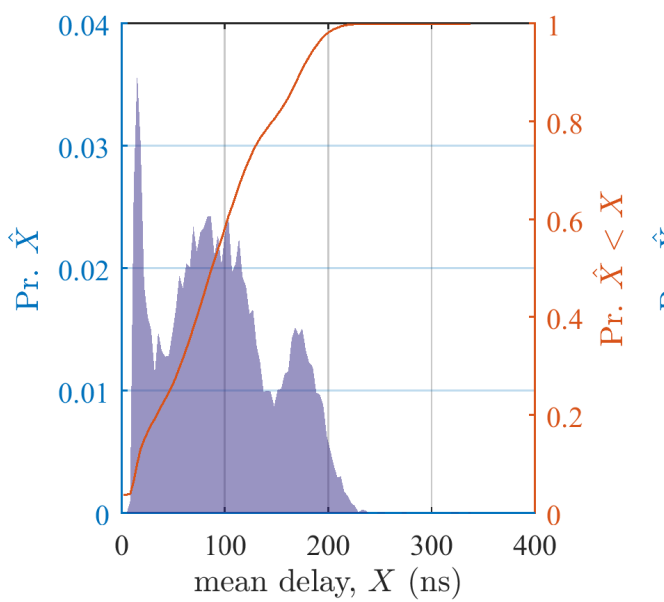

(c) Power Delay

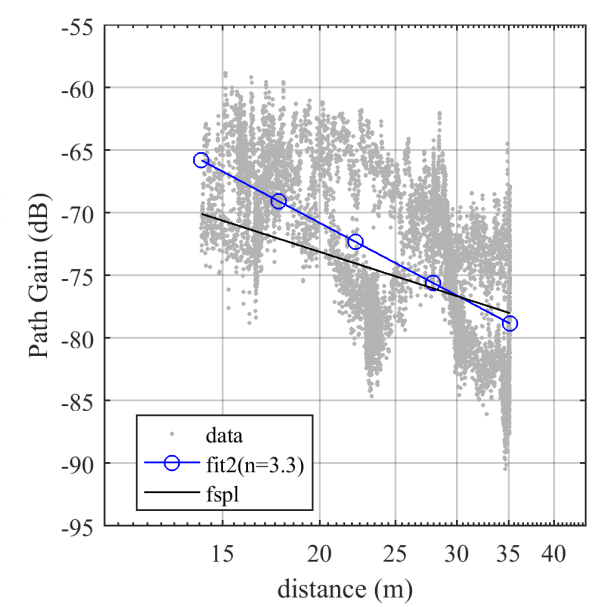

(b) Path Loss versus Distance

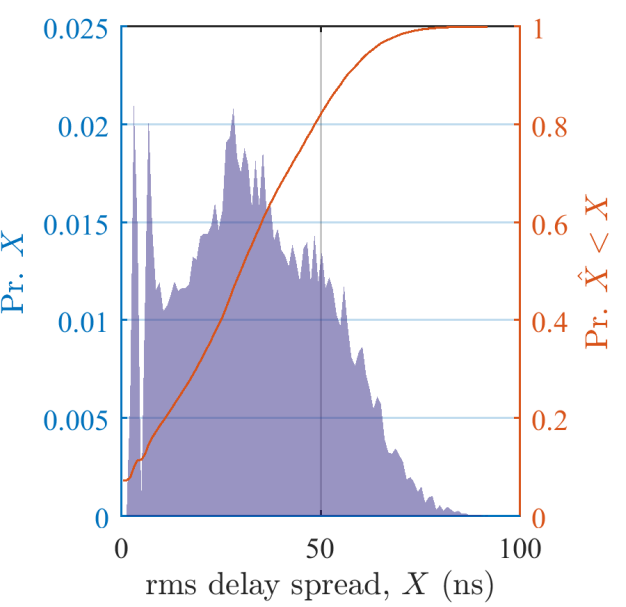

(d) Power Delay Spread

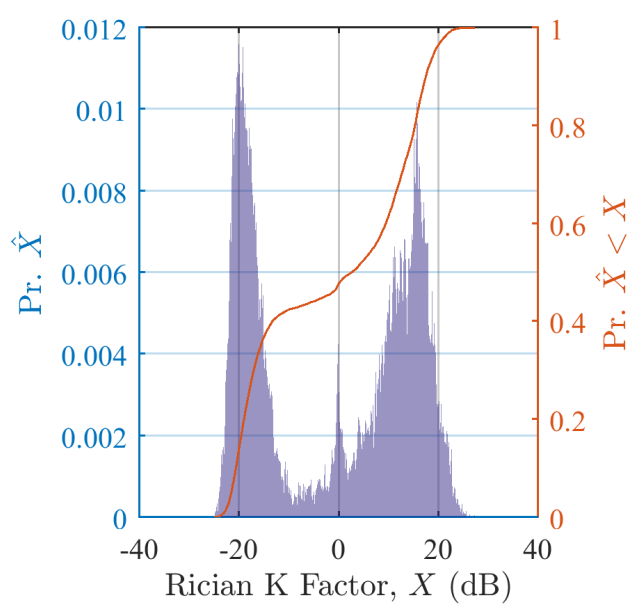

(e) Rician K-factor

Figure A.4.46: Channel characterizations for Tx2 5G Boiler Vpol run1 pp channel stats 


\section{A.4.47 Tx2 5G Boiler Xpol run1 pp.mat}

Table A.141: Measurement Parameters

\begin{tabular}{l|c}
\hline Parameter & Value \\
\hline \hline Mat File & Tx2 5G Boiler Xpol run1_pp.mat \\
Frequency (GHz) & 5.400 \\
Location & Boulder CUP Day 4 (2016-02-12) at Boulder CUP Boiler Room \\
RX Antenna & Omni-directional, Cross Pol \\
RX Antenna Gain & -3.500 \\
TX Antenna & Omni-directional, X Pol \\
TX Antenna Gain & 3.600 \\
TX Power, Watts & 3.180 \\
PN Oversample Factor & 4.000 \\
Sample rate, MHz & 200.000
\end{tabular}

Table A.142: Statistics of Channel Estimates. Outliers are removed using a significance test of 0.95.

\begin{tabular}{l||c|c|c|c||c|c||l}
\hline Estimate & $\min (\cdot)$ & $\operatorname{median}(\cdot)$ & $\operatorname{mean}(\cdot)$ & $\max (\cdot)$ & $\operatorname{std}(\cdot)$ & $\operatorname{mad}(\cdot)$ & outlier info \\
\hline \hline$K(\mathrm{~dB})$, NLOS & -25.2 & -14.8 & -10.1 & 18.0 & 10.9 & 9.3 & N: 11952 \\
\hline$K(\mathrm{~dB})$, LOS & 5.5 & 9.8 & 10.3 & 18.0 & 2.4 & 1.9 & N: 1245 \\
\hline$\tau(\mathrm{ns})$ & 44.4 & 139.7 & 139.9 & 272.5 & 43.5 & 36.2 & N: 11952 \\
\hline$S(\mathrm{~ns})$ & 12.6 & 51.5 & 51.4 & 101.9 & 12.2 & 9.7 & N: 11951 \\
\hline
\end{tabular}

Table A.143: Statistics of Channel Estimates. Outliers are not removed. N indicates the total number of samples in the population. Outliers are provided at 10 and 20 times $\sigma$.

\begin{tabular}{l||c|c|c|c||c|c||l}
\hline Estimate & $\min (\cdot)$ & $\operatorname{median}(\cdot)$ & $\operatorname{mean}(\cdot)$ & $\max (\cdot)$ & $\operatorname{std}(\cdot)$ & $\operatorname{mad}(\cdot)$ & outlier info \\
\hline \hline$K(\mathrm{~dB})$, NLOS & -25.2 & -14.8 & -10.1 & 18.0 & 10.9 & 9.3 & N: 11952 \\
\hline$K(\mathrm{~dB})$, LOS & 5.5 & 9.8 & 10.3 & 18.0 & 2.4 & 1.9 & N: 1245 \\
\hline$\tau(\mathrm{ns})$ & 44.4 & 139.7 & 139.9 & 272.5 & 43.5 & 36.2 & N: 11952 \\
\hline$S(\mathrm{~ns})$ & 12.6 & 51.5 & 51.4 & 107.7 & 12.2 & 9.7 & N: 11952 \\
\hline
\end{tabular}




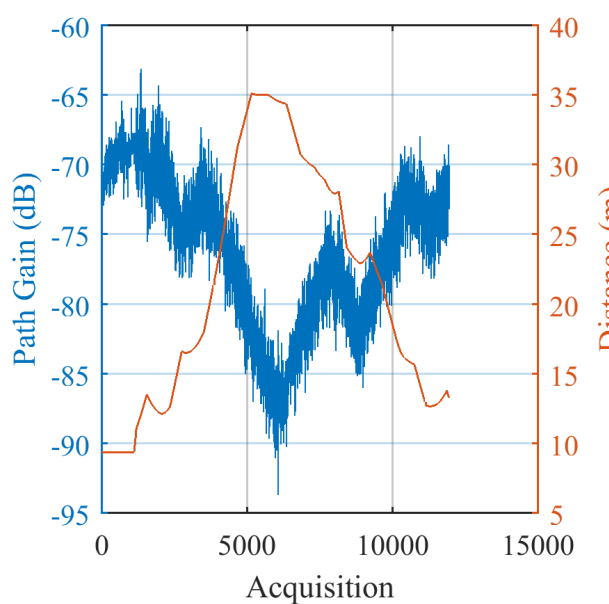

(a) Path Gain versus Acquisition Order

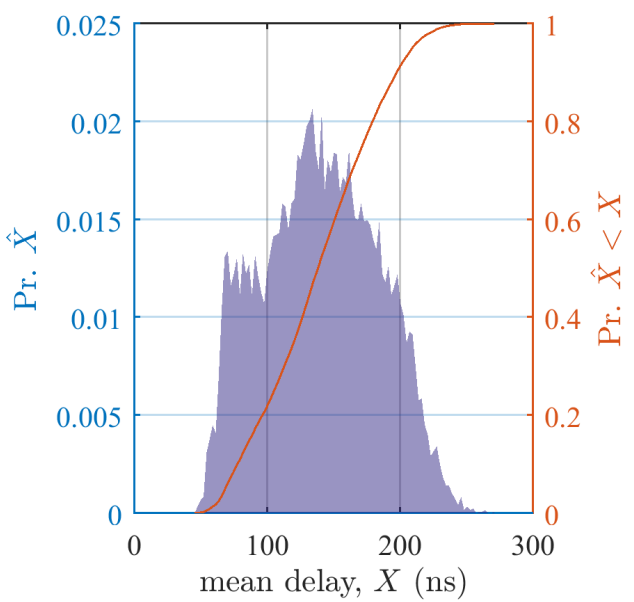

(c) Power Delay

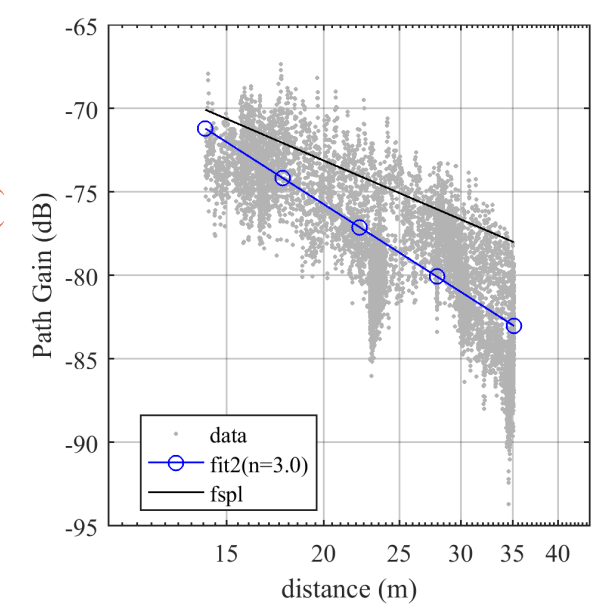

(b) Path Loss versus Distance

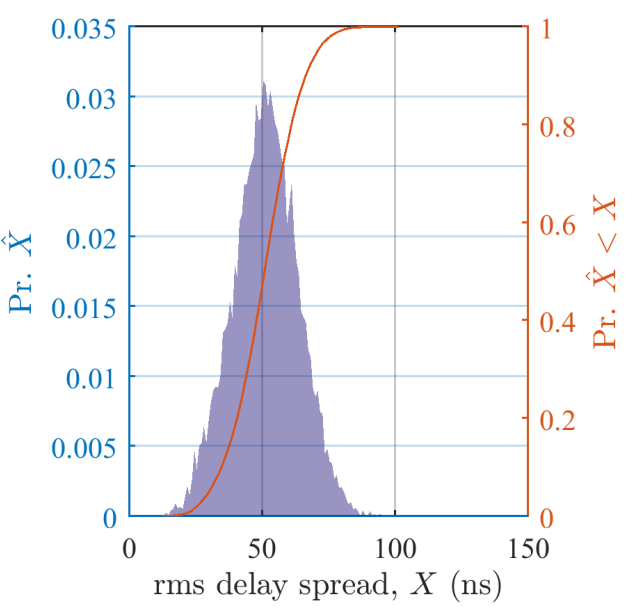

(d) Power Delay Spread

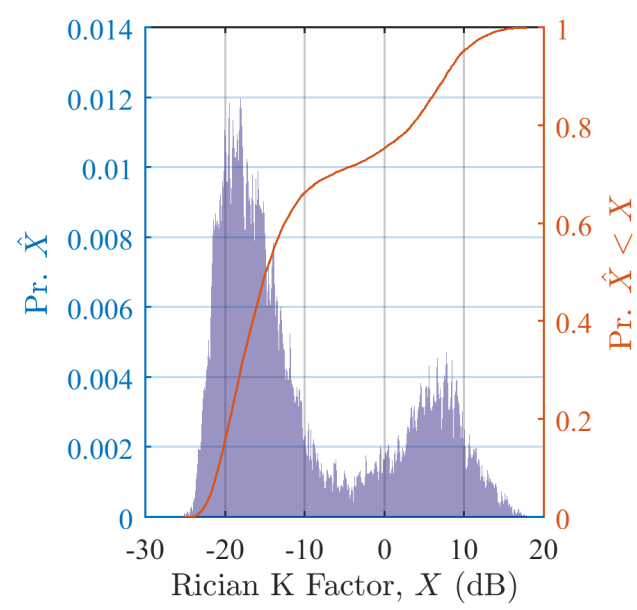

(e) Rician K-factor

Figure A.4.47: Channel characterizations for Tx2 5G Boiler Xpol run1 pp channel stats 


\section{A.4.48 Tx2 5G Boiler 3115Vpol run1 pp.mat}

Table A.144: Measurement Parameters

\begin{tabular}{l|c}
\hline Parameter & Value \\
\hline \hline Mat File & Tx2_5G_Boiler_3115Vpol_run1_pp.mat \\
Frequency $(\mathrm{GHz})$ & 5.400 \\
Location & Boulder CUP Day 4 (2016-02-12) at Boulder CUP Boiler Room \\
RX Antenna & Omni-directional, V Pol \\
RX Antenna Gain & -3.500 \\
TX Antenna & Horn Antenna, V Pol \\
TX Antenna Gain & 10.320 \\
TX Power, Watts & 3.190 \\
PN Oversample Factor & 4.000 \\
Sample rate, MHz & 200.000
\end{tabular}

Table A.145: Statistics of Channel Estimates. Outliers are removed using a significance test of 0.95.

\begin{tabular}{l||c|c|c|c||c|c||l}
\hline Estimate & $\min (\cdot)$ & $\operatorname{median}(\cdot)$ & $\operatorname{mean}(\cdot)$ & $\max (\cdot)$ & $\operatorname{std}(\cdot)$ & $\operatorname{mad}(\cdot)$ & outlier info \\
\hline \hline$K(\mathrm{~dB})$, NLOS & -25.3 & 8.9 & 5.1 & 27.7 & 13.0 & 10.4 & N: 8795 \\
\hline$K(\mathrm{~dB})$, LOS & 2.4 & 14.1 & 14.2 & 27.7 & 4.2 & 3.5 & N: 4893 \\
\hline$\tau(\mathrm{ns})$ & 0.5 & 80.5 & 87.0 & 237.0 & 51.8 & 44.1 & N: 8795 \\
\hline$S(\mathrm{~ns})$ & 1.5 & 35.2 & 35.4 & 92.4 & 18.1 & 15.3 & N: 8795 \\
\hline
\end{tabular}

Table A.146: Statistics of Channel Estimates. Outliers are not removed. N indicates the total number of samples in the population. Outliers are provided at 10 and 20 times $\sigma$.

\begin{tabular}{l||c|c|c|c||c|c||l}
\hline Estimate & $\min (\cdot)$ & $\operatorname{median}(\cdot)$ & $\operatorname{mean}(\cdot)$ & $\max (\cdot)$ & $\operatorname{std}(\cdot)$ & $\operatorname{mad}(\cdot)$ & outlier info \\
\hline \hline$K(\mathrm{~dB})$, NLOS & -25.3 & 8.9 & 5.1 & 27.7 & 13.0 & 10.4 & N: 8795 \\
\hline$K(\mathrm{~dB})$, LOS & 2.4 & 14.1 & 14.2 & 27.7 & 4.2 & 3.5 & N: 4893 \\
\hline$\tau(\mathrm{ns})$ & 0.5 & 80.5 & 87.0 & 237.0 & 51.8 & 44.1 & N: 8795 \\
\hline$S(\mathrm{~ns})$ & 1.5 & 35.2 & 35.4 & 92.4 & 18.1 & 15.3 & N: 8795 \\
\hline
\end{tabular}




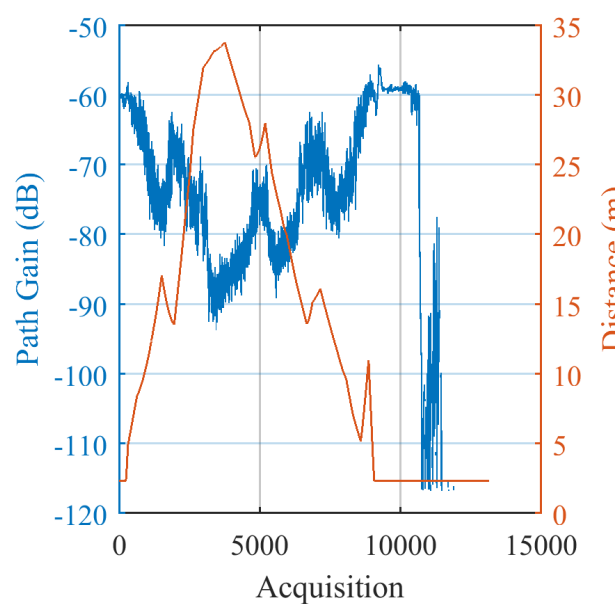

(a) Path Gain versus Acquisition Order

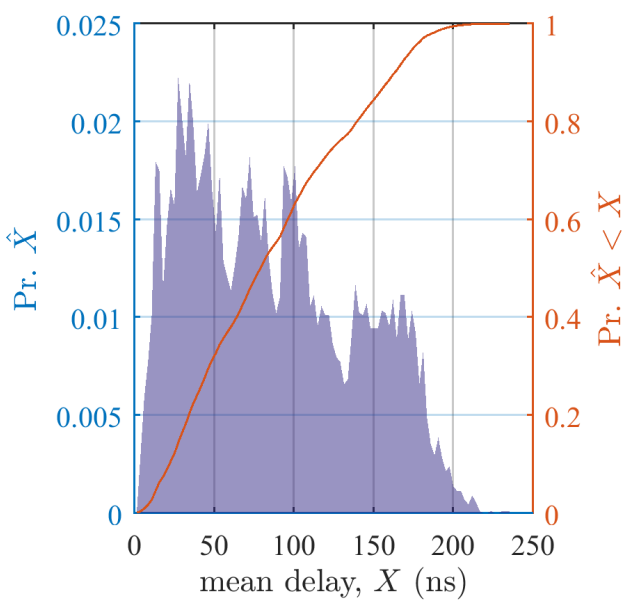

(c) Power Delay

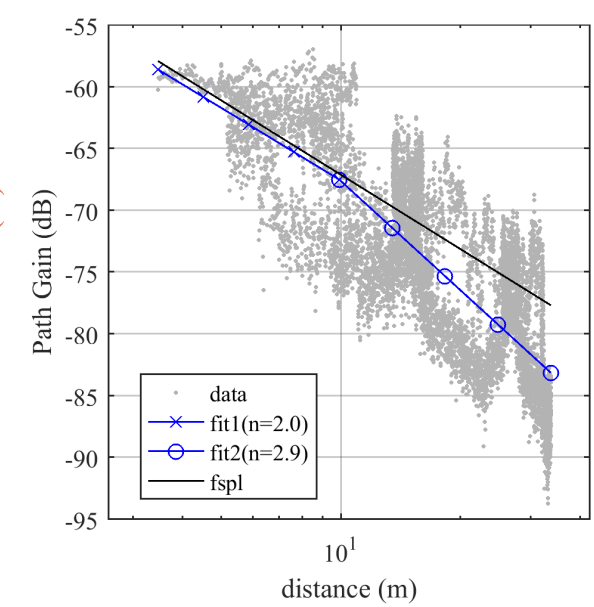

(b) Path Loss versus Distance

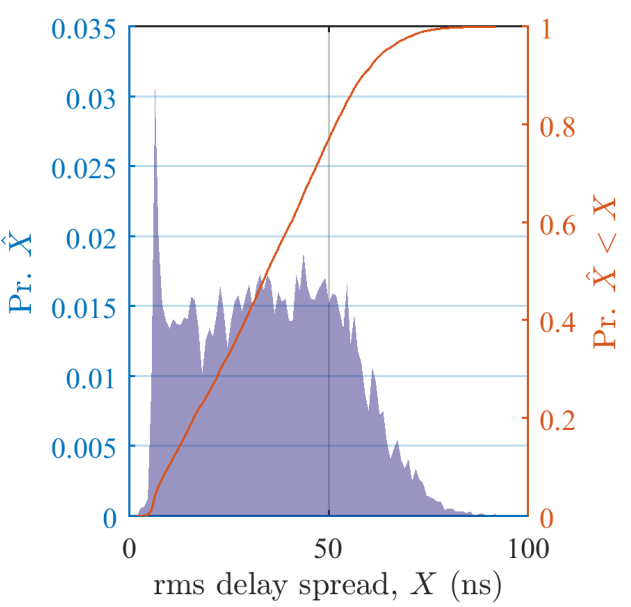

(d) Power Delay Spread

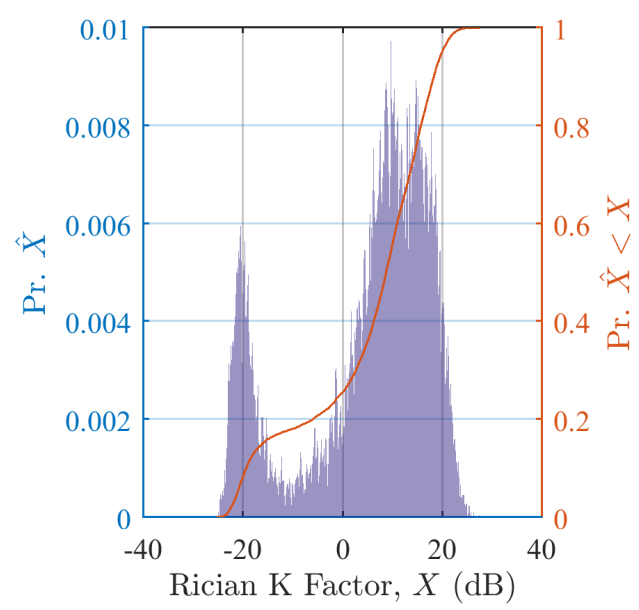

(e) Rician K-factor

Figure A.4.48: Channel characterizations for Tx2 5G Boiler 3115Vpol run1 pp channel stats 


\section{A.4.49 Tx3 2G Boiler 3115Vpol run2 pp.mat}

Table A.147: Measurement Parameters

\begin{tabular}{l|c}
\hline Parameter & Value \\
\hline \hline Mat File & Tx3 2G Boiler 3115Vpol run2 \\
Frequency (GHz) & 2.245 \\
Location & Ooulder CUP Day 2 (2016-02-10) at Bould \\
RX Antenna & Horn Antenna, V Pol \\
RX Antenna Gain & 9.260 \\
TX Antenna & 3.600 \\
TX Antenna Gain & 4.000 \\
TX Power, Watts & 200.000 \\
PN Oversample Factor &
\end{tabular}

Table A.148: Statistics of Channel Estimates. Outliers are removed using a significance test of 0.95.

\begin{tabular}{l||c|c|c|c||c|c||l}
\hline Estimate & $\min (\cdot)$ & $\operatorname{median}(\cdot)$ & $\operatorname{mean}(\cdot)$ & $\max (\cdot)$ & $\operatorname{std}(\cdot)$ & $\operatorname{mad}(\cdot)$ & outlier info \\
\hline \hline$K(\mathrm{~dB})$, NLOS & -24.6 & -3.4 & -4.4 & 18.7 & 11.0 & 10.2 & N: 15084 \\
\hline$K(\mathrm{~dB})$, LOS & 4.6 & 10.1 & 10.5 & 18.7 & 2.5 & 2.0 & N: 2855 \\
\hline$\tau(\mathrm{ns})$ & 38.8 & 96.7 & 111.7 & 235.2 & 44.8 & 39.2 & N: 15084 \\
\hline$S(\mathrm{~ns})$ & 9.2 & 39.8 & 42.2 & 102.4 & 14.4 & 12.4 & N: 15084 \\
\hline
\end{tabular}

Table A.149: Statistics of Channel Estimates. Outliers are not removed. N indicates the total number of samples in the population. Outliers are provided at 10 and 20 times $\sigma$.

\begin{tabular}{l||c|c|c|c||c|c||l}
\hline Estimate & $\min (\cdot)$ & $\operatorname{median}(\cdot)$ & $\operatorname{mean}(\cdot)$ & $\max (\cdot)$ & $\operatorname{std}(\cdot)$ & $\operatorname{mad}(\cdot)$ & outlier info \\
\hline \hline$K(\mathrm{~dB})$, NLOS & -24.6 & -3.4 & -4.4 & 18.7 & 11.0 & 10.2 & N: 15084 \\
\hline$K(\mathrm{~dB})$, LOS & 4.6 & 10.1 & 10.5 & 18.7 & 2.5 & 2.0 & N: 2855 \\
\hline$\tau(\mathrm{ns})$ & 38.8 & 96.7 & 111.7 & 235.2 & 44.8 & 39.2 & N: 15084 \\
\hline$S(\mathrm{~ns})$ & 9.2 & 39.8 & 42.2 & 102.4 & 14.4 & 12.4 & N: 15084 \\
\hline
\end{tabular}




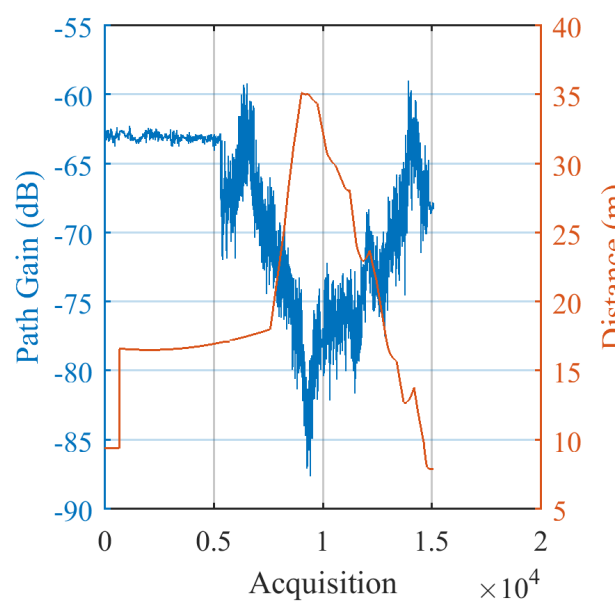

(a) Path Gain versus Acquisition Order

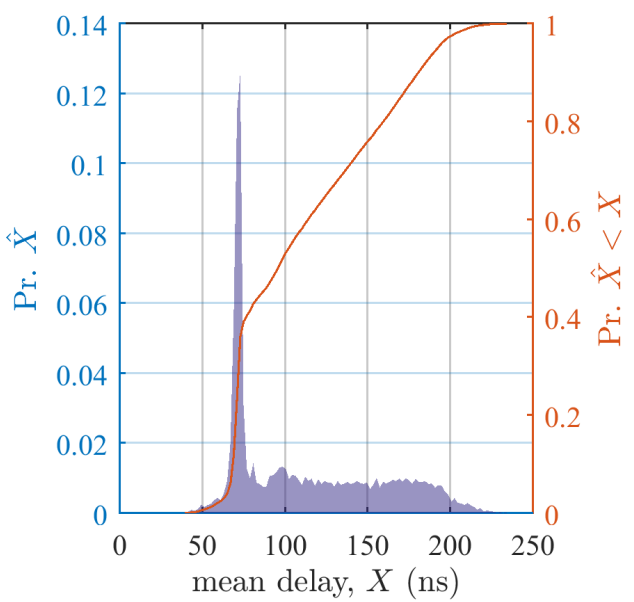

(c) Power Delay

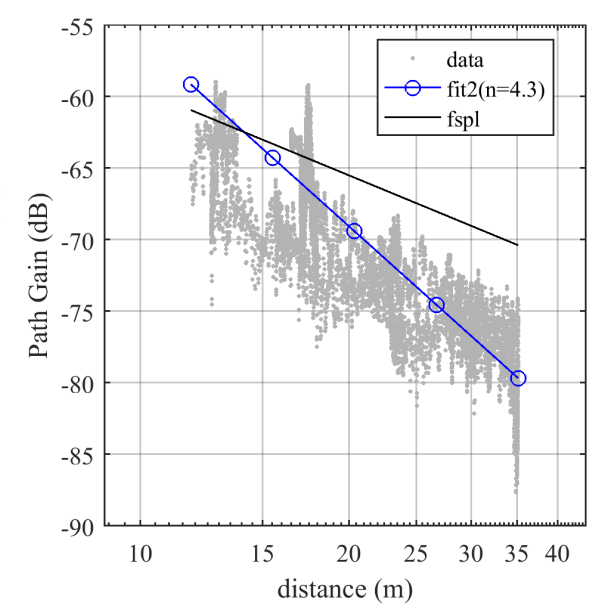

(b) Path Loss versus Distance

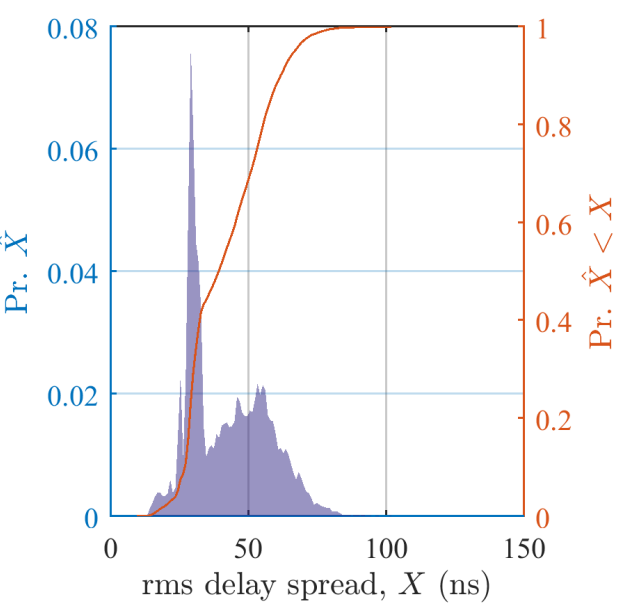

(d) Power Delay Spread

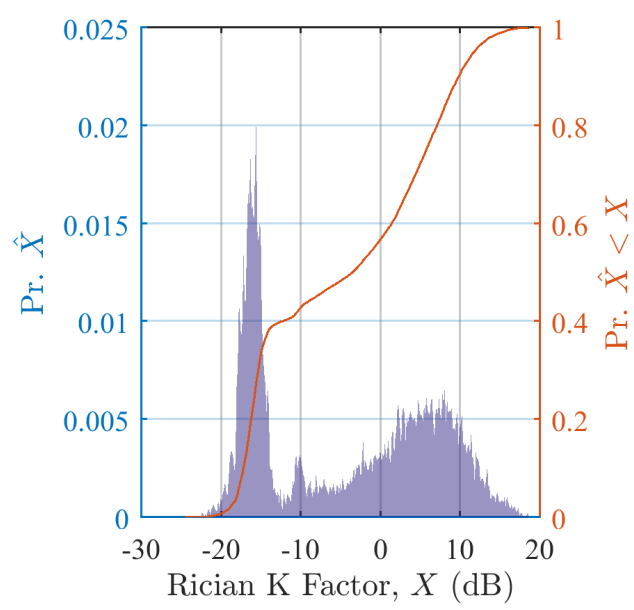

(e) Rician K-factor

Figure A.4.49: Channel characterizations for Tx3 2G Boiler 3115Vpol run2 pp channel stats 


\section{A.4.50 Tx3 5G 3115Xpol run1 pp.mat}

Table A.150: Measurement Parameters

\begin{tabular}{l|c}
\hline Parameter & Value \\
\hline \hline Mat File & Tx3 5G 3115Xpol run1_pp.mat \\
Frequency $(\mathrm{GHz})$ & 5.400
\end{tabular}

Location

RX Antenna

RX Antenna Gain

TX Antenna

TX Antenna Gain

TX Power, Watts

PN Oversample Factor

Sample rate, $\mathrm{MHz}$
Boulder CUP Day 4 (2016-02-12) at Boulder CUP Boiler Room

Omni-directional, Cross Pol

$-3.500$

Horn Antenna, V Pol

10.320

3.160

4.000

200.000

Table A.151: Statistics of Channel Estimates. Outliers are removed using a significance test of 0.95 .

\begin{tabular}{l||c|c|c|c||c|c||l}
\hline Estimate & $\min (\cdot)$ & $\operatorname{median}(\cdot)$ & $\operatorname{mean}(\cdot)$ & $\max (\cdot)$ & $\operatorname{std}(\cdot)$ & $\operatorname{mad}(\cdot)$ & outlier info \\
\hline \hline$K(\mathrm{~dB})$, NLOS & -25.4 & -8.6 & -6.5 & 19.8 & 11.6 & 10.5 & N: 12636 \\
\hline$K(\mathrm{~dB})$, LOS & 5.3 & 10.0 & 10.3 & 19.8 & 2.6 & 2.1 & N: 2059 \\
\hline$\tau(\mathrm{ns})$ & 35.6 & 151.7 & 151.8 & 268.5 & 40.8 & 33.4 & N: 12636 \\
\hline$S(\mathrm{~ns})$ & 12.8 & 54.9 & 54.0 & 106.7 & 12.4 & 9.8 & N: 12636 \\
\hline
\end{tabular}

Table A.152: Statistics of Channel Estimates. Outliers are not removed. N indicates the total number of samples in the population. Outliers are provided at 10 and 20 times $\sigma$.

\begin{tabular}{l||c|c|c|c||c|c||l}
\hline Estimate & $\min (\cdot)$ & $\operatorname{median}(\cdot)$ & $\operatorname{mean}(\cdot)$ & $\max (\cdot)$ & $\operatorname{std}(\cdot)$ & $\operatorname{mad}(\cdot)$ & outlier info \\
\hline \hline$K(\mathrm{~dB})$, NLOS & -25.4 & -8.6 & -6.5 & 19.8 & 11.6 & 10.5 & N: 12636 \\
\hline$K(\mathrm{~dB})$, LOS & 5.3 & 10.0 & 10.3 & 19.8 & 2.6 & 2.1 & N: 2059 \\
\hline$\tau(\mathrm{ns})$ & 35.6 & 151.7 & 151.8 & 268.5 & 40.8 & 33.4 & N: 12636 \\
\hline$S(\mathrm{~ns})$ & 12.8 & 54.9 & 54.0 & 106.7 & 12.4 & 9.8 & N: 12636 \\
\hline
\end{tabular}




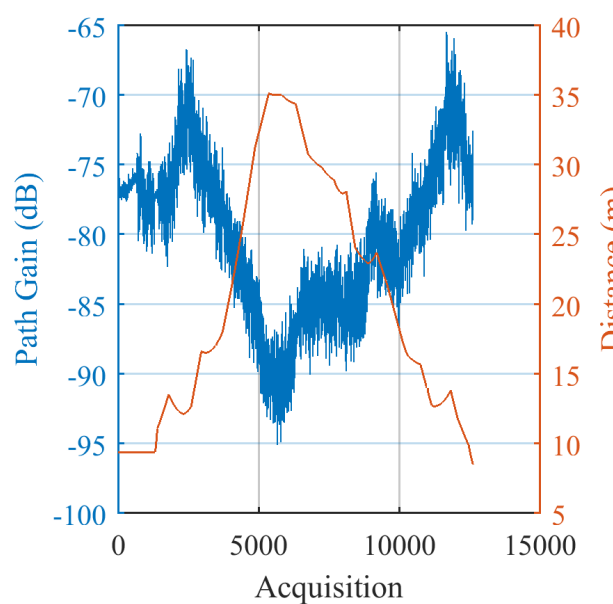

(a) Path Gain versus Acquisition Order

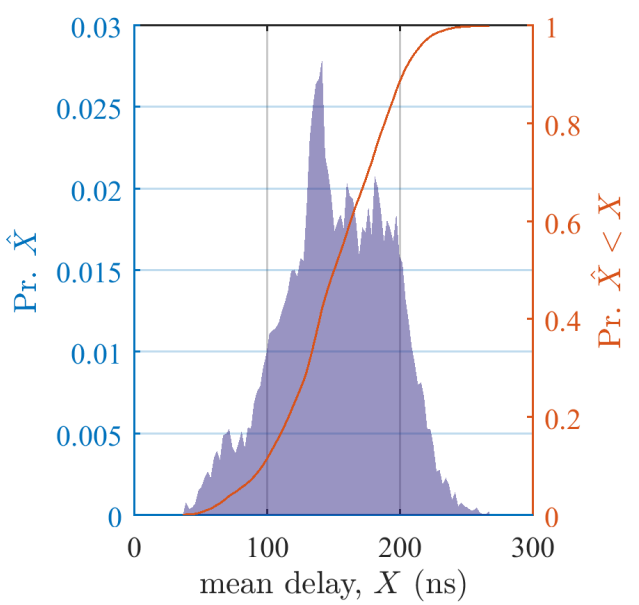

(c) Power Delay

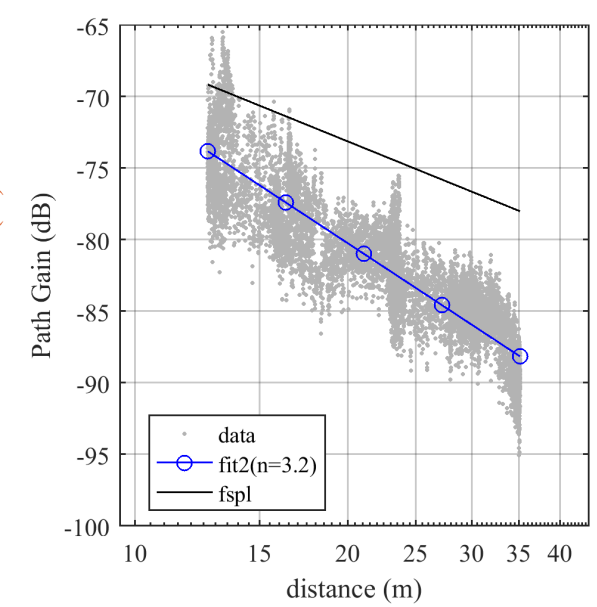

(b) Path Loss versus Distance

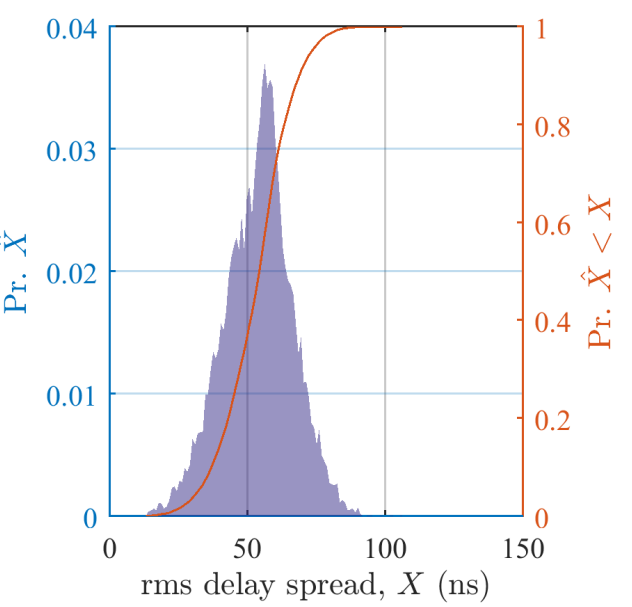

(d) Power Delay Spread

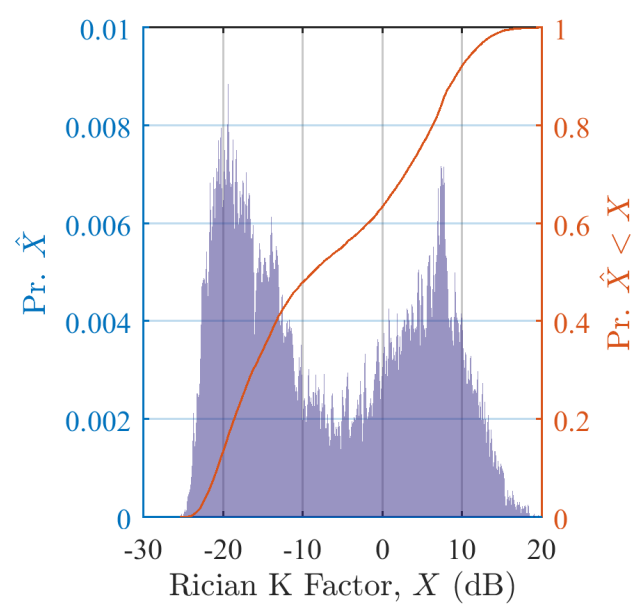

(e) Rician K-factor

Figure A.4.50: Channel characterizations for Tx3 5G 3115Xpol run1 pp channel stats 


\section{A.4.51 Tx3 5G Boiler Vpol run1 pp.mat}

Table A.153: Measurement Parameters

\begin{tabular}{l|c}
\hline Parameter & Value \\
\hline \hline Mat File & Tx3 5G Boiler Vpol run1_pp.mat \\
Frequency (GHz) & 5.400 \\
Location & Omni-directional, V Pol \\
RX Antenna & -3.500 \\
RX Antenna Gain & Omni-directional, V Pol \\
TX Antenna & 3.600 \\
TX Antenna Gain & 3.170 \\
TX Power, Watts & 4.000 \\
PN Oversample Factor & 200.000 \\
Sample rate, MHz &
\end{tabular}

Table A.154: Statistics of Channel Estimates. Outliers are removed using a significance test of 0.95.

\begin{tabular}{l||c|c|c|c||c|c||l}
\hline Estimate & $\min (\cdot)$ & $\operatorname{median}(\cdot)$ & $\operatorname{mean}(\cdot)$ & $\max (\cdot)$ & $\operatorname{std}(\cdot)$ & $\operatorname{mad}(\cdot)$ & outlier info \\
\hline \hline$K(\mathrm{~dB})$, NLOS & -25.1 & -12.8 & -9.3 & 20.4 & 10.5 & 8.7 & N: 10908 \\
\hline$K(\mathrm{~dB})$, LOS & 4.9 & 10.6 & 10.8 & 20.4 & 2.4 & 1.9 & N: 1294 \\
\hline$\tau(\mathrm{ns})$ & 41.0 & 144.3 & 144.2 & 261.1 & 36.8 & 30.6 & N: 10908 \\
\hline$S(\mathrm{~ns})$ & 6.3 & 51.0 & 50.7 & 95.2 & 12.1 & 9.6 & N: 10907 \\
\hline
\end{tabular}

Table A.155: Statistics of Channel Estimates. Outliers are not removed. N indicates the total number of samples in the population. Outliers are provided at 10 and 20 times $\sigma$.

\begin{tabular}{l||c|c|c|c||c|c||l}
\hline Estimate & $\min (\cdot)$ & $\operatorname{median}(\cdot)$ & $\operatorname{mean}(\cdot)$ & $\max (\cdot)$ & $\operatorname{std}(\cdot)$ & $\operatorname{mad}(\cdot)$ & outlier info \\
\hline \hline$K(\mathrm{~dB})$, NLOS & -25.1 & -12.8 & -9.3 & 20.4 & 10.5 & 8.7 & N: 10908 \\
\hline$K(\mathrm{~dB})$, LOS & 4.9 & 10.6 & 10.8 & 20.4 & 2.4 & 1.9 & N: 1294 \\
\hline$\tau(\mathrm{ns})$ & 41.0 & 144.3 & 144.2 & 261.1 & 36.8 & 30.6 & N: 10908 \\
\hline$S(\mathrm{~ns})$ & 6.3 & 51.0 & 50.8 & 106.8 & 12.1 & 9.6 & N: 10908 \\
\hline
\end{tabular}




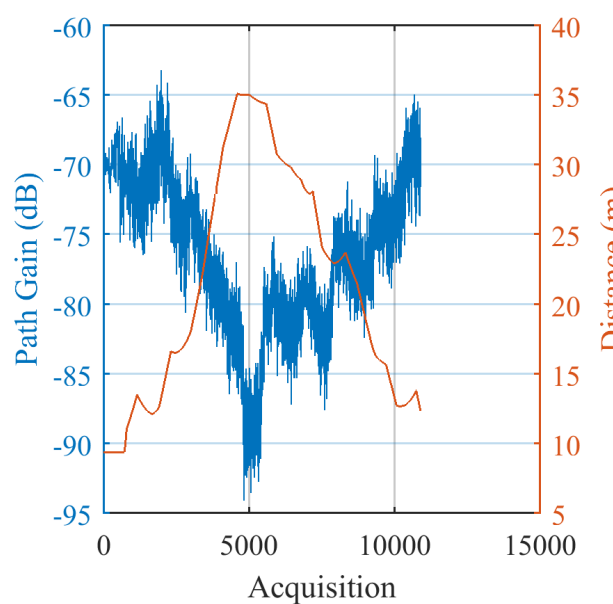

(a) Path Gain versus Acquisition Order

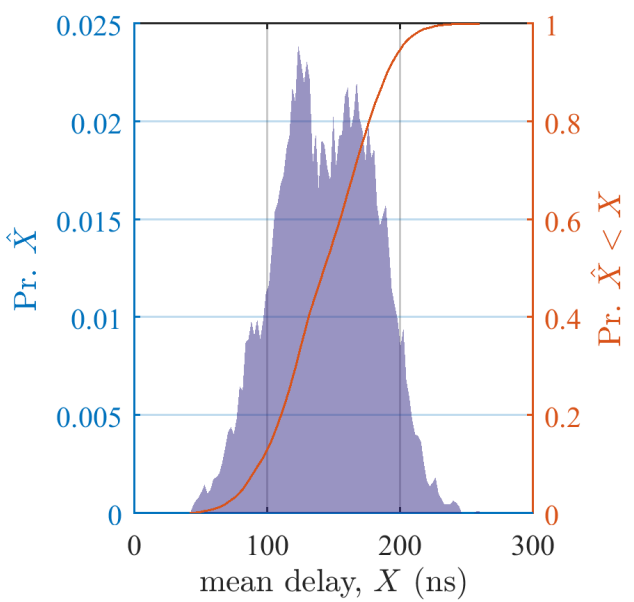

(c) Power Delay

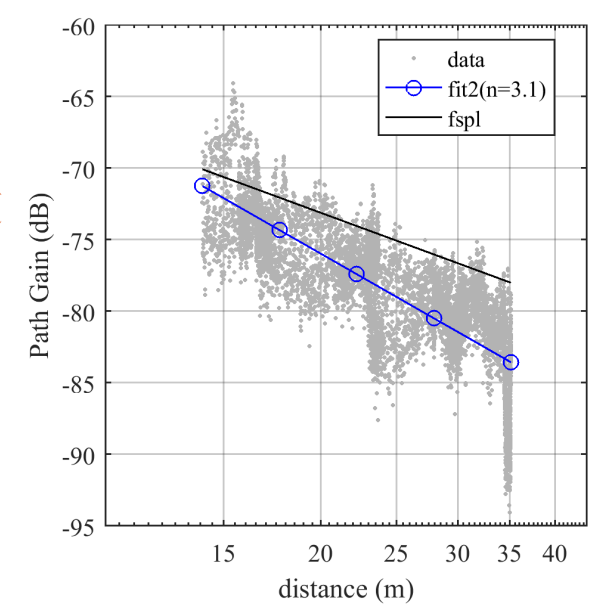

(b) Path Loss versus Distance

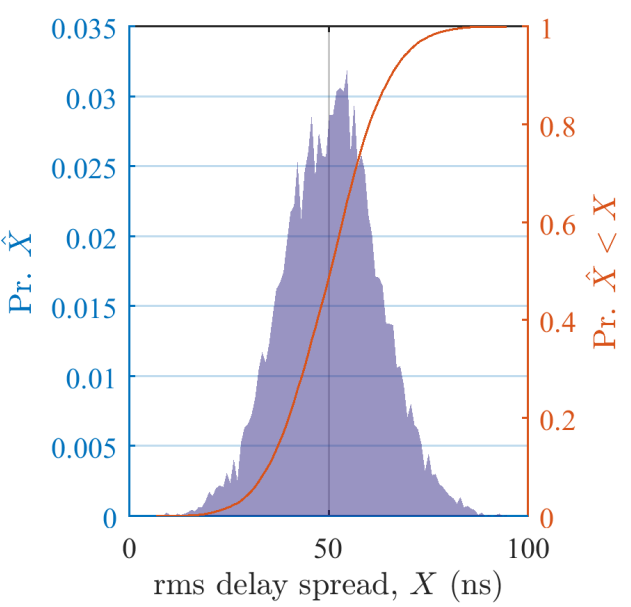

(d) Power Delay Spread

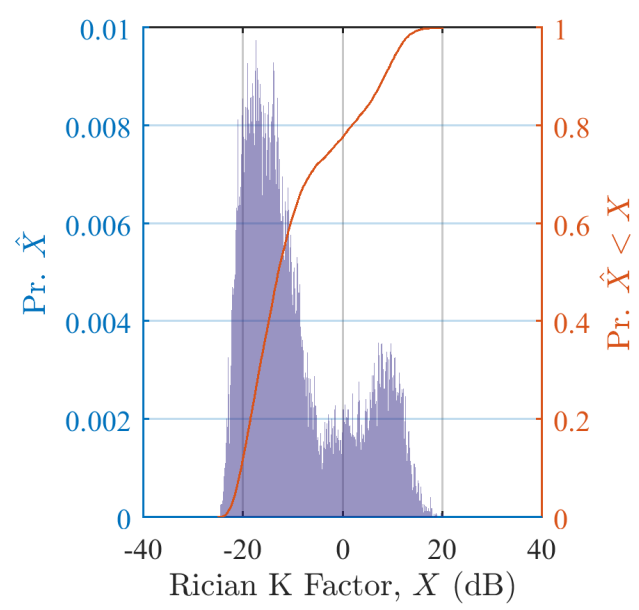

(e) Rician K-factor

Figure A.4.51: Channel characterizations for Tx3 5G Boiler Vpol run1 pp channel stats 


\section{A.4.52 Tx3 5G Boiler Xpol run1 pp.mat}

Table A.156: Measurement Parameters

\begin{tabular}{l|c}
\hline Parameter & Value \\
\hline \hline Mat File & Tx3 5G Boiler Xpol run1_pp.mat \\
Frequency (GHz) & 5.400 \\
Location & Omni-directional, Cross Pol \\
RX Antenna & -3.500 \\
RX Antenna Gain & Omni-directional, X Pol \\
TX Antenna & 3.600 \\
TX Antenna Gain & 3.170 \\
TX Power, Watts & 4.000 \\
PN Oversample Factor & 200.000 \\
Sample rate, MHz &
\end{tabular}

Table A.157: Statistics of Channel Estimates. Outliers are removed using a significance test of 0.95.

\begin{tabular}{l||c|c|c|c||c|c||l}
\hline Estimate & $\min (\cdot)$ & $\operatorname{median}(\cdot)$ & $\operatorname{mean}(\cdot)$ & $\max (\cdot)$ & $\operatorname{std}(\cdot)$ & $\operatorname{mad}(\cdot)$ & outlier info \\
\hline \hline$K(\mathrm{~dB})$, NLOS & -24.9 & -13.6 & -10.6 & 17.0 & 9.7 & 7.9 & N: 12456 \\
\hline$K(\mathrm{~dB})$, LOS & 4.4 & 10.4 & 10.5 & 17.0 & 2.1 & 1.7 & N: 863 \\
\hline$\tau(\mathrm{ns})$ & 45.9 & 148.2 & 148.5 & 270.8 & 38.0 & 31.6 & N: 12456 \\
\hline$S(\mathrm{~ns})$ & 13.5 & 54.8 & 54.9 & 111.8 & 12.5 & 10.0 & N: 12456 \\
\hline
\end{tabular}

Table A.158: Statistics of Channel Estimates. Outliers are not removed. N indicates the total number of samples in the population. Outliers are provided at 10 and 20 times $\sigma$.

\begin{tabular}{l||c|c|c|c||c|c||l}
\hline Estimate & $\min (\cdot)$ & $\operatorname{median}(\cdot)$ & $\operatorname{mean}(\cdot)$ & $\max (\cdot)$ & $\operatorname{std}(\cdot)$ & $\operatorname{mad}(\cdot)$ & outlier info \\
\hline \hline$K(\mathrm{~dB})$, NLOS & -24.9 & -13.6 & -10.6 & 17.0 & 9.7 & 7.9 & N: 12456 \\
\hline$K(\mathrm{~dB})$, LOS & 4.4 & 10.4 & 10.5 & 17.0 & 2.1 & 1.7 & N: 863 \\
\hline$\tau(\mathrm{ns})$ & 45.9 & 148.2 & 148.5 & 270.8 & 38.0 & 31.6 & N: 12456 \\
\hline$S(\mathrm{~ns})$ & 13.5 & 54.8 & 54.9 & 111.8 & 12.5 & 10.0 & N: 12456 \\
\hline
\end{tabular}




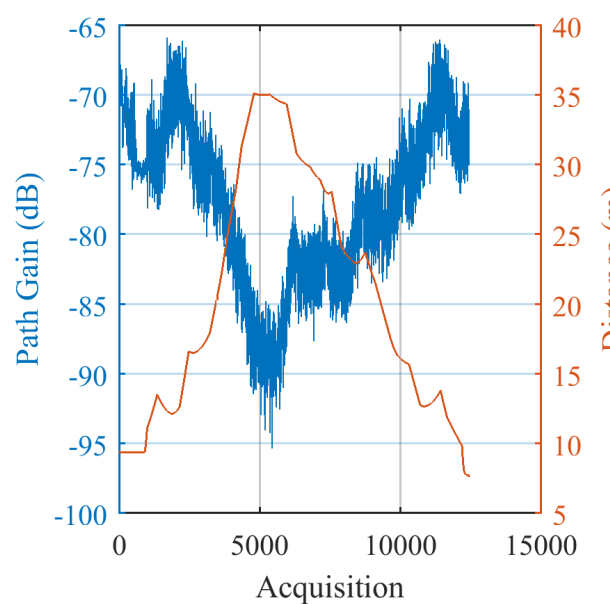

(a) Path Gain versus Acquisition Order

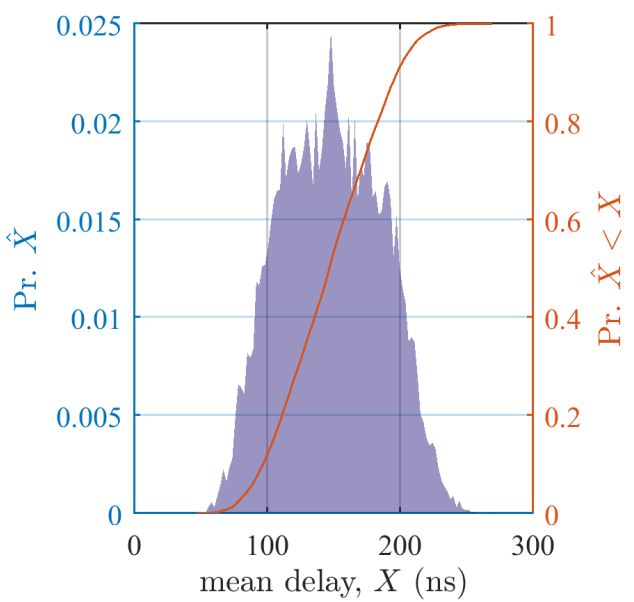

(c) Power Delay

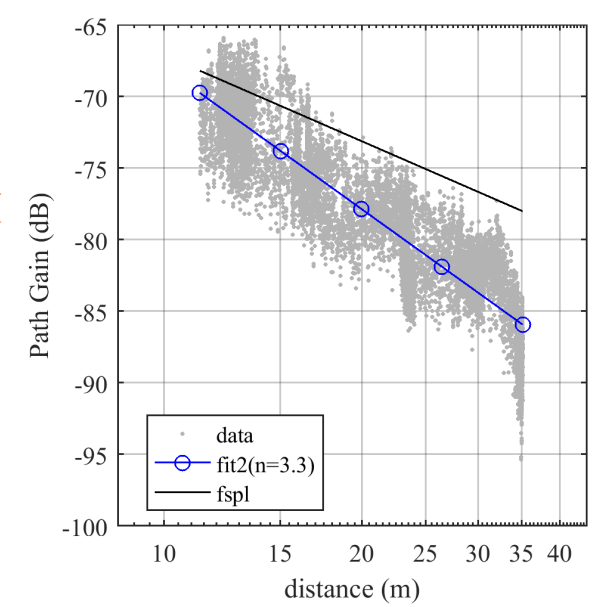

(b) Path Loss versus Distance

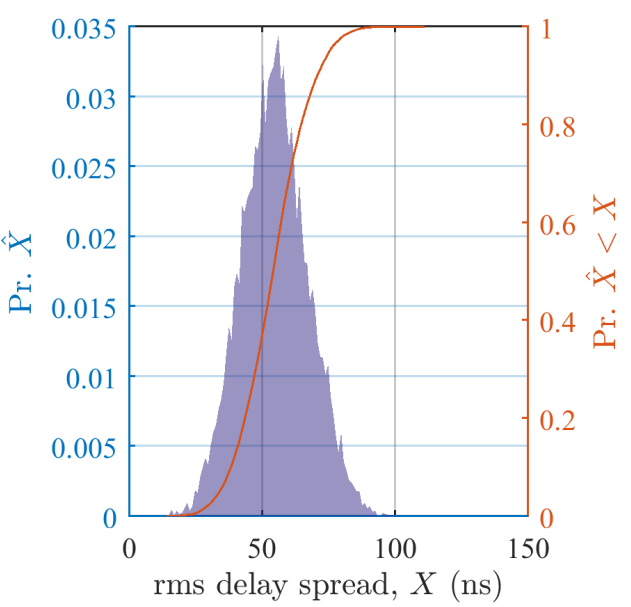

(d) Power Delay Spread

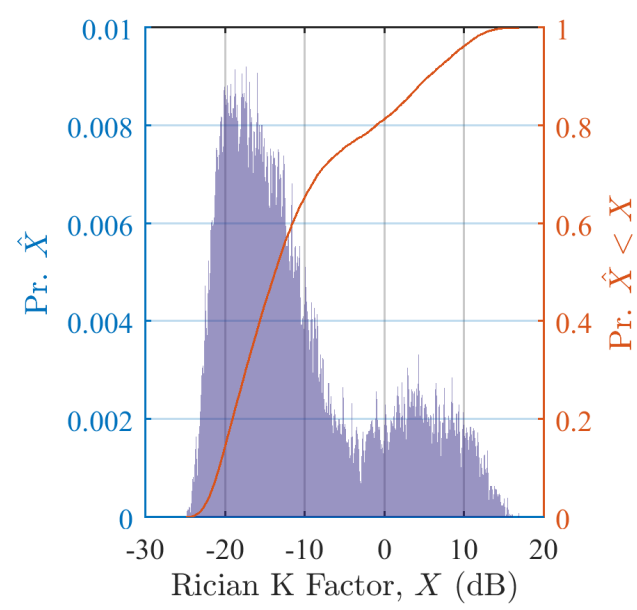

(e) Rician K-factor

Figure A.4.52: Channel characterizations for Tx3 5G Boiler Xpol run1 pp channel stats 


\section{References}

[1] Advanced Manufacturing Partnership 2.0, “ANNEX 1 TRANSFORMATIVE MANUFACTURING TECHNOLOGY: Manufacturing Technology Area 1: Advanced Sensing, Control, and Platforms for Manufacturing," in ACCELERATING U.S. ADVANCED MANUFACTURING, Washington, 2014. [Online]. Available: https://www.manufacturing.gov/news-2/news/reports/

[2] M. Hermann, T. Pentek, and B. Otto, "Design Principles for Industrie 4.0 Scenarios," in 2016 49th Hawaii International Conference on System Sciences (HICSS). IEEE, January 2016, pp. 3928-3937. [Online]. Available: http://ieeexplore.ieee.org/lpdocs/epic03/wrapper.htm?arnumber=7427673

[3] Y. Liu, R. Candell, K. Lee, and N. Moayeri, "A simulation framework for industrial wireless networks and process control systems," in 2016 IEEE World Conference on Factory Communication Systems (WFCS). IEEE, may 2016, pp. 1-11. [Online]. Available: http://ieeexplore.ieee.org/document/7496495/

[4] "Wireless Systems for Industrial Automation: Process Control and Related Applications ISA-100.11a2009 Standard," 2009.

[5] "IEC-62591: Industrial networks - Wireless communication network and communication profiles WirelessHART, 2016.” 2016.

[6] R. Candell, "A Research Framework for Industrial Wireless Deployments," in Proceedings of 2015 ISA Instrumentation Symposium, Huntsville, 2015. [Online]. Available: http://www.nist.gov/el/isd/cs/wsie.cfm, www.isa.org

[7] K. A. Remley, J. Coder, C. A. Grosvenor, R. T. Johnk, G. H. Koepke, J. M. Ladbury, and D. Camell, "NIST-TN-1550 NIST tests of the wireless environment in automobile manufacturing facilities," National Institute of Standards and Technology, Gaithersburg, MD, Tech. Rep., 2008. [Online]. Available: http://nvlpubs.nist.gov/nistpubs/Legacy/TN/nbstechnicalnote1550.pdf

[8] R. Candell, K. A. Remley, and N. Moayeri, "Radio Frequency Measurements for Selected Manufacturing and Industrial Environments," 2016. [Online]. Available: http://doi.org/10.18434/T44S3N

[9] R. Candell, “Computer Code for Industrial Wireless Analysis," Gaithersburg, MD, 2016. [Online]. Available: https://github.com/usnistgov/IndustrialWirelessAnalysis

[10] D. R. Novotny, A. E. Curtin, C. A. Remley, P. B. Papazian, J. T. Quimby, and R. Candell, "A tetherless, absolute-time channel sounder, processing, and results for a complex environment," in Proceedings of the Antenna Measurement Techniques Association, Austin, TX, United States, November 2016.

[11] J. Quimby, D. Novotny, R. Candell, and K. Remley, "NIST Channel Sounder: Wireless Platforms for Smart Manufacturing, NIST Technical Note, in process," National Institute of Standards and Technology, Tech. Rep., 2016.

[12] I. T. Union, "ITU-R 1407-5 Multipath propagation and parameterization of its characteristics," International Telecommunications Union, Tech. Rep., 2013. [Online]. Available: https://www.itu.int/rec/R-REC-P.1407/en

[13] B. Shoelson, U. Schwartz, and V. Makarov, "MATLAB Code deleteoutliers()," 2003. [Online]. Available: https://www.mathworks.com/matlabcentral/fileexchange/3961-deleteoutliers 